\title{
Shared decision-making in oncology
}

Citation for published version (APA):

Ankolekar, A. (2021). Shared decision-making in oncology: challenges and opportunities. [Doctoral Thesis, Maastricht University]. Maastricht University. https://doi.org/10.26481/dis.20211125aa

Document status and date:

Published: 01/01/2021

DOI:

10.26481/dis.20211125aa

Document Version:

Publisher's PDF, also known as Version of record

\section{Please check the document version of this publication:}

- A submitted manuscript is the version of the article upon submission and before peer-review. There can be important differences between the submitted version and the official published version of record.

People interested in the research are advised to contact the author for the final version of the publication, or visit the DOI to the publisher's website.

- The final author version and the galley proof are versions of the publication after peer review.

- The final published version features the final layout of the paper including the volume, issue and page numbers.

Link to publication

\footnotetext{
General rights rights.

- You may freely distribute the URL identifying the publication in the public portal. please follow below link for the End User Agreement:

www.umlib.nl/taverne-license

Take down policy

If you believe that this document breaches copyright please contact us at:

repository@maastrichtuniversity.nl

providing details and we will investigate your claim.
}

Copyright and moral rights for the publications made accessible in the public portal are retained by the authors and/or other copyright owners and it is a condition of accessing publications that users recognise and abide by the legal requirements associated with these

- Users may download and print one copy of any publication from the public portal for the purpose of private study or research.

- You may not further distribute the material or use it for any profit-making activity or commercial gain

If the publication is distributed under the terms of Article $25 \mathrm{fa}$ of the Dutch Copyright Act, indicated by the "Taverne" license above, 
Doctoral thesis

\section{SHARED DECISION-MAKING IN ONCOLOGY: CHALLENGES AND OPPORTUNITIES}

Anshu Ankolekar

2021 
(C) Anshu Ankolekar, Maastricht 2021.

All rights reserved. No part of this publication may be reproduced, stored in a retrieval system or transmitted in any form or by any means, electronic, mechanical, photocopying, recording or otherwise, without prior written permission of the author.

Cover Guus Gijben | proefschrift-aio.nl

Production Guus Gijben | proefschrift-aio.nl

ISBN 978-94-93270-21-3 


\title{
SHARED DECISION-MAKING IN ONCOLOGY: CHALLENGES AND OPPORTUNITIES
}

\author{
Dissertation \\ To obtain the degree of Doctor at Maastricht University, \\ on the authority of the Rector Magnificus, Prof. Dr. R.M. Letschert, \\ in accordance with the decision of the Board of Deans, \\ to be defended in public \\ on 25th November 2021, at 16:00 hours
}

by

Anshu Ankolekar 


\section{Promotor}

Prof. dr. ir. A.L.A.J. Dekker

\section{Co-promotors}

Dr. R.R.R Fijten

Dr. C. Roumen

\section{Assessment Committee}

Prof. dr. G.D.E.M. van der Weijden (chair)

Prof. dr. M.J.G. Jacobs (Tilburg University)

Prof. dr. M.C. Kaptein (Tilburg University)

Dr. D. Raphael

Prof. dr. D.M.R. Townend 


\section{Contents}

1 Introduction 1

1.1 From paternalistic to shared decision-making . . . . . 4

1.2 Putting SDM into practice . . . . . . . . . . . 7

1.3 Challenges in SDM implementation . . . . . . . . . . . 10

1.4 Clinical decision support tools . . . . . . . . . . . 12

1.5 Conclusion . . . . . . . . . . . . . 20

2 The benefits and challenges of using patient decision aids to support shared decision-making in health-care 31

2.1 Introduction . . . . . . . . . . . . . . 33

2.2 Benefits and limitations of PDAs . . . . . . . . . . 35

2.3 The development of PDAs . . . . . . . . . . . 37

2.4 Challenges in the development and implementation of PDAs . . . . . . . . . . . . . . . 44 40

2.5 Future trends . . . . . . . . . . . . . . . . 41

2.6 Conclusion . . . . . . . . . . . . . . 42

3 Development and validation of a patient decision aid for prostate cancer therapy: from paternalistic towards participative shared decision-making 51

3.1 Background . . . . . . . . . . . . . . 53

3.2 Methods . . . . . . . . . . . . . . . . . . 54

3.3 Results . . . . . . . . . . . . . . . . 58

3.4 Discussion . . . . . . . . . . . . . . 67

3.5 Conclusion . . . . . . . . . . . . . 70

4 Implementing shared decision-making in prostate cancer: Insights from primary and secondary care 81

4.1 Introduction . . . . . . . . . . . . . . . 85

4.2 Methods . . . . . . . . . . . . . . . . 86

4.3 Results . . . . . . . . . . . . . . 87 
4.4 Discussion . . . . . . . . . . . . . . . . . . . 92

4.5 Conclusion . . . . . . . . . . . . . . 98

5 Clinician perspectives on clinical decision support systems in lung cancer: Implications for shared decision-making 103

5.1 Introduction . . . . . . . . . . . . . . . . 107

5.2 Methods . . . . . . . . . . . . . . . . . . . . . . . 109

5.3 Results . . . . . . . . . . . . . . . . . . . . 112

5.4 Discussion . . . . . . . . . . . . . . . . . . 120

5.5 Conclusion . . . . . . . . . . . . . . . . . 124

6 Shared decision-making for prophylactic cranial irradiation in extensive-stage small-cell lung cancer: An exploratory study 133

6.1 Introduction . . . . . . . . . . . . . . . . 137

6.2 Methods . . . . . . . . . . . . . . . . . . . . . 138

6.3 Results . . . . . . . . . . . . . . . . . . . 141

6.4 Discussion . . . . . . . . . . . . . . . . . . . . 150

7 Practitioners' views on shared decision-making implementation: A qualitative study 161

7.1 Introduction . . . . . . . . . . . . . . . . . 165

7.2 Methods . . . . . . . . . . . . . . . . . . 167

7.3 Results . . . . . . . . . . . . . . . . . 173

7.4 Discussion . . . . . . . . . . . . . . . . . . 182

7.5 Conclusion . . . . . . . . . . . . . . . . 187

8 General discussion $\quad 199$

8.1 Introduction . . . . . . . . . . . . . . . . . 200

8.2 Summary of main findings . . . . . . . . . . . . . 201

8.3 Strengths and limitations . . . . . . . . . . . . . . 206

8.4 Challenges and opportunities for SDM implementation 207

8.5 Conclusion . . . . . . . . . . . . . . . . 215

$\begin{array}{ll}\text { Summary } & 221\end{array}$

$\begin{array}{ll}\text { Nederlandse samenvatting } & 227\end{array}$ 
Impact statement 233

Acknowledgments

Published work

About the author 



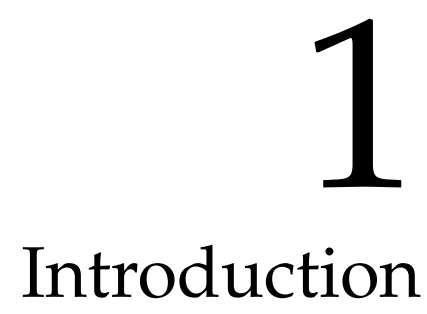


Global healthcare costs are rising and health care providers, insurers and policymakers are grappling with the challenge of improving the quality of healthcare delivered while containing the increasing costs incurred [1]. The calls for a radically new approach focus on shifting healthcare systems from being supply-driven (based on what clinicians and healthcare systems do) to being patient-centered (what patients themselves need and desire), with the ultimate goal to maximize value for patients. Ensuring that patients receive the treatment that is most appropriate and preferable to them can increase patient satisfaction and reduce costs by avoiding over/undertreatment [2]. Patientcenteredness has therefore become a key goal in the journey to achieving better healthcare delivery, and has been described as one of the six core areas that need to be focused on in order to bridge the current health care "quality chasm", the other five areas being safety, effectiveness, timeliness, efficiency and equitability [3].

The relevance of patient-centered care is set to grow in the decades to come, particularly in the field of oncology. The development and treatment of cancerous tumors is not yet fully understood and despite massive strides in cancer therapy it continues to be one of the main causes of death worldwide, leading it to be known as 'the emperor of all maladies' [4]. Three trends are changing the nature of decisionmaking in oncology. First, lifestyle changes and demographic shifts towards aging populations and a rise in the incidence of obesity, particularly in the Western hemisphere, have led to an increase in global cancer incidence and mortality $[5,6]$. This growing disease burden calls for innovative ways to detect, treat, and manage the illness.

Second, new treatments for cancer are being developed at a high rate. Until the late 19th century, surgical removal was the primary treatment for cancerous tumors. The discovery of X-rays in 1895 led to the development of radiotherapy techniques to destroy cancer cells [7]. The use of chemotherapy grew from the 1960s with the discovery of various anti-tumor drugs [8]. More recently, advances in our understanding of the immune system has led to the development of immunotherapy medications [9]. In short, over the last 200 years the possibilities to 
treat cancer have grown multi-fold and so has the life expectancy of people suffering from cancer. Further advances in screening and early detection have added to the number and complexity of decisions to be made, as cancer screening methods can be invasive and come with their own risks $[10,11]$. As a result, cancer treatment trajectories are becoming increasingly decentralized and fragmented. The growing number of therapeutic options, diagnostic testing methods, and number of decisions that need to be made result in patients having to navigate an increasingly complex system [12], and there is often a lack of coordination between the various clinics and care systems a patient might have to go through, particularly for complex diseases [12].

Third, the advent of the "Information Age" has resulted in unprecedented access to data and thus knowledge. New technological developments such as artificial intelligence (AI) and machine learning (ML) can condense large volumes of data into useful information that is easier to understand and are poised to revolutionize healthcare [13]. Increased access to information through the internet and social networks is empowering patients to play a more active role in their treatment decisions. Today, through the internet patients have access to information about their disease and treatment options and are better able to understand their options. While this provides patients with the opportunity to be proactive in their choices, the prevalence of inaccurate information or too much information can make it overwhelming to process and identify what is relevant [14]. Therefore, there is a need to ensure that patients have access to high-quality information that is both accurate and relevant to their individual needs.

These trends have implications for how healthcare is organized and delivered. Healthcare providers must evaluate their current decisionmaking practices, the informational needs of patients, and how trajectories and tools can be designed to facilitate patient participation. 


\subsection{From paternalistic to shared decision-making}

Historically, clinical decisions were made under a paternalistic model, in which the clinician used his/her knowledge and experience to make choices on behalf of the patient. This was a natural consequence of the fact that clinicians were trained in medical matters and the mainstream population had little access to scientific information and therefore little knowledge about diseases, treatments, and consequences. Although clinicians' choices were meant to be in the patient's best interest, the patient's wishes were not formally included in the decisionmaking process [15]. Even with the rise in the notion of patient autonomy in the 1960s, alongside similar movements regarding human rights and autonomy in general $[16,17]$ and the introduction of informed consent, an information asymmetry remained between clinicians and patients, and decisions were still largely paternalistic until the 1970s, based mainly on clinical guidelines [18].

Growing evidence over decades of randomized clinical trials has shown that many treatment modalities do not have beneficial effects in all patients [19]. Due to patients' individual genetic profiles, lifestyle factors, and behaviors, one treatment may not affect all patients in the same way and indeed may show only marginal benefits or none at all for certain patients [20]. In addition, a number of disease areas are preference-sensitive, i.e. the evidence indicates that one treatment is not clinically superior to another and therefore the treatment choice depends on how the individual patient weighs the pros and cons of each [21].

The process of patients and clinicians working together to evaluate treatment pros and cons taking into account the patient's own values and preferences has become known as shared decision-making (SDM). SDM is founded on the premise that while clinicians are experts in the clinical domain, patients are experts on their own lives, and to the extent that cancer treatment decisions impact their daily lives, patients ought to be partners in the decision-making process [22]. With the accompanying rise in patient autonomy and patients' preference for par- 
ticipation in their healthcare decisions, the nature of clinical decisionmaking is shifting from paternalistic to participatory [23].

Paternalistic decision-making and SDM differ on four dimensions [24]:

1. the level of involvement of the patient in the decision-making process

2. the direction of information exchange between clinician and patient

3. the type of information that influences the treatment decision

4. the decision-maker(s)

These differences are summarized in Figure 1.1. Under a traditional paternalistic model, information exchange is largely one-way: from the clinician to the patient. The clinician provides medical information such as all available treatment options and their benefits and risks, and the amount of information provided might only be the minimum required. In contrast, under the SDM model, information flows both ways between the clinician and the patient: the clinician provides all relevant medical information and the patient contributes information about their values, lifestyle, and own knowledge about the disease and its treatment. In this manner all relevant information is shared between both parties and decisions can be made from a common ground. The deliberation stage of decision-making involves expressing treatment preferences and weighing the different treatment options. Under the paternalistic model, the clinician considers all treatment options and chooses one, at most in consultation with other clinicians. The deliberation stage in the SDM model is more interactive in nature, with patients offering their own perspective on the most desired course of action. In the final stage the treatment is chosen; under SDM this choice is made by both patients and clinicians, while in a paternalistic approach the clinician would simply make the decision alone based on their own knowledge and experience. 


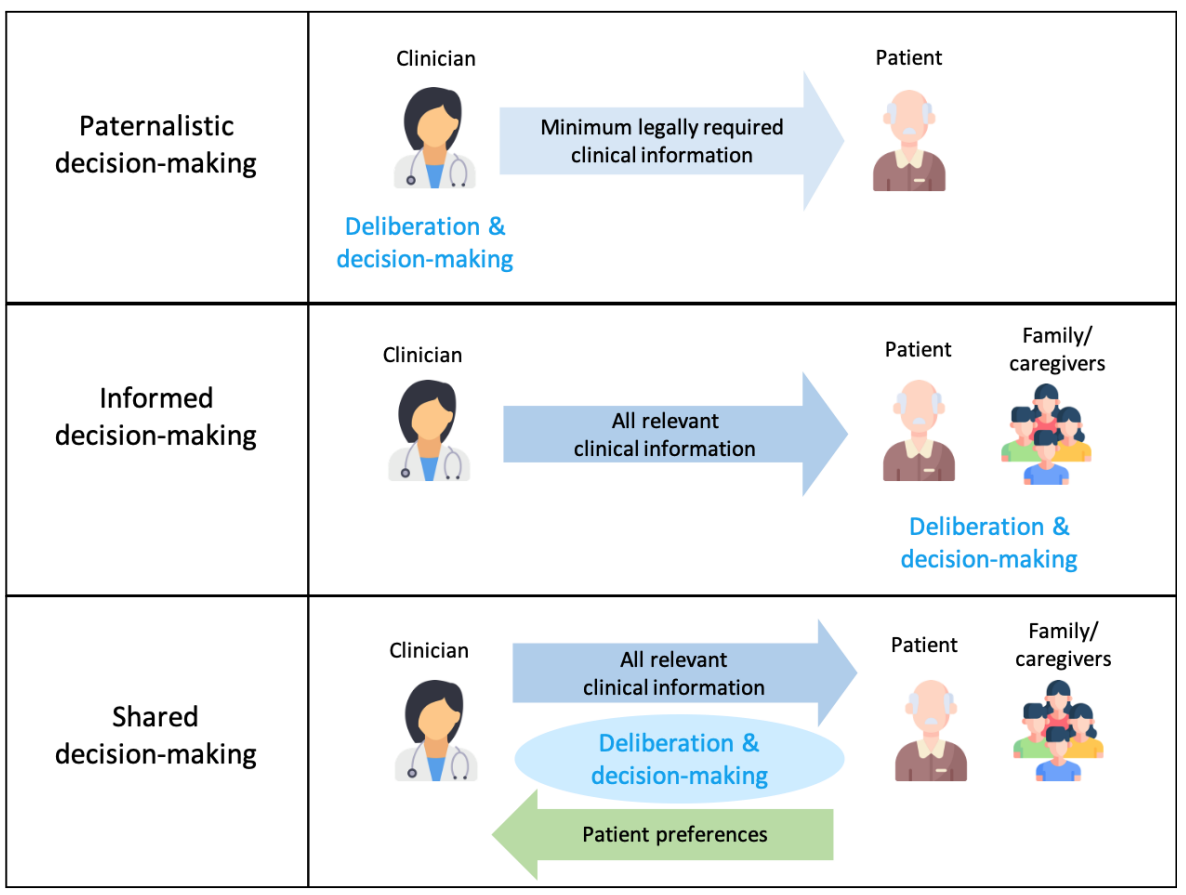

Figure 1.1: Models of clinical decision-making (adapted from Charles et al., 1999)

SDM is sometimes conflated with informed decision-making and the concept of informed consent, and although there is some overlap between the three principles, they are distinct from one another. Under the informed decision-making model, information exchange remains in one direction (from clinician to patient) and consists of all relevant clinical information, such as harms and benefits of different treatment options, rather than the minimum required as under the paternalistic model. Patient preferences do not form a component of the information used to make a treatment choice, as they do under the SDM model. Informed consent refers to the legal and ethical process of providing a patient with the information about their disease, treatment, risks, and benefits necessary to consent to the treatment [25]. It does not, how- 
ever, outline the patient's role in the process to reach that treatment decision. SDM thus goes beyond informed consent by emphasizing the relational aspect between clinicians and patients and the communication process to arrive at clinically sound decisions based on patient choices [26].

There is growing evidence that patients benefit greatly from taking a more proactive approach in their healthcare decisions. For instance, patients who are better informed about their treatment options are more likely to adhere to their treatments [27]. Furthermore, greater collaboration between patients and their clinicians can benefit the healthcare system as a whole in the form of a reduction in costs and unnecessary/unwanted medical procedures [2]. At the same time, it is important to be aware that there are various degrees of patient participation and that patients vary in their desired level of participation. While around $50 \%$ of patients prefer a collaborative role, in which the decision-making process is shared equally between patient and clinician, the remaining 50\% are roughly evenly split between preferring an active (patient-driven) or passive (clinician-driven) process [28]. Furthermore, patients are not always aware of what SDM entails or ready to participate, therefore clinicians play a critical role in inviting patients into the SDM process [29].

\subsection{Putting SDM into practice}

To assist clinicians in putting the elements of SDM into practice, it has been conceptualized as a three-stage process consisting of a choice talk, an option talk, and a decision talk, as shown in Figure 1.2 [30]. In the choice talk, the clinician explicitly verbalizes to the patient that several treatment options are available and a treatment choice needs to be made together. The option talk involves informing the patient about these different treatment options, the pros and cons of each, and gauging the patient's level of understanding of the options by asking questions. Finally, in the decision talk the clinician elicits what matters to the patient and supports the patient in deriving their preference. If 
the patient is ready to make a decision, a treatment in line with the patient's preference is chosen. If not, the clinician and patient opt to defer the decision and give the patient time to consider the choices further and with others, such as family or caregivers. By moving through the three stages, the patient's initial preferences that were based on basic information are converted into informed preferences that result from a joint evaluation of the clinical evidence and its implications in terms of the patient's personal circumstances.
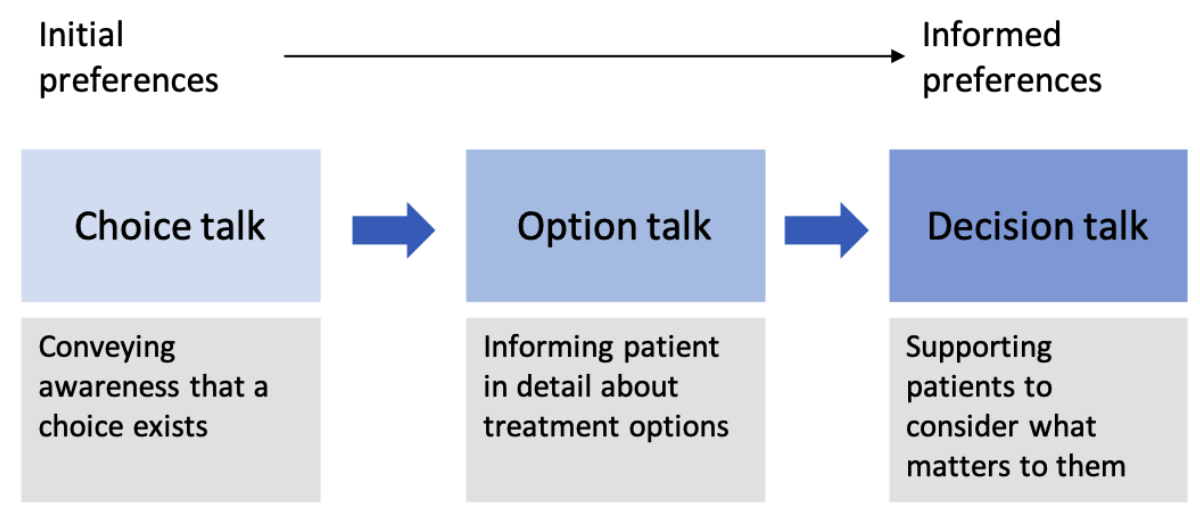

Figure 1.2: The traditional three-talk SDM model (adapted from Elwyn et al., 2012)

Other conceptualizations include the SHARE approach and the SDM 3 Circle Model [31,32]. The SHARE approach helps clinicians invite patients into the SDM process through five steps summarized by the acronym 'SHARE': Seeking the patient's participation, Helping the patient compare the treatment options, Assessing the patient's values and preferences, Reaching a decision together, and Evaluating the decision. Clinicians learn effective communication through role-plays, case studies, and patient-centered outcomes research so that SDM does not remain a static five-step process but a dynamic, engaging conversation. This approach has been implemented successfully in several clinics in the United States [33,34]. 
The SDM 3 Circle Model recognizes the influence of additional factors, such as the clinic environment, the perspectives of the patient's caregivers/family members, and the medical context (e.g. the tests, medications, and other procedures in the treatment trajectory). It depicts the SDM process as a Venn diagram of circles representing these factors (Figure 1.3). SDM is achieved when these three aspects are aligned in a dynamic process of gathering and sharing information (both clinical and patient-related), deliberating on the pros and cons of the options, and implementing and evaluating the shared decision. This model is relatively new and yet to be applied in a widespread manner, however it is one of the first to go beyond the patient-clinician interaction and incorporate external factors in the SDM process.

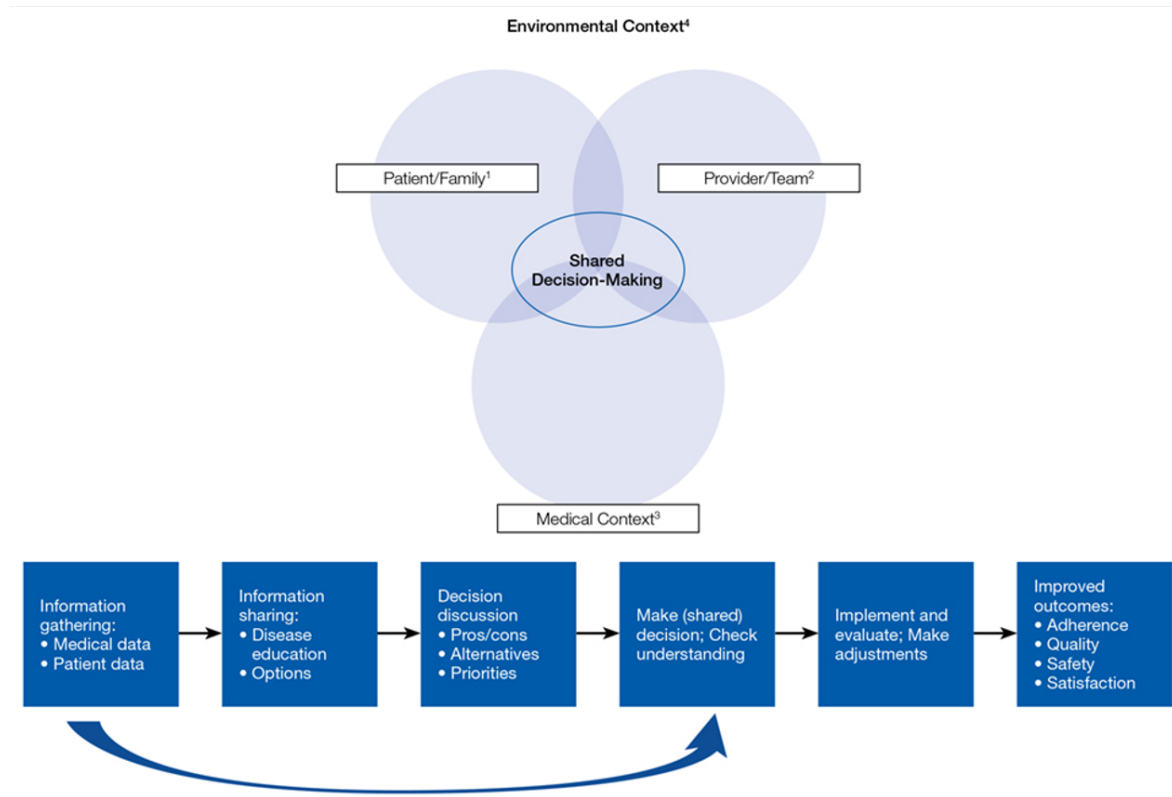

Figure 1.3: The 3 Circle SDM model (adapted from Rennke et al., 2018)

As the previous three examples have shown, SDM at its core seeks to support the patient in uncovering the harms and benefits of various treatment options and the implications of these harms and benefits on 
the patient's individual situation. The simplest iteration of the SDM model is therefore the ' 3 Good Questions' model developed in Australia and popularized in the United Kingdom and the Netherlands [35,36]: "What are my options?"; "What are the possible benefits and harms of those options?"; "How likely are the benefits and harms of each option to occur?". The afore-mentioned studies have found that asking these three basic questions improves patient-clinician communication, the quality of information provided, and certain SDM metrics (OPTION scores and CollaboRATE).

\subsection{Challenges in SDM implementation}

However, implementing SDM into routine clinical practice has several challenges, the first one being the training of care professionals. The components of SDM vary according to different clinical contexts and there is no single agreed-upon model for SDM, e.g. SDM for making treatment decisions in oncology is slightly different from SDM for screening decisions or nursing [37]. As a result, there remains a lack of large-scale evaluations of different training programmes worldwide to determine which components of the training process are most effective [38]. Training initiatives designed to teach SDM skills to healthcare providers must take into account the components of SDM relevant to the clinical situation.

The second challenge to SDM implementation is practical/logistic; involving patients in treatment decisions requires an adjustment to clinical workflows, such as additional time to inform patients and elicit their preferences, as well as developing and disseminating additional patient information materials. Organizational barriers to SDM have been less extensively studied but evidence suggests that organizational leadership, culture, priorities and resources are crucial factors that influence SDM implementation [39]. A recurring theme across these dimensions, and the most frequently cited barrier to SDM according to clinicians, is time constraints due to busy work schedules [40]. Randomized controlled trials find inconclusive evidence that SDM consul- 
tations are longer than usual care; two studies found that SDM took longer, one found SDM consultations shorter, and six concluded that the differences were statistically insignificant [41]. However, the perception persists that SDM would require too much time [42], and as a result clinician resistance is a major challenge to SDM implementation.

This brings us to the third and perhaps most challenging barrier to overcome: behavioral change. SDM requires clinicians to shift from the traditional decision-making model that has been prevalent for decades to one that involves the patient as an equal partner in the process. Aside from resistance due to time constraints, clinicians most often cite a lack of applicability of SDM because of the patient's characteristics (age, literacy level, cultural factors), and due to the clinical situation [40]. Secondary barriers include self-reported unfamiliarity with the principles of SDM, reluctance to ask patients about their desired role in making treatment decisions (due to pre-conceived ideas that the patient would not be interested in engaging in SDM), a perceived lack of self-efficacy, and the belief that certain patient preferences cannot be reconciled within an SDM framework.

In other cases, clinicians are enthusiastic about SDM and believe they are practicing it but evidence from observation-based studies indicates that SDM is not being applied to a significant extent [43]. This may be due to a lack of clarity on the part of the health care professionals about the actual principles of SDM and how to apply them in practice. As mentioned previously, another possible reason is that SDM is often confused with the concept of informed consent. The result is that even clinicians who are motivated to involve their patients fail to actualize the benefits of SDM due to improper application.

SDM also requires a change in behavioral change on the patients' side, as they must now take an active part in the decision-making process. Patient-reported barriers typically involve the organization of the healthcare system [44]. Lack of consultation time and busy doctors' schedules are the most common factors that discourage patients from 
seeking more engagement with their doctors [45-47]. Some factors beyond the patient's control, such as dementia, can diminish the patient's ability to engage in SDM [48]. Individual factors such as age, gender, ethnicity, and personal beliefs also affect patients' willingness to take a more active role in their treatment. There is a common perception that older patients do not wish to be involved in SDM, however there is evidence that clinicians can alleviate this barrier by spending additional time eliciting patients' goals, expectations, and knowledge of their options [49-51]. Summarizing, the evidence suggests that if patients do not participate in SDM it is more likely due to factors beyond their control rather than due to a lack of enthusiasm.

Clinician motivation is therefore a key facilitator to SDM, along with perceived positive impact on the clinical process and on patient outcomes [40]. In other words, clinicians are more likely to engage in and encourage SDM with patients when they are convinced that the process is more efficient and patient satisfaction is higher. Managing the motivations and perceptions of health care professionals is therefore a key aspect for enhancing SDM, alongside providing decision support tools that help inform patients so that they are able to deliberate over their options and make an informed decision.

\subsection{Clinical decision support tools}

For patients and clinicians to make decisions together, it is necessary that both have access to information and high-quality evidence about the different treatment options. Clinical decision support tools summarize and present relevant information about a specific condition so that participants in the decision-making process can understand and discuss the most relevant information related to a particular decision. These include patient decision aids (PDAs) for patients and clinical decision support systems (CDSSs) for clinicians. Such tools are not prerequisites for SDM but can provide a succinct overview of clinical information that allows participants to deliberate more effectively. 


\subsubsection{Decision support for patients}

Patient decision aids are information tools, in a printed or digital format, that provide an overview about the disease and its treatment options, the harms and benefits of the different treatments, and optionally some methods for the patient to weigh these different options in terms of their pros and cons. Digital PDAs typically consist of an interactive set of questions designed to understand the risk profile of patients and then present treatment options that are in line with their preferences regarding risk attitudes and lifestyle. Figure 1.4 presents an example of a digital PDA for colorectal cancer screening tests. The aim of such tools is to establish a common ground between the patient and the clinician so that the patient understands their treatment options better and the clinician understands the patient's preferences so that both can collaborate to choose the course of action that is associated with the best health outcome for the patient. Chapter 2 provides an overview of PDAs and the process of developing them.

Such PDAs are typically meant to be used by the patient in their own time outside the consultation, for instance at home. Other forms of decision aids, such as option grids and decision cards, are designed to be used by patients and clinicians together in the consultation. An option grid is a single-page overview of the benefits and harms of various treatments for a disease based on frequently asked patient questions (Figure 1.5). Decision cards are a collection of pocket-sized cards conveying information about different treatment options, with an emphasis on simple graphics and information rather than in-depth explanations (Figure 1.6). These in-consultation decision aids are meant to stimulate dialogue between patients and clinicians [52].

Cochrane Level 1 evidence from 55 randomized trials indicates that PDAs are associated with improved patient knowledge about diseases and their treatment options, and lower decisional conflict [55]. Patients who use PDAs are better informed about their condition, more likely to adhere to their treatments, and typically experience less anxiety, fatigue and depression, and in some cases faster recovery [56]. 


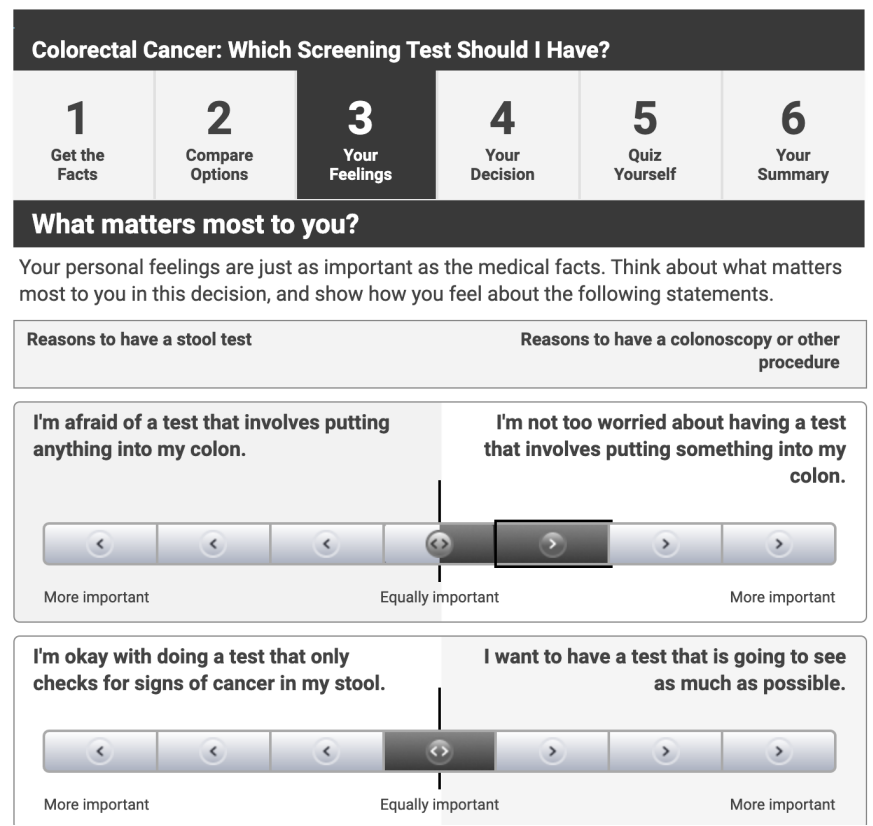

Figure 1.4: Example of a web-based PDA for colorectal cancer (Source: Healthwise - https://www.healthwise.net/ohridecisionaid/)

The format of the PDA (digital or print) does not appear to affect the level of patient knowledge, patient participation in decision-making, or decisional conflict, however one study comparing the two formats found that patients tend to use the print version of the PDA more than the web-based version, and patients' preferences between the two formats were influenced by age, race, and education level [57]. The study also found that the values clarification component of the PDA was more often completed in the web-based PDA than in the print PDA where it was provided as a worksheet. Developing a PDA therefore requires a thorough understanding of the target patient group's needs and preferences. This process is explored in Chapter 3 which describes the development of a PDA for prostate cancer patients based on a usercentered design. 
Early stage breast cancer: What's right for me?

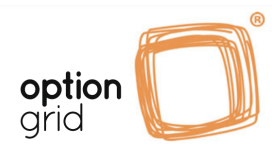
Use this Option Grid to help you and your health care professional decide how best to treat early stage breast cancer (stages I to IIIA).

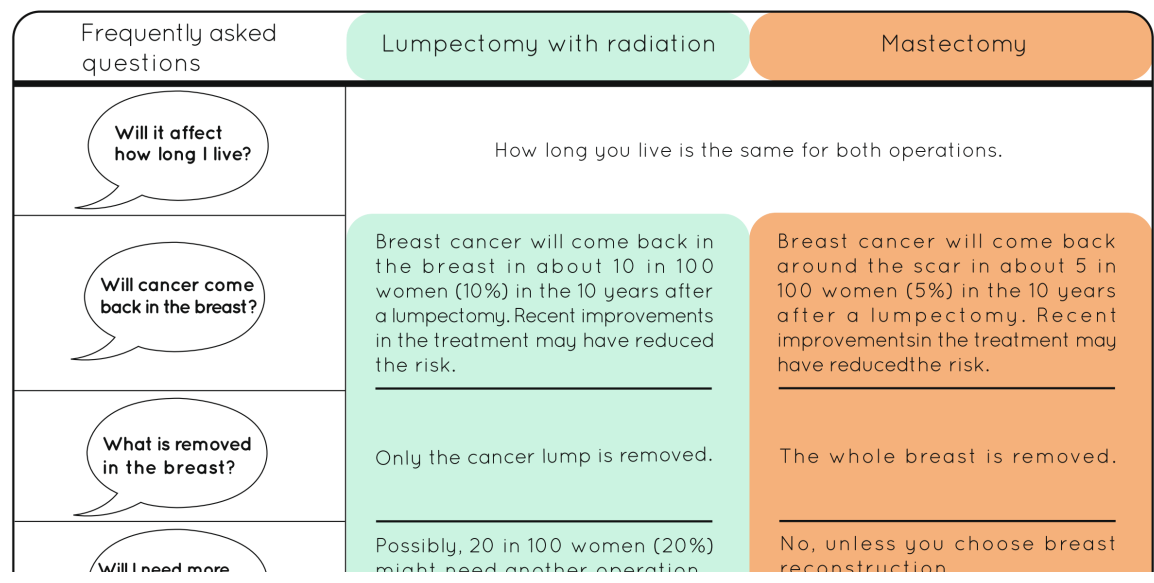

Figure 1.5: Example of an option grid for early-stage breast cancer [53]

As with any new innovation, introducing a PDA into clinical practice is challenging. The barriers to implementing PDAs in clinical practice range from PDA-specific factors (quality, usability, and maintenance) to user-related factors such as ensuring that clinicians have the required knowledge and skills in using the PDA in an SDM process and that patients have sufficient health literacy to process the complex clinical information they are faced with in making a decision. For instance, health literacy is strongly linked to access to and experience with using the internet, which in turn is influenced by demographic and socio-economic factors such as age, education level, and income [58]. Successful PDA implementation must confront this digital divide. Finally, structural/organizational factors can hinder PDA implementation if existing clinical workflows, time schedules, and the roles of various personnel (such as clinicians, nurses, hospital managers, and administrators) are not aligned [59]. Implementing PDAs therefore re- 


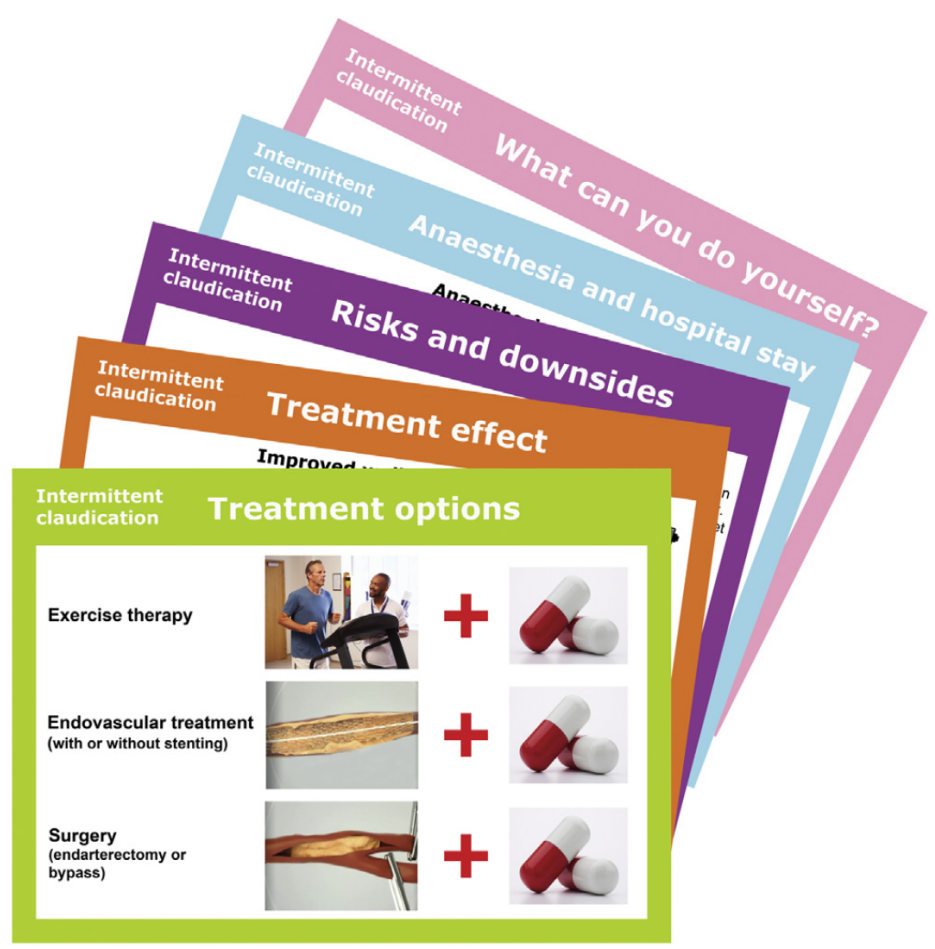

Figure 1.6: Example of a set of decision cards for carotid artery disease [54]

quires thorough consideration of not only user experience and clinical workflows, but also the wider organizational culture and healthcare infrastructures. Chapter 4 explores these issues in the context of the digital prostate cancer PDA referred to in Chapter 3. We supplement our exploration of this topic in Chapter 7 which presents a case study of the implementation of SDM supported by print PDAs in Vejle Hospital, a hospital specialized in cancer treatment in the region of Southern Denmark.

PDAs offer several advantages: patients can read and absorb clinical information at their own pace, discuss the information with their family and caregivers, and search for supplementary information to fill in 
gaps in their knowledge. They may also take the time to fill in value clarification questions to discover what matters to them regarding the treatment options, and can then prepare for the deliberation process in the consultation. One disadvantage is that it is not always clear to what extent patients use PDAs outside the consultation and whether it has sufficiently prepared them to take part in SDM [60]. Table 1.1 summarizes the various forms of patient decision support tools we have described thus far.

Table 1.1: A summary of the three main types of types of patient decision support tools

\begin{tabular}{llll}
\hline Type of tool & Description & Format & Application \\
\hline $\begin{array}{l}\text { Patient decision aid } \\
\text { (PDA) }\end{array}$ & $\begin{array}{l}\text { A tool that provides information about a disease, the } \\
\text { different treatment options available, and the } \\
\text { benefits and harms of each option. }\end{array}$ & $\begin{array}{l}\text { Digital or } \\
\text { print }\end{array}$ & $\begin{array}{l}\text { Usually given to patient } \\
\text { for use outside the } \\
\text { consultation, but can } \\
\text { also be used in- } \\
\text { consultation }\end{array}$ \\
Option grid & $\begin{array}{l}\text { A single-page overview of the benefits and harms of } \\
\text { various treatments for a disease based on } \\
\text { frequently asked patient questions. }\end{array}$ & $\begin{array}{l}\text { Digital or } \\
\text { print }\end{array}$ & In-consultation \\
& $\begin{array}{l}\text { A collection of pocket-sized cards conveying } \\
\text { information about different treatment options, with } \\
\text { an emphasis on simple graphics to facilitate } \\
\text { understanding and comparison. }\end{array}$ & Print & In-consultation \\
\hline
\end{tabular}

\subsubsection{Decision support for clinicians}

Clinical decision support systems (CDSSs) are defined as "electronic systems designed to aid directly in clinical decision making, in which characteristics of individual patients are used to generate patient-specific assessments or recommendations that are then presented to clinicians for consideration" [61]. These can range from classic alerts and reminder systems that prompt clinicians to take certain actions based on pre-specified criteria to information systems linked to patient electronic health records (EHRs) that allow clinicians to generate and view specific treatment recommendations based on the predicted outcomes [62]. An example of a predictive CDSS for a 
fictitious patient is displayed in Figure 1.7.

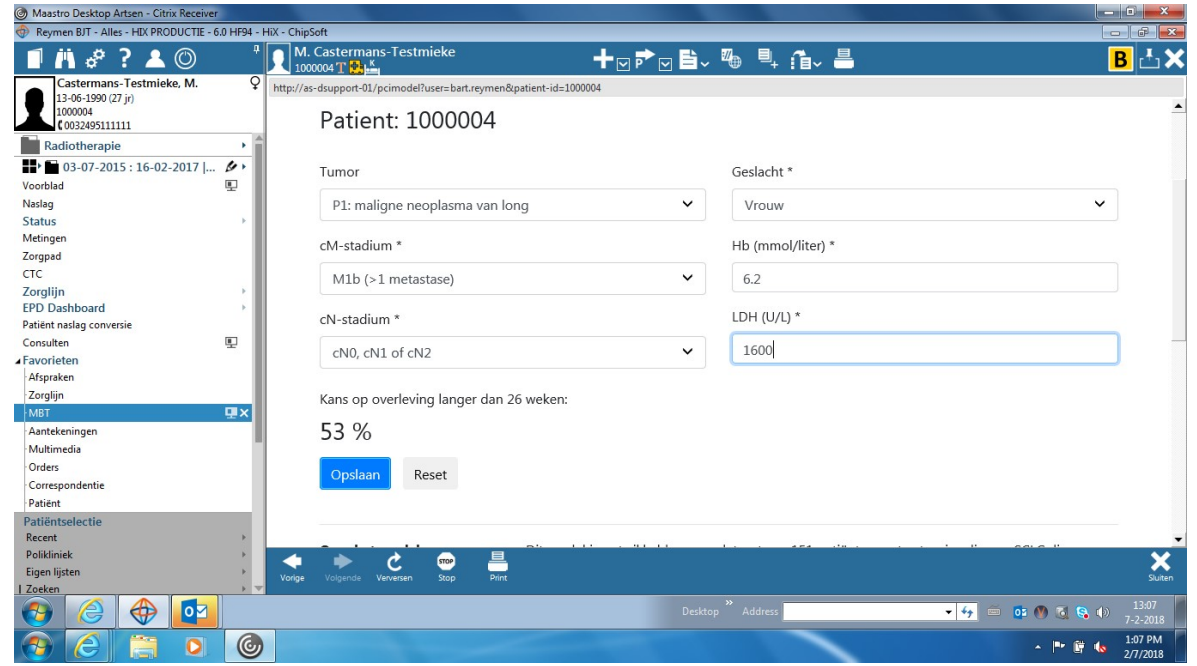

Figure 1.7: Example of a (Dutch) CDSS for predicting survival in extensivestage small-cell lung cancer patients based on the patient's gender, tumor characteristics, and serum markers. This CDSS has been built into the hospital's EHR so that the model can be auto-populated by accessing data directly from the patient's dossier. The data in this image is for a fictitious test-patient (Source: MAASTRO Clinic)

Using a CDSS to predict which patients will likely respond best to certain therapies can help tailor treatments to individuals and improve clinical outcomes, quality of life and avoid over/under-treatment (which can be costly in the short- and long-term). For instance, a systematic review of ten implemented CDSSs for treatment choices in oncology shows that five had a statistically significant positive effect on process outcomes such as treatment adherence, and four were associated with an improvement in patient outcomes [63]. However, these possibilities are slow to be realized in clinical practice.

The lack of widespread use of CDSSs is attributed to lack of familiarity and trust from clinicians, time pressures, and poor integration into the clinician workflow [64], and although these are important consid- 
erations, fixing them is not enough to guarantee uptake. CDSSs must address clinical use cases in which they (feel they) need support in order for clinicians to perceive them as useful additions to their workflow [65]. In other words, different barriers exist at different implementation stages; initially, clinicians may be resistant because of ideological disagreements with CDSSs in their practice or because they feel that decision making in a given situation does not need to be supported, e.g. because there is a clear preferential decision. Once they are open to the value of a CDSS the issues become more practical (usability, integration into workflow) [66]. Those who are positive towards CDSSs may still recognize the risks associated with them, such as potential distress for patients regarding predictions as well as prognostic overconfidence [67]. These findings warrant an exploration of the decision-making process among clinicians, what role CDSSs may play in decision-making and what the implications are for SDM. These topics are explored in Chapters $\mathbf{5}$ and $\mathbf{6}$.

\subsubsection{Data-driven shared decision-making}

The rise in the availability of large sets of patient data, such as genetic profiles and imaging, is prompting clinicians to shift from making decisions purely based on clinical characteristics to considering the patient's individual characteristics, aside from their values and preferences [68]. This added complexity and the imperative to include the patient perspective in consultations is paving the way for a third form of decision support tool that combines PDA and CDSS outputs into a common interface for patients and clinicians so that individualized treatment options can be discussed [69]. Existing examples include simple dashboards giving patients access to their medical health record and possibly communicating with their clinician [70]. This form of decision support is still in its infancy, primarily due interoperability challenges, i.e. ensuring that the different data management systems that store patient data can communicate with each other and exchange relevant data without compromising patient safety and privacy [71]. 
Data-driven SDM implies a greater involvement of patients in processing prognostic data such as risk estimates of survival and/or sideeffects. This brings with it a fresh set of challenges in risk communication and interpretation. Prior findings have established that patients, and even clinicians, face difficulties in accurately interpreting risk estimates [72,73]. The way risk information is presented can also influence decision-making, as demonstrated by the framing effect, in which patients make different choices depending on whether the same information is presented in positive or negative terms [74,75]. While these studies indicate certain measures can help to 'de-bias' decision-makers to an extent, other biases are less simple to correct. For instance, due to the knowledge asymmetry and perceived authority of clinicians, often characterized by the phrase "Doctor knows best," patients may also tend to defer to the opinion of their clinician and agree to treatments that are not aligned with their preferences [76]. Therefore, alongside the technological improvements in these tools, the influence of behavioral/psychological factors must also be kept in mind.

\subsection{Conclusion}

Newly diagnosed cancer patients face an increasing number of treatment options that impact their lives in different ways. In certain conditions, there are multiple treatment options with no clinically superior treatment and therefore the choice depends on how the patient weighs the harms and benefits of each treatment option in relation to their own individual circumstances and life situation. Initiatives such as SDM and PDAs encourage patient autonomy and engagement in treatment decisions and have shown promising effects on patient satisfaction and clinical outcomes. However, implementing any new initiative is challenging, and SDM and PDAs are no exception. Successfully bringing SDM and PDAs into routine practice requires an understanding of the barriers at various levels (PDA-specific, patient/clinician, and organizational) so that effective implementation strategies can be defined and tested. 


\subsubsection{Aim and research questions}

The aim of this thesis is to present the challenges of implementing SDM in the clinical pathway in different contexts, and possible solutions. The following research questions are addressed:

1. How to develop a PDA that fulfills user requirements and clinical standards?

2. What do clinicians perceive as barriers and facilitators to implementing SDM in prostate cancer/preference-sensitive conditions?

3. How do these barriers and facilitators manifest in less preference-sensitive conditions, such as lung cancer?

4. How can SDM and PDA delivery be optimized to account for the barriers and facilitators?

\subsubsection{Thesis outline}

This thesis begins with a review of PDAs (Chapter 2). We present an overview of the current literature on the potential of PDAs and the challenges in their implementation.

Chapter 3 describes the process of developing a PDA for prostate cancer patients based on a user-centered design.

Chapter 4 explores factors that affect SDM and PDA implementation from the perspective of clinicians across the prostate cancer treatment trajectory. We present insights gained from interviewing general practitioners (GPs), urologists, radiation oncologists, and oncology nurses, and present a care pathway that may support prostate cancer patients in participating in the treatment decision.

The previous chapters explore SDM in a preference-sensitive condition (prostate cancer). In Chapter 5, we consider the opportunities and challenges for SDM in lung cancer, a disease area characterized 
by poor prognosis and rapid progression. This disease typically does not afford patients the opportunity to take time to consider their preferences, as treatment is heavily guideline-driven and preferably begun soon after diagnosis. We present lung cancer specialists' perspectives on how SDM can be incorporated in this complex decision and what role AI-based CDSSs may play in the treatment trajectory.

Chapter 6 continues the exploration of SDM in lung cancer, focusing on extensive stage small-cell lung cancer (ES-SCLC). Current guidelines recommend prophylactic cranial irradiation (PCI) to prevent brain metastasis, a treatment associated with heavy side-effects. We implement a simple SDM model to help ES-SCLC patients decide whether they wish to undergo PCI and measure their (and their clinicians') evaluation of the SDM process and treatment outcomes.

In Chapter 7, we study an example of successful SDM implementation using paper PDAs at Vejle Hospital, a cancer hospital in the region of Southern Denmark.

Chapter 8 concludes with a general review and discussion of our results in light of the current literature. Practical implications of our findings and avenues for future research are presented. 


\section{References}

1. Porter ME, Lee TH. The Strategy That Will Fix Health Care. :26.

2. Oshima Lee E, Emanuel EJ. Shared decision making to improve care and reduce costs. N. Engl. J. Med. 2013;368:6-8.

3. Institute of Medicine (US) Committee on Quality of Health Care in America. Crossing the Quality Chasm: A New Health System for the 21st Century [Internet]. Washington (DC): National Academies Press (US); 2001 [cited 2021 Jan 11]. Available from: http:/ / www.ncbi.nlm.nih.gov/books/NBK222274/

4. Mukherjee S. The emperor of all maladies: a biography of cancer. 1st Scribner trade paperback ed. New York: Scribner; 2011.

5. Pedersen JK, Engholm G, Skytthe A, Christensen K, Academy of Geriatric Cancer Research (AgeCare). Cancer and aging: epidemiology and methodological challenges. Acta Oncol. 2016;55:7-12.

6. Arnold M, Leitzmann M, Freisling H, Bray F, Romieu I, Renehan A, et al. Obesity and cancer: An update of the global impact. Cancer Epidemiol. 2016;41:8-15.

7. Arruebo M, Vilaboa N, Sáez-Gutierrez B, Lambea J, Tres A, Valladares M, et al. Assessment of the evolution of cancer treatment therapies. Cancers 2011;3:3279-330.

8. Morrison WB. Cancer Chemotherapy: An Annotated History: A History of Cancer Chemotherapy. J. Vet. Intern. Med. 2010;24:1249-62.

9. McCune JS. Rapid Advances in Immunotherapy to Treat Cancer. Clin. Pharmacol. Ther. 2018;103:540-4.

10. Crosby D. A roadmap for the early detection and diagnosis of cancer. 2020;21:3.

11. The Independent UK Panel on Breast Cancer Screening, Marmot MG, Altman DG, Cameron DA, Dewar JA, Thompson SG, et al. The benefits and harms of breast cancer screening: an independent review: A report jointly commissioned by Cancer Research UK and the Department of Health (England) October 2012. Br. J. Cancer 2013;108:2205-40. 
12. Weaver SJ, Jacobsen PB. Cancer care coordination: opportunities for healthcare delivery research. Transl. Behav. Med. 2018;8:503-8.

13. Shilo S, Rossman H, Segal E. Axes of a revolution: challenges and promises of big data in healthcare. Nat. Med. 2020;26:29-38.

14. Klerings I, Weinhandl AS, Thaler KJ. Information overload in healthcare: too much of a good thing? Z. Für Evidenz Fortbild. Qual. Im Gesundheitswesen 2015;109:285-90.

15. Will JF. A Brief Historical and Theoretical Perspective on Patient Autonomy and Medical Decision Making. Chest 2011;139:669-73.

16. Tauber AI. Patient autonomy and the ethics of responsibility. 2005;

17. Rothman DJ. The origins and consequences of patient autonomy: A 25year retrospective. Health Care Anal. 2001;9:255-64.

18. Beauchamp TL. Informed consent: its history, meaning, and present challenges. Camb. Q Healthc. Ethics 2011;20:515.

19. Elwyn G, Tilburt J, Montori V. The ethical imperative for shared decisionmaking. Eur. J. Pers. Centered Healthc. 2013;1:129.

20. Friedman AA, Letai A, Fisher DE, Flaherty KT. Precision medicine for cancer with next-generation functional diagnostics. Nat. Rev. Cancer 2015;15:747-56.

21. Ostermann J, Brown DS, van Til JA, Bansback N, Légaré F, Marshall DA, et al. Support Tools for Preference-Sensitive Decisions in Healthcare: Where Are We? Where Do We Go? How Do We Get There? Patient - Patient-Centered Outcomes Res. 2019;12:439-43.

22. Pomey M-P, Ghadiri DP, Karazivan P, Fernandez N, Clavel N. Patients as Partners: A Qualitative Study of Patients' Engagement in Their Health Care. PLOS ONE 2015;10:e0122499.

23. Kilbride MK, Joffe S. The New Age of Patient Autonomy: Implications for the Patient-Physician Relationship. JAMA 2018;320:1973-4.

24. Charles C, Gafni A, Whelan T. Decision-making in the physician-patient encounter: revisiting the shared treatment decision-making model. Soc. Sci. Med. 1982 1999;49:651-61. 
25. Beauchamp TL. Informed Consent: Its History, Meaning, and Present Challenges. Camb. Q. Healthc. Ethics 2011;20:515-23.

26. Childress JF, Childress MD. What does the evolution from informed consent to shared decision making teach us about authority in health care? AMA J. Ethics 2020;22:423-9.

27. Elwyn G, Edwards A, Thompson R, editors. Shared decision-making in health care: achieving evidence-based patient choice. Third edition. Oxford, United Kingdom; New York, NY, United States of America: Oxford University Press; 2016.

28. Singh JA, Sloan JA, Atherton PJ, Smith T, Hack TF, Huschka MM, et al. Preferred roles in treatment decision making among patients with cancer: a pooled analysis of studies using the Control Preferences Scale. Am. J. Manag. Care 2010;16:688-96.

29. Keij SM, van Duijn-Bakker N, Stiggelbout AM, Pieterse AH. What makes a patient ready for Shared Decision Making? A qualitative study. Patient Educ. Couns. 2021;104:571-7.

30. Elwyn G, Durand MA, Song J, Aarts J, Barr PJ, Berger Z, et al. A threetalk model for shared decision making: multistage consultation process. BMJ 2017;j4891.

31. Agency for Healthcare Research and Quality. The SHARE Approach-Using the Teach-Back Technique: A Reference Guide for Health Care Providers. 2014;

32. Rennke S, Yuan P, Monash B, Blankenburg R, Chua I, Harman S, et al. The SDM 3 Circle Model: A Literature Synthesis and Adaptation for Shared Decision Making in the Hospital. J. Hosp. Med. 2017;12:1001-8.

33. McGrail K, Karmally R, Kiwalker S. AHRQ SHARE approach training and implementation success story: Rochester regional health system uses shared decision making to improve patient care. 2016;

34. O'Connor M. Promoting Shared Decisionmaking: Focus on Students First. [Internet]. 2016;Available from: https://www.ahrq.gov/healthliteracy/professional-training/shared-decision/success/story-4.html

35. Shepherd HL, Barratt A, Trevena LJ, McGeechan K, Carey K, Epstein RM, et al. Three questions that patients can ask to improve the quality of infor- 
mation physicians give about treatment options: A cross-over trial. Patient Educ. Couns. 2011;84:379-85.

36. Rexwinkel R, Rippen H, Blokzijl-Boezeman IJM, de Klein Z, Walhof CM, van der Kraan J, et al. Evaluation of the "3 Good Questions" program for shared decision-making in pediatric medicine: a feasibility study. Eur. J. Pediatr. 2021;180:1235-42.

37. Bomhof-Roordink H, Gärtner FR, Stiggelbout AM, Pieterse AH. Key components of shared decision making models: a systematic review. BMJ Open 2019;9:e031763.

38. Diouf NT, Menear M, Robitaille H, Painchaud Guérard G, Légaré F. Training health professionals in shared decision making: Update of an international environmental scan. Patient Educ. Couns. 2016;99:1753-8.

39. Scholl I, LaRussa A, Hahlweg P, Kobrin S, Elwyn G. Organizational- and system-level characteristics that influence implementation of shared decisionmaking and strategies to address them - a scoping review. Implement. Sci. IS 2018;13:40.

40. Légaré F, Ratté S, Gravel K, Graham ID. Barriers and facilitators to implementing shared decision-making in clinical practice: update of a systematic review of health professionals' perceptions. Patient Educ. Couns. 2008;73:526-35.

41. Stacey D, Légaré F, Col NF, Bennett CL, Barry MJ, Eden KB, et al. Decision aids for people facing health treatment or screening decisions. Cochrane Database Syst. Rev. 2014;CD001431.

42. Pieterse AH, Stiggelbout AM, Montori VM. Shared Decision Making and the Importance of Time. JAMA 2019;322:25.

43. Couët N, Desroches S, Robitaille H, Vaillancourt H, Leblanc A, Turcotte $\mathrm{S}$, et al. Assessments of the extent to which health-care providers involve patients in decision making: a systematic review of studies using the OPTION instrument. Health Expect. 2015;18:542-61.

44. Joseph-Williams N, Elwyn G, Edwards A. Knowledge is not power for patients: A systematic review and thematic synthesis of patient-reported barriers and facilitators to shared decision making. Patient Educ. Couns. 2014;94:291-309. 
45. Fraenkel L, McGraw S. What are the Essential Elements to Enable Patient Participation in Medical Decision Making? J. Gen. Intern. Med. 2007;22:614-9.

46. Beaver K, Jones D, Susnerwala S, Craven O, Tomlinson M, Witham G, et al. Exploring the decision-making preferences of people with colorectal cancer. Health Expect. Int. J. Public Particip. Health Care Health Policy 2005;8:103-13.

47. Entwistle V, Prior M, Skea ZC, Francis JJ. Involvement in treatment decision-making: its meaning to people with diabetes and implications for conceptualisation. Soc. Sci. Med. 1982 2008;66:362-75.

48. Bastiaens H, Van Royen P, Pavlic DR, Raposo V, Baker R. Older people's preferences for involvement in their own care: a qualitative study in primary health care in 11 European countries. Patient Educ. Couns. 2007;68:33-42.

49. Ekdahl AW, Andersson L, Friedrichsen M. "They do what they think is the best for me." Frail elderly patients' preferences for participation in their care during hospitalization. Patient Educ. Couns. 2010;80:233-40.

50. Sainio C, Lauri S, Eriksson E. Cancer patients' views and experiences of participation in care and decision making. Nurs. Ethics 2001;8:97-113.

51. Bynum JPW, Barre L, Reed C, Passow H. Participation of very old adults in health care decisions. Med. Decis. Mak. Int. J. Soc. Med. Decis. Mak. 2014;34:216-30.

52. Montori VM, Breslin M, Maleska M, Weymiller AJ. Creating a Conversation: Insights from the Development of a Decision Aid. PLoS Med. 2007;4:e233.

53. Alam S, Elwyn G, Percac-Lima S, Grande S, Durand M-A. Assessing the acceptability and feasibility of encounter decision aids for early stage breast cancer targeted at underserved patients. BMC Med. Inform. Decis. Mak. 2016;16:147.

54. de Mik SML, Stubenrouch FE, Balm R, Ubbink DT. Development of three different decision support tools to support shared decision-making in vascular surgery. Patient Educ. Couns. 2021;104:282-9.

55. Stacey D, Légaré F, Lewis K, Barry MJ, Bennett CL, Eden KB, et al. Decision aids for people facing health treatment or screening decisions. 
Cochrane Database Syst. Rev. [Internet] 2017 [cited 2020 Dec 15];Available from: http:/ / doi.wiley.com/10.1002/14651858.CD001431.pub5

56. Guadagnoli E, Ward P. Patient participation in decision-making. Soc. Sci. Med. 1982 1998;47:329-39.

57. Tomko C, Davis KM, Luta G, Krist AH, Woolf SH, Taylor KL. A Comparison of Web-Based Versus Print-Based Decision Aids for Prostate Cancer Screening: Participants' Evaluation and Utilization. J. Gen. Intern. Med. 2015;30:33-42.

58. Estacio EV, Whittle R, Protheroe J. The digital divide: Examining sociodemographic factors associated with health literacy, access and use of internet to seek health information. J. Health Psychol. 2019;24:1668-75.

59. O'Donnell S, Cranney A, Jacobsen MJ, Graham ID, O'Connor AM, Tugwell $\mathrm{P}$. Understanding and overcoming the barriers of implementing patient decision aids in clinical practice. J. Eval. Clin. Pract. 2006;12:174-81.

60. Agoritsas T, Heen AF, Brandt L, Alonso-Coello P, Kristiansen A, Akl EA, et al. Decision aids that really promote shared decision making: the pace quickens. BMJ 2015;g7624.

61. Kawamoto K, Houlihan CA, Balas EA, Lobach DF. Improving clinical practice using clinical decision support systems: a systematic review of trials to identify features critical to success. Bmj 2005;330:765.

62. Bright TJ, Wong A, Dhurjati R, Bristow E, Bastian L, Coeytaux RR, et al. Effect of Clinical Decision-Support Systems: A Systematic Review. Ann. Intern. Med. 2012;157:29.

63. Beauchemin M, Murray MT, Sung L, Hershman DL, Weng C, Schnall R. Clinical decision support for therapeutic decision-making in cancer: A systematic review. Int. J. Med. Inf. 2019;130:103940.

64. Yang Q, Steinfeld A, Zimmerman J. Unremarkable AI: Fitting Intelligent Decision Support into Critical, Clinical Decision-Making Processes. Proc. 2019 CHI Conf. Hum. Factors Comput. Syst. 2019;1-11.

65. Kortteisto T, Komulainen J, Mäkelä M, Kunnamo I, Kaila M. Clinical decision support must be useful, functional is not enough: a qualitative study of computer-based clinical decision support in primary care. BMC Health Serv. Res. 2012;12:349. 
66. Liberati EG, Ruggiero F, Galuppo L, Gorli M, González-Lorenzo M, Maraldi $\mathrm{M}$, et al. What hinders the uptake of computerized decision support systems in hospitals? A qualitative study and framework for implementation. Implement. Sci. IS 2017;12:113.

67. Hallen SAM, Hootsmans NAM, Blaisdell L, Gutheil CM, Han PKJ. Physicians' perceptions of the value of prognostic models: the benefits and risks of prognostic confidence. Health Expect. Int. J. Public Particip. Health Care Health Policy 2015;18:2266-77.

68. Lambin P, Zindler J, Vanneste BGL, De Voorde LV, Eekers D, Compter I, et al. Decision support systems for personalized and participative radiation oncology. Adv. Drug Deliv. Rev. 2017;109:131-53.

69. Katz SJ, Belkora J, Elwyn G. Shared decision making for treatment of cancer: challenges and opportunities. J. Oncol. Pract. 2014;10:206-8.

70. Irizarry T, DeVito Dabbs A, Curran CR. Patient Portals and Patient Engagement: A State of the Science Review. J. Med. Internet Res. 2015;17:e148.

71. Jayaratne M, Nallaperuma D, De Silva D, Alahakoon D, Devitt B, Webster $\mathrm{KE}$, et al. A data integration platform for patient-centered e-healthcare and clinical decision support. Future Gener. Comput. Syst. 2019;92:996-1008.

72. Galesic M, Garcia-Retamero R. Using Analogies to Communicate Information about Health Risks: Analogies for health communication. Appl. Cogn. Psychol. 2013;27:33-42.

73. Operskalski JT, Barbey AK. Risk literacy in medical decision-making. Science 2016;352:413-4.

74. Moxey A, O'Connell D, McGettigan P, Henry D. Describing treatment effects to patients. J. Gen. Intern. Med. 2003;18:948-59.

75. Almashat S, Ayotte B, Edelstein B, Margrett J. Framing effect debiasing in medical decision making. Patient Educ. Couns. 2008;71:102-7.

76. Burke RE, Leonard C, Lee M, Ayele R, Cumbler E, Allyn R, et al. Cognitive Biases Influence Decision-Making Regarding Postacute Care in a Skilled Nursing Facility. J. Hosp. Med. 2020;15:22-7. 



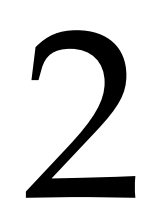

\section{The benefits and challenges of using patient decision aids to support shared decision-making in health-care}

Ankolekar, A., Dekker, A., Fijten, R., \& Berlanga, A.

JCO Clinical Cancer Informatics (2018) 2, 1-10. 


\section{Abstract}

Shared decision making (SDM) and patient-centered care require patients to actively participate in the decision-making process. Yet with the increasing number and complexity of cancer treatment options, it can be a challenge for patients to evaluate clinical information and make risk- benefit trade-offs to choose the most appropriate treatment. Clinicians face time constraints and communication challenges, which can further hamper the SDM process. In this article, we review patient decision aids (PDAs) as a means of supporting SDM by presenting clinical information and risk data to patients in a format that is accessible and easy to understand. We outline the benefits and limitations of PDAs as well as the challenges in their development, such as a lengthy and complex development process and implementation obstacles. Lastly, we discuss future trends and how change on multiple levels - PDA developers, clinicians, hospital administrators, and health care insurers - can support the use of PDAs and consequently SDM. Through this multipronged approach, patients can be empowered to take an active role in their health and choose treatments that are in line with their values. 


\subsection{Introduction}

The health care landscape is undergoing rapid advances in the amount and type of clinical data collected, analyzed, and shared. In addition, patients and consumers now have access to a greater range of information through the Internet and are willing and able to take on a more active role in their health. These trends are leading to the evolution of $\mathrm{P} 4$ medicine-health care that is personalized, preventive, predictive, and participatory [1]. P4 medicine has the potential to improve patient outcomes and clinical interactions in which both the patient and clinician have access to data that are accurate, relevant, and evidence based. In addition, $\mathrm{P} 4$ medicine may reduce the increasing costs of health care, for instance, by stratifying patients according to their personal characteristics, which allows for more targeted therapies and potentially less invasive and expensive treatment decisions [2].

Operationalizing P4 medicine will involve confronting both technological and societal challenges. The complexity of decision making in oncology forces patients and clinicians to consider the pros and cons of an increasing number of treatment options [3]. In addition, patients evaluate their treatment options differently from their clinicians. For instance, patients with breast cancer were found to consider multiple factors in their decision making, such as the impact on daily life, maintaining fertility, and adverse effects, whereas clinicians tended to focus more on survival [4]. Furthermore, when faced with making choices under conditions of uncertainty, both patients and clinicians are susceptible to bias and reliance on heuristics [5]. Many patients face difficulty in forecasting their future [6] and often lack the skills to interpret risk figures and probabilities $[7,8]$. Patients and clinicians will need new ways of collaborating to evaluate all relevant pieces of evidence and personal preferences to make optimal choices.

The shared decision-making (SDM) paradigm emerged as a way to consolidate the wishes of both parties-the patient and the clinician [9]. Under the traditional paternalistic model, information flows one way from the clinician to the patient, deliberation on treatment options 
largely involves the main clinician and other medical staff, and the final decision is made by the clinician with little or no input from the patient. High-quality SDM is based on three pillars [10]: two-way information sharing between the patient and clinician, deliberation on the basis of the patient's preferences, and the involvement of both the patient and clinician in the final decision-making process. SDM has been associated with less decisional regret and lower anxiety about treatment choices, better health outcomes, and greater patient satisfaction [11]; however, as with any new innovation, implementing SDM requires adjustments by health care professionals to overcome barriers, such as time constraints, a perceived lack of applicability in certain cases, and cultural changes [12].

Patient decision aids (PDAs) have been developed as a way to support patient participation in the decision-making process and to increase the likelihood of reaching a treatment decision that is both clinically sound and in line with patients' values. A PDA is any tool-booklet, Web site, or application-that helps the patient understand his or her treatment options to make an informed decision [13]. A well-designed PDA provides balanced information on treatment benefits and risks and can help patients identify and reflect on their personal preferences. PDAs are particularly useful in disease areas that have multiple treatment options with different outcomes and adverse effects [14].

The objective of this review is to gather and assess evidence of the role of PDAs in supporting the SDM process. We begin by reviewing the types of PDAs and their benefits and limitations. We then describe the development process and its associated challenges. We end with a brief exploration of future trends in light of the changing technological and social landscape and what is needed to build a stronger evidence base to aid in the development and implementation of PDAs. 


\subsection{Benefits and limitations of PDAs}

PDAs can guide a variety of health care decisions that range from screening decisions and treatment decisions to post-treatment followup (an alphabetical list of PDAs according to disease type has been created by the Ottawa Hospital Research Institute [15]). A typical PDA contains the following: information about the disease type; treatment options; risks, benefits, and uncertainties associated with each option; and a form of value clarification exercise in which patients are asked to make choices the basis of the trade-offs between the treatment options and risks. The purpose of value clarification is to allow patients to reflect on what aspects of the treatment options matter most to them so that it is easier for clinicians to engage with patients and guide the decision-making process toward the most ideal outcome.

Use of PDAs has been shown to improve care quality on several dimensions, per evidence from a Cochrane review of 105 randomized controlled trials (RCTs) across 10 countries [16]. In 52 RCTs, patients who used a PDA scored higher on knowledge tests than those who received standard care, and in 17 RCTs were able to interpret risks more accurately. By helping patients become more informed, PDAs can also improve the quality of the clinical interaction and communication between patients and clinicians; five RCTs report that PDA users demonstrated statistically significant increases in OPTION (observing patient involvement in decision making) scores, a method used to evaluate clinical interactions on the basis of SDM principles. Furthermore, patients took on a more active role in decision making when they used a PDA compared with standard care, as demonstrated in 15 RCTs.

Whereas there is abundant evidence of the clinical benefits of PDAs, a considerable gap exists in the literature with regard to their economic viability. Development and implementation involves upfront costs - time, labor, and resources. One feasibility study summed up the time and costs associated with one staff member delivering a PDA to 268 patients over a 5-month period and found that the total time spent in the clinical encounter, including time spent reviewing pa- 
tients' medical records, amounted to an additional 292 hours-65 minutes per patient on average [17]. There is a lack of similar studies to corroborate this finding, although evidence from 10 RCTs suggests that PDAs increase consultation times slightly [18].

In contrast, other evidence asserts that PDAs have the potential to reduce costs in the long term as patients may choose less aggressive treatments when provided with more information about each treatment [11,19]. Indeed, up to $20 \%$ of patients who use PDAs choose less-invasive procedures and more conservative therapies [20]. For instance, use of a PDA for hip and knee replacement decisions resulted in $26 \%$ fewer hip replacements, $38 \%$ fewer knee replacements, and an overall cost reduction of up to $21 \%$ over a 6 -month period [21]. Another study that was prepared for the Commonwealth Fund predicted that the implementation of PDAs in 11 treatment areas could result in net savings of more than $\$ 7$ billion over a 10-year period [22]. Yet a systematic review of the economic evaluation of PDAs demonstrates limited evidence of cost effectiveness over the long term [23-25]. More evidence is needed to demonstrate potential costs savings.

Lastly, although most PDAs follow a similar format, they can vary widely in terms of the way the content is presented [26-28], and there is little consensus on which representation is most effective. For instance, developers might assume that it is better to include as much information as possible; however, too much information can lead to cognitive overload and contribute to sub-optimal decision making [29]. In addition, most PDAs are designed for the patient to use at home and it is not always possible to monitor the uptake of information and determine the effect in consultation $[30,31]$. Together with the fact that PDAs are frequently outdated [32], it is a challenge to develop and implement high-quality PDAs that can be routinely used in practice [33]. 


\subsection{The development of PDAs}

The International Patient Decision Aid Standards (IPDAS) were established in 2003 by an international team of researchers and practitioners to create consensus on quality standards between PDA developers around the world. The proposed guidelines for developing PDAs are summarized in Figure 2.1. The first step of this process is to determine the scope of the decision aid, such as disease area, decision to be made, and target population. Next, a steering group is formed that consists of stakeholders who have expertise in the decision area, such as clinicians, patients, patient advocacy groups, researchers, or policy makers. The steering group oversees the development process, which consists of the following elements: assessing patients' decisional needs, systematically reviewing and synthesizing the literature, developing a distribution plan for the PDA, developing prototypes, and conducting alpha and beta testing of prototypes.

Determining patients' decisional needs is a foundational step in developing an effective PDA for several reasons. First, patients evaluate their treatment options differently than clinicians [35]. In addition, patient populations are heterogeneous and one patient's informational needs may differ significantly from those of another [36]. As a result, it is important to gain a range of perspectives on the type and quantity of information that patients need to evaluate their options. Patients, clinicians, and advocacy groups can be valuable sources of data and are typically engaged through focus groups, surveys, and interviews.

Once the main decisional needs have been established and incorporated into the initial prototypes, these prototypes must be tested. IPDAS guidelines recommend initial alpha testing among patients and clinicians in a laboratory setting, followed by broader beta testing in a real clinical practice setting. Finally, implementation must be evaluated using appropriate performance metrics, for example, the extent of use of the decision tool (i.e., the number of patients who used the tool as a proportion of those who were eligible to use it) or improvements in the quality of decision support provided by the clinician as 
measured by the Decision Support Analysis Tool (DSAT-10) [37].

IPDAS guidelines represent a standardized development process that can reduce variability in the quality of PDAs; however, questions have been raised about its validity in practice. For instance, as a result of a lack of input from the field of decision science, little is known about which specific components of the IPDAS guidelines best facilitate the decision-making process [38]. Gaps have also been identified in the evidence base on presenting probabilistic information [39]. Furthermore, a well-designed PDA may not improve clinical outcomes unless it is properly implemented in clinical practice. Consequently, there are challenges for PDA developers, clinicians, hospital administrators, and the wider health care system. 


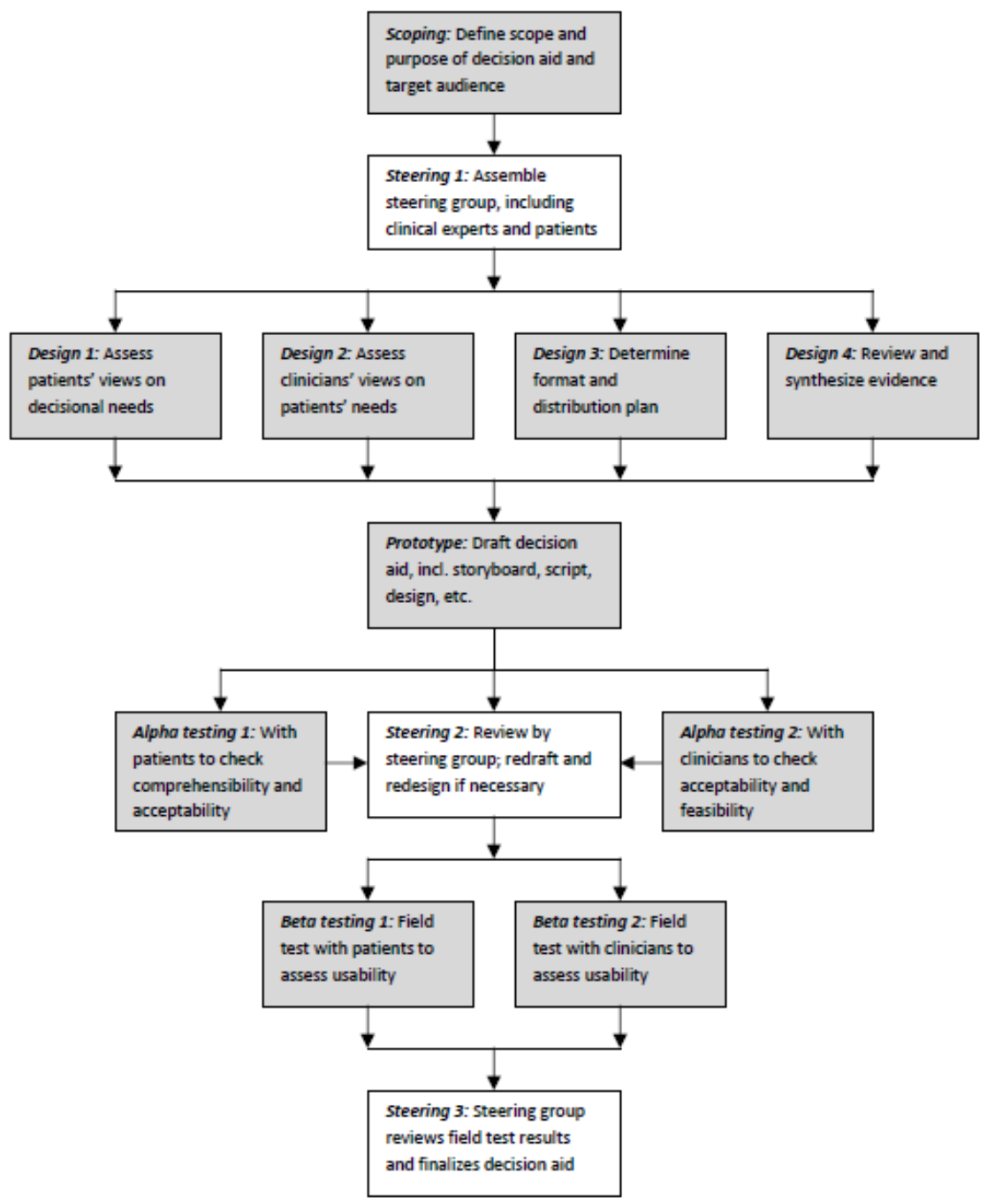

Figure 2.1: An International Patient Decision Aid Standards model for the development process for a patient decision aid. Reprinted with permission from Coulter et al [34] 


\subsection{Challenges in the development and implementation of PDAs}

The main challenges for PDA developers arise in the improvement of PDA usability, accuracy, and acceptability. Usability challenges include presenting information, particularly on risks and probabilities, in a way that is easy to understand by the general population. Low numeracy levels affect the ability of patients to interpret risk information correctly [40], and numeracy tends to decline with age [41,42]. PDA developers need to consider evidence-based methods of different ways to communicate risks other than numerically-for instance, through visual aids, graphs, and patient narratives [43]. An additional challenge for developers is designing PDAs that are accepted by clinicians and do not increase their workloads, as time constraints are the most oftencited barrier to SDM and PDA use [44]. Clinicians are also less likely to use or recommend a PDA if they feel it does not contain accurate data. User-centered design can help mitigate these factors [45] and is based on the principle that an innovation is more likely to be successful when its users are consulted in an iterative process [46-48], in contrast to the relatively linear design approach in current IPDAS guidelines. There is little guidance in the current literature on user-centered PDA design, although developers can seek insights from its application in other fields that involve human-computer interaction [49-51].

Clinicians may need to develop new skills in guiding decisional talk that acknowledge the expanded role of the patient in the SDM process. Prior findings suggest two main skill categories: relational competencies (creating quality interactions between clinicians and patients) and risk communication competencies (helping patients understand treatment options and risks) [52]. Relational competencies, such as effective communication, can be developed through training courses, elearning, or virtual patients to familiarize doctors with the core components of SDM. Risk communication competencies involve developing suitable ways to conceptualize the treatment-associated probabilities within decisional talk beyond the contents of PDAs. 
Hospital administrators may also play a key role in integrating PDAs and SDM into the clinical workflow to achieve the highest degree of effectiveness [53]. As the use of electronic health records (EHRs) becomes commonplace [54], integrating PDAs into the hospital's EHR system to varying degrees could be a key step [55]. For instance, the EHR could simply remind clinicians to give patients access to the PDA. One step further, the output of the PDA could be sent automatically to the EHR, which would allow the clinician to see the patient's results, preferences, and any questions to be addressed in advance of the next consultation. The EHR system could also keep track of which data have been filled in and what is missing, providing a more complete overview of the patient's clinical and personal information. Implementing these changes requires developers to collaborate with the hospital's information technology department to integrate the PDA into the existing EHR system. Roles and responsibilities must also be clearly defined so that the PDA can be routinely maintained and updated to reflect the latest evidence.

Finally, although SDM is largely centered on the interaction between health care professionals and patients, other parties outside the clinical setting can also support its implementation. Health care insurers can create incentives for the use of decision support systems by providing reimbursement for the use of decision aids or by making SDM a prerequisite for reimbursing certain procedures. Organizations for patients with cancer can be instrumental in raising awareness among patients and the public about their potential role in the clinical decisionmaking process as well as in the promotion of PDAs. Finally, the evaluation and dissemination of SDM outcomes can be seen as a quality indicator for care providers and could increase the acceptance of PDAs by health care professionals.

\subsection{Future trends}

The issues outlined so far reveal the need for a stronger evidence base regarding the development, implementation, and cost effectiveness of 
PDAs. Measurement remains a methodological challenge [56], and the validity of current evaluation measures, such as patient satisfaction and decisional conflict, has not yet been definitively established [57], possibly because of a lack of patient involvement in the development of these instruments. Such methods as cognitive interviewing may be valuable for the development of more patient-centered outcome measurements [58]. Furthermore, existing models for economic evaluation of PDAs may need to be extended to account for long-term effects and quality-adjusted life-years [59].

Developers, patients, and health care professionals will also need to adapt to the rapidly changing technological landscape. As computing power and the amount of data generated continue to increase, data analytics and machine learning techniques will allow researchers to identify patterns in medical data that can help predict treatment outcomes [60]. These prediction models can be used to quantify the likelihood of a specific outcome for a specific patient on the basis of individual risk factors, and can ultimately support personalized decision making [61]. When aggregated and analyzed over large populations, these data could allow individual patients to compare the choices of patients who are similar to them. Key challenges for developers, clinicians, and hospital administrators are to develop a standardized method of aggregating data across different hospital systems, regions, and countries while protecting patient privacy [62] and embedding the resulting prediction models into PDAs. In addition, mobile technology has become more accessible than Web-based applications [63], and this trend will require developers to adapt PDAs to multiple platforms-smartphones and tablets-and across different patient demographics, particularly elderly patients [64-65] and those with lower health literacy [66].

\subsection{Conclusion}

PDAs have the potential to improve health outcomes by presenting complex medical information in a form that allows patients and clin- 
icians to collaborate more effectively. Through multimedia-images, videos, and animations, PDAs can help patients understand disease information and treatment procedures and their risks and benefits. Interactive PDAs also allow patients to weigh different treatment options to clarify their personal preferences. Results can be used to guide clinical interactions in a more balanced process in which patients are better informed and clinicians take into account patients' values. Good design is a crucial element for the realization of this potential.

User-centered design methods can enhance PDA quality and acceptability and improve the likelihood of successful adoption. Patients, clinicians, nurses, hospital administrators, and researchers must be engaged in a multidisciplinary process, as sound decision making extends beyond the patient-clinician interaction. In addition to developing high-quality PDAs, the environment needs to be conducive to their implementation. An understanding of the existing workflow within the hospital is needed so that the PDA can be integrated smoothly. In addition, decision makers must be incentivized to engage in SDM and use PDAs. This can be stimulated directly through reimbursement schemes and indirectly by systematic measurement and dissemination of PDA use outcomes to increase the evidence base. The increasing prominence of data analytics in medical decision making requires a paradigm shift in the health care industry on multiple levels-clinical, administrative, policy, and service mind oriented. 


\section{References}

1. Hood L, Friend SH: Predictive, personalized, preventive, participatory (P4) cancer medicine. Nat Rev Clin Oncol 8:184-187, 2011

2. Hood L, Galas D: P4 medicine: Personalized, predictive, preventive, participatory-A change of view that changes everything. https://cra.org/ccc/wp-content/uploads/sites/2/2015/05/P4_-

Medicine.pdf

3. Reyna VF, Nelson WL, Han PK, et al: Decision making and cancer. Am Psychol 70:105-118, 2015

4. Lee CN, Dominik R, Levin CA, et al: Development of instruments to measure the quality of breast cancer treatment decisions. Health Expect 13:258272,2010

5. Tversky A, Kahneman D: Advances in prospect theory: Cumulative representation of uncertainty. J Risk Uncertain 5:297-323, 1992

6. Redelmeier DA, Rozin P, Kahneman D: Understanding patients' decisions. Cognitive and emotional perspectives. JAMA 270:72-76, 1993

7. Mazur DJ, Hickam DH: Patient interpretations of terms connoting low probabilities when communicating about surgical risk. Theor Surg 8:143-145, 1993

8. Woloshin S, Schwartz LM, Moncur M, et al: Assessing values for health: Numeracy matters. Med Decis Making 21:382-390, 2001

9. Charles C, Gafni A, Whelan T: Decision-making in the physician-patient encounter: Revisiting the shared treatment decision-making model. Soc Sci Med 49:651-661, 1999

10. Charles C, Gafni A, Whelan T: Shared decision-making in the medical encounter: What does it mean? (or it takes at least two to tango). Soc Sci Med 44:681-692, 1997

11. Oshima Lee E, Emanuel EJ: Shared decision making to improve care and reduce costs. N Engl J Med 368:6-8, 2013

12. Gravel K, Légaré F, Graham ID: Barriers and facilitators to implementing shared decision-making in clinical practice: A systematic review of health professionals' perceptions. Implement Sci 1:16, 2006 
13. Ruland CM: Improving patient safety through informatics tools for shared decision making and risk communication. Int J Med Inform 73:551-557, 2004

14. O'Connor A: Using patient decision aids to promote evidence-based decision making. ACP J Club 135:A11-A12, 2001

15. The Ottawa Hospital Research Institute: Alphabetical list of decision aids by topic. https:/ / decisionaid.ohri.ca/AZlist.html

16. Stacey D, Légaré F, Lewis K, et al: Decision aids for people facing health treatment or screening decisions. Cochrane Database Syst Rev 4:CD001431, 2017

17. Miller KM, Brenner A, Griffith JM, et al: Promoting decision aid use in primary care using a staff member for delivery. Patient Educ Couns 86:189194,2012

18. Bekker HL, Hewison J, Thornton JG: Applying decision analysis to facilitate informed decision making about prenatal diagnosis for Down syndrome: A randomised controlled trial. Prenat Diagn 24:265-275, 2004

19. Knops AM, Legemate DA, Goossens A, et al: Decision aids for patients facing a surgical treatment decision: A systematic review and meta-analysis. Ann Surg 257:860-866, 2013

20. Stacey D, Bennett CL, Barry MJ, et al: Decision aids for people facing health treatment or screening decisions. Cochrane Database Syst Rev 2011:CD001431, 2011

21. Arterburn D, Wellman R, Westbrook E, et al: Introducing decision aids at Group Health was linked to sharply lower hip and knee surgery rates and costs. Health Aff (Millwood) 31:2094- 2104, 2012

22. Schoen C, Guterman S, Shih A, et al: Bending the Curve: Options for Achieving Savings and Improving Value in US Health Spending. New York, NY, The Commonwealth Fund, 2007

23. Trenaman L, Bryan S, Bansback N: The cost-effectiveness of patient decision aids: A systematic review. Healthc (Amst) 2:251-257, 2014

24. Kennedy AD, Sculpher MJ, Coulter A, et al: Effects of decision aids for menorrhagia on treatment choices, health outcomes, and costs: A randomized controlled trial. JAMA 288:2701-2708, 2002 
25. Walsh T, Barr PJ, Thompson R, et al: Undetermined impact of patient decision support interventions on healthcare costs and savings: Systematic review. BMJ 348:g188, 2014

26. Raats CJ, van Veenendaal H, Versluijs MM, et al: A generic tool for development of decision aids based on clinical practice guidelines. Patient Educ Couns 73:413-417, 2008

27. O'Connor AM, Drake ER, Fiset V, et al: The Ottawa patient decision aids. Eff Clin Pract 2:163-170, 1999

28. Elwyn G, Kreuwel I, Durand MA, et al: How to develop web-based decision support interventions for patients: A process map. Patient Educ Couns 82:260-265, 2011

29. Peters E, Klein W, Kaufman A, et al: More is not always better: Intuitions about effective public policy can lead to unintended consequences. Soc Issues Policy Rev 7:114-148, 2013

30. Hargraves I, Montori VM: Decision aids, empowerment, and shared decision making. BMJ 349:g5811, 2014

31. Agoritsas T, Heen AF, Brandt L, et al: Decision aids that really promote shared decision making: The pace quickens. BMJ 350:g7624, 2015

32. Montori VM, LeBlanc A, Buchholz A, et al: Basing information on comprehensive, critically appraised, and up-to-date syntheses of the scientific evidence: A quality dimension of the International Patient Decision Aid Standards. BMC Med Inform Decis Mak 13:S5, 2013

33. Elwyn G, Légaré F, van der Weijden T, et al: Arduous implementation: Does the Normalisation Process Model explain why it's so difficult to embed decision support technologies for patients in routine clinical practice. Implement Sci 3:57, 2008

34. Coulter A, Stilwell D, Kryworuchko J, et al: A systematic development process for patient decision aids. BMC Med Inform Decis Mak 13:S2, 2013 (suppl 2)

35. Turner S, Maher EJ, Young T, et al: What are the information priorities for cancer patients involved in treatment decisions? An experienced surrogate study in Hodgkin's disease. Br J Cancer 73:222-227, 1996 
36. Feldman-Stewart D, Brundage MD, Nickel JC, et al: The information required by patients with early-stage prostate cancer in choosing their treatment. BJU Int 87:218-223, 2001

37. Butow P, Juraskova I, Chang S, et al: Shared decision making coding systems: How do they compare in the oncology context? Patient Educ Couns 78:261-268, 2010

38. Bekker HL: The loss of reason in patient decision aid research: Do checklists damage the quality of informed choice interventions? Patient Educ Couns 78:357-364, 2010

39. McDonald H, Charles C, Gafni A: Assessing the conceptual clarity and evidence base of quality criteria/standards developed for evaluating decision aids. Health Expect 17:232-243, 2014

40. Woloshin S, Schwartz LM, Black WC, et al: Women's perceptions of breast cancer risk: How you ask matters. Med Decis Making 19:221-229, 1999

41. Peters E, Diefenbach MA, Hess TM, et al: Age differences in dual information-processing modes: Implications for cancer decision making. Cancer 113:3556-3567, 2008

42. Reyna VF, Nelson WL, Han PK, et al: How numeracy influences risk comprehension and medical decision making. Psychol Bull 135:943-973, 2009

43. Trevena LJ, Zikmund-Fisher BJ, Edwards A, et al: Presenting quantitative information about decision outcomes: A risk communication primer for patient decision aid developers. BMC Med Inform Decis Mak 13:S7, 2013 (suppl 2)

44. Légaré F, Ratté S, Gravel K, et al: Barriers and facilitators to implementing shared decision- making in clinical practice: Update of a systematic review of health professionals' perceptions. Patient Educ Couns 73:526-535, 2008

45. Witteman HO, Dansokho SC, Colquhoun H, et al: User-centered design and the development of patient decision aids: Protocol for a systematic review. Syst Rev 4:11, 2015

46. Abras C, Maloney-Krichmar D, Preece J: User-centered design, in Bainbridge W (ed): Encyclopedia of Human-Computer Interaction. Thousand Oaks, CA, Sage Publications, 2004, pp 445-456 
47. Kuniavsky M: Observing the User Experience: A Practitioner's Guide to User Research. New York, NY, Elsevier, 2003

48. Garrett JJ: The Elements of User Experience: User-Centered Design for the Web and Beyond. New York, NY, Pearson Education, 2010

49. Lindgaard G, Chattratichart J: Usability testing: What have we overlooked?Proc SIGCHI Conference on Human Factors in Computing Systems2007:1415-1424, 2007

50. Bastien JM: Usability testing: A review of some methodological and technical aspects of the method. Int J Med Inform 79:e18-e23, 2010

51. Vredenburg K, Mao J-Y, Smith PW, et al: A survey of user-centered design practice. Proc SIGCHI Conference on Human Factors in Computing Systems2007:471-478, 2002

52. Légaré F, Moumjid-Ferdjaoui N, Drolet R, et al: Core competencies for shared decision making training programs: Insights from an international, interdisciplinary working group. J Contin Educ Health Prof 33:267-273, 2013

53. Karsh B-T: Clinical practice improvement and redesign: How change in workflow can be supported by clinical decision support. Rockville, MD, Agency for Healthcare Research and QualityPublication 09-0054-EF, 2009. https://healthit.ahrq.gov/sites/default/files/docs/biblio/090054-EF-Updated_0.pdf

54. Hsiao C-J, Jha AK, King J, et al: Office-based physicians are responding to incentives and assistance by adopting and using electronic health records. Health Aff (Millwood) 32:1470-1477, 2013

55. Lenert L, Dunlea R, Del Fiol G, et al: A model to support shared decision making in electronic health records systems. Med Decis Making 34:987-995, 2014

56. Härter M, van der Weijden T, Elwyn G: Policy and practice developments in the implementation of shared decision making: An international perspective. Z Evid Fortbild Qual Gesundhwes 105:229-233, 2011

57. Scholl I, Koelewijn-van Loon M, Sepucha K, et al: Measurement of shared decision making: A review of instruments. Z Evid Fortbild Qual Gesundhwes 105:313-324, 2011 
58. Barr PJ, Elwyn G: Measurement challenges in shared decision making: Putting the 'patient' in patient-reported measures. Health Expect 19:993-1001, 2016

59. Ara R, Brazier J, Sculpher $M$, et al: A framework for conducting economic evaluations when using patient decision aids in health care decision making. Sheffield, United Kingdom, University of Sheffield EEPRU Research Report 049, 2015. http://www.eepru.org.uk/wp-content/ uploads/2017/11/eepru-report-framework-for-ecomonic-evalutions-repdas-2015-049.pdf

60. Kourou K, Exarchos TP, Exarchos KP, et al: Machine learning applications in cancer prognosis and prediction. Comput Struct Biotechnol J 13:8-17, 2014

61. Vickers AJ: Prediction models in cancer care. CA Cancer J Clin 61:315-326, 2011

62. Jensen PB, Jensen LJ, Brunak S: Mining electronic health records: Towards better research applications and clinical care. Nat Rev Genet 13:395405,2012

63. Abbasgholizadeh Rahimi S, Menear M, Robitaille H, et al: Are mobile health applications useful for supporting shared decision making in diagnostic and treatment decisions? Glob Health Action 10:1332259, 2017 (suppl 3)

64. van Weert JC, van Munster BC, Sanders R, et al: Decision aids to help older people make health decisions: A systematic review and meta-analysis. BMC Med Inform Decis Mak 16:45, 2016

65. Heart T, Kalderon E: Older adults: Are they ready to adopt health-related ICT? Int J Med Inform 82:e209-e231, 2013

66. Brabers AE, Rademakers JJ, Groenewegen PP, et al: What role does health literacy play in patients' involvement in medical decision-making? PLoS One 12:e0173316, 2017 



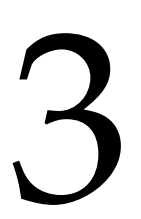

\section{Development and validation of a patient decision aid for prostate cancer therapy: from paternalistic towards participative shared decision-making}

Ankolekar, A., Vanneste, B. G., Bloemen-van Gurp, E., Van Roermund, J. G., van Limbergen, E. J., Van de Beek, K., Marcelissen, T., Zambon, V., Oelke, M., Dekker, A., Roumen, C., Lambin, P., Berlanga, A., \& Fijten, R.

BMC Medical Informatics and Decision Making (2019), 19(1), 1-11. 


\section{Abstract}

\section{Background}

Patient decision aids (PDAs) can support the treatment decision making process and empower patients to take a proactive role in their treatment pathway while using a shared decision-making (SDM) approach making participatory medicine possible. The aim of this study was to develop a PDA for prostate cancer that is accurate and user-friendly.

\section{Methods}

We followed a user-centered design process consisting of five rounds of semi-structured interviews and usability surveys with topics such as informational/decisional needs of users and requirements for PDAs. Our user-base consisted of 8 urologists, 4 radiation oncologists, 2 oncology nurses, 8 general practitioners, 19 former prostate cancer patients, 4 usability experts and 11 healthy volunteers.

\section{Results}

Informational needs for patients centered on three key factors: treatment experience, post-treatment quality of life, and the impact of side effects. Patients and clinicians valued a PDA that presents balanced information on these factors through simple understandable language and visual aids. Usability questionnaires revealed that patients were more satisfied overall with the PDA than clinicians; however, both groups had concerns that the PDA might lengthen consultation times ( 42 and $41 \%$, respectively). The PDA is accessible on http:/ / beslissamen.nl.

\section{Conclusions}

User-centered design provided valuable insights into PDA requirements but challenges in integrating diverse perspectives as clinicians focus on clinical outcomes while patients also consider quality of life. Nevertheless, it is crucial to involve a broad base of clinical users in order to better understand the decision-making process and to develop a PDA that is accurate, usable, and acceptable. 


\subsection{Background}

Prostate cancer (PCa) is the second most frequently diagnosed cancer type in males, with a rising global incidence rate and disease burden $[1,2]$. Making a treatment decision is often a challenging process for low-risk PCa patients as it involves trade-offs between the diverse side effects of four main treatment options with equal outcomes (radical prostatectomy, brachytherapy, external beam radiotherapy and active surveillance [3]). Since patients have differing lifestyles, values and educational backgrounds, a treatment acceptable to one patient may not be to another. In preference-sensitive conditions like $\mathrm{PCa}$, patients can benefit from shared decision-making (SDM) which involves reliable communication and collaboration between clinicians and patients to discuss and balance all available treatment options [4-6]. SDM is rarely used in the field of (radiation) oncology despite the evidence in favor of it $[5,6]$.

Participating in SDM requires that the patient is well informed about all available treatment options and is able to express his personal values and preferences in consultations with the clinician. However, studies have shown that most patients receive insufficient information during consultations [7], which can result in sub-optimal choices that negatively impact their post-treatment quality of life $[8,9]$. Furthermore, PCa patients benefit from a multidisciplinary approach of urologists and radiation oncologists [10]; specialists from these departments may have limited knowledge of treatments outside their area, leading them to provide unbalanced information. This is supported by evidence that a disproportionately large number of patients with low/intermediate risk PCa undergo surgery even though radiotherapy offers similar survival outcomes [11, 12].

A patient decision aid (PDA) can be a valuable tool in providing information on different treatment options and improving the quality of care as well as reducing unnecessary procedures, complications and costs [13]. Patients who use PDAs are better informed, experience less decisional conflict and report more realistic expectations of treatment 
outcomes [14]. Yet, their implementation in practice is hampered by several barriers perceived by clinicians, such as a lack of confidence in the PDA, lack of training in using it as an SDM tool, and concerns that it will take too much time to use [15].

User-centered design has been proposed as a way to make PDAs more suitable for clinical implementation [16]. In contrast to a standard development process in which informational needs assessment and prototype testing are carried out as separate processes, user-centered design is an iterative process; end-users' feedback shapes not only the content but also the design of the prototypes, resulting in innovations that are likely to be safer, more accurate and easier to use [17].

The goal of this study was to develop a web-based PDA for PCa patients in the Netherlands based on user-centered design principles. The PDA aims to provide accurate and balanced information about the main treatment options and a means for patients to discover and communicate their preferences with clinicians during the consultation.

\subsection{Methods}

\subsubsection{Participants}

In order to develop a fully validated, freely accessible, web-based PDA for PCa patients with information on four common and generally accepted PCa treatments (surgery, brachytherapy, external radiation, and active surveillance), we recruited four user groups:

1. Clinicians specializing in all four treatment areas: 4 radiation oncologists, 8 urologists, 2 oncology nurses and 8 general practitioners (GPs).

2. Nineteen former PCa patients who had fully undergone one of the four treatments.

3. Four usability evaluators with experience in web design, usability engineering and user experience, selected from the Informa- 
tion and Computer Science Department of Utrecht University. The evaluators had no clinical background.

4. Eleven volunteers with no history of PCa diagnosis or treatment. The volunteers had varying educational backgrounds and familiarity with technology, meant to represent the (typically elderly) PCa patient population [18].

The Internal Review Board (IRB) of Maastro Clinic reviewed and approved this study, and written informed consent was obtained from all respondents.

\subsubsection{Development process}

The development process was based on a combination of the International Patient Decision Aid Standards (IPDAS) [19] and principles of user-centered design [17]. As recommended in IPDAS guidelines, we conducted interviews with clinicians and patients to determine informational/decision needs. Based on the feedback from the user groups, we developed, tested and revised prototypes of the PDA over successive rounds. To this end, we used several interviewing and prototyping methods to test the validity and usability of the prototypes, as described below and in Figure 3.1.

\section{Rounds 1-4}

In the first four rounds, we performed semi-structured interviews and usability surveys in Dutch with patients and clinicians.

Semi-structured interviews

Semi-structured interviews were performed to assess the type and amount of information needed for decision-making. The interviews included the following themes: current information provision, the deliberation and decision-making process, patient preferences, and the potential for decision support. 


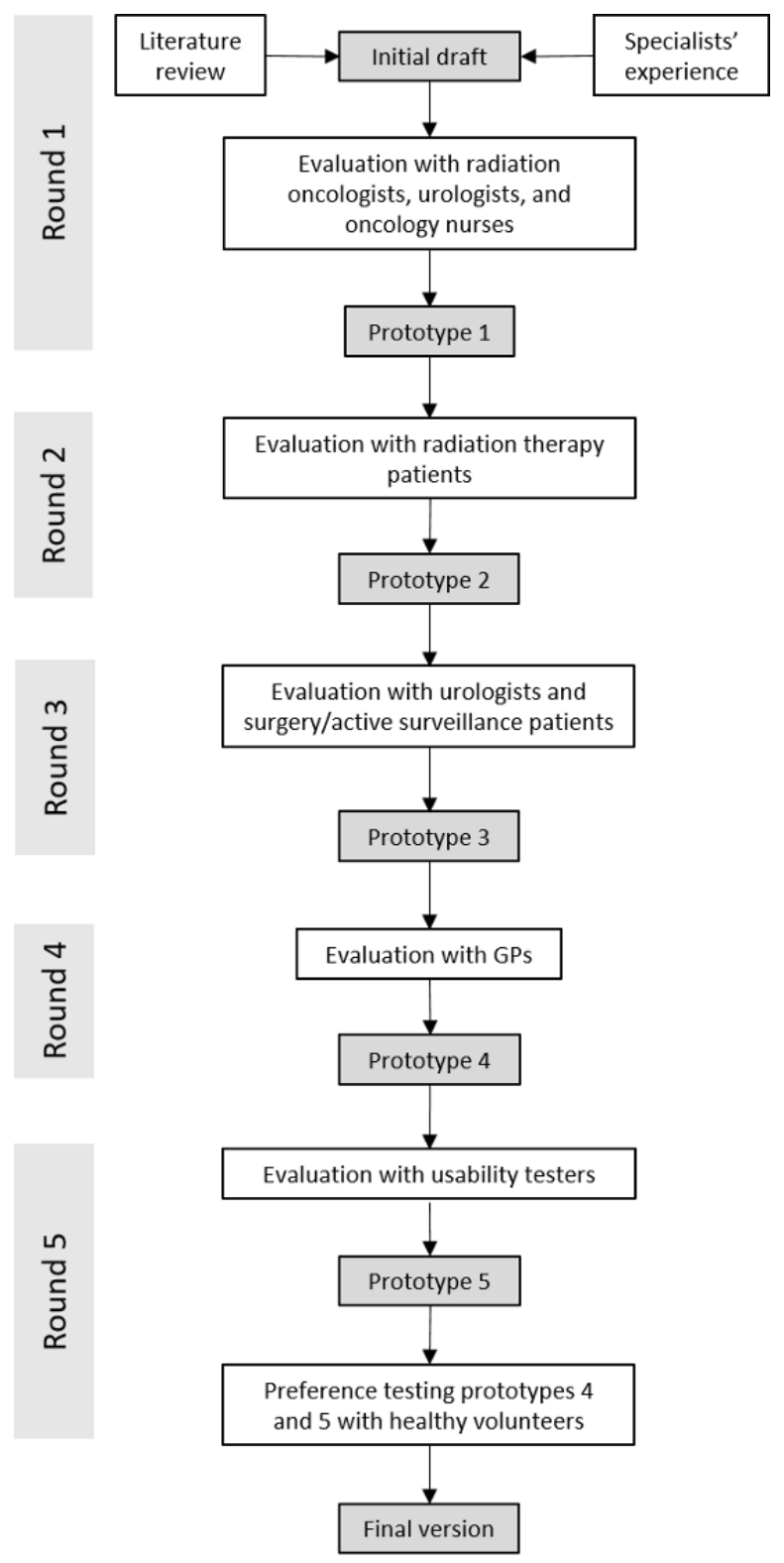

Figure 3.1: A schematic overview of the PDA development process. Each round is evaluated by one or more user groups and produces a prototype that is evaluated in the next round 
Each interview lasted between 30 and $60 \mathrm{~min}$ and was audio-recorded, transcribed, and returned to the respondents for review. After approval, each transcript was analyzed by means of open coding and axial coding [20]. During open coding, a transcript would be divided into text fragments and each fragment would be assigned one or more keywords based on its content. In the subsequent axial coding, the open codes were grouped according to their theme. The final analysis resulted in a list of the most common themes and factors relevant for patients when seeking information and making treatment decisions.

\section{Usability survey}

The respondent was then invited to complete an online 20-item evaluation survey in Dutch based on the unified theory of acceptance and use of technology (UTAUT) [21] in which they scored the comprehensibility and usability of the prototype on a 5-point Likert scale. The survey ended with five open questions asking the respondents to mention positive and negative features of the current prototype and suggestions for improvement. The feedback was used to make incremental adjustments to the prototype in each round. Although not all users filled in the surveys (entirely), we had enough information to improve the existing prototype.

\section{Round 5}

While previous rounds focused on both PDA content and usability with clinicians and patients who had undergone treatment, in Round 5 we focused purely on testing usability and acceptability with nonclinical respondents.

\section{Heuristic evaluation}

Four usability experts evaluated Prototype 4 based on three sets of heuristics and guidelines: general heuristics, readability heuristics, and health-specific usability guidelines. General heuristics measured features such as consistency, informative feedback, appropriate use of error messages, and language use [22,23]. They then used readability heuristics covering topics such as typeface, text size, colors and size 
and style of icons and buttons [24]. Finally, health-specific usability guidelines were based on evidence-based research on health literacy [25]. Their feedback was consolidated into a list of the main usability problems and recommendations, on the basis of which a revised version of the prototype (Prototype 5) was developed.

Preference testing between final two prototypes

Finally, Prototypes 4 and 5 were tested with two groups of 5 and 6 healthy volunteers, respectively, meant to be representative of the target population of PCa patients in terms of age and education level. Volunteers tested the prototypes by means of a think aloud session with the developers. The System Usability Scale (SUS) questionnaire was used to determine the usability of the PDA [26]. The final PDA was based on the strengths of both prototypes.

\subsection{Results}

Clinicians, (former) patients and volunteers were recruited to evaluate the decision aid at different stages in the developmental process. A total of 22 clinicians were consulted, consisting of radiation oncologists, general practitioners, nurses and urologists (Figure 3.2). Their experience and age ranged from older highly experienced staff to younger health care professionals with 2-5 years of experience. Most were familiar with SDM and had basic experience with PDAs. An additional 19 patients and 11 healthy volunteers were consulted. The former patients had received one of the four possible treatment options, which were evenly distributed among the group. The age of patients ranged from 53 to 88 years, with a median age of 73 . The ages of the healthy volunteers ranged from 50 to 90 years, of which $50 \%$ were in the age range 60-69 years. Patients and volunteers were from varying educational backgrounds with an approximately even split between highly educated and minimally educated. This helped to ensure that the content was easy to understand for users from all backgrounds. 
A

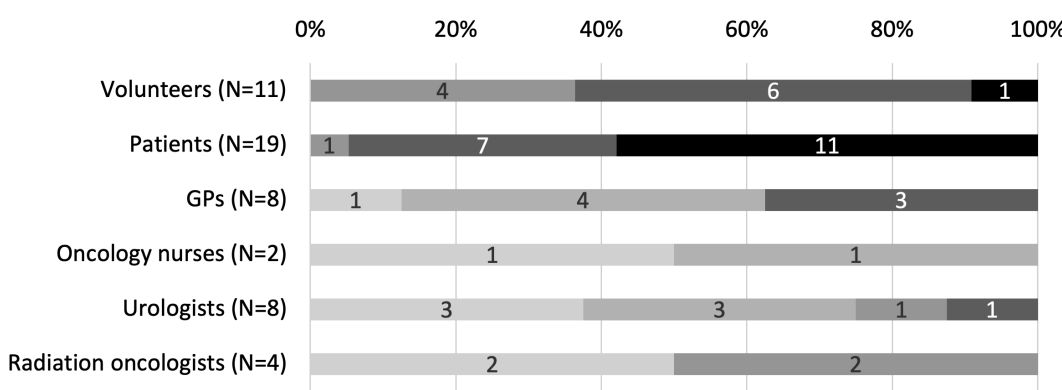

Age (yrs) $\quad 30-39 \square 40-49 \quad \square 50-59 \quad \square 60-69 \quad \square>70$

B

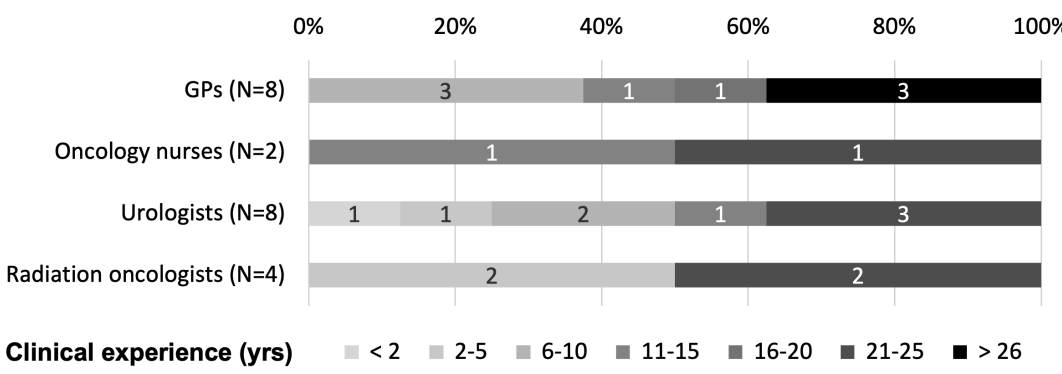

Figure 3.2: The characteristics of the user groups. A) The age distribution of the volunteers, patients, GPs, nurses, urologists and radiation oncologists. B) The clinical experience (years) of all clinicians. The numbers in each bar represent the number of participants in that category

\subsubsection{Round 1}

Clinicians (four radiation oncologists, two urologists and two oncology nurses) mentioned that patients would require a wide range of information to make a well-informed treatment decision, based on most frequently asked questions, which included: anatomy, treatment options, complications per treatment in the short- and long-term and chances of complete cure for each treatment. Additionally, they con- 
sidered it important to structure a PDA in such a way that important information cannot be missed, such as presenting the information in a series of pages that must be clicked through. Information provision would ideally consist of simple jargon-free language and diagrams and videos. The clinicians then evaluated the initial draft (Figure 3.3) which was based on a literature review and clinical experience.
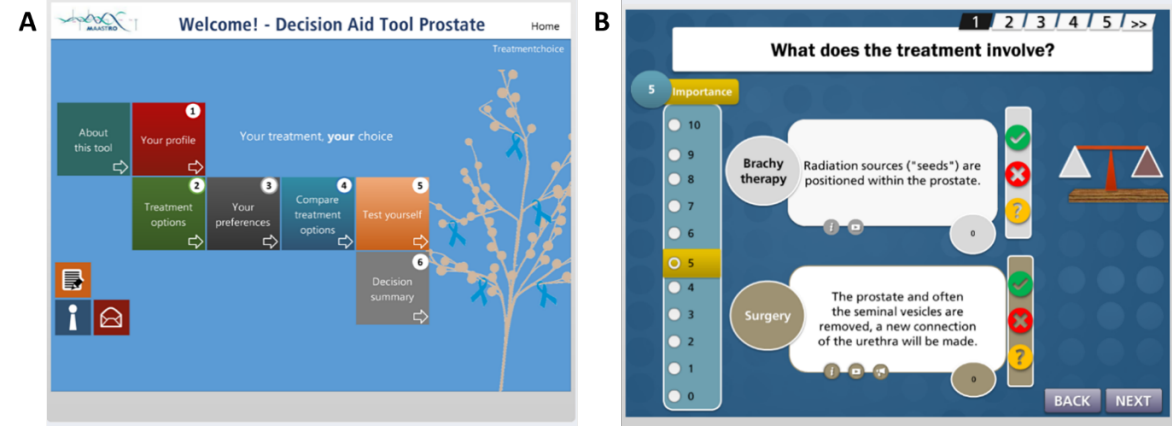

Figure 3.3: Initial draft of the prostate cancer decision aid. A) The proposed welcome screen containing buttons for each section of the tool. B) A proposed layout of the information to be displayed to a patient

The usability survey revealed that clinicians were satisfied with the content and ease of use of the initial draft (Appendix: Figure 3.9), but were concerned about a potential increase in consultation time as a consequence of discussing the patient's preferences more thoroughly due to the PDA (Figure 3.4).

Based on this feedback, the first prototype was developed containing the following sections: (i) a patient profile page in which the patient selects their progression risk level (low, medium, or high); (ii) information about each treatment option based on the risk level selected (for instance, a high risk patient would see information about surgery and radiotherapy whereas a low risk patient would see information about all four treatments); (iii) a series of questions to test their understanding of the presented information and a summary of the results; (iv) a series of questions to identify priorities and preferences; (v) a series 
of questions to identify the least preferable treatment; (vi) a printable summary of the priorities, preferences and answers to the knowledge quiz, and a bar chart displaying the most suitable treatment based on the patient's responses.

$\begin{array}{llllll}0 \% & 20 \% & 40 \% & 60 \% & 80 \% & 100 \%\end{array}$

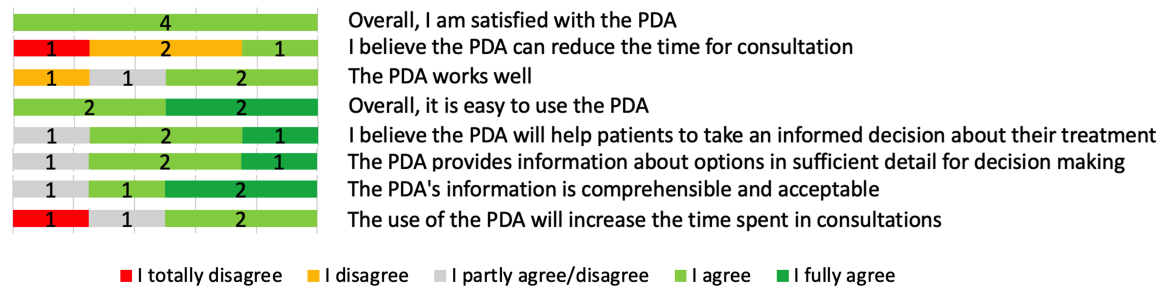

Figure 3.4: An excerpt of the answers to the usability survey questions by the radiation oncologists in round 1 . The numbers in each bar represent the number of participants in that category

\subsubsection{Round 2}

Ten former patients who had undergone radiotherapy were consulted to evaluate prototype 1 . The majority $(80 \%)$ was receptive to using a PDA and felt it would help patients learn more about their condition and ask more focused questions in consultations. The most important factors for decision-making among our respondents was the quality of life after treatment, side effects of the treatment, and the logistical aspects (such as the frequency and duration of hospital visits).

The results of the usability survey indicated that the patients were overall satisfied with the prototype (Appendix: Figure 3.10) but felt that the navigation did not feel intuitive (Figure 3.5). On average patients graded the PDA with an 8.46/10 and needed 43 min to complete the full PDA (this refers to time taken during prototype testing, which included the patient pausing to give feedback to the interviewer. Time taken during non-test settings averaged between 25 and $30 \mathrm{~min}$ ). Based on these comments, prototype 2 was created with the addition 
of: (i) an overview of the pros and cons of all four treatments; (ii) nonclinical questions to the preferences questionnaire.

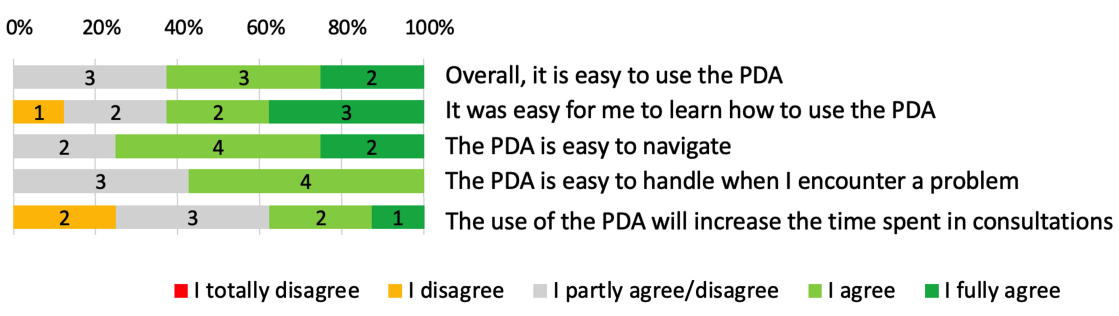

Figure 3.5: An excerpt of the answers to the usability survey questions by the patients in round 2. The numbers in each bar represent the number of participants in that category

\subsubsection{Round 3}

Urologists and patients who had undergone surgery or active surveillance evaluated prototype 2 . The urologists expressed concerns about the difficulties patients have in understanding and remembering clinical information and jargon such as PSA values, Gleason score and TNM-staging. They suggested adding animations to explaining certain treatment procedures. Their reported satisfaction with the PDA was generally neutral and many believed that the PDA would increase patient knowledge and involvement in the decision-making process (Appendix: Figure 3.11). However, only 50\% would recommend the PDA and $83 \%$ were not satisfied with how the PDA worked and the information that was presented (Figure 3.6). On the other hand, patients felt that aspects of treatment such as side effects and the treatment trajectory were not adequately covered in the consultations. Still, the majority was positive about the comprehensibility and usability of the prototype (Appendix: Figure 3.12). In fact, they scored the PDA with an 8.56 on a scale of $1-10$ and required an average of $54 \mathrm{~min}$ to complete the PDA. Additionally, patients were overall more satisfied with the PDA than the clinicians. 

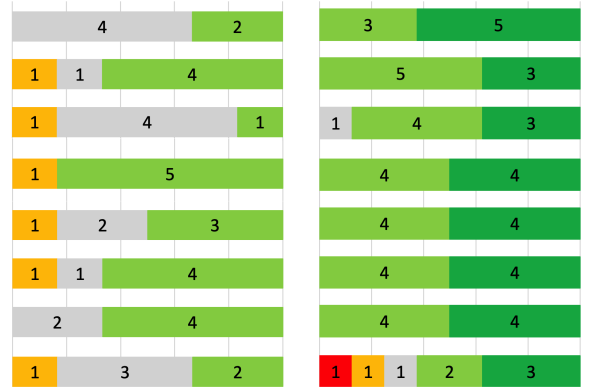

Overall, I am satisfied with the PDA

The PDA can improve patient care

The PDA works well

The PDA will help with taking an informed decision

I would recommend the PDA to others

If the PDA is available to me I will definitely use it

The PDA's information is comprehensible and acceptable

Using the PDA will increase consultation time

a totally disagree $\quad$ I disagree $\quad$ I partly agree/disagree $\quad$ I agree $\quad$ I fully agree

Figure 3.6: An excerpt of the questions and corresponding answers in the usability survey. The left column contains the answers given by the clinicians (urologists) and the right column provides the results of the questionnaire from the patients

Based on this input, prototype 3 was developed, in which (i) the profile section was simplified by removing jargon; (ii) videos were added for each treatment procedure; (iii) a statement was added to emphasize that the treatment options all yield similar survival chances.

\subsubsection{Round 4}

Eight GPs were interviewed on patients' decisional needs and to evaluate prototype 3 . They observed that patients generally chose aggressive treatments such as surgery hoping to eradicate the tumor completely, without considering the impact of the side effects, and were heavily influenced by experiences of former patients and friends and the opinion of the urologist treating them.

Of the 8 GPs, 5 filled in the usability survey and were generally satisfied with the prototype (usability, navigation, content and potential to support SDM; Appendix: Figure 3.13) but felt that its use would not reduce the consultation time (Figure 3.7). 


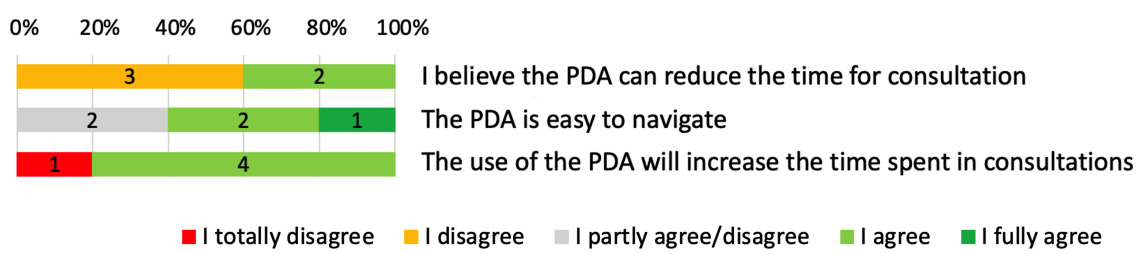

Figure 3.7: An excerpt of the questions and corresponding answers in the usability survey as answered by 5 of the 8 GPs

In response to this feedback, the following changes were made in prototype 4: (i) the bar chart showing the treatment recommendation was replaced with an overview of all treatment options and their relative importance to the patient; (ii) questions regarding side effects were clarified to improve understanding.

\subsubsection{Round 5}

Usability experts heuristically evaluated prototype 4 and revealed problems regarding navigation, visual presentation and readability. For instance, navigation inconsistencies could lead users to miss information without realizing it. Additionally, they advised against visually distracting elements such as bright colors, background images, and cluttered text with small font sizes. These issues were incorporated into Prototype 5 and both prototypes (4 and 5) were presented to volunteers for testing. SUS scores for Prototypes 4 and 5 were 79 (standard deviation $=10.09$ ) and 84.6 (standard deviation $=18.4$ ) respectively, however a t-test revealed that the difference was not statistically significant. $78 \%$ of volunteers preferred the closed navigation structure of Prototype 5. There was little consensus regarding layout, with an even split in preference between Prototype 4 and 5. Overall, the participants preferred the simpler visuals of Prototype 5. 


\subsubsection{Final PDA Design}

Based on the results of the interviews and usability feedback, the final version of the PDA in Dutch language, which is freely available on https://beslissamen.nl/, contains seven sections:

1. Introduction, where a 2-min video introduces the purpose of the PDA and its contents.

2. Personal treatment options, where patients provide personal information (e.g. name, date of birth, disease characteristics) on which the information provision is risk-stratified. For instance, a low risk patient will see information about brachytherapy (as displayed in Figure 3.8A).

3. A side-by-side comparison of the relevant pros and cons of the applicable treatment options.

4. A knowledge quiz consisting of 10 true/false questions to help patients assess whether they have understood the provided information sufficiently.

5. A questionnaire containing 16 questions to clarify a patient's preferences and priorities regarding treatment experience, quality of life, and uncertainty (Figure 3.8B).

6. Questions relating to treatment experiences to indicate the least desirable option.

7. A summary of the patient's preferences and priorities in relation to each treatment option, including the option to print the summary and a selection of questions and notes, which can be used during the next consultation. 

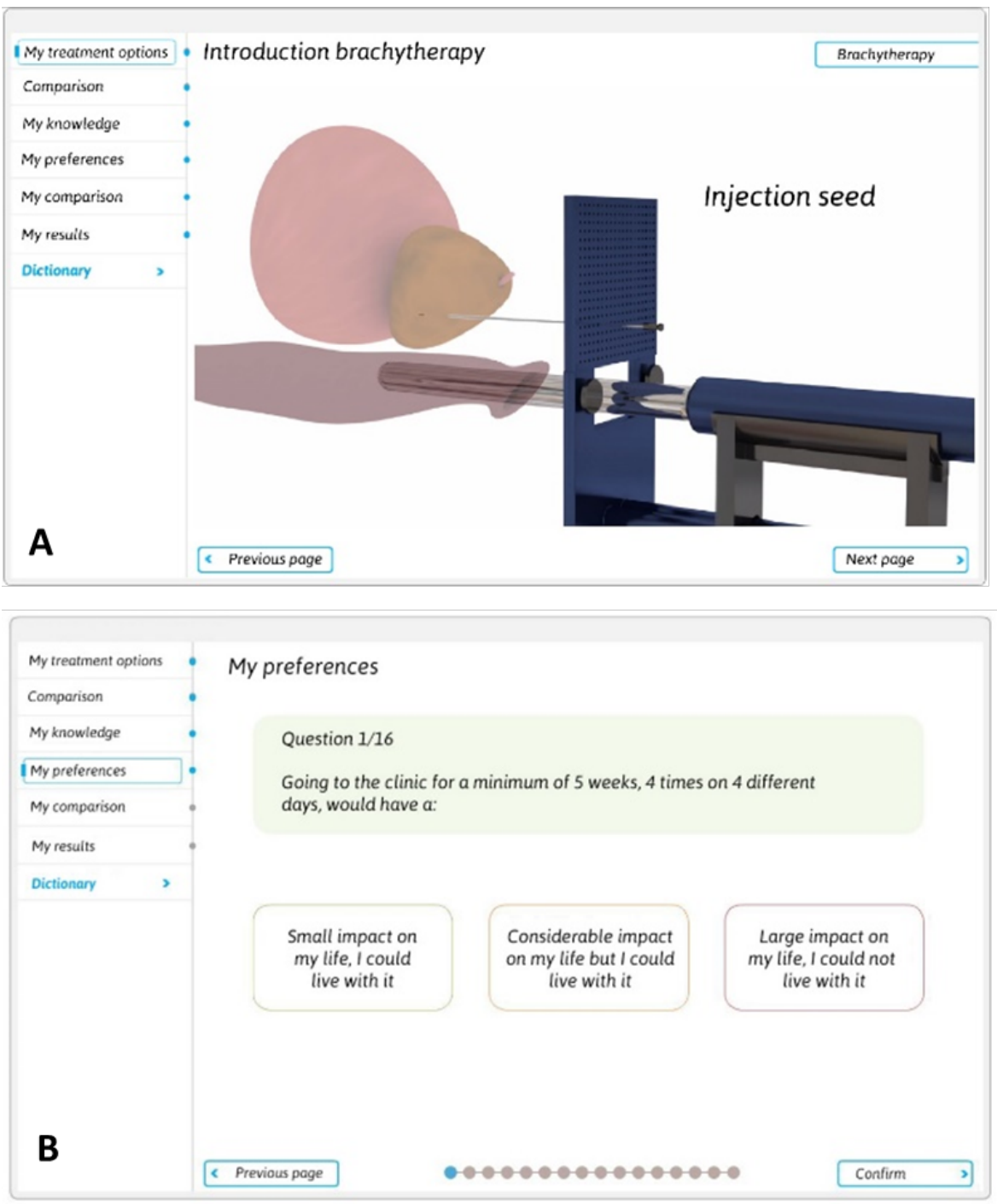

Figure 3.8: Screenshots of the final PDA version. Textual information was translated from Dutch to English for this Figure. A) A screenshot of one of the pages introducing brachytherapy in the PDA. On this page, an animation coupled with a voice-over explains the procedure in an understandable way. B) A screenshot of one of the preferences questions and its potential answers 


\subsection{Discussion}

Based on IPDAS guidelines and a user-centered design framework, we developed a freely accessible web-based PDA for PCa in Dutch. Clinicians, patients, volunteers and usability experts were consulted to provide feedback on the content of the PDA and evaluate its usability at every stage of development. Patients stressed the importance of specific details of each procedure, such as short- and long-term side effects, post-treatment care and (side-by-side) pros and cons of each treatment. On the other hand, clinicians mentioned the importance of clinically relevant information. Our findings are consistent with previous findings that clinicians and patients evaluate treatment options differently: clinicians tend to focus on clinical outcomes, whereas patients also consider how the treatment will affect their (quality of) life on the short- and long-term [27]. To consolidate these different views, a common platform is needed to make an informed decision based on both perspectives. During the development of our PDA, this meant moving the focus away from clinical aspects of the disease and toward its effect on the patient's daily life.

The main concern among clinicians was that a PDA would increase the consultation time, which has been cited as one of the major barriers in the implementation of SDM and PDAs [28, 29]. Although our PDA is intended to be used at home by the patient rather than in the clinical setting, thus not directly interfering with consultation times, the aim is to inform patients well enough that they can engage with clinicians to thoroughly discuss their treatment options. Naturally, this may lead to longer consultation times. There is inconclusive evidence on the effect of PDAs on consultation length; some studies found that consultations are on average longer while others found reduced times, with the median change being an increase of $2.6 \mathrm{~min}$ [5]. Prior findings also suggest that the initial costs of using a PDA might be offset if it results in the patient being better informed during the consultation, particularly if it results in a reduction in overtreatment [30]. Nevertheless, clinicians' views about PDAs adding to their workflows remain a valid concern. 
One possible solution is to present the PDA output in a format which supports the shared decision talk. For instance, in our PDA the patient's answers are summarized in a printable tabular format: all relevant treatment options and their side effects are shown with dots representing their relative impact on the patient's life. For instance, a strong preference for avoiding erection problems is indicated with three dots whereas if the patient is not concerned about long hospital stays this is indicated with one dot. The patient and clinician can see then see his preferences at a glance and zero in on the most important concerns, thus going through the consultation more efficiently.

Another concern expressed by clinicians was that the advanced age of most PCa patients may hamper their use of web-based PDAs. A systematic review of PDA effectiveness in elderly populations concludes that elderly patients who use PDAs display better knowledge and risk perception and lower decisional conflict [31]. The reviewers concede however that little attention has been paid to this topic in the literature and that few PDAs are specifically designed for elderly patients. Moreover, there is little guidance on how to adapt PDAs for an older audience, leading the reviewers to emphasize the importance of proper design and testing. Visual support in the form of diagrams [32] and animations [33] is considered particularly beneficial. The involvement of elderly patients under user-centered design may be a crucial step in understanding how to present complex clinical information to an older audience.

Our process brought to light some of the strengths and pitfalls of usercentered design. The iterative model may make it easier for developers to adapt data gathering and testing methods from initial rounds to new treatment information and make incremental changes as required. However, one of the drawbacks is that involving a wide group of stakeholders in an iterative process can make the development process long, complicated and costly. Our development process spanned over two years and involved 58 participants, resulting in over 100 hours of interview material and feedback that needed to be processed, analyzed and incorporated in successive rounds. Although it is beneficial to the 
quality of the content and usability, developers should take into account the resources required to create a high quality PDA.

Additionally, engaging many different users provides a variety of perspectives, but can pose a larger challenge to integrate these diverse views and find a consensus, particularly in the area of usability testing. For instance, some participants found the PDA's closed navigation essential to guide the user through the process while others felt it was too restrictive. Incorporating these varying perspectives and preferences may not be feasible, so at times developers may need to consult evidence-based guidelines or establish consensus through methods such as focus groups or a Delphi study [34].

Aside from development, it is crucial to ensure that maintenance is provided both technologically and scientifically. For instance, making tablet-ready applications is becoming a necessity as more users are transitioning from laptops and desktop computers to tablets and smartphones for their daily browsing [35]. Additionally, (prostate) cancer treatment is a rapidly developing field with the emergence of new modalities like proton therapy. It is important to ensure that a PDA stays up-to-date on current developments and new therapies, which requires developers and clinicians to collaborate continuously. Therefore, it may be valuable to establish a governance structure within the hospital for maintaining the PDA and collecting and analyzing data about its effectiveness to be shared with clinicians and the wider medical community to create awareness.

Future work may explore more detailed value clarification methodologies to identify additional factors that influence the use of PDAs in clinical practice [36]. Additionally, translations to other languages would greatly benefit the non-Dutch patient population inside and outside the Netherlands. Furthermore, a key future step will be the personalization of our PDA by incorporating data from prediction models (www.predictcancer.org) for survival, toxicity or side effects to encourage further personalized shared decision making $[6,37]$. Data from individual hospitals could be incorporated as well to tailor the PDA to 
a specific hospital and create ownership. It is important to note that tools such as these would likely require certification such as CE marking [38].

Our process and the resulting PDA is currently validated in a clinical trial (NCT03278197, ProDecA, Evaluation of a Web-based Decision Aid Tool for PCa Patients), where the effect of the PDA in clinical practice will be evaluated. We expect to observe improvements in patient knowledge and the SDM process and a decrease in decisional conflict.

\subsection{Conclusion}

In this study we followed a user-centered design process to develop a web-based PDA for PCa patients. The development process spanned five rounds over a period of two years and involved both former PCa patients and health-care professionals. This process resulted in a PDA that meets the standards of both clinicians and patients by presenting balanced information about the four available treatment options and by providing a platform for patients to explore their personal preferences and priorities. 


\section{Appendix}

\section{$\begin{array}{llllll}0 \% & 20 \% & 40 \% & 60 \% & 80 \% & 100 \%\end{array}$}

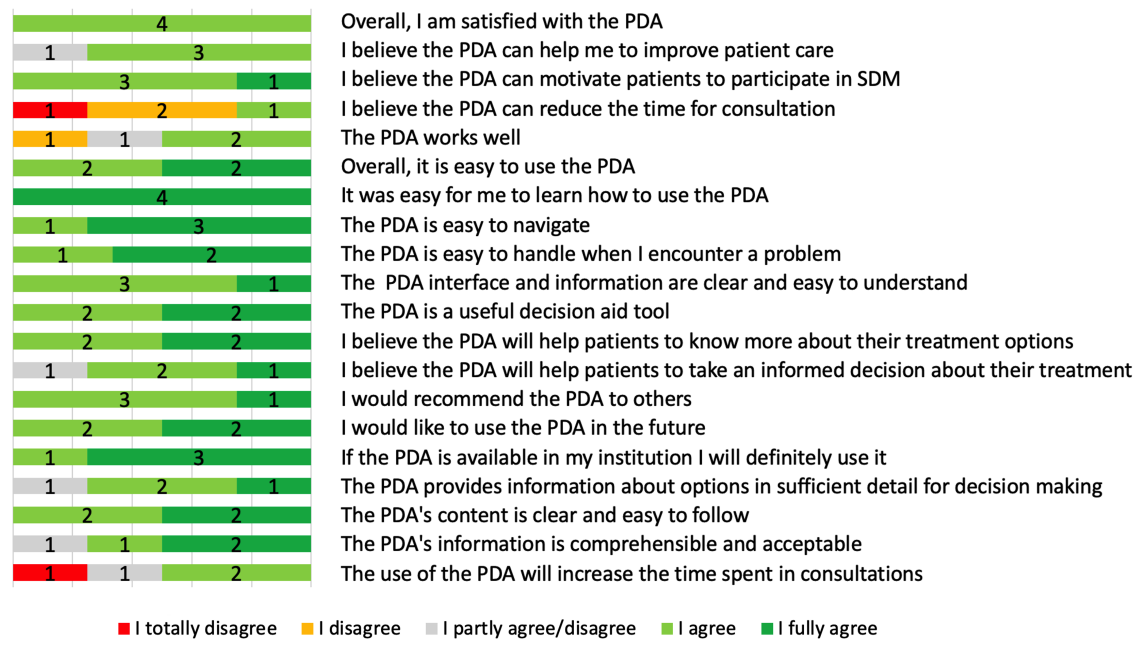

Figure 3.9: The complete list of questions and corresponding answers of the usability survey filled in by the radiation oncologists in round 1 . 


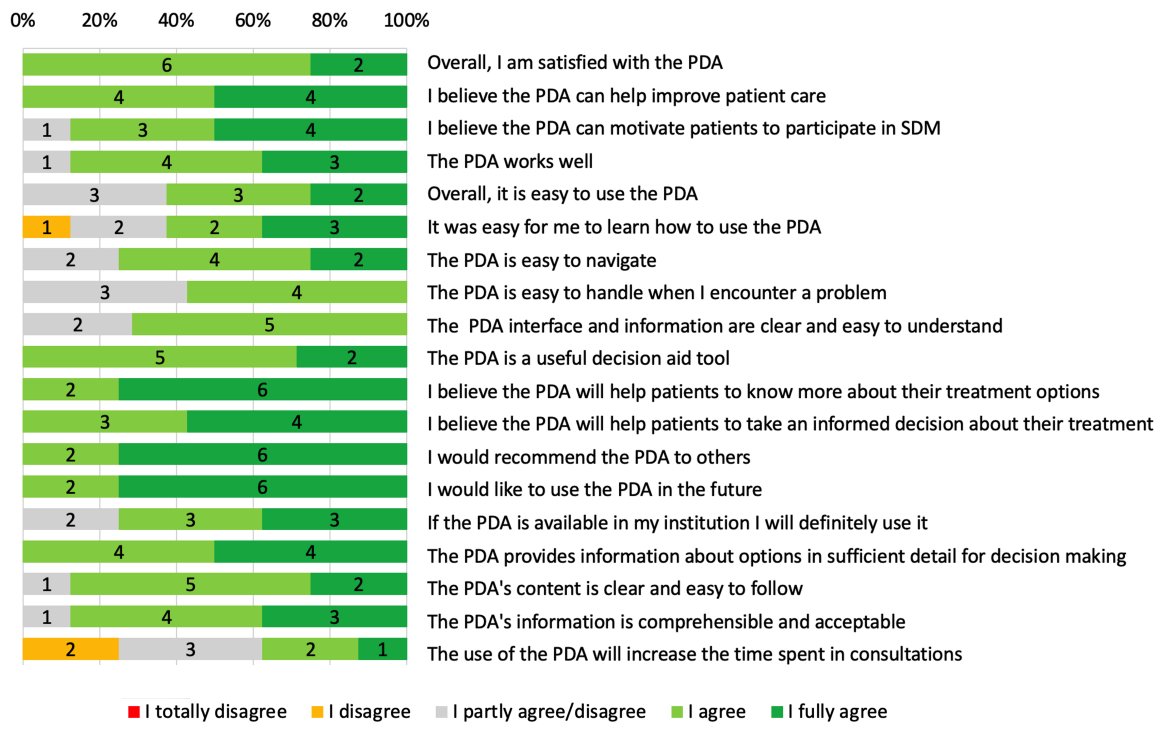

Figure 3.10: The complete list of questions and corresponding answers of the usability survey filled in by the radiotherapy patients in round 2 . 


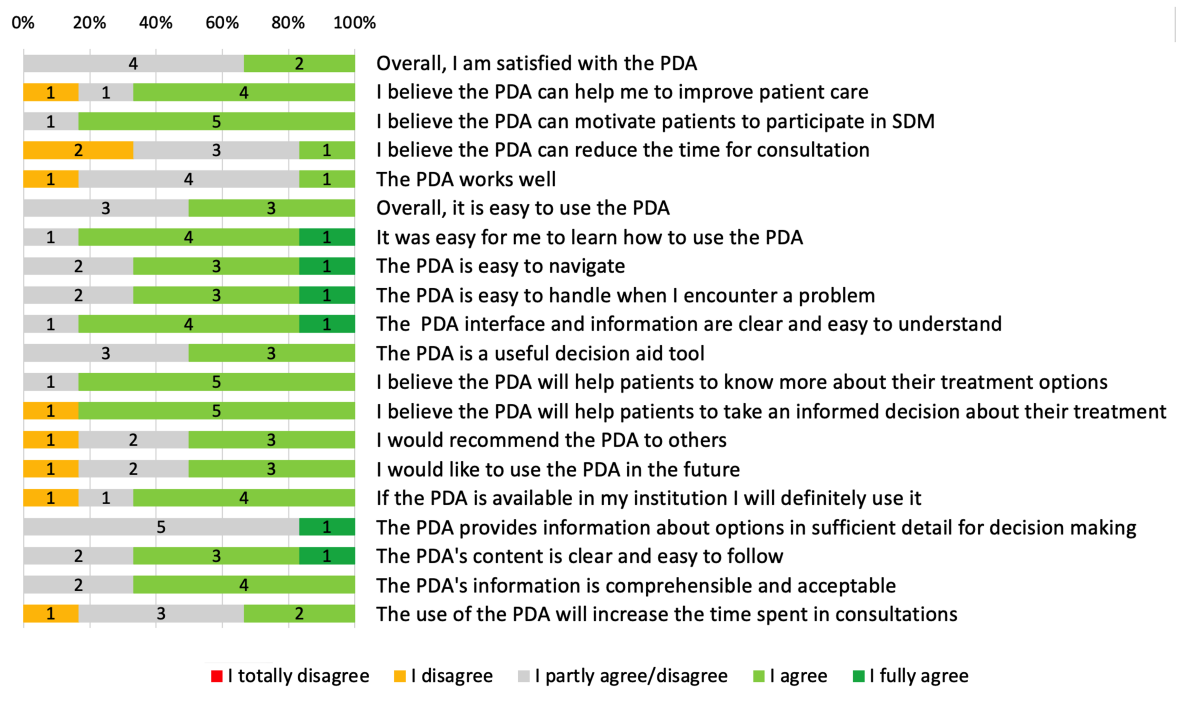

Figure 3.11: The complete list of questions and corresponding answers of the usability survey filled in by the urologists in round 3 . 


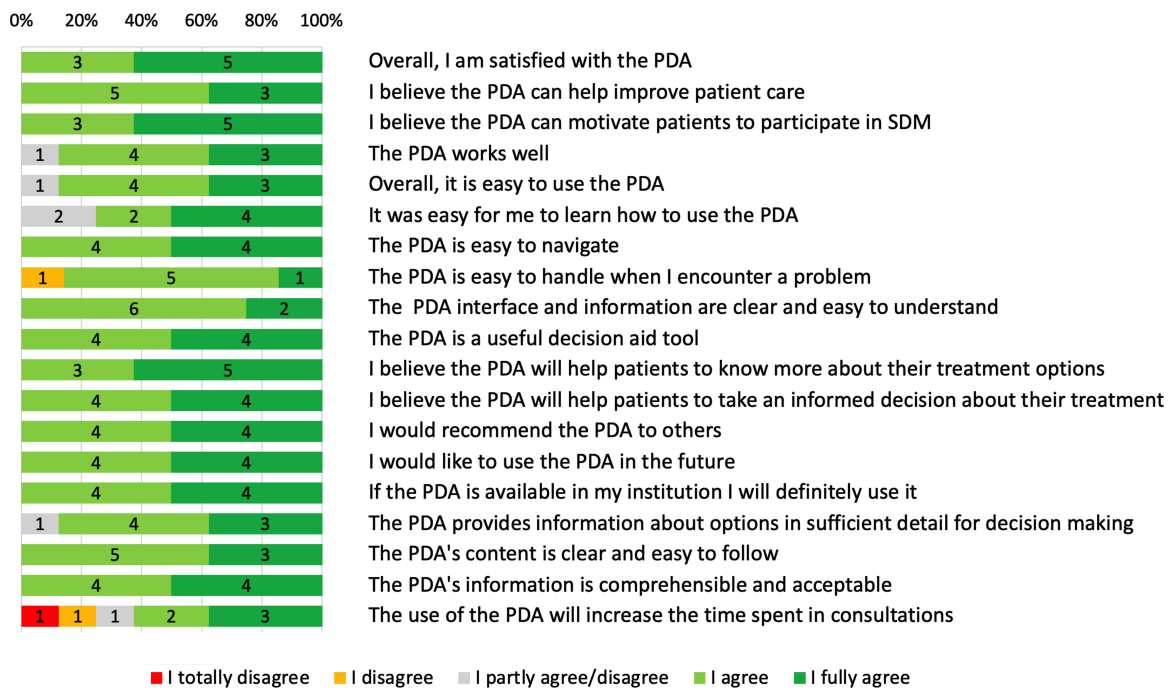

Figure 3.12: The complete list of questions and corresponding answers of the usability survey filled in by the surgery and active surveillance patients in round 3 . 


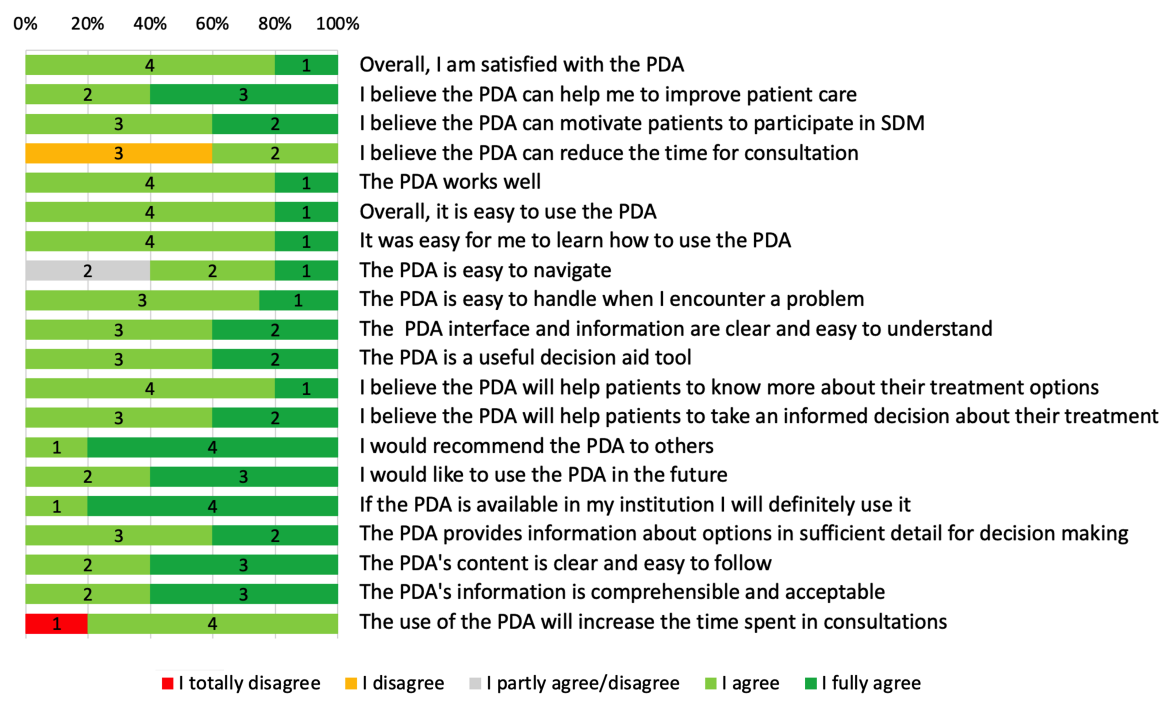

Figure 3.13: The complete list of questions and corresponding answers of the usability survey filled in by the general practitioners (GPs) in round 4. 


\section{References}

1. Bray F, Ferlay J, Soerjomataram I, Siegel RL, Torre LA, Jemal A. Global cancer statistics 2018: GLOBOCAN estimates of incidence and mortality worldwide for 36 cancers in 185 countries. CA Cancer J Clin. 2018;68(6):394-424.

2. Pishgar F, Ebrahimi H, Moghaddam SS, Fitzmaurice C, Amini E. Global, regional and national burden of prostate cancer, 1990 to 2015: Results from the global burden of disease study 2015. J Urol. 2018;199(5):1224-32.

3. Vanneste BG, Van Limbergen EJ, van Lin EN, van Roermund JG, Lambin P. Prostate Cancer radiation therapy: what do clinicians have to know? Biomed Res Int. 2016;1(2016):1-14.

4. Charles C, Gafni A, Whelan T. Shared decision-making in the medical encounter: what does it mean?(or it takes at least two to tango). Soc Sci Med. 1997;44(5):681-92.

5. Stacey D, Légaré F, Lewis K, Barry MJ, Bennett CL, Eden KB, et al. Decision aids for people facing health treatment or screening decisions. Cochrane Libr. 2017;4:1-297.

6. Lambin P, Zindler J, Vanneste BG, Van De Voorde L, Eekers D, Compter I, et al. Decision support systems for personalized and participative radiation oncology. Adv Drug Deliv Rev. 2017;109:131-53.

7. Lamers RE, Cuypers M, Husson O, Vries M, Kil PJ, Ruud Bosch J, et al. Patients are dissatisfied with information provision: perceived information provision and quality of life in prostate cancer patients. Psycho-Oncology. 2016;6:633-40.

8. Zafar SY, Alexander SC, Weinfurt KP, Schulman KA, Abernethy AP. Decision making and quality of life in the treatment of cancer: a review. Support Care Cancer. 2009;17(2):117-27.

9. Davison BJ, So AI, Goldenberg SL. Quality of life, sexual function and decisional regret at 1 year after surgical treatment for localized prostate cancer. BJU Int. 2007;100(4):780-5.

10. Sciarra A, Gentile V, Panebianco V. Multidisciplinary management of prostate Cancer: how and why. Am J Clin Exp Urol. 2013;1(1):12.

11. Hamdy FC, Donovan JL, Lane JA, Mason M, Metcalfe C, Holding P, et al. 10-year outcomes after monitoring, surgery, or radiotherapy for localized 
prostate cancer. N Engl J Med. 2016;375(15):1415-24.

12. Donovan JL, Hamdy FC, Lane JA, Mason M, Metcalfe C, Walsh E, et al. Patient-reported outcomes after monitoring, surgery, or radiotherapy for prostate cancer. N Engl J Med. 2016;375(15):1425-37.

13. Oshima Lee E, Emanuel EJ. Shared decision making to improve care and reduce costs. N Engl J Med. 2013;368(1):6-8.

14. O'Connor A. Using patient decision aids to promote evidence-based decision making. ACP J Club. 2001;135(1):A11-A.

15. Elwyn G, Scholl I, Tietbohl C, Mann M, Edwards AG, Clay C, et al. "Many miles to go...": a systematic review of the implementation of patient decision support interventions into routine clinical practice. BMC Medical Informatics and Decision Making. 2013;13(2):1.

16. Witteman HO, Dansokho SC, Colquhoun H, Coulter A, Dugas M, Fagerlin A, et al. User-centered design and the development of patient decision aids: protocol for a systematic review. Systematic reviews. 2015;4(1):11.

17. Abras C, Maloney-Krichmar D, Preece J. User-centered design. Bainbridge, W Encyclopedia of Human-Computer Interaction Thousand Oaks: Sage Publications. 2004;37(4):445-56.

18. Noone A, Howlader N, Krapcho M, Miller D, Brest A, Yu M, et al. SEER Cancer statistics review, 1975-2015. Bethesda: National Cancer Institute; 2018.

19. Elwyn G, O'Connor A, Stacey D, Volk R, Edwards AG, Coulter A, et al. International patient decision aids standards (IPDAS) collaboration. Developing a quality criteria framework for patient decision aid: online international Delphi consensus process. Br Med J. 2006;333(7565):417-9.

20. Strauss A, Corbin J. Basics of qualitative research. Los Angeles: Sage publications; 1990.

21. Venkatesh V, Morris MG, Davis GB, Davis FD. User acceptance of information technology: toward a unified view. MIS Q. 2003:425-78.

22. Hesse BW, Shneiderman B. eHealth research from the user's perspective. Am J Prev Med. 2007;32(5):S97-S103.

23. Nielsen J. Usability engineering. Cambridge: Elsevier; 1994. 
24. Hodes RJ, Lindberg DA. Making your website senior friendly. Bethesda: National Institute on Aging and the National Library of medicine; 2002.

25. Monkman H, Griffith J, Kushniruk AW, editors. Evidence-based heuristics for evaluating demands on eHealth literacy and usability in a Mobile consumer health application. Stud Health Technol Inform. 2015;216:358-62.

26. Brooke J. SUS-A quick and dirty usability scale. Usability evaluation in industry. 1996;189(194):4-7.

27. Lee CN, Dominik R, Levin CA, Barry MJ, Cosenza C, O'Connor AM, et al. Development of instruments to measure the quality of breast cancer treatment decisions. Health Expect. 2010;13(3):258-72.

28. Bekker HL, Hewison J, Thornton JG. Applying decision analysis to facilitate informed decision making about prenatal diagnosis for Down syndrome: a randomised controlled trial. Prenat Diagn. 2004;24(4):265-75.

29. Légaré F, Ratté S, Gravel K, Graham ID. Barriers and facilitators to implementing shared decision-making in clinical practice: update of a systematic review of health professionals' perceptions. Patient Educ Couns. 2008;73(3):526-35.

30. Knops AM, Legemate DA, Goossens A, Bossuyt PM, Ubbink DT. Decision aids for patients facing a surgical treatment decision: a systematic review and meta-analysis. Ann Surg. 2013;257(5):860-6.

31. Van Weert JC, Van Munster BC, Sanders R, Spijker R, Hooft L, Jansen J. Decision aids to help older people make health decisions: a systematic review and meta-analysis. BMC medical informatics and decision making. 2016;16(1):45.

32. Bol N, van Weert JC, de Haes HC, Loos EF, de Heer S, Sikkel D, et al. Using cognitive and affective illustrations to enhance older adults' website satisfaction and recall of online cancer-related information. Health Commun. 2014;29(7):678-88.

33. Meppelink CS, van Weert JC, Haven CJ, Smit EG. The effectiveness of health animations in audiences with different health literacy levels: an experimental study. J Med Internet Res. 2015;17(1):e11.

34. Hsu C-C, Sandford BA. The Delphi technique: making sense of consensus. Practical assessment, research \& evaluation, vol. 12; 2007. p. 10):1-8. 
35. Székely A, Talanow R, Bágyi P. Smartphones, tablets and mobile applications for radiology. Eur J Radiol. 2013;82(5):829-36.

36. Fagerlin A, Pignone M, Abhyankar P, Col N, Feldman-Stewart D, Gavaruzzi T, et al. Clarifying values: an updated review. BMC medical informatics and decision making. 2013;13(2):S8.

37. Lambin P, Van Stiphout RG, Starmans MH, Rios-Velazquez E, Nalbantov G, Aerts HJ, et al. Predicting outcomes in radiation oncology-multifactorial decision support systems. Nat Rev Clin Oncol. 2013;10(1):27-40.

38. Guidelines on the qualification and classification of stand alone software used in healthcare within the regulatory framework of medical devices, (2016). 



\section{4}

\section{Implementing shared decision-making in prostate cancer: Insights from primary and secondary care}

Ankolekar, A., Vanneste, B., Bloemen-van Gurp, E., Van Roermund, J., Berlanga, A., Roumen, C., Van Limbergen, E., Lutgens, L., Marcelissen, T., Lambin, P., Dekker, A., \& Fijten, R.

Submitted 


\section{Abstract}

\section{Background}

Increasingly, patients wish to participate in their treatment decisions through shared decision-making (SDM), however clinicians cite practical and patient-related barriers. Since clinicians guide the decisionmaking process, it is vital to understand their perspective so that patient preferences can be incorporated in treatment decisions. The goal of this qualitative study is to define an implementation strategy for SDM and a prostate cancer patient decision aid (PDA) to support patient engagement.

\section{Methods}

Seventeen semi-structured interviews were conducted with GPs $(n=8)$ and prostate cancer specialists $(\mathrm{n}=9)$, focusing on the decision-making process, perceptions about patient participation, SDM implementation barriers, and facilitators. Transcripts were analyzed through open and axial coding.

\section{Results}

Seven themes emerged: (1) patients' participation preference/ability, (2) information provision, (3) trust, (4) influence of multiple sources, (5) time, (6) SDM as a burden, and (7) the treatment trajectory. GPs emphasized patients' need for trust and reassurance, while specialists suggested logistical factors that may support patient participation.

\section{Discussion}

Successful patient engagement requires better information provision and a trusting relationship. GPs and specialists can use their complementary skills and resources to support patients in both these areas. This can be achieved through three structural facilitators: (1) a greater role for GPs, (2) embedding PDAs in the care pathway, and (3) multidisciplinary clinics. We contrast the current care pathway with a proposed care pathway that incorporates these three facilitators based on our findings. 
Conclusion

Efforts to implement SDM must take into account the distributed nature of the decision-making process and adjust the care pathway accordingly. 



\subsection{Introduction}

Men diagnosed with prostate cancer (PCa) face a choice between several treatment options (e.g., radical prostatectomy, external beam radiation, brachytherapy, active surveillance) with comparable survival rates but different side-effects [1]. Since there is often no single best treatment, the choice comes down to the patient's preference. However, patients vary in their ability to articulate their preferences during consultations and often report unmet expectations resulting in decisional regret [2].

Shared decision-making (SDM) aims to align treatment decisions with patient preferences through collaborative two-way communication and information exchange between patients and clinicians [3]. The majority of patients are enthusiastic about SDM [4], however clinicians tend towards skepticism [5], citing barriers such as time constraints and lack of applicability due to the patient/clinical situation [6]. Suboptimal communication between patients and clinicians represents another barrier [7].

SDM can be supported by patient decision aids (PDAs), tools that inform patients about their disease and treatment options and help them clarify their preferences [8]. Unfortunately, many PDAs fail to be implemented in practice [9]. Furthermore, SDM is often conflated with PDAs [10], even though PDAs do not guarantee that SDM will take place, and may not improve outcomes if the clinic environment is not conducive to SDM. Since successful implementation of health innovations relies on the input, cooperation, and enthusiasm of clinicians, it is imperative to study their perspective across the treatment trajectory.

In this study, we investigate Dutch general practitioners' (GPs) and PCa specialists' perspectives about factors that affect PCa patients' ability to engage in SDM. As primary care providers, GPs have a closer/longer-term relationship with patients and are suited to comment on the patient experience of PCa treatment. In addition, GPs play a key role in supporting patients from diagnosis to post-treatment care [11]. Specialists' perspectives were considered 
essential as they conduct the decision-making process with the patient. This study was conducted as a precursor to implementing a web-based PDA for PCa developed at our clinic with a user-centered design [12].

\subsection{Methods}

\subsubsection{Study Design}

In this qualitative study, we performed semi-structured interviews with GPs, who represented the patient perspective, and PCa specialists, who represented the clinical perspective. This format was chosen so that participants could provide in-depth information about their experiences and concerns regarding patient participation through SDM. Three researchers constructed interview guides (provided in the appendix) based on a literature review of barriers and facilitators to SDM in general [6], SDM implementation in PCa specifically, and the International Patient Decision Aids Standards (IPDAS). The interview guide covered the following areas: the current PCa trajectory, awareness of SDM principles, attitudes towards SDM and PDAs, perceived factors that might inhibit patient engagement in SDM, and organizational actions that can foster SDM.

MAASTRO's Internal Review Board (IRB) reviewed and approved this study.

\subsubsection{Participants}

GPs were contacted through the MUMC+ Department of Family Medicine with the main inclusion criterion being that they were active in South Limburg, as most PCa patients at our clinic are referred within this region. Specialists were recruited through the MUMC+ Oncology Center and the radiotherapy department (MAASTRO) via purposive sampling. Inclusion criteria were as follows: involvement in PCa treatment decisions with patients or supporting patients 
making these decisions; knowledge and/or basic experience with SDM and PDAs. As the aim was to get a range of perspectives, urologists, radiation oncologists, and oncology nurses were invited to participate and there was no minimum requirement regarding years of clinical experience.

\subsubsection{Data Collection and Analysis}

All interviews were conducted at the participant's workplace. Written informed consent was obtained from all participants beforehand. Interviews lasted 30-60 minutes and were audiotaped. The recordings were transcribed fully, anonymized, and emailed back to the participants for clarification and approval. Each transcript was analyzed by means of open coding, in which text fragments were assigned codes summarizing their meaning. Subsequently, we performed axial coding, in which the open codes were grouped according to their underlying themes. To improve validity all transcripts were coded independently by two researchers and a third researcher oversaw the coding process and analysis. Discrepancies were discussed until consensus was reached.

\subsection{Results}

Seventeen participants (eight GPs and nine PCa specialists) agreed to be interviewed (Table 4.1). GPs (age range, 35-65 years) had clinical experience ranging from six years to over 25 years. Specialists (age range, 33-58 years) included four urologists, three radiation oncologists, and two oncology nurses specialized in urology, with experience ranging from 3 to 20 years. Most participants had knowledge about SDM as a concept, but none had formal experience of its application in their clinics, such as through training or other implementation efforts.

Our analysis brought to light seven factors that participants felt affect patient engagement in SDM, of which two were common between 
Table 4.1: Demographic characteristics of interview participants

\begin{tabular}{lcc}
\hline & $\begin{array}{c}\text { GPs } \\
(\mathbf{n}=8)\end{array}$ & $\begin{array}{c}\text { Specialists } \\
(\mathbf{n}=9)\end{array}$ \\
\hline Age (yrs) & 1 & 4 \\
$30-39$ & 4 & 2 \\
$40-49$ & & 3 \\
$50-59$ & 3 & \\
$60-69$ & & \\
$70+$ & & 6 \\
Gender & 6 & 3 \\
Male & 2 & 3 \\
Female & & 4 \\
Specialization & & 2 \\
Radiation oncologist & & \\
Urologist & & 3 \\
Oncology nurse & & 1 \\
Experience (yrs) & & 1 \\
$2-5$ & & 4 \\
6-10 & 3 & \\
11-15 & 1 & \\
16-20 & 1 & \\
$21-25$ & 3 & \\
$25+$ & &
\end{tabular}

GPs and specialists: 'patients' participation preference and ability' and 'information provision'. The remaining factors were unique to GPs ('trust' and 'influence of multiple sources') and specialists ('time', 'SDM as a burden', and 'treatment trajectory'). An overview of the themes is provided in Figure 4.1 and sample quotes from participants are presented in Table 4.2.

Although over half the specialists felt that patients are willing to take part in decision-making, several factors were cited that may hinder their participation. Three specialists felt that elderly patients are still used to paternalistic decision-making and may find SDM challenging. Differences in educational backgrounds and ability to understand clin- 


\section{GPs}

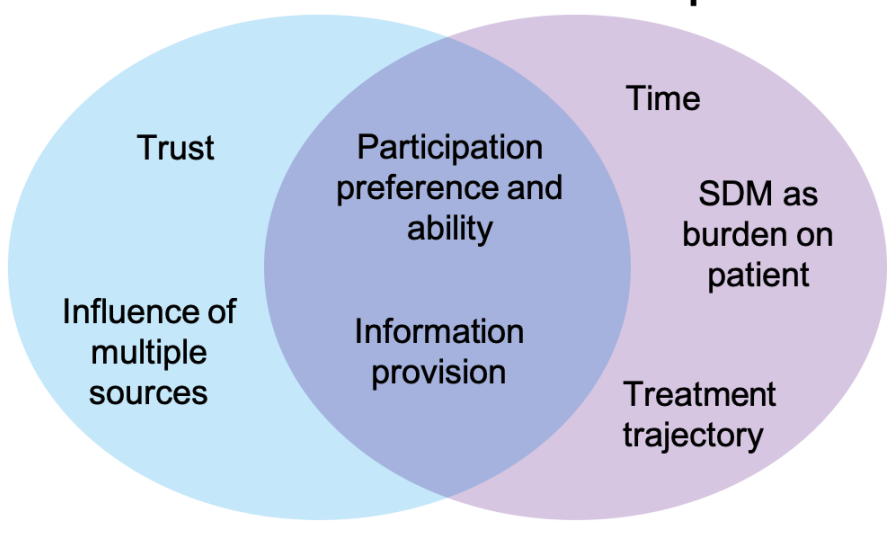

Figure 4.1: Factors that affect PCa patients' participation in decision-making, according to GPs and specialists.

ical information presented another challenge according to one specialist.

These sentiments were partially reflected in GPs' responses. Over half felt that PCa patients face difficulties in making treatment decisions, particularly in relating clinical information to their personal circumstances. Two GPs felt patients are able to participate but still prefer to seek advice and confirmation from multiple clinicians that the correct decision is being made. Many reported that newly diagnosed patients often return to their GP to discuss the information and advice provided by their urologist. In addition, many patients, particularly elderly patients, assume that specialists know which treatment would be best and prefer to leave the choice to them as it gives the patient a feeling of safety.

Specialists reported giving patients information verbally during consultations, with occasional use of websites to support their explanation, along with a standard booklet about $\mathrm{PCa}$ and the pros and cons of different treatment options. GPs were mindful of giving informa- 
tion that does not conflict with the specialist's advice as they found it important to avoid complicating the treatment process and for the patient to have confidence in the specialist.

Both participant groups were open to using tools such as PDAs to inform patients and elicit their preferences. Four specialists felt that the PDA should be embedded in the treatment trajectory in a standardized manner to ensure that patients use it. Suggestions included enlisting oncology nurses to deliver the PDA to patients shortly after diagnosis and guide them in using it to understand their treatment options and reflect on their preferences. This was seen as advantageous as nurses are more accessible for patients and the trajectory can be more readily expanded to include additional contact time with them. One GP suggested that the PDA should also provide an easy way for the patient to contact his GP for further questions.

Two themes were unique to the GPs: trust and influence from multiple sources. This stemmed from patients' need for certainty about being cured and the confidence that their treatment achieves this. For this reason, GPs were unanimous that patients follow their urologist's treatment advice in the majority of cases. Other sources of influence according to two GPs are experiences and treatment decisions of fellow PCa sufferers, to the extent of preferring the same treatment with the expectation of having the same outcome. In other words, patients do not always view treatment options in terms of the impact on their own individual circumstances.

Specialists provided insight into additional logistical factors that may affect patient participation. The majority considered time constraints a major barrier. Consultation times varied by specialization; three urologists estimated that their consultations averaged 10-15 minutes; radiation oncologists reported having sufficient time (45-60 minutes) to discuss treatment details, and one nurse reported having 30-45 minutes per patient and more time if necessary. For this reason, one third of the specialists felt that oncology nurses should play a greater role in eliciting patient participation and supporting the SDM process as 
they were considered more approachable by patients and were able to have more contact time. The present role of oncology nurses is to provide patients with practical and logistical information about their treatment options, e.g. treatment procedures, side-effects, logistics of hospital visits, and so on. We did not find evidence that nurses engage with patients in deliberation or decision-making; this function appears to be performed solely by the urologist or radiation oncologist.

Five specialists highlighted the burden that SDM may place on the patient by making him responsible for treatment choices. Patients may feel overwhelmed by the volume of clinical information needed to make treatment decisions, may lack enough information to make an informed choice, or may experience stress and confusion by having to make a choice.

To counter this, two specialists suggested multidisciplinary clinics in which patients could discuss their options with specialists from different disciplines (urology, radiation oncology) under one roof. Although they recognized that this may bring logistical challenges, such as coordinating schedules among multiple clinicians, they felt that receiving information from both specializations at once may give patients a more balanced perspective of their options. 
Table 4.2: Core themes and sample quotes from GP and specialist interviews

\begin{tabular}{|c|c|}
\hline Theme & Sample quote \\
\hline \multirow[t]{5}{*}{$\begin{array}{l}\text { Patients' participation } \\
\text { preference/ability }\end{array}$} & $\begin{array}{l}\text { - "The very old people often think of a paternalistic model and believe that the doctor knows what's } \\
\text { good for them. I think most young people... would like to understand and have a say in what } \\
\text { treatment they are given." (Radiation oncologist 1) }\end{array}$ \\
\hline & $\begin{array}{l}-\quad \text { "[Patients] have to have a certain IQ to understand what we are saying and some don't." } \\
\text { (Radiation oncologist 2) }\end{array}$ \\
\hline & $\begin{array}{l}\text { - "For the patient it can be a barrier to have to make a choice for himself. We explain everything, } \\
\text { the pros and cons...but for the patient it can be very confusing." (Oncology nurse 2) }\end{array}$ \\
\hline & $\begin{array}{l}\text {-I think it also has to do with the generation, the older generation is much more used to the } \\
\text { hierarchical division of doctor-patient. They will follow what is presented. The younger generation } \\
\text { looks around the internet more and then comes to me with other options." (GP 1) }\end{array}$ \\
\hline & $\begin{array}{l}\text { - "Some patients are very good at making decisions, others have a bit the feeling of 'What should I } \\
\text { do?'" (GP 4) }\end{array}$ \\
\hline \multirow[t]{3}{*}{ Information provision } & $\begin{array}{l}\text { - "I think that you should first of all explain to patients that they have a choice and that they } \\
\text { themselves have an influence on that choice. Then you might be able to support them in using a } \\
\text { PDA by offering it structurally." (Urologist 2) }\end{array}$ \\
\hline & $\begin{array}{l}\text { - } \quad \text { "For some patients I use [clinical] guidelines to show that what the urologist has recommended } \\
\text { fits within international guidelines." (GP 5) }\end{array}$ \\
\hline & $\begin{array}{l}\text { - "In any case, the patients have to think about it for a longer period of time, especially about the } \\
\text { side effects. Patients should not quickly fill in a PDA in the waiting room. In order to encourage } \\
\text { this, it would be good if PDA said, for example, that if the patient has any questions, he can go to } \\
\text { the GP." (GP 8) }\end{array}$ \\
\hline Trust & - "Patients are very attached to the specialist's opinion. That is very important." (GP 2) \\
\hline $\begin{array}{l}\text { Influence of multiple } \\
\text { sources }\end{array}$ & $\begin{array}{l}\text { - } \quad \text { "There are [patients] who know someone with a very aggressive tumor and they are much more } \\
\text { likely to choose to have the tumor completely removed [with radical prostatectomy]." (GP 6) }\end{array}$ \\
\hline Time & $\begin{array}{l}\text { - "I think that [SDM] is much less efficient, it is very time-consuming.... It is far easier to just say } \\
\text { 'This is [the best option]'." (Radiation oncologist 2) }\end{array}$ \\
\hline SDM as a burden & $\begin{array}{l}\text { - }[\mathrm{SDM}] \text { asks a lot of the patient. ... We now place the choice with the patient and with it the } \\
\text { responsibility more or less is on him." (Urologist } 3 \text { ) }\end{array}$ \\
\hline \multirow[t]{2}{*}{ Treatment trajectory } & $\begin{array}{l}\text { - } \quad \text { "It would be great to have an oncology center, for example, where you can immediately discuss } \\
\text { everything with other specialists. But that will also be difficult." (Urologist 4) }\end{array}$ \\
\hline & $\begin{array}{l}\text { "The [hospital] must be ready [for] the extra logistical challenges to have two doctors in place of } \\
\text { one, and preferably at the same time or at the same clinic." (Radiation oncologist } 3 \text { ) }\end{array}$ \\
\hline
\end{tabular}

\subsection{Discussion}

In this study, we analyzed GPs' and specialists' perceptions regarding patient engagement in SDM in PCa management. GPs know patients and their background and motivations over a longer period of time compared to specialists. Their continuing role from diagnosis to posttreatment in providing psychosocial support puts them in a unique position to provide a holistic view of the patient experience $[11,13]$. PCa specialists, being the practitioners who lead the decision-making process, provided valuable input from the clinical perspective. Taken together, our results reveal that GPs and specialists have complemen- 
tary perspectives and competencies that can be harnessed to support and inform PCa patients.

Specialists cited patient age, challenges in processing clinical information, and predisposition to paternalistic models of decision-making as barriers to SDM. This perception is prevalent in many contexts [6] and may result in clinicians not prioritizing SDM due to preconceived notions that patients are unwilling or unable to engage [14]. As a result, PCa decisions are often heavily influenced by specialist recommendations [15]. We found confirmation of this in our interviews with GPs.

Interestingly, despite citing the high level of trust patients place in the specialist, our GPs reported that many patients still return to their GP after diagnosis for reassurance. Indeed, a Dutch survey of 4763 former cancer patients found that $82 \%$ desire more contact with their GP throughout their treatment, $68 \%$ consulted with their GP about their diagnosis, and that more frequent contact is associated with greater patient satisfaction [16]. The same study found that patients whose GP contacts them independently during treatment are more satisfied than when patients initiate the contact, indicating that a more well-defined role for the GP in the PCa trajectory may improve care quality.

A systematic review on patient-reported barriers to SDM corroborates our findings [17]. Patients prefer more contact time but perceive clinicians as being busy, resulting in some patients limiting consultation times themselves to avoid imposing on clinicians. Poor continuity of care, feelings of power imbalance, and a lack of trust further undermine their capacity to express their preferences. The authors conclude that structural changes are crucial to support patient participation.

These findings and our results reinforce the notion that while jointly evaluating high-quality clinical information is a key component of SDM, a trusting and empathic relationship between patients and their care providers is the foundation for effective patient engagement. Although GPs and nurses are able to provide the time necessary to build this relationship, and starting PCa treatment quickly is perhaps less urgent compared to many other cancer types, the deliberation 
process and treatment selection largely occur in consultations with specialists who face busy schedules and time constraints. Furthermore, prostate cancer trajectories are often fragmented, requiring patients to visit multiple specialists in different locations and at different times [18]. These factors may not only hinder patients in making choices aligned with their preferences but may also compromise their ability to participate in decision-making at all due to uncertainty and a perceived lack of control [19].

One structural factor proposed by our participants to alleviate these issues was the multidisciplinary clinic, in which patients consult with different specialists in one location (simultaneously or in sequence). This may provide patients with a sense of continuity and coordinated care that facilitates participation. PCa patients who participate in multidisciplinary clinics are more satisfied, less likely to be influenced by specialist bias [20], and their treatment patterns differ from patients seen by individual clinicians [21].

Second, it may be valuable to formalize the currently informal role of the GP by means of a post-diagnosis 'time out' consultation [22] in which patients and GPs review the information and treatment options provided by the specialist. GPs have the advantage of time and a longstanding relationship with the patient, which can be leveraged to not only help the patient understand clinical information but to evaluate it in light of his own preferences and situation. In this manner, the GP can help prepare the patient for the SDM conversation with the specialist. This would require GPs to develop new competencies in SDM and a redefinition of their role, as they currently prefer to align with specialists' recommendations in an attempt to preserve continuity. Coordination between GPs and specialists can facilitate this process and improve care quality [23].

Lastly, although integrating a PDA in the trajectory was considered important by our specialists, it is vital to consider the format in which the PDA is delivered. Often, PDAs are given to patients as supplementary material to take home, making it difficult to determine whether the pa- 
tient used the PDA correctly (if at all) and how it affected the decision process [9]. As a consequence, the patient may not be prepared enough to engage in the SDM talk. Since PDAs combined with a SDM talk are more effective than PDAs given as a stand-alone tool [24], practitioners could consider implementing both a regular PDA for home use as well as a simplified version that clinicians can use in consultations to stimulate dialogue and patient engagement. Decision cards are an example of a simplified PDA that can help structure the consultation and ensure that the essential components of SDM are addressed [25].

Figure 4.2 depicts how these three factors may be incorporated into a PCa care pathway under a traditional three-talk SDM model [3]. The three-talk model conceptualizes SDM as consisting of three components: a choice talk, in which the patient is made aware that there is choice to be made between various options; an option talk, in which the clinician provides the patient with detailed information on the treatment options; and a decision talk, in which the options are evaluated in terms of the patient's preference and a choice is made jointly by the patient and their clinician. These talks need not necessarily take place at separate time points. For instance, in our context, the traditional care pathway (Panel A) begins with referral from to a specialist from the GP. The patient then undergoes diagnostic testing (1st consultation), receives the diagnosis and basic treatment information (2nd consultation), and then the multidisciplinary tumor board's treatment advice through a series of consultations with a urologist, supported by oncology nurses. The treatment decision is usually made in the 3rd consultation with the specialist based on the tumor board's advice, the clinician's advice, and if possible the patient's own preference. In this instance, the option talk and decision talk may be combined in the 3rd consultation. However, time constraints can make combined talks suboptimal as the patient may not be in a position to evaluate and weigh his options as well as make a treatment decision in one consultation.

The alternative care pathway shown in Panel B of Figure 4.2 incorporates the three structural factors mentioned previously: (1) a time out 
consultation with the GP soon after the patient receives the diagnosis; (2) decision support (such as a PDA and/or decision cards) embedded in the pathway; (3) a multidisciplinary consultation in place of the 3rd consultation with the urologist. GPs and patients can use the time out consultation to go over the information and advice presented by the specialist and relate it to the patient's situation. The GP can elicit the patient's values and preferences and help him prepare for the final SDM talk in the multidisciplinary clinic. In this manner, treatment decisions can be made based on both the best available clinical evidence as well as clearly articulated patient preferences.

These care pathways can be evaluated with validated instruments along multiple dimensions, such as the OPTION scale [26], the SDM-Q-9 and/or SDM-Q-Doc that measure patient and clinician participation respectively [27], and the decisional conflict scale [28]. However, these metrics should be supplemented with in-depth interviews and focus groups with patients to understand how they experience these initiatives, particularly regarding trust, which remains a gap in the literature.

While much of the SDM literature focuses on the clinical interaction between patient and specialist, our study provides a deeper insight into the distributed nature of PCa decision-making across primary and secondary care [29]. Nevertheless, it is important to note its limitations. First, since our sample is small and limited to one clinic, care should be taken when extrapolating our results to different contexts. Although our results corroborate the literature, implementation efforts must be adapted to the local context. Second, our findings may be strengthened by considering the role of oncology nurses in greater detail as they were underrepresented in our study. Our interviews with nurses revealed that their present function centers more on information provision than deliberation and decision-making. Findings from other contexts also show that nurses are not trained specifically in SDM and highlight the importance of nurses receiving such training [30], since SDM is not only relevant to major treatment decisions but also smaller decisions along the trajectory where nurse support is invaluable. 
A

Traditional care pathway:

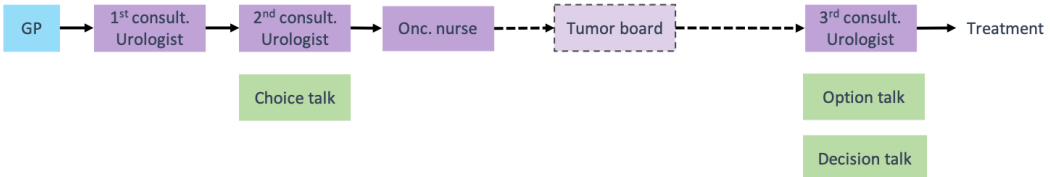

B

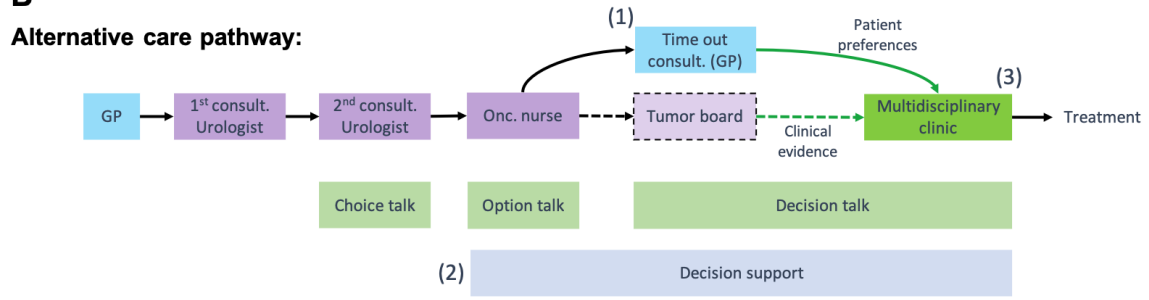

Figure 4.2: A comparison of the traditional care pathway (Panel A) and an alternative care pathway (Panel B) based on our findings. The traditional care pathway followed by a PCa patient, as described by specialists in our study, involves a series of consultations with the urologist after referral from a GP. The multidisciplinary tumor board is represented with dashed lines to indicate that the patient is not present in this part of the pathway. The green boxes indicate components of the traditional three-talk SDM model [3]. Implementing the three-talk model in such a pathway can result in time-constrained consultations, in which detailed information about treatment options relevant to the patient (option talk) and the patient's preferences (decision talk) are discussed in one consultation with the urologist. Under the alternative care pathway with a post-diagnosis time out consultation (1), modified decision support tools (2), and a multidisciplinary consultation (3), the decision talk may be spread over time and across specializations so that the patient can make an informed choice based on his preferences as well as the best available clinical evidence. 


\subsection{Conclusion}

Despite clinicians' positive attitudes towards SDM, the current level and type of information provision seems insufficient to engage PCa patients successfully. GPs and PCa specialists possess complementary skills that can be integrated into the care pathway so that patients can benefit from both clinical expertise and a trusting environment. If SDM in PCa is to be supported, clinicians across the care pathway must be sensitive to the factors that hinder patient participation. Our study suggests structural facilitators that may empower patients to share their values and preferences. In order for these facilitators to be implemented successfully, both patient and clinician perspectives must be taken into account in their design, and the effects must be measured using an appropriate methodology. 


\section{References}

1. Keyes M, Crook J, Morton G, Vigneault E, Usmani N, Morris WJ. Treatment options for localized prostate cancer. Can. Fam. Physician Med. Fam. Can. 2013;59:1269-74.

2. Wollersheim BM, van Stam M-A, Bosch RJLH, Pos FJ, Tillier CN, van der Poel HG, et al. Unmet expectations in prostate cancer patients and their association with decision regret. J. Cancer Surviv. 2020;14:731-8.

3. Elwyn G, Frosch D, Thomson R, Joseph-Williams N, Lloyd A, Kinnersley $P$, et al. Shared decision making: a model for clinical practice. J. Gen. Intern. Med. 2012;27:1361-7.

4. Patiëntenfederatie Nederland. Samen Beslissen [Internet]. Utrecht: Netherlands Patients Federation; 2020. Available from: https://www.patientenfederatie.nl/downloads/rapporten/200-rapportsamen-beslissen/file

5. Vos M de, Centrum voor Ethiek en Gezondheid. Wanneer samen beslissen niet vanzelf spreekt: reflecties van patiënten en artsen over gezamenlijke besluitvorming. 2014.

6. Légaré F, Ratté S, Gravel K, Graham ID. Barriers and facilitators to implementing shared decision-making in clinical practice: update of a systematic review of health professionals' perceptions. Patient Educ. Couns. 2008;73:526-35.

7. Lambin P, Zindler J, Vanneste BGL, De Voorde LV, Eekers D, Compter I, et al. Decision support systems for personalized and participative radiation oncology. Adv. Drug Deliv. Rev. 2017;109:131-53.

8. Stacey D, Légaré F, Lewis KB. Patient decision aids to engage adults in treatment or screening decisions. Jama 2017;318:657-8.

9. Agoritsas T, Heen AF, Brandt L, Alonso-Coello P, Kristiansen A, Akl EA, et al. Decision aids that really promote shared decision making: the pace quickens. BMJ 2015;g7624.

10. Elwyn G, Frosch DL, Kobrin S. Implementing shared decision-making: consider all the consequences. Implement. Sci. 2015;11:114.

11. Merriel SW, Gnanapragasam V. Prostate cancer treatment choices: the GP's role in shared decision making. Br. J. Gen. Pract. 2019;69:588-9. 
12. Ankolekar A, Vanneste BGL, Bloemen-van Gurp E, van Roermund JG, van Limbergen EJ, van de Beek K, et al. Development and validation of a patient decision aid for prostate Cancer therapy: from paternalistic towards participative shared decision making. BMC Med. Inform. Decis. Mak. 2019;19:130.

13. Dunn J, Ralph N, Green A, Frydenberg M, Chambers SK. Contemporary consumer perspectives on prostate cancer survivorship: Fifty voices. Psychooncology. 2020;29:557-63.

14. Politi MC, Dizon DS, Frosch DL, Kuzemchak MD, Stiggelbout AM. Importance of clarifying patients' desired role in shared decision making to match their level of engagement with their preferences. BMJ 2013;347:f7066-f7066.

15. Kim SP, Gross CP, Nguyen PL, Nguyen PY, Smaldone MC, Thompson RH, et al. Specialty bias in treatment recommendations and quality of life among radiation oncologists and urologists for localized prostate cancer. Prostate Cancer Prostatic Dis. 2014;17:163-9.

16. Noteboom EA, Perfors IA, May AM, Stegmann ME, Duijts SF, Visserman EA, et al. GP involvement after a cancer diagnosis; patients' call to improve decision support. BJGP Open 2021;5.

17. Joseph-Williams N, Elwyn G, Edwards A. Knowledge is not power for patients: A systematic review and thematic synthesis of patient-reported barriers and facilitators to shared decision making. Patient Educ. Couns. 2014;94:291-309.

18. Gomella LG. The Prostate Cancer Unit: A Multidisciplinary Approach for Which the Time Has Arrived. Eur. Urol. 2011;60:1197-9.

19. Berry LL, Davis SW, Wilmet J. When the customer is stressed. Harv. Bus. Rev. 2015;93:86-94.

20. Hurwitz LM, Cullen J, Elsamanoudi S, Kim DJ, Hudak J, Colston M, et al. A prospective cohort study of treatment decision-making for prostate cancer following participation in a multidisciplinary clinic. Urol. Oncol. Semin. Orig. Investig. 2016;34:233.e17-233.e25.

21. Korman H, Lanni T, Shah C, Parslow J, Tull J, Ghilezan M, et al. Impact of a Prostate Multidisciplinary Clinic Program on Patient Treatment Decisions 
and on Adherence to NCCN Guidelines: The William Beaumont Hospital Experience. Am. J. Clin. Oncol. 2013;36:121-5.

22. de Wit NJ. A "time out consultation" in primary care for elderly patients with cancer: Better treatment decisions by structural involvement of the general practitioner. Eur. J. Cancer Care (Engl.) 2017;26:e12711.

23. Druel V, Gimenez L, Paricaud K, Delord J-P, Grosclaude P, Boussier N, et al. Improving communication between the general practitioner and the oncologist: a key role in coordinating care for patients suffering from cancer. BMC Cancer 2020;20:495.

24. Baptista S, Teles Sampaio E, Heleno B, Azevedo LF, Martins C. Web-Based Versus Usual Care and Other Formats of Decision Aids to Support Prostate Cancer Screening Decisions: Systematic Review and Meta-Analysis. J. Med. Internet Res. 2018;20:e228.

25. de Mik SML, Stubenrouch FE, Balm R, Ubbink DT. Development of three different decision support tools to support shared decision-making in vascular surgery. Patient Educ. Couns. 2021;104:282-9.

26. Elwyn G, Hutchings H, Edwards A, Rapport F, Wensing M, Cheung W, et al. The OPTION scale: measuring the extent that clinicians involve patients in decision-making tasks. Health Expect. 2005;8:34-42.

27. Kriston L, Scholl I, Hölzel L, Simon D, Loh A, Härter M. The 9-item Shared Decision Making Questionnaire (SDM-Q-9). Development and psychometric properties in a primary care sample. Patient Educ. Couns. 2010;80:94-9.

28. O'Connor AM. Validation of a decisional conflict scale. Med. Decis. Mak. Int. J. Soc. Med. Decis. Mak. 1995;15:25-30.

29. Rapley T. Distributed decision making: the anatomy of decisions-inaction: Doctor-patient relationships and distributed decision making. Sociol. Health Illn. 2008;30:429-44.

30. Lenzen SA, Daniëls R, van Bokhoven MA, van der Weijden T, Beurskens A. What makes it so difficult for nurses to coach patients in shared decision making? A process evaluation. Int. J. Nurs. Stud. 2018;80:1-11. 



\section{Clinician perspectives on clinical decision support systems in lung cancer: Implications for shared decision-making}

Ankolekar, A., Van Der Heijden, B., Dekker, A., Roumen, C., De Ruysscher, D., Reymen, B., Berlanga, A., Oberije, C., \& Fijten, R.

Submitted 


\section{Abstract}

\section{Background}

Lung cancer treatment decisions are typically made among clinical experts in a multidisciplinary tumor board (MTB) based on clinical data and guidelines. The rise of artificial intelligence and cultural shifts towards patient autonomy are changing the nature of clinical decisionmaking towards personalized treatments. This can be supported by clinical decision support systems (CDSSs) that generate personalized treatment information as a basis for shared decision-making (SDM). Little is known about lung cancer patients' treatment decisions and the potential for SDM supported by CDSSs.

\section{Objective}

To explore (1) the extent to which patient preferences are taken into consideration in non-small cell lung cancer (NSCLC) treatment decisions; (2) clinician perspectives on using CDSSs to support SDM.

\section{Design}

Mixed methods study consisting of a retrospective cohort study on patient deviation from MTB advice and reasons for deviation, qualitative interviews with lung cancer specialists, and observations of MTB discussions and patient consultations.

\section{Setting and participants}

NSCLC patients $(\mathrm{N}=257)$ treated at a single radiotherapy clinic and nine lung cancer specialists from six Dutch clinics.

\section{Results}

We found a $10.9 \%(n=28)$ deviation rate from MTB advice; $50 \%(n=14)$ were due to patient preference, of which $85.7 \%(n=12)$ chose a less intensive treatment than MTB advice. Current MTB recommendations are based on clinician experience, clinical guidelines, and patients' performance status. Most specialists $(n=7)$ were receptive towards CDSSs but cited barriers such as lack of trust, lack of validation studies, and 
time. CDSSs were considered valuable during MTB discussions rather than in consultations.

\section{Conclusion}

Lung cancer decisions are heavily influenced by clinical guidelines and experience, yet many patients prefer less intensive treatments. CDSSs can support SDM by presenting the harms and benefits of different treatment options rather than giving a single treatment advice. External validation of CDSSs should be prioritized.

\section{Patient or public contribution}

This study's objectives and data collection were supported by Dutch health care insurer CZ for a project titled 'My Best Treatment' that improves patient-centeredness and the lung cancer patient pathway in the Netherlands. 



\subsection{Introduction}

Lung cancer is a leading source of cancer mortality worldwide with a poor prognosis and 5-year survival rate of $17.8 \%$ [1,2]. Depending on the tumor stage, treatments include surgery, chemotherapy, radiotherapy, chemoradiation, and immunotherapy [3]. Treatment decisions are typically made in multidisciplinary tumor boards (MTBs) where pulmonologists, radiation oncologists, surgeons, medical oncologists, nurses and other specialists discuss the patient's options in light of the latest evidence and clinical guidelines [4]. Decision-making has traditionally been based on clinician knowledge, experience, and international/regional guidelines, and lung cancer patients largely follow clinician advice [5]. In recent years however, shared decision making (SDM) has gained prominence. SDM is the collaborative process between patient and clinician to make treatment decisions that strike a balance between clinical evidence and patient preferences [6]. Under this approach, a patient and their clinician may decide together to deviate from the MTB advice and choose treatments that are more in line with their preferences [7-9]. A large-scale survey of Dutch lung cancer patients found that $85 \%$ would like to be involved in the treatment decision [10]. The same study found that patients experience the most decisional conflict with uncertainty, feeling informed, and clarifying their values. A previous study by the same authors found that the majority of Dutch lung cancer specialists also felt that patients should be involved in their treatment decisions but that the two biggest barriers are time constraints and the difficulty some patients experience in weighing their treatment options [11]. This suggests that better decision support may play a valuable role in helping patients and clinicians to evaluate the harms and benefits of different treatment options.

The growth of data-driven health care is introducing a predictive element to the treatment choices for which SDM is crucial. Artificial intelligence (AI) may help clinicians (and patients) quantify the risks and benefits of various treatment options based on individual patient characteristics to determine which treatment may benefit an individual patient the most [12]. Building these predictive tools 
into a clinical decision support system (CDSS) enables clinicians to move from population-based evidence to more individualized approaches. A systematic review of ten implemented CDSSs for treatment choices in oncology shows that five had a statistically significant positive effect on process outcomes such as treatment adherence, and four were associated with an improvement in patient outcomes [13]. There is further evidence that individualized radiotherapy schedules provide survival benefits over conventional radiotherapy and chemotherapy treatments for lung cancer patients [14]. However, there is a lack of adequate decision support for lung cancer. A systematic review of lung cancer CDSSs found 39 CDSSs in total estimating overall survival or progression-free survival based on demographics and lifestyle factors (age, gender, use of tobacco), physical factors (performance status, body-mass index (BMI)), tumor characteristics (tumor staging, metastases), treatment characteristics (time from diagnosis to treatment, prior treatment response), serum markers, and genetic markers [15]. The authors concluded that the value of the majority of these CDSSs was limited due to the use of old clinical data, lack of large-scale validation, and lack of user-friendliness.

As with any new development, AI-driven CDSSs face various implementation challenges and the majority of CDSSs do not reach usual care [16]. Aside from addressing technical challenges such as usability, there is a need to focus on cultural issues such as clinician attitudes towards CDSSs as well, since these technologies may transform traditional decision-making processes, norms, and hierarchies $[17,18]$.

The objective of this study was to determine the extent to which lung cancer patients' preferences are currently taken into consideration in decision-making, and whether clinical decision support may facilitate $\mathrm{SDM}$. We use a mixed methods design to investigate lung cancer treatment decisions and deviations from MTB advice to gauge the current level of patient participation at our clinic. We also conduct qualitative interviews with Dutch lung cancer specialists to gain insight into the current decision-making process and to determine their perspectives 
on the barriers and facilitators to SDM in the lung cancer trajectory, using CDSSs to support SDM, possible barriers to CDSSs, and how these barriers might be alleviated.

\subsection{Methods}

We followed a mixed methods design consisting of a sequential quantitative and qualitative phase. The quantitative phase consisted of a retrospective cohort study to answer the following research questions: (1) What percentage of non-small cell lung cancer (NSCLC) patients deviate from MTB treatment recommendations and what are the reasons for deviation? (2) What is the impact of deviation on treatment outcomes? Following our finding that the level of patient deviation was low and patients tend to follow MTB recommendations, we conducted the qualitative study to investigate possible reasons. We interviewed lung cancer specialists to gain insight into the current decisionmaking process, clinicians' attitudes towards SDM and CDSS, and possible implementation barriers. The qualitative phase was guided by the following questions: (1) Which factors influence MTB decisions? (2) To what extent do clinicians engage patients in SDM in consultations? (3) What role may CDSSs play in supporting decision-making and at which point in the treatment trajectory should a CDSS be implemented?

The advantage of the retrospective cohort study design is that it uses historical data routinely collected in electronic health records (EHRs) and is relatively accessible, thereby providing an efficient way to address the first two research questions. One drawback is selection bias, as we include patients referred to our clinic for radiotherapy treatment. Patients who deviated from MTB advice that did not involve radiotherapy were not included and therefore the actual percentage of patient deviations may differ. The qualitative study compensates to an extent by including the perspectives of pulmonary oncologists who oversee the full lung cancer treatment trajectory and provide insights into the process of MTBs, decision-making, and patient consultations. 
Chapter 5. Clinician perspectives on clinical decision support systems in lung cancer

This combined approach provides a more comprehensive view of the lung cancer treatment trajectory.

\subsubsection{Ethics Approval}

Our institute's Internal Review Board (IRB) reviewed and approved both parts of this study.

\subsubsection{Retrospective cohort study}

\section{Patient population}

We included all stage I-IIIB inoperable non-metastasized NSCLC patients discussed at MTBs during 2014-2015 and treated at our institute who met the following inclusion criteria: (1) the first choice treatment was curatively intended primary (chemo)radiotherapy due to unresectable disease and/or medical inoperability; (2) WHO performance status of 0-2 (where ' 0 ' refers to the ability to carry out all normal activities without restriction, ' 1 ' refers to restriction in physically strenuous activity but otherwise capable of walking and carrying out light work, and ' 2 ' refers to the ability for self-care but not work activities); (3) no history of prior chest radiotherapy or lung surgery, no other active malignancy. This yielded a sample of 257 patients.

\section{Data collection}

The following data was collected in February 2017 by $\mathrm{BH}$ from patients' EHRs: general characteristics (age at diagnosis, gender, WHO performance status), tumor characteristics (histology and staging); MTB treatment advice, final treatment decision and reason for any deviation; treatment outcomes (survival rate, recurrence, toxicity, quality of life, treatment compliance, and adverse events). These data are typically recorded in the EHR by pulmonary oncologists, assistants, and nurses. Survival rates were reported for a maximum of three years as the follow up time was between two and three years for patients treated in 2014 and between one and two 
years for patients treated in 2015, depending on the date of diagnosis. In addition, quality of life was measured by the EQ-5D questionnaire [19], the Visual Analogue Scale [20], and the EORTC QLQ-C30 [21]. These questionnaires were administered just before the treatment and two and six weeks after the last radiation treatment.

\section{Data Analysis}

We calculated the percentage of patients who deviated from the treatment advised by the MTB, and the reasons for the deviations. Additional analysis on the effects on outcomes and quality of life were performed and are presented in the appendix.

\subsubsection{Qualitative study}

We used a semi-structured interview format covering the following topics: (i) current lung cancer treatment trajectory and MTB process; (ii) patient communication in consultations and possibilities for SDM; (iii) participants' attitudes towards prognostic prediction models and implementation requirements. In addition, we obtained permission from the specialists we interviewed to conduct field observations of MTB discussions and patient consultations at the clinics we studied. The purpose of these observations was to gain a deeper understanding of the process of MTB discussions and the nature of the patientclinician interaction.

\section{Participants}

Participants were recruited using purposive sampling [22]. Initial interviews were carried out with pulmonary oncologists within our institute who then referred us to clinicians in four medical centers across the Netherlands. Six clinics were represented in our study; four in the southern province of Limburg, and two in northern provinces. Our aim was to ensure a variety of perspectives and backgrounds, therefore we included clinicians from different age groups, experience levels, and specializations. Specific knowledge about AI was not required. 


\section{Data collection}

Data was collected during February-March 2017. AA, BH, CR, and AB conducted the qualitative interviews and $\mathrm{AA}$ and $\mathrm{BH}$ performed field observations. Participants were interviewed in-person at their workplace and interviews were audio-recorded after obtaining written consent from the participant. The mean interview duration was 48 minutes, with a standard deviation of 14.1 minutes. All interviews were transcribed and subsequently reviewed and approved by the participants.

\section{Data Analysis}

Interviews were analyzed using thematic analysis [23]. This method was chosen for its flexibility and the exploratory nature of this study. Each transcript was read multiple times along with hearing the audio recording, and then fragments of text were assigned labels summarizing their content. These codes were then reviewed and grouped under the themes that emerged. Finally, the themes were reviewed in light of the text extracts to check whether they reflected the data. Interviews were coded independently and the codes were cross-checked by two researchers to improve validity. Data was gathered until saturation, i.e. no new themes emerged.

\subsection{Results}

5.3.1 Retrospective cohort study results: patient deviation from MTB advice

We reviewed the treatment decisions and potential changes of 257 NSCLC patients treated with radiotherapy; 229 patients $(89.1 \%)$ followed MTB advice and $28(10.9 \%)$ deviated. Both patient groups were comparable in terms of age, gender, WHO performance status, and tumor grade, as shown in Table 5.1. For this a one sample z-test was used. The two groups did not differ significantly on survival Mean overall survival was 23.9 months (95\% CI 22.2-25.6 months). In 
the group of patients who followed MTB advice the mean survival was 23.2 months (95\% CI 21.5-25.0 months). Patients who did not follow the MTB advice had a mean survival of 24.6 months $(95 \%$ CI 19.4-29.8 months). This difference in survival between the two groups was not significant according to the log-rank test (p-value: 0.707$)$. The two groups also did not differ significantly in terms of outcomes, and only slightly on quality of life (comparisons of survival, recurrence, toxicity, and quality of life are presented in the Appendix).

Fifty percent of the deviations were due to patients' preference (Fig. 5.1). Of these, two patients preferred a more intensive treatment (concurrent chemoradiation in place of sequential, and curative radiotherapy in place of palliative), while twelve patients preferred a less intensive treatment, namely: radiotherapy in place of chemoradiation (8 patients), sequential in place of concurrent chemoradiation (3 patients), or chemoradiation at a lower dose (1 patient). Thirteen patients $(46.4 \%)$ deviated due to medical infeasibility, e.g. tumor progression or loss of fitness. 
Chapter 5. Clinician perspectives on clinical decision support systems in lung cancer

Table 5.1: Demographic and clinical characteristics of NSCLC patients in cohort study.

\begin{tabular}{|c|c|c|c|c|}
\hline Characteristic & $\begin{array}{l}\text { All patients } \\
(n=257)\end{array}$ & $\begin{array}{l}\text { Patients who } \\
\text { followed MTB } \\
\text { advice } \\
(\mathrm{n}=229)\end{array}$ & $\begin{array}{l}\text { Patients who } \\
\text { deviated from } \\
\text { MTB advice } \\
(\mathrm{n}=28)\end{array}$ & p-value \\
\hline Mean age (years) & 68.9 & 68.5 & 71.9 & 0.078 \\
\hline \multicolumn{5}{|l|}{ Gender } \\
\hline Male & $158(61.5 \%)$ & $136(59.4 \%)$ & $22(78.6 \%)$ & \multirow[t]{2}{*}{0.063} \\
\hline Female & $99(38.5 \%)$ & $93(40.6 \%)$ & $6(21.4 \%)$ & \\
\hline \multicolumn{5}{|c|}{ WHO performance status } \\
\hline 0 & $35(13.6 \%)$ & $33(14.4 \%)$ & $2(7.1 \%)$ & \multirow[t]{3}{*}{0.389} \\
\hline 1 & $177(68.9 \%)$ & $158(69 \%)$ & $19(67.9 \%)$ & \\
\hline 2 & $45(16.6 \%)$ & $38(16.6 \%)$ & $7(25 \%)$ & \\
\hline \multicolumn{5}{|l|}{ Histology } \\
\hline Adenocarcinoma & $87(33.9 \%)$ & $78(34.1 \%)$ & $9(32.1 \%)$ & \multirow[t]{3}{*}{0.653} \\
\hline $\begin{array}{l}\text { Squamous cell } \\
\text { carcinoma }\end{array}$ & $120(46.7 \%)$ & $105(45.9 \%)$ & $15(53.6 \%)$ & \\
\hline Other & $50(19.5 \%)$ & $46(20.1 \%)$ & $4(14.3 \%)$ & \\
\hline \multicolumn{5}{|l|}{ NSCLC stage } \\
\hline IA & $29(11.3 \%)$ & $27(11.8 \%)$ & $2(7.1 \%)$ & \multirow[t]{6}{*}{0.940} \\
\hline IB & $1(0.4 \%)$ & $12(5.2 \%)$ & $1(3.6 \%)$ & \\
\hline IIA & $5(1.9 \%)$ & $4(1.7 \%)$ & $1(3.6 \%)$ & \\
\hline IIB & $13(5.1 \%)$ & $12(5.2 \%)$ & $1(3.6 \%)$ & \\
\hline IIIA & $91(35.4 \%)$ & $81(35.4 \%)$ & $10(35.7 \%)$ & \\
\hline IIIB & $106(41.2 \%)$ & $93(40.6 \%)$ & $13(46.6 \%)$ & \\
\hline
\end{tabular}



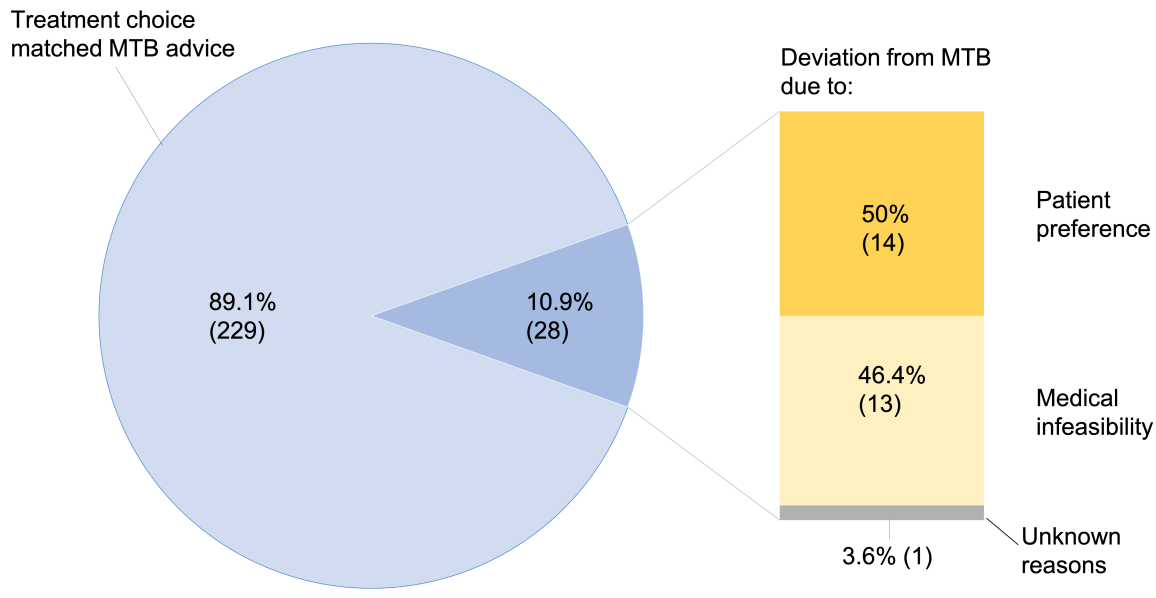

Figure 5.1: Treatment decisions in NSCLC patients following the MTB and reasons for deviation from MTB advice

\subsubsection{Qualitative study results}

Nine clinicians were interviewed for the qualitative part of the study: six pulmonologists, two radiation oncologists, and one oncology nurse. The clinicians' experience ranged from 6 to 25+ years with an average of 10-14 years of experience (Table 5.2). All clinicians reported having basic familiarity with SDM and CDSSs: four had knowledge of CDSSs in general, two were familiar with CDSSs for disease areas outside lung cancer (namely, breast cancer), and three had knowledge of specific lung cancer CDSSs, with one out of these three using Brock and Herder prediction models occasionally to predict the risk that a pulmonary nodule is lung cancer [24,25].

\section{Current decision-making process}

Clinicians described the typical treatment trajectory of a lung cancer patient as follows: after performing diagnostic testing to confirm the presence of lung tumors, the pulmonary oncologist presents the pa- 
Chapter 5. Clinician perspectives on clinical decision support systems in lung cancer

Table 5.2: Characteristics of clinicians who participated in the study

\begin{tabular}{ll}
\hline Characteristic & $\mathbf{N}$ \\
\hline Age (yrs) & $2(22.2 \%)$ \\
$30-39$ & $1(11.1 \%)$ \\
$40-49$ & $4(44.4 \%)$ \\
$50-59$ & $2(22.2 \%)$ \\
$60-69$ & \\
Gender & $3(33.3 \%)$ \\
Male & $6(66.7 \%)$ \\
Female & \\
Specialization & $6(66.7 \%)$ \\
Pulmonary oncologist & $2(22.2 \%)$ \\
Radiation oncologist & $1(11.1 \%)$ \\
Oncology nurse & \\
Experience (yrs) & $1(11.1 \%)$ \\
$5-9$ & $4(44.4 \%)$ \\
$10-14$ & $1(11.1 \%)$ \\
$15-19$ & $1(11.1 \%)$ \\
$20-24$ & $2(22.2 \%)$ \\
$25+$ &
\end{tabular}

tient's case in the weekly MTB which is typically attended by pulmonary oncologists, pathologists, radiation oncologists, surgeons, radiologists, and oncology nurses. The MTB generates possible treatment options (surgery, chemotherapy, radiotherapy, immunotherapy, targeted agents, or a combination) based on clinical guidelines and patient data (e.g. age, health status, lung function, comorbidities, blood and other lab values, tumor stage, location, and genomic markers). The three most commonly cited factors influencing treatment decisions were the clinicians' own experience (eight out of nine clinicians), clinical guidelines, and the patient's WHO performance status (six out of nine clinicians in both cases). Additional factors were clinical studies, scientific literature, and patient preferences. There was no evidence of 
CDSSs being used to support MTB discussions in the clinics studied. The pulmonary oncologist then presents the MTB advice to the patient and the treatment is then decided. The pulmonary oncologist is the lead clinician throughout the treatment trajectory and therefore would typically carry out a SDM consultation. When radiotherapy is advised, the patient is referred to a radiation oncologist, who may also engage in SDM for decisions within the radiation oncology domain.

\section{Clinician attitudes towards SDM in lung cancer}

Table 5.3 summarizes the main factors that may hinder or support SDM according to our clinicians. Most had mixed views regarding the scope for SDM in lung cancer, emphasizing that there are rarely multiple treatment options for lung cancer patients since the clinical situation and guidelines usually point towards a single treatment that offers the best chance of success. Certain treatments, such as radiotherapy, offer more scope for an individualized approach, according to one clinician, however two clinicians pointed out that lung cancer patients rarely ask about alternative treatment schedules. If the MTB generates multiple treatment possibilities, all interviewed clinicians said that they engage in deeper discussion with the patient about the best course of action. In these situations, clinicians reported practicing SDM to varying degrees; for two clinicians it was important to present the options to the patient and then let the patient decide without the clinician presenting their own opinion, while one clinician reported sharing her own opinion about the most appropriate treatment option with the patient. When asked about factors that would facilitate the SDM conversation, two clinicians mentioned the value of tools that help patients to clarify their preferences. These were seen as beneficial for both the patient as well as the clinician, as currently patient preferences are seldom made explicit. Three clinicians mentioned that additional time in the consultation would be valuable to engage with the patient and answer their questions.

\section{Clinician attitudes towards CDSS}

Four themes regarding CDSS implementation emerged from our analysis (summarized in Table 5.4): (I) opinions on the added value of 
Table 5.3: Clinician perspectives on factors that influence the possibility of SDM in the lung cancer trajectory

\begin{tabular}{|c|c|c|}
\hline Theme & Factor & Sample quote \\
\hline \multirow[t]{2}{*}{$\begin{array}{l}\text { Applicability of SDM in } \\
\text { lung cancer }\end{array}$} & Rarely multiple options & $\begin{array}{l}\text { "If there are several options, then [SDM is } \\
\text { appropriate]. But very often you already have one } \\
\text { preferred option that has the best chance of success. } \\
\text { See, only at stage I, radiation versus surgery - that's a } \\
\text { clear one. But otherwise you very often have one } \\
\text { treatment that is preferred." (Clinician 1) }\end{array}$ \\
\hline & $\begin{array}{l}\text { More suitable within certain } \\
\text { radiotherapy treatments }\end{array}$ & $\begin{array}{l}\text { "[SDM] is mainly for the group when it comes to } \\
\text { radiation only - which fractionation schemes, yes, that } \\
\text { is something you decide together with the patient." } \\
\text { (Clinician } 7 \text { ) }\end{array}$ \\
\hline \multirow[t]{3}{*}{ Facilitators to SDM } & Tool to clarify patient preferences & $\begin{array}{l}\text { "What might help is some kind of app or a form where } \\
\text { the side-effects are plotted and where the patient can } \\
\text { give a score, which [they] then go and discuss } \\
\text { together with the doctor: 'These are the side-effects } \\
\text { that could occur, here you may have a lot of difficulty, } \\
\text { is it so much trouble that you would not want the } \\
\text { treatment?" (Clinician } 2 \text { ) }\end{array}$ \\
\hline & & $\begin{array}{l}\text { "I think [decision aids] can be very enlightening for the } \\
\text { patient, but also for the doctor. Like: 'What are you } \\
\text { actually choosing between?' It remains somewhat } \\
\text { vague now." (Clinician 8) }\end{array}$ \\
\hline & Additional consultation time & $\begin{array}{l}\text { "With me [patients] always get the time they need but I } \\
\text { still think 'Gosh, they really need more time,' because } \\
\text { they always ask the same question every time. So } \\
\text { perhaps I have not been really clear. [...] There's also } \\
\text { emotion at play." (Clinician } 4 \text { ) }\end{array}$ \\
\hline
\end{tabular}

CDSSs, (II) trust, (III) risk communication and (IV) time constraints. The majority (seven out of nine clinicians) had positive views regarding the potential of CDSSs in general, yet three felt that clinical guidelines determine the best treatment based on survival chances and that treatment decisions do not change often enough for a CDSS to add value. In addition, they noted that treatment choices are affected by factors such as the patient's fitness level, so that even if a CDSS provides a prognostic prediction favoring a certain treatment, the clinician would still recommend a treatment based on what the patient can handle. In addition, two clinicians mentioned that in contrast to other more preference-sensitive cancer types, lung cancer treatment side-effects do not play a deciding role in treatment decisions, as the side-effects are transitory in nature. As a result, six clinicians felt that 
CDSSs would be more useful before or during the MTB, rather than in the consultation with patients. Specifically, three considered it useful when a CDSS presents the relevant variables, such as patient characteristics and clinical data, and generates survival predictions for different treatment options. Two clinicians felt CDSSs could be used after the MTB as supporting material in the consultation to present the treatment advice to the patient.

The second most prevalent challenge was interpreting risk predictions made by a CDSS and communicating these to patients who often find it difficult to interpret these estimates. There was a perception that CDSSs that predict survival outcomes are not useful for patients, as extreme predictions may place unnecessary stress and middle-of-theroad predictions are not useful in decision-making. In addition, survival prognosis in lung cancer is lower than in other disease areas and three clinicians mentioned that it can be challenging to present the patient with low numbers.

The lack of large-scale multi-center validation studies made three clinicians wary of using CDSSs in practice. They questioned whether CDSSs based on certain data could be useful in generating predictions for other populations or patients with a different profile. In addition, they highlighted the challenge in measuring CDSS performance over time, such as checking outcome predictions against actual outcomes. Time constraints were mentioned by two clinicians as a factor that might dissuade their peers from using a CDSS. This included the time taken to use the CDSS itself, such as filling in patient variables into the system, as well as to explain and discuss output in consultations with the patient. 
Table 5.4: Barriers to CDSS implementation in lung cancer pathway according to clinicians

\begin{tabular}{|c|c|c|}
\hline $\begin{array}{l}\text { Barriers to } \\
\text { CDSS }\end{array}$ & Factor & Sample quote \\
\hline \multirow[t]{2}{*}{$\begin{array}{l}\text { Value for } \\
\text { clinicians }\end{array}$} & Clinicians' own experience & $\begin{array}{l}\text { "We know what the survival curves for lung cancer look } \\
\text { like for the different stages. We have them all in our } \\
\text { heads along with the respective treatment options. We } \\
\text { use that to determine the correct treatment strategy for } \\
\text { someone, what their chances are." (Clinician } 7 \text { ) }\end{array}$ \\
\hline & Prevalence of one 'best' treatment & $\begin{array}{l}\text { "Often there aren't that many choices, so I find that very } \\
\text { difficult. Yes, you can discuss treating versus not } \\
\text { treating, but often there are not very many treatment } \\
\text { options." (Clinician 2) }\end{array}$ \\
\hline \multirow[t]{2}{*}{$\begin{array}{l}\text { Value for } \\
\text { patients }\end{array}$} & Difficult for patients to interpret predictions & $\begin{array}{l}\text { "If there is a survival prediction of } 0 \% \text { or } 100 \% \text { [the } \\
\text { patient can take actions] but with } 45 \% \text { he can't really do } \\
\text { anything. Nor with } 30 \% \text { or } 70 \% \text {." (Clinician } 3 \text { ) }\end{array}$ \\
\hline & $\begin{array}{l}\text { Side-effects are transient and not a basis for } \\
\text { decision-making }\end{array}$ & $\begin{array}{l}\text { "[We wouldn't] say: 'This patient has } 30 \% \text { chance of } \\
\text { dysphagia, so we will do another treatment.' It is a } \\
\text { temporary side-effect and so you also explain it to a } \\
\text { patient. So, to say 'Then we do not do that treatment,' I } \\
\text { think it is not suitable, because it is ultimately a transient } \\
\text { side-effect." (Clinician 2) }\end{array}$ \\
\hline Trust & $\begin{array}{l}\text { Lack of external statistical validation of } \\
\text { CDSS models }\end{array}$ & $\begin{array}{l}\text { "[Models] must naturally be validated on large groups, } \\
\text { and clinical factors must be considered. And even then, } \\
\text { there is still a large variation in a result of such a model. } \\
\text { So yes, it still remains difficult." (Clinician } 8 \text { ) }\end{array}$ \\
\hline $\begin{array}{l}\text { Time } \\
\text { constraints }\end{array}$ & $\begin{array}{l}\text { Additional time and effort needed to use } \\
\text { CDSS in clinical practice }\end{array}$ & $\begin{array}{l}\text { "I think if you have a model in which you have to fill in } 13 \\
\text { variables in order to get a result - that is a hindrance } \\
\text { because it involves too much time and too much work } \\
\text { searching for the data." (Clinician 4) }\end{array}$ \\
\hline
\end{tabular}

\subsection{Discussion}

Decision-making in lung cancer is changing rapidly due to advances in therapies, technologies, and cultural shifts [26,27]. The purpose of this study was to examine treatment decisions in lung cancer both quantitatively in terms of patient deviations and qualitatively by exploring clinician insights, with the aim to determine how CDSSs combined with SDM can support this complex decision-making process. Our study identified the following themes that may influence the introduction and regular use of prognostic CDSSs in clinical practice according to Dutch lung cancer clinicians: a perception that existing clinical guidelines are sufficient to make treatment decisions, lack of trust in existing models due to a lack of large-scale validation studies, and a 
perception that they may not be useful for patients due to difficulties in interpreting risks.

The quantitative part of our study showed that $10.9 \% \quad(n=28)$ of NSCLC patients deviated from MTB advice and nearly 43\% (12 out of 28) of these deviations were due to patient preference for a less intensive treatment. Similar studies find deviation rates ranging from $3 \%$ in esophageal and lung cancer [28] to $8.3 \%$ in neuro-oncological, head and neck, and sarcoma tumors, with patient preference being the biggest reason for deviation (36.5\% of deviations) [29]. However, these findings may underestimate the true level of discordance between patients and clinicians for several reasons. First, the average patient still tends to be heavily influenced by clinician expertise and may expect their clinician to make the final decision [30]. For instance, our qualitative study further confirmed that patient preferences are currently not being taken into account in a systematic manner during consultations. Second, clinician perception that lung cancer patients lack the knowledge or ability to take part in decision-making may hinder their participation [11]. Studies on early stage NSCLC patients find that $49 \%$ preferred a different treatment to the one received [31], 19\% felt insufficiently informed about the benefits and harms of their treatment options, and over $40 \%$ experienced decisional conflict [10]. Third, patients and clinicians differ in how they evaluate clinical information; values clarification experiments suggest that independence and quality of life matter more to lung cancer patients than survival and probability of recurrence [31], while guidelines and MTB discussions tend to base treatment recommendations on the latter and seldom incorporate patient preferences [32-35].

There have been calls to include the patient perspective in MTBs through various means, such as checklists that make note of the patient's preferences, the presence of an oncology nurse who acts as an advocate for the patient, or even having the patient present in the team discussion [36]. While it is desirable to minimize discordance between MTB advice and the administered treatment, particularly in lung cancer management where it is crucial to begin the treatment in 
a timely manner, the short span of time between the first consultation with the pulmonary oncologist and the subsequent MTB can make this impractical. Nevertheless, even though clinical guidelines are an important starting point for decision-making, the fact that nearly half of the deviating patients in our study chose less intensive treatments than the one the MTB advised could be an indication that their preferences need to be taken into account more formally in a SDM process.

Our findings suggest that this SDM talk could take place with the pulmonary oncologist after the MTB. Responses from the clinicians we interviewed revealed a perception that CDSSs are more useful for clinicians than for patients. When implemented in the MTB, a CDSS that generates predicted outcomes and risks of side effects of different treatments for the individual patient can be discussed amongst clinicians and subsequently be used in the consultation to support both clinicians and patients in weighing the trade-off between harms and benefits. According to our results, implementing such a CDSS would require three conditions: externally validation of the underlying prediction model(s), integrated of the CDSS within the hospital's EHR so that the relevant clinical data can be easily accessed, and a combination of risk communication and SDM training for clinicians.

Currently, there is a general lack of lung cancer CDSSs that present the harms and benefits associated with different treatments [15], although certain models have been found to outperform clinicians and guidelines in predicting 2-year survival, dyspnea, and dysphagia [37]. Such models can pave the way for SDM and individualized treatment provided that trust can be built and CDSS outputs can be integrated into consultations. As mentioned previously, CDSSs routinely fail to be adopted in practice [16]. Commonly cited reasons are usability and lack of workflow integration, yet recent evidence suggests that these are secondary to more fundamental issues such as how clinicians view CDSSs [18]. Clinicians in our study cited the lack of external validation as the main barrier. Between $68 \%-75 \%$ of prognostic models are not externally validated $[38,39]$ and many underperform in external 
validation [40]. Measures to standardize statistical validation reporting have been proposed, such as TRIPOD (Transparent Reporting of a multivariable prediction model for Individual Prognosis Or Diagnosis), however adoption of these standards is currently low [41]. The development of privacy-preserving infrastructures to make use of EHR data represents an opportunity to test models across different populations and contexts [39,42]. These developments and our findings highlight the critical need for greater collaboration between researchers, developers, and the academic community in order to harness these innovations [38]. Once developed, CDSSs must be adapted to the local clinical use case through a systematic process [43].

Interpreting CDSS outputs and communicating them to patients in often time-constrained consultations presented a challenge according to our clinicians. SDM relies on two-way communication between patient and clinician, which in turn requires a degree of health literacy [44]. Nearly $10 \%$ of surveyed European adults faced considerable difficulties in interpreting health data and this effect is influenced by educational level and socio-economic background [45]. Measures can be taken to present data in intuitive formats, for instance through visualizations and appropriate framing [46,47]. However, these must be combined with training in empathic communication [48-50], as our findings and prior research confirm the sensitive and emotional nature of lung cancer management [51]. Thus, CDSSs may function as facilitators of communication both among clinicians and between clinicians and patients [52].

\section{Strengths and limitations}

To our knowledge this is the first study that uses qualitative methods to explore Dutch clinicians' perspectives on the decision-making process in lung cancer as well as the place of CDSSs in the workflow and the implications for SDM. One limitation is our study's small sample size due to its exploratory nature; our results represent the views of nine clinicians across six Dutch clinics and may not be generalizable to all contexts. The purpose was to gain deeper insight into the chal- 
lenges associated with implementing a new innovation in lung cancer care, and given the lack of implemented CDSSs in lung cancer as compared to other cancers, this study may function as a precursor to more detailed research into specific use cases. Second, there may be a selection bias in our cohort study as we included only patients referred for radiotherapy; the deviation rate from MTB advice may differ when patients treated with surgery and chemotherapy are included. In addition, the lack of follow-up QoL questionnaires significantly affects the conclusions that can be drawn about the effects of deviating from MTB advice. The results of the retrospective cohort study are therefore meant to function as a starting point for a more detailed investigation into lung cancer patients' treatment choices.

\subsection{Conclusion}

CDSSs have the potential to improve outcomes by introducing patientspecific data in the MTB discussion. Rather than recommending the best treatment option, an ideal CDSS would give an overview of different treatment options, their survival benefit, and impact on quality of life so that these can be used alongside patient preferences in the consultation. Such a system can pave the way for data-driven SDM, in which decisions are based on personalized patient data, patient preferences, and clinician experience. 


\section{Appendix}

Table 5.5 presents the comparison of the survival, recurrence, treatment compliance, toxicities, adverse events, and quality of life between the group of patients who followed MTB advice and those who did not. Differences in survival were calculated with a log-rank test. Chi-squared tests were used to test for differences in recurrence, treatment compliance, toxicities, and adverse events. In order to compare changes in quality of life between the two groups, we used independent t-tests. Due to the low number of events and considerable amount of missing data in the quality of life measures, we can draw some tentative conclusions from our analysis.

There were no significant differences in recurrence, toxicity (dysphagia and dyspnea), treatment compliance, and adverse events related to the treatment between the two groups (recurrence $\mathrm{p}=1.000$; dysphagia $p=0.163$; dyspnea $p=0.557$; treatment compliance $p=0.711$; adverse events $p=0.616$ ) as indicated in Table 5.5. Significant quality of life differences between before and after the treatment were only found with the EQ-5D questionnaire (p-value: 0.043), in which the quality of life was higher in the group who did not follow the advised treatment of the MTB. 
Table 5.5: P-values of the secondary outcomes: survival, recurrence, toxicity

\begin{tabular}{|c|c|c|c|}
\hline & $\begin{array}{l}\text { Patients who did follow the } \\
\text { advice of the tumour board }\end{array}$ & $\begin{array}{l}\text { Patients who did not follow the } \\
\text { advice of the tumour board }\end{array}$ & P-value \\
\hline $\begin{array}{r}\text { Survival } \\
1 \text {-year }\end{array}$ & $\begin{array}{c}\text { Yes:145 } \\
\text { No:84 } \\
\text { Unknown:0 }\end{array}$ & $\begin{array}{c}\text { Yes: } 25 \\
\text { No: } 3 \\
\text { Unknown:0 }\end{array}$ & 0.707 \\
\hline 2-year & $\begin{array}{c}\text { Yes: } 53 \\
\text { No: } 123 \\
\text { Unknown:53 }\end{array}$ & $\begin{array}{c}\text { Yes:12 } \\
\text { No: } 9 \\
\text { Unknown:7 }\end{array}$ & \\
\hline Recurrence & $\begin{array}{c}\text { Yes: } 68 \\
\text { No: } 161 \\
\text { Unknown: } 0\end{array}$ & $\begin{array}{c}\text { Yes: } 8 \\
\text { No: } 20 \\
\text { Unknown: } 0\end{array}$ & 1.000 \\
\hline Below 1-year & $N=52$ & $\mathrm{~N}=7$ & \\
\hline Between 1-2 years & $N=14$ & $\mathrm{~N}=1$ & \\
\hline After 2 years & $\mathrm{N}=2$ & $\mathrm{~N}=0$ & \\
\hline $\begin{array}{l}\text { Toxicity } \\
\text { Dysphagia }\end{array}$ & $\begin{array}{c}\text { Yes: } 122 \\
\text { No: } 107 \\
\text { Unknown: } 0\end{array}$ & $\begin{array}{c}\text { Yes: } 19 \\
\text { No: } 9 \\
\text { Unknown: } 0\end{array}$ & 0.163 \\
\hline Dyspnoea & $\begin{array}{c}\text { Yes: } 30 \\
\text { No: } 199 \\
\text { Unknown: } 0\end{array}$ & $\begin{array}{c}\text { Yes: } 5 \\
\text { No: } 23 \\
\text { Unknown: } 0\end{array}$ & 0.557 \\
\hline $\begin{array}{l}\text { Treatment } \\
\text { compliance }\end{array}$ & $\begin{array}{c}\text { Yes: } 211 \\
\text { No: } 18 \\
\text { Unknown: } 0\end{array}$ & $\begin{array}{c}\text { Yes: } 25 \\
\text { No: } 3 \\
\text { Unknown: } 0\end{array}$ & 0.711 \\
\hline Adverse events & $\begin{array}{c}\text { Yes: } 44 \\
\text { No: } 185 \\
\text { Unknown: } 0\end{array}$ & $\begin{array}{c}\text { Yes: } 7 \\
\text { No: } 21 \\
\text { Unknown: } 0\end{array}$ & 0.616 \\
\hline $\begin{array}{l}\text { Condition } \\
\text { Blood values } \\
\text { Fever } \\
\text { Pain } \\
\text { Other/unknown }\end{array}$ & $\begin{array}{c}N=5 \\
N=15 \\
N=7 \\
N=3 \\
N=14\end{array}$ & $\begin{array}{l}N=3 \\
N=0 \\
N=3 \\
N=1 \\
N=0\end{array}$ & \\
\hline $\begin{array}{l}\text { Quality of life: } \\
E Q-5 D\end{array}$ & $\begin{array}{c}\mathrm{N}=65 \\
\text { Unknown:164 }\end{array}$ & $\begin{array}{c}\mathrm{N}=9 \\
\text { Unknown: } 19\end{array}$ & 0.043 \\
\hline Quality of life: VAS & $\begin{array}{c}\mathrm{N}=93 \\
\text { Unknown: } 136\end{array}$ & $\begin{array}{c}\mathrm{N}=13 \\
\text { Unknown: } 15\end{array}$ & 0.295 \\
\hline $\begin{array}{l}\text { Quality of life: EORTC- } \\
\text { QLQ-C30 }\end{array}$ & $\begin{array}{c}\mathrm{N}=31 \\
\text { Unknown: } 198\end{array}$ & $\begin{array}{c}\mathrm{N}=3 \\
\text { Unknown: } 25\end{array}$ & 0.948 \\
\hline
\end{tabular}




\section{References}

1. Torre LA, Siegel RL, Jemal A. Lung Cancer Statistics [Internet]. In: Ahmad A, Gadgeel S, editors. Lung Cancer and Personalized Medicine. Cham: Springer International Publishing; 2016 [cited 2021 Jan 5]. page 1-19.Available from: http:/ /link.springer.com/10.1007/978-3-319-24223-1_1

2. Zappa C, Mousa SA. Non-small cell lung cancer: current treatment and future advances. Transl. Lung Cancer Res. 2016;5:288-300. 3. Howlader N, Noone A, Krapcho M. National Cancer Institute. SEER Cancer Statistics Review: 1975-2011. 2015;

4. Hirsch FR, Scagliotti GV, Mulshine JL, Kwon R, Curran WJ, Wu Y-L, et al. Lung cancer: current therapies and new targeted treatments. The Lancet 2017;389:299-311.

5. Specchia ML, Frisicale EM, Carini E, Di Pilla A, Cappa D, Barbara A, et al. The impact of tumor board on cancer care: evidence from an umbrella review. BMC Health Serv. Res. 2020;20:73.

6. Stiggelbout AM, Van der Weijden T, De Wit MPT, Frosch D, Légaré F, Montori VM, et al. Shared decision making: really putting patients at the centre of healthcare. BMJ 2012;344:e256.

7. Nemoto K, Murakami M, Ichikawa M, Ohta I, Nomiya T, Yamakawa M, et al. Influence of a multidisciplinary cancer board on treatment decisions. Int. J. Clin. Oncol. 2013;18:574-7.

8. Kostaras X, Shea-Budgell MA, Malcolm E, Easaw JC, Roa W, Hagen NA. Is there a role for clinical practice guidelines in multidisciplinary tumor board meetings? A descriptive study of knowledge transfer between research and practice. J. Cancer Educ. Off. J. Am. Assoc. Cancer Educ. 2012;27:42-5.

9. Ichikawa M, Nemoto K, Miwa M, Ohta I, Nomiya T, Yamakawa M, et al. Status of radiotherapy in a multidisciplinary cancer board. J. Radiat. Res. (Tokyo) 2014;55:305-8.

10. Mokhles S, Nuyttens JJME, de Mol M, Aerts JGJV, Maat APWM, Birim Ö, et al. Treatment selection of early stage non-small cell lung cancer: the role of the patient in clinical decision making. BMC Cancer 2018;18:79.

11. Mokhles S, Maat APWM, Aerts JGJV, Nuyttens JJME, Bogers AJJC, Takkenberg JJM. Opinions of lung cancer clinicians on shared decision 
making in early-stage non-small-cell lung cancer. Interact. Cardiovasc. Thorac. Surg. 2017;25:278-84.

12. Liu Z, Hauskrecht M. A Personalized Predictive Framework for Multivariate Clinical Time Series via Adaptive Model Selection. Proc. ACM Int. Conf. Inf. Knowl. Manag. ACM Int. Conf. Inf. Knowl. Manag. 2017;2017:1169-77.

13. Beauchemin M, Murray MT, Sung L, Hershman DL, Weng C, Schnall R. Clinical decision support for therapeutic decision-making in cancer: A systematic review. Int. J. Med. Inf. 2019;130:103940.

14. De Ruysscher D, van Baardwijk A, Steevens J, Botterweck A, Bosmans $\mathrm{G}$, Reymen B, et al. Individualised isotoxic accelerated radiotherapy and chemotherapy are associated with improved long-term survival of patients with stage III NSCLC: a prospective population-based study. Radiother. Oncol. J. Eur. Soc. Ther. Radiol. Oncol. 2012;102:228-33.

15. Révész D, Engelhardt EG, Tamminga JJ, Schramel FMNH, OnwuteakaPhilipsen BD, van de Garde EMW, et al. Decision support systems for incurable non-small cell lung cancer: a systematic review. BMC Med. Inform. Decis. Mak. 2017;17:144.

16. Yang $Q$, Steinfeld A, Zimmerman J. Unremarkable AI: Fitting Intelligent Decision Support into Critical, Clinical Decision-Making Processes. Proc. 2019 CHI Conf. Hum. Factors Comput. Syst. 2019;1-11.

17. Sittig DF, Wright A, Osheroff JA, Middleton B, Teich JM, Ash JS, et al. Grand challenges in clinical decision support. J. Biomed. Inform. 2008;41:387-92.

18. Liberati EG, Ruggiero F, Galuppo L, Gorli M, González-Lorenzo M, Maraldi $\mathrm{M}$, et al. What hinders the uptake of computerized decision support systems in hospitals? A qualitative study and framework for implementation. Implement. Sci. IS 2017;12:113.

19. Devlin N, Parkin D, Janssen B. An Introduction to EQ-5D Instruments and Their Applications [Internet]. In: Methods for Analysing and Reporting EQ-5D Data. Cham: Springer International Publishing; 2020 [cited 2020 Dec 18]. page 1-22.Available from: http://link.springer.com/10.1007/978-3-03047622-9_1 20. Cline ME, Herman J, Shaw ER, Morton RD. Standardization of the visual analogue scale. Nurs. Res. 1992;41:378-80.

21. Fayers P, Bottomley A, EORTC Quality of Life Group, Quality of Life Unit. 
Quality of life research within the EORTC-the EORTC QLQ-C30. European Organisation for Research and Treatment of Cancer. Eur. J. Cancer Oxf. Engl. 1990 2002;38 Suppl 4:S125-133.

22. Perla RJ, Provost LP. Judgment sampling: a health care improvement perspective. Qual. Manag. Health Care 2012;21:169-75.

23. Braun V, Clarke V. Successful qualitative research: a practical guide for beginners. Los Angeles: SAGE; 2013.

24. McWilliams A, Tammemagi MC, Mayo JR, Roberts H, Liu G, Soghrati K, et al. Probability of Cancer in Pulmonary Nodules Detected on First Screening CT. N. Engl. J. Med. 2013;369:910-9.

25. Herder GJ, van Tinteren H, Golding RP, Kostense PJ, Comans EF, Smit EF, et al. Clinical Prediction Model To Characterize Pulmonary Nodules. Chest 2005;128:2490-6.

26. Gandara DR, Riess JW, Kelly K, Li T, Mack PC, Lara PN. Evolution and Increasing Complexity of the Therapeutic Landscape in Advanced Non-Smallcell Lung Cancer. Clin. Lung Cancer 2017;18:1-4.

27. Gaspar LE, West HJ, Addario BJ, Camidge DR. The patient experience with shared decision-making in lung cancer: A survey of patients, significant others or care givers. Patient Exp. J. 2018;5:50-5.

28. Schmidt HM, Roberts JM, Bodnar AM, Kunz S, Kirtland SH, Koehler RP, et al. Thoracic multidisciplinary tumor board routinely impacts therapeutic plans in patients with lung and esophageal cancer: a prospective cohort study. Ann. Thorac. Surg. 2015;99:1719-24.

29. Hollunder S, Herrlinger U, Zipfel M, Schmolders J, Janzen V, Thiesler T, et al. Cross-sectional increase of adherence to multidisciplinary tumor board decisions. BMC Cancer 2018;18:936.

30. Joseph-Williams N, Elwyn G, Edwards A. Knowledge is not power for patients: A systematic review and thematic synthesis of patient-reported barriers and facilitators to shared decision making. Patient Educ. Couns. 2014;94:291-309.

31. Sullivan DR, Eden KB, Dieckmann NF, Golden SE, Vranas KC, Nugent $\mathrm{SM}$, et al. Understanding patients' values and preferences regarding early stage lung cancer treatment decision making. Lung Cancer 2019;131:47-57. 
32. Gärtner FR, Portielje JE, Langendam M, Hairwassers D, Agoritsas T, Gijsen B, et al. Role of patient preferences in clinical practice guidelines: a multiple methods study using guidelines from oncology as a case. BMJ Open 2019;9:e032483.

33. Rosell L, Alexandersson N, Hagberg O, Nilbert M. Benefits, barriers and opinions on multidisciplinary team meetings: a survey in Swedish cancer care. BMC Health Serv. Res. 2018;18:249.

34. Lamb BW, Brown KF, Nagpal K, Vincent C, Green JSA, Sevdalis N. Quality of care management decisions by multidisciplinary cancer teams: a systematic review. Ann. Surg. Oncol. 2011;18:2116-25.

35. Hahlweg P, Didi S, Kriston L, Härter M, Nestoriuc Y, Scholl I. Process quality of decision-making in multidisciplinary cancer team meetings: a structured observational study. BMC Cancer 2017;17:772.

36. Soukup T, Sevdalis N, Green JSA, Lamb BW, Chapman C, Skolarus TA. Making Tumor Boards More Patient-Centered: Let's Start With the Name. JCO Oncol. Pract. 2021;OP.20.00588.

37. Oberije C, Nalbantov G, Dekker A, Boersma L, Borger J, Reymen B, et al. A prospective study comparing the predictions of doctors versus models for treatment outcome of lung cancer patients: a step toward individualized care and shared decision making. Radiother. Oncol. J. Eur. Soc. Ther. Radiol. Oncol. 2014;112:37-43.

38. Heus P, Damen JAAG, Pajouheshnia R, Scholten RJPM, Reitsma JB, Collins GS, et al. Poor reporting of multivariable prediction model studies: towards a targeted implementation strategy of the TRIPOD statement. BMC Med. 2018;16:120.

39. Riley RD, Ensor J, Snell KIE, Debray TPA, Altman DG, Moons KGM, et al. External validation of clinical prediction models using big datasets from e-health records or IPD meta-analysis: opportunities and challenges. BMJ 2016;i3140.

40. Siontis GCM, Tzoulaki I, Castaldi PJ, Ioannidis JPA. External validation of new risk prediction models is infrequent and reveals worse prognostic discrimination. J. Clin. Epidemiol. 2015;68:25-34.

41. Collins GS, Reitsma JB, Altman DG, Moons K. Transparent reporting of 
a multivariable prediction model for individual prognosis or diagnosis (TRIPOD): the TRIPOD Statement. BMC Med. 2015;13:1.

42. Deist TM, Dankers FJWM, Ojha P, Scott Marshall M, Janssen T, FaivreFinn C, et al. Distributed learning on $20000+$ lung cancer patients - The Personal Health Train. Radiother. Oncol. 2020;144:189-200.

43. Mahadevaiah G, Rv P, Bermejo I, Jaffray D, Dekker A, Wee L. Artificial intelligence-based clinical decision support in modern medical physics: Selection, acceptance, commissioning, and quality assurance. Med. Phys. [Internet] 2020 [cited 2020 Dec 2];47. Available from: https://onlinelibrary.wiley.com/doi/abs/10.1002/mp.13562

44. Stacey D, Hill S, McCaffery K, Boland L, Lewis KB, Horvat L. Shared Decision Making Interventions: Theoretical and Empirical Evidence with Implications for Health Literacy. Stud. Health Technol. Inform. 2017;240:263-83.

45. Sørensen K, Pelikan JM, Röthlin F, Ganahl K, Slonska Z, Doyle G, et al. Health literacy in Europe: comparative results of the European health literacy survey (HLS-EU). Eur. J. Public Health 2015;25:1053-8.

46. Gaissmaier W, Gigerenzer G. Statistical illiteracy undermines informed shared decision making. Z. Für Evidenz Fortbild. Qual. Im Gesundheitswesen 2008;102:411-3.

47. Fagerlin A, Zikmund-Fisher BJ, Ubel PA. Helping patients decide: ten steps to better risk communication. J. Natl. Cancer Inst. 2011;103:1436-43.

48. Johnson Shen M, Ostroff JS, Hamann HA, Haque N, Banerjee SC, McFarland DC, et al. Structured Analysis of Empathic Opportunities and Physician Responses during Lung Cancer Patient-Physician Consultations. J. Health Commun. 2019;24:711-8.

49. Tulsky JA, Arnold RM, Alexander SC, Olsen MK, Jeffreys AS, Rodriguez $\mathrm{KL}$, et al. Enhancing Communication Between Oncologists and Patients With a Computer-Based Training Program: A Randomized Trial. Ann. Intern. Med. 2011;155:593.

50. Fujimori M, Shirai Y, Asai M, Kubota K, Katsumata N, Uchitomi Y. Effect of Communication Skills Training Program for Oncologists Based on Patient Preferences for Communication When Receiving Bad News: A Randomized Controlled Trial. J. Clin. Oncol. 2014;32:2166-72. 
Chapter 5. Clinician perspectives on clinical decision support systems in lung cancer

51. Marlow LAV, Waller J, Wardle J. Does lung cancer attract greater stigma than other cancer types? Lung Cancer 2015;88:104-7.

52. Révész D, Engelhardt EG, Tamminga JJ, Schramel FMNH, OnwuteakaPhilipsen BD, van de Garde EMW, et al. Needs with Regard to Decision Support Systems for Treating Patients with Incurable Non-small Cell Lung Cancer. J. Cancer Educ. Off. J. Am. Assoc. Cancer Educ. 2020;35:345-51. 


\section{Shared decision-making for prophylactic cranial irradiation in extensive-stage small-cell lung cancer: An exploratory study}

Ankolekar, A., De Ruysscher, D., Reymen, B., Houben, R., Dekker, A., Roumen, C., \& Fijten, R.

Translational Lung Cancer Research, 10(7), 3120. 


\section{Abstract}

\section{Background}

Prophylactic cranial irradiation (PCI) offers extensive-stage small-cell lung cancer (ES-SCLC) pa-tients a lower chance of brain metastasis and slightly longer survival but is associated with a short-term decline in quality of life due to side-effects. This tradeoff between survival and quality of life makes PCI suitable for shared decision-making (SDM), where patients and clinicians make treatment decisions together based on clinical evidence and patient preferences. Despite recent clinical practice guidelines recommending SDM for PCI in ES-SCLC, as well as the heavy disease burden, research into SDM for lung cancer has been scarce. This exploratory study presents patients' experiences of the SDM process and decisional conflict for PCI.

\section{Methods}

Radiation oncologists $(n=7)$ trained in SDM applied it in making the PCI decision with ES-SCLC patients $(n=25)$. We measured patients' preferred level of participation (Control Preferences Scale), the level of shared decision-making according to both groups (SDM-Q-9 and SDM-Q-Doc), and patients' decisional conflict (Decisional Conflict Scale).

\section{Results}

Seventy-nine percent of patients preferred a collaborative role in decision-making, and median SDM scores given by patients and clinicians were 80 (IQR: 75.6-91.1) and 85.2 (IQR: 78.7-88.9) respectively, indicating satisfaction with the process. However, many patients experienced considerable decisional conflict. Over $50 \%$ of the patients lacked clarity about which choice was suitable for them and were unsure what to choose. Sixty-four percent felt they did not know enough about the harms and benefits of PCI, and $60 \%$ felt unable to judge the importance of the harms/benefits in their life. 


\section{Conclusions}

ES-SCLC patients prefer to be involved in their treatment choice for PCI but a substantial portion experiences decisional conflict. Better information provision and values clarification may support patients in making a choice that reflects their preferences. 



\subsection{Introduction}

Lung cancer is the leading cause of cancer-related deaths worldwide [1]. Small-cell lung cancer (SCLC) accounts for 15\% of lung cancer cases and has a high mortality rate due to rapid progression and metastases [2]. Prophylactic cranial irradiation (PCI) offers SCLC patients with distant metastases (so-called "extensive disease" patients) a lower chance of developing brain metastasis, a slightly longer overall survival (OS) (median OS from 5.4 months to 6.7 months; 1-year survival rate $27.1 \%$ in the PCI and $13.3 \%$ in the control group), and no long term statistically significant detrimental effect on global health status up to 9 months after treatment [3]. In the short term however, PCI is associated with a decline in quality of life as a result of the treatment, mostly due to fatigue. Recent ESMO clinical practice guidelines call for a shared decision-making (SDM) approach to PCI for ES-SCLC patients [4]. SDM is an interactive process in which patients and clinicians collaborate in choosing treatments based upon the best available evidence and the patient's personal preferences [5].

Treatment decisions such as whether or not to perform PCI are typically made by clinicians from various specializations in a multidisciplinary tumor board, whose recommendation is then communicated to the patient via the treating clinician. In practice, few patients deviate from this recommendation. This is also the case for extensive stage SCLC patients (ES-SCLC), where PCI is considered a standard treatment for patients with adequate performance status after responding to chemotherapy [6]. However, the trade-off between survival and quality of life implies that PCI may be a less attractive option for patients who are expected to have a shorter survival time. Therefore there is a need to take the patient perspective into account [7].

Till date, the SDM process in ES-SCLC has not been explored as the literature tends to focus on preference-sensitive cancer types, such as prostate cancer and breast cancer, while lung cancer decisions tend to be driven by clinical guidelines. As a result, despite the aggressive nature of SCLC and indications of a heavy disease burden in this less 
studied patient population [8], little attention has been paid to how to include patients and their values and preferences in this complex decision. Patients who engage in SDM experience less decisional conflict, improved compliance with treatment, and greater quality of life compared to usual care in various dis-ease areas [9]. Therefore, exploratory work is needed to understand patients' participation desire, experiences, and challenges in the ES-SCLC decision-making process. A better understanding of the patient perspective in the PCI decision may pave the way for adapting the SDM process to this complex context and developing suitable decision support tools.

The purpose of this exploratory study is to determine the extent to which ES-SCLC patients wish to be involved in the PCI decision, the perceived level of SDM when patients and clinicians take this decision together in an SDM process, and the level and sources of decisional conflict these patients experience.

We present the following article in accordance with the MDAR reporting checklist.

\subsection{Methods}

\subsubsection{Participants}

All ES-SCLC patients referred to our clinic (MAASTRO) for PCI who met the following inclusion criteria were invited to participate: (1) WHO performance status $0-2$; (2) no history of prior chest radiotherapy; (3) no other active malignancy; (4) no involvement in another trial which may limit their choice of treatments.

In addition, seven radiation oncologists specialized in lung cancer at MAASTRO took part in this study. These clinicians participated in a one-day workshop covering the fundamentals of SDM theory and practice, specific components of the SDM process, and how to facilitate dialogue with patients at each step. The SDM workshop was designed in collaboration with the Psychology Department of 
the Elisabeth-TweeSteden Hospital (ETZ Tilburg), based on prior experiences with patient focus groups and healthcare professionals. Led by two researchers specialized in SDM, the workshop was participatory in nature; interaction and inputs from the clinicians were encouraged, and these inputs were used to design supplementary materials such as scripts and infographics to facilitate the SDM steps.

\subsubsection{Ethics approval}

This study was conducted in accordance with the Declaration of Helsinki (as revised in 2013). The study protocol was approved by MAASTRO's Internal Review Board (IRB number: P0148) as well as the Academic Hospital Maastricht/Maastricht University's Medical Ethics Review Committee (METC AZM/UM, code: 068, number: 2017-0143.) Informed consent was obtained from all the patients.

\subsubsection{Study procedure}

Patients received an SDM consultation with a trained clinician in order to decide whether or not to undergo PCI. The clinician engaged with the patient in the pre-specified steps of the SDM process, namely acknowledging the decision to make, inviting the patient's participation, informing the patient about PCI and its advantages and disadvantages, eliciting the patient's preferences, and seeking to reach a decision jointly if the patient was prepared to make a decision. After the consultation, clinicians filled in a questionnaire assessing the level of SDM and patients were given a set of questionnaires assessing a variety of SDM measures. Patient questionnaires were administered at three time points: baseline (within one day of the SDM talk), 5 weeks after SDM, and 11 weeks after SDM. 


\subsubsection{Outcome measures}

We assessed the decision-making process using the following questionnaires; control preferences scale, SDM-Q-9/SDM-Q-Doc, and the decisional conflict scale. In addition, patient-reported outcome measures (PROMs) were collected as part of standard procedure. Validated Dutch translations of all questionnaires were used.

\section{Control Preferences Scale (CPS)}

The single-item Control Preferences Scale asks respondents to indicate their desired level of participation in a medical decision by selecting one of five state-ments [10]. The statements range from fully active ("I prefer to make the final treatment decision") to fully passive ("I prefer to leave all treatment decisions to my doctor"), with the midpoint being a collaborative role ("I prefer that my doctor and I share responsibility for deciding which treatment is the best").

\section{SDM-Q-9 (patients) and SDM-Q-Doc (clinicians)}

The SDM-Q-9 and SDM-Q-Doc instruments measure patients' and clinicians' assessment of the level of patient involvement in the decision-making process [11]. The instrument consists of nine items covering the steps of the SDM process, which can be rated on a six-point scale from 0 (completely disagree) to 5 (completely agree). Patients' and clinicians' raw scores were summed up and rescaled to be on a $0-100$ range to be comparable [12]. In this study, the patients received the SDM-Q-9 with a five-point Likert scale, whereas the clinicians received the SDM-Q-Doc with the original six-point Likert scale. As a result, the patient version has a neutral point ('No opinion'), whereas the clinician version induces the respondent to choose between 'Somewhat disagree' and 'Somewhat agree'. Raw scores have been presented for individual item responses to compare between patients and clinicians.

\section{Decisional Conflict Scale (DCS)}

The decisional conflict scale asks patients to report the level of un- 
certainty they experienced while making their decision using a fivepoint Likert scale [13]. This 16-item scale has five subscales: feeling informed, decisional uncertainty, clear values, support, and quality of decisions. Scoring the DCS results in a scale ranging from 0 (no decisional conflict) to 100 (extremely high decisional conflict). Cutoff points are 0-25 (low decisional conflict, high level of decision certainty), 25-37.5 (moderate level of decisional conflict), and above 37.5 (significant uncertainty about decision).

\section{Patient-reported outcome measures (PROMs)}

Standard PROMs were collected at all three time points. The EORTC QLQ-C30 measures quality of life of cancer patients along physical, psychological, and social dimensions [14]. Finally, the EORTC QLQBN20 measures the quality of life of patients receiving treatment for brain tumors along several dimensions, such as future uncertainty, visual disorder, motor dysfunction, and communication deficit [15].

\subsubsection{Statistical analysis}

The data from completed questionnaires was scored according to the respective questionnaire's scoring guide. Data analysis was conducted using IBM SPSS. Initial descriptive analysis revealed that SDM-Q-9, SDM-Q-Doc, DCS scores and quality of life scores were not normally distributed, therefore non-parametric tests were used. Patient and clinician SDM scores were compared using a Mann-Whitney U test. Spearman's correlation coefficients were calculated to determine the relationship between SDM and DCS. Wilcoxon signed rank tests were used to compare DCS scores over time.

\subsection{Results}

Between December 2017 and December 2019, 31 patients consented to take part in the study. Following the SDM talk, the majority of patients $(84 \%)$ chose to undergo PCI, while five patients $(16 \%)$ chose not to. The median survival time of all patients was 26.6 weeks (SD: 20.2). 
Twenty-five patients returned baseline questionnaires, yielding a completion rate of $80.6 \%$. Out of these 25 patients, 18 returned the 5 -week follow-up questionnaires and 15 returned the 11-week follow-up questionnaires. Two patients were deceased by the time of their 11-week follow-up but only four patients remained alive at the closing date of the study (May 2020), indicating that disease progression could be a major factor in loss to follow-up.

Characteristics of the 25 baseline patients and the seven participating clinicians are presented in Table 6.1. The patient sample was roughly evenly split across gender and the mean age was 66.8 years (SD: 8.2). TNM staging was indicative of extensive stage SCLC, with the majority of patients having metastasis to the lymph nodes and distant metastasis. The majority of patients had a performance status of 1 or lower, indicating an ability to carry out light activities; the remaining $12 \%$ of patients had a performance status of 2 indicating ability to perform self-care but not work activities.

\subsubsection{Patients' preferred level of involvement in decision-making}

The majority of patients (79\%) preferred to play an active or collaborative role in their treatment decision, as measured by the CPS (Figure 6.1). Of these, one patient preferred to make the decision alone, while the remaining patients preferred to share the decision-making role with their clinician to varying degrees. Five patients (approximately $20 \%$ ) preferred a more passive role in which the clinician makes the final decision. These findings remained fairly consistent across the three measurement points, suggesting that patients' experience of their treatment choice did not change their preferred role in the decisionmaking process. 
Table 6.1: Demographic and clinical characteristics of patients and clinicians who participated in the study

\begin{tabular}{llll}
\hline Patients & $\mathbf{n}(\%)$ & Clinicians & $\mathbf{n}(\%)$ \\
\hline Total & 25 & Total & 7 \\
$\quad$ Female & $12(48)$ & Female & $4(57)$ \\
$\quad$ Male & $13(52)$ & $\begin{array}{c}\text { Male } \\
\text { Age (years) }\end{array}$ & $3(43)$ \\
Age (years) & & $30-39$ & $3(43)$ \\
$40-49$ & $1(4)$ & $40-49$ & $2(29)$ \\
$50-59$ & $3(12)$ & $50-59$ & $1(14)$ \\
$60-69$ & $10(40)$ & $60-69$ & $1(14)$ \\
$70-79$ & $10(40)$ & Experience (years) & \\
$80+$ & $1(4)$ & $0-5$ & $1(14)$ \\
N-stage & & $6-10$ & $2(29)$ \\
cN0-cN2 & $8(32)$ & $11-15$ & $1(14)$ \\
cN3 & $17(68)$ & $16-20$ & $1(14)$ \\
M-stage & & $20+$ & $2(29)$ \\
M1a or M1b solitary & $4(16)$ & & \\
M1b (other) & $21(84)$ & & \\
WHO performance & & & \\
status & & & \\
0 & $4(15)$ & & \\
1 & $18(72)$ & & \\
2 & $3(12)$ & &
\end{tabular}

\subsubsection{The level of SDM perceived by patients and clinicians}

With 100 representing the highest level of SDM, the median SDM score at baseline was 80 (IQR: 75.6-91.1) according to patients and 85.2 (IQR: 78.7-88.9) according to clinicians (Figure 6.2). There was a higher variability in patient SDM scores, however a Mann-Whitney $U$ test showed no significant difference in median SDM scores between patients and clinicians as a whole ( $\mathrm{z}$-score $-0.514, \mathrm{p}=0.610$ ). Spearman's rho correlation between matching patient-clinician pairs indicated no significant pairwise correlation ( $\mathrm{rs}=0.287, \mathrm{p}=0.164$ ).

These results indicate that patients and clinicians did not differ significantly as a group in their estimation of the SDM level, however their 


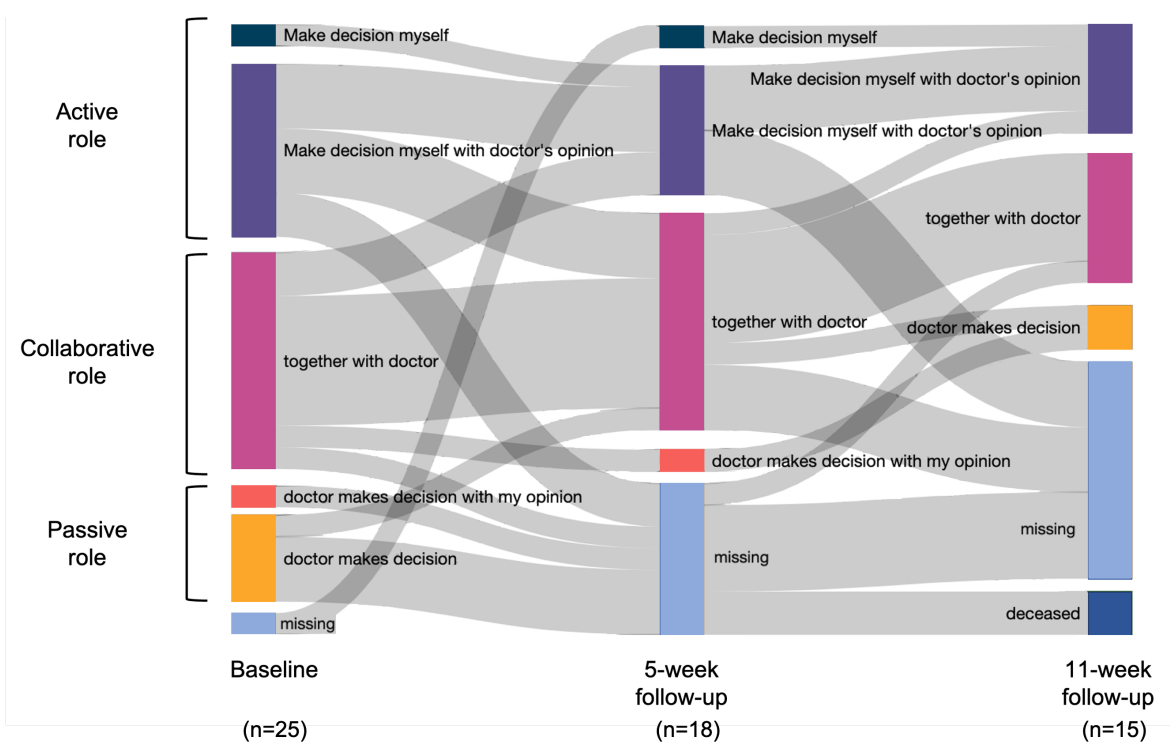

Figure 6.1: Control Preference Scale measurements showing patients' preferred level of participation in the decision, ranging between active ("I prefer to make the decision myself" or "I prefer to make the decision myself with the doctor's opinion"), collaborative ("I prefer to make the decision together with the doctor"), and passive ("I prefer that the doctor makes the final decision with my opinion considered" or "I prefer that the doctor makes the decision entirely").

pairwise agreement was low. To investigate this further, we compared patient and clinician responses on individual items of the SDM-Q-9 and SDM-Q-Doc questionnaire (Figure 6.3).

Patients and clinicians were in agreement that it was sufficiently clear that a treatment decision needs to be made (Item 1) and that corresponding information were adequately explained (Items 4 and 5). Most of the patients ( $84 \%$ ) felt that their clinician wanted to know to what extent the patient wishes to be involved in decision-making, and an equal percentage of clinicians reported the same (Item 2). 


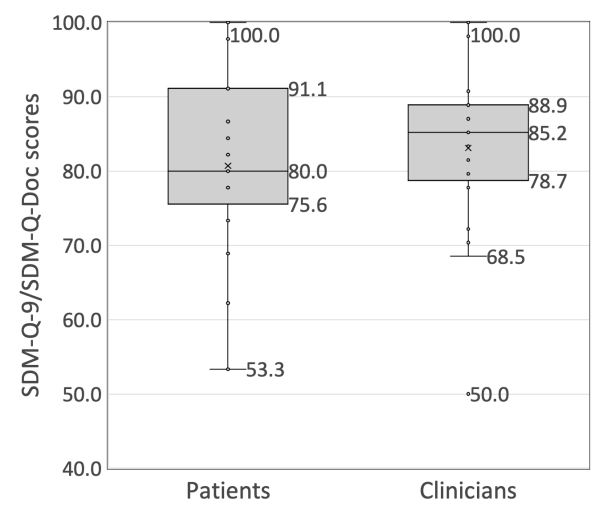

Figure 6.2: Patients' and clinicians' perceptions of the SDM process at baseline. The vertical axis represents SDM scores rescaled to a 0-100 scale, with 0 (not shown) indicating no SDM and 100 representing the highest level of SDM. Responses ranged from 50-100, with median 80 (patients) and 85.2 (clinicians).

Patients and clinicians differed on whether the treatment options were presented adequately (Item 3), with more patients disagreeing on this statement. There was also disagreement on the extent to which the patient's treatment preference was explicitly sought (Item 6); all clinicians agreed to varying degrees that they asked the patient which treatment option he/she prefers, while only $68 \%$ of patients agreed with the same statement. Finally, we observed a discrepancy between patients and clinicians regarding whether the treatment options were thoroughly weighed (Item 7). Here again, patients disagreed with these statements more than clinicians.

Spearman's correlation between patient SDM scores and their CPS scores was positive and statistically significant ( $r s=0.501, p=0.013$ ), indicating that patients with higher SDM scores tended to prefer a more shared decision process with their clinician. 


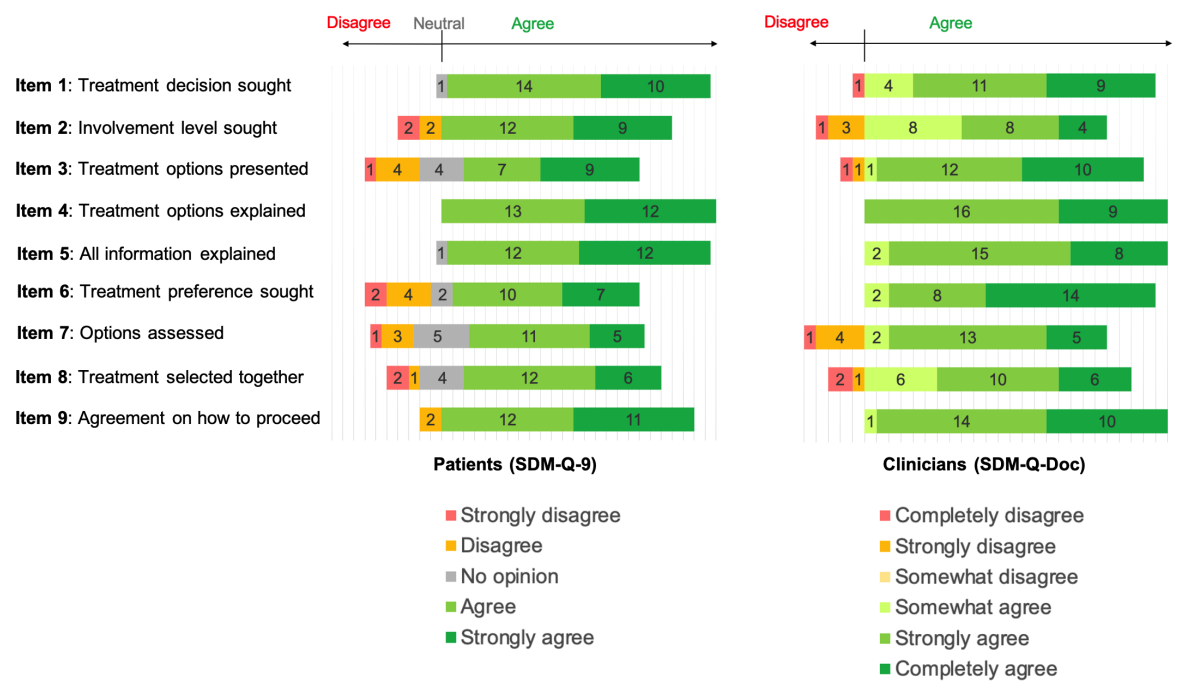

Figure 6.3: A comparison of the item scores on the SDM-Q-9 questionnaire from 25 patients and the SDM-Q-Doc questionnaire from seven clinicians who participated in the consultations of these 25 patients. The vertical axis displays the individual items in questionnaire and the horizontal axis represents the level of agreement with the items. The scales between patients and clinicians differed, namely patients answered on a five-point Likert scale with a neutral mid-point ('No opinion') whereas clinicians answered on a six-point Likert scale with no neutral mid-point.

\subsubsection{The extent of decisional conflict perceived by patients}

The level of decisional conflict patients experienced with making the choice whether to undergo PCI is presented in Figure 6.4. The median decisional conflict score for patients at baseline was 21.9 (IQR: 3.1-25) out of 100 , and nine patients $(36 \%)$ reported experiencing decisional conflict at baseline. The level of decisional conflict rose in the weeks following the decisional talk: five weeks and 11 weeks after baseline the median DCS was 17.2 (IQR: 3.9-32.4) and 25 (IQR: 14.1-28.1) respectively. DCS scores were not significantly correlated with patients' age $(\mathrm{rs}=0.086, \mathrm{p}=0.681)$. Wilcoxon signed rank tests showed no signifi- 
cant difference in median paired DCS scores from baseline to 5-week follow-up and from 5-week to 11-week follow-up.

The three most significant sources of decisional conflict for patients were uncertainty, not feeling sufficiently informed, and a lack of clarity about their own personal values (Figure 6.5). Patients experienced the most severe decisional conflict on the uncertainty subscale; over $50 \%$ reported a lack of clarity about which choice is best, feeling unsure about what to choose, and that the decision was difficult to make. For $20 \%$ of patients this feeling was pronounced enough for them to feel that they might not have made the right decision. Most patients (64\%) also experienced moderate to severe decisional conflict on the informed subscale, indicating a self-reported lack of awareness about the potential benefits and risks of PCI. Subsequently, $60 \%$ of patients also reported not being able to judge how important the benefits and harms would be to them, as indicated by the values clarity subscale.

Patients' perceived level of SDM was significantly inversely correlated with their level of decisional conflict ( $r s=-0.507, p=0.01$ ). In other words, patients who rated the level of SDM as higher tended to report having lower levels of decisional conflict. There was no statistically significant correlation between clinicians' SDM scores and patients' decisional conflict $(\mathrm{rs}=0.106, \mathrm{p}=0.614)$. 


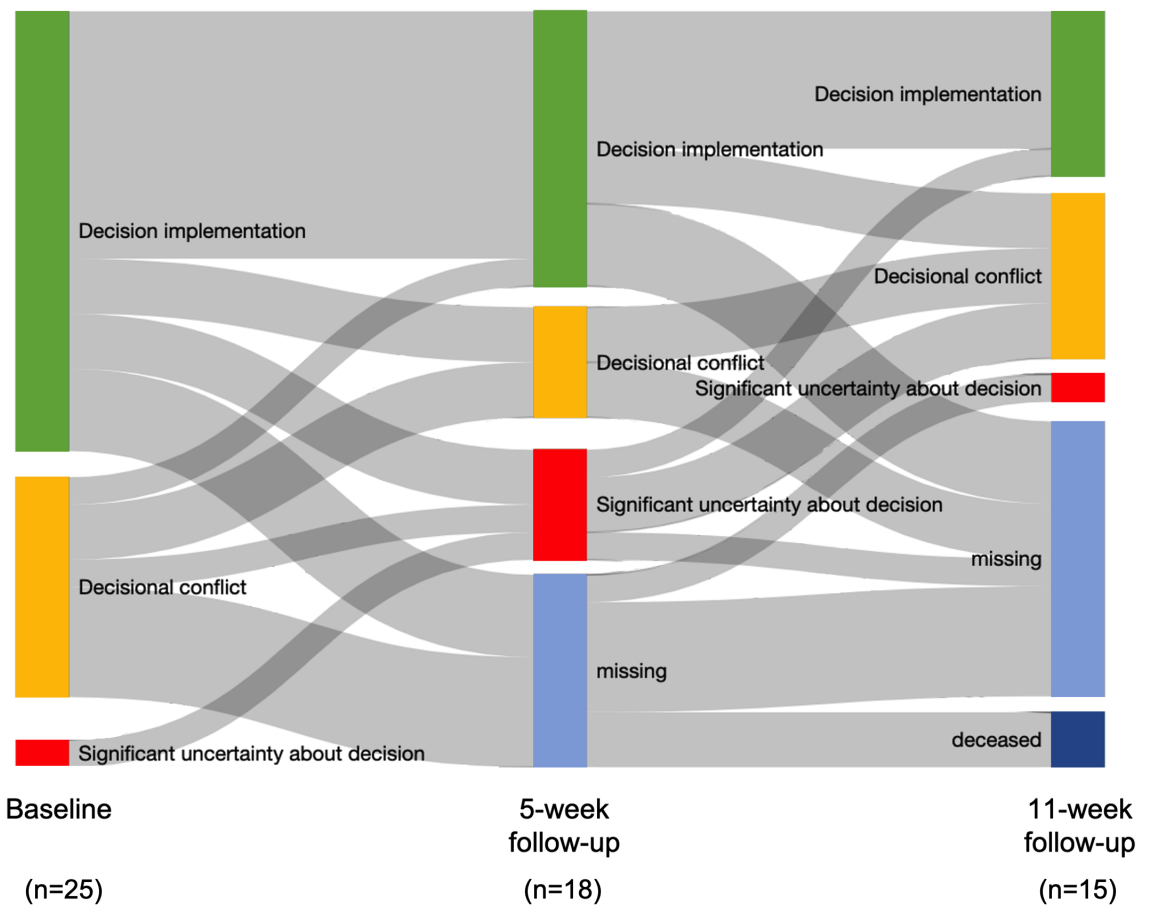

Figure 6.4: Patients' decisional conflict levels measured by the Decisional Conflict Scale at baseline and 5-week and 11-week follow-ups 'Decision implementation' (green bar) indicates that that patients experienced little to no conflict in making their choice. 'Decisional conflict' (yellow bar) refers to a moderate level of decisional conflict, and 'Significant uncertainty about decision' (red bar) indicates severe difficulty in making the treatment decision and doubt whether the best decision was made. Missing data and deceased patients are indicated by light blue and dark blue bars respectively. 


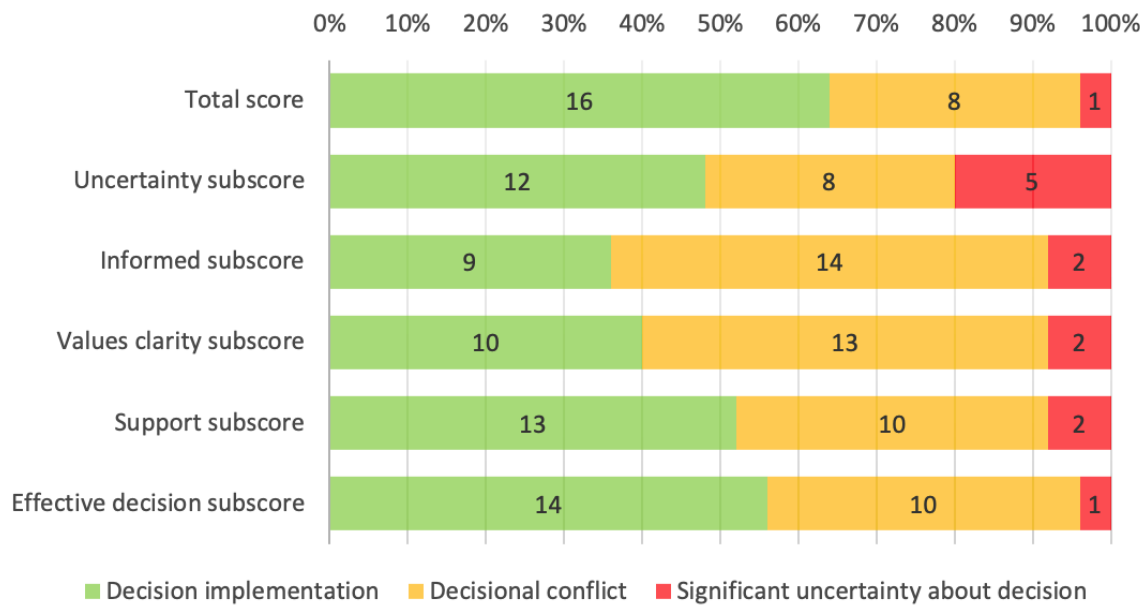

Figure 6.5: A breakdown of the decisional conflict subscales at baseline. "Decision implementation" indicates that patients faced little to no decisional conflict. Decision implementation' (green bar) indicates that that patients experienced little to no conflict in making their choice. 'Decisional conflict' (yellow bar) refers to a moderate level of decisional conflict, and 'Significant uncertainty about decision' (red bar) indicates severe difficulty in making the treatment decision. 


\subsection{Discussion}

PCI is currently given as a standard treatment to ES-SCLC patients as a way to limit the development of brain metastases. However, it can lead to severe short-term side-effects such as cognitive decline and fatigue $[16,17]$. This raises the question of whether PCI is preferable for patients who may have a poor survival prognosis. This dilemma is exemplified by a median survival of around 6 months in our study population, which is insufficient time to reap the long-term benefits of PCI. The median OS in the present study is similar to that in the EORTC study by Slotman et al. on which the current guidelines are based [3]. This, and other recent work, highlights the need to take patient preference into consideration for this decision through an SDM process [17].

In this exploratory study we implemented a simple SDM model and explored the level of SDM and decisional conflict perceived by ESSCLC patients and their clinicians in making the decision whether to undergo PCI to reduce the risk of brain metastases. A sizable portion of patients in our sample $(16 \%)$ chose not to undergo the guidelinerecommended PCI. This deviation is high-er than previously observed in our clinic since PCI has been offered as the standard treatment to ES-SCLC patients, however due to a small sample size our study is not sufficiently powered to establish the statistical significance of this finding. Nevertheless, our study offers insights into patients' experience of the decision-making process and how it may be improved.

\subsubsection{Patient participation and preferences for SDM}

We found that patients and clinicians rated the level of SDM highly, though there was no significant pairwise correlation between patient SDM scores and clinician SDM scores. Indeed, we observed discrepancies between patients and clinicians, for instance, while all the clinicians agreed to varying degrees that they had asked their patient's preference, a sizable portion of patients disagreed. Such discrepancies have been observed in prior research through independent observa- 
tion using the OPTION scale, a validated 12-item instrument that measures the extent to which a clinician involves a patient in the decisionmaking process [18]. These findings reveal that although many clinicians may feel that they practice SDM, the actual level of SDM might not be as high as they perceived [19]. This may be due to varying definitions of what constitutes SDM, for instance, some clinicians conflate it with informed decision-making [20]. Our results and these previous findings suggest that when designing SDM training, certain as-pects of the SDM process may require more attention and emphasis. In particular, active engagement through role-play may help clarify the difference between informed decision-making and SDM [21]. Evaluation of SDM implementation could also include the OPTION scale as an objective measure of the effectiveness of SDM training.

The majority of patients in our sample preferred a collaborative role in the decision-making process, with only a small portion preferring a completely passive role in which the clinician chooses the treatment and even fewer preferring a completely active role with no input from the clinician. This confirms prior research that finds a similar distribution of participation preference among lung cancer patients [22,23]. We also found a correlation between CPS and SDM, suggesting that patients who preferred a more active role were more ready to engage in the SDM process and consequently gave a higher score on the SDMQ9. An alternative interpretation could be that having undergone an SDM process, patients found that they would prefer to be involved in their medical decisions in general, and their CPS scores reflected this. In either case, the discrepancies observed between patient and clinician SDM scores suggest that while clinicians are successfully able to engage patients in making choices, more effort may be needed to help them discover how their treatment choices may impact their quality of life. In other words, the clinician's role should not be to give patients all the relevant information and let them decide, but to take an active role in supporting the patient for truly shared decisions. 


\subsubsection{Decisional conflict levels}

We found that $36 \%$ of patients experienced decisional conflict when deciding whether to undergo PCI, mainly due to the feeling of not understanding the benefits, risks, or side-effects of PCI, and lack of clarity about which option was best for them. Similar findings have been reported in the Dutch and Korean contexts [22,24]. For instance, the former study finds that $40 \%$ of early-stage NSCLC patients experience decisional conflict, mostly with respect to the informed and uncertainty subscales. In lung cancer, it seems that treatment options and outcomes are often hard to grasp for non-experts and that there often are no treatment options with desired outcomes, especially given the stage of the disease that we studied. Future work should find ways to inform patients better, but it is likely these patients will continue to experience a high level of decisional conflict until better treatment options become available for this disease.

Our finding of a significant correlation between SDM and DCS scores suggests that when patients feel involved in the decision-making process, they experience lower decisional conflict. This relationship conforms with similar studies $[25,26]$. Taken together, these findings underline the importance of SDM for the PCI decision and suggest that the SDM process should place significant emphasis on deliberating on the risks and benefits of the treatment options and what they may imply for individual patients. Prior research has shown that access to accurate clinical information improves people's ability to manage decisional uncertainty [27].

\subsubsection{Strengths and limitations}

It is important to bear in mind some limitations of our study design. First, the sample size of our study (25 patients) limits the generalizability of our results. The ES-SCLC patient group is a small sub-population of lung cancer patients and recruiting a sufficiently powered sample is a challenge. Previous landmark studies on PCI for ES-SCLC patients have required a large number of centers in order to obtain suf- 
ficiently powered samples $[28,29]$. Our ability to extend across multiple centers was limited by our goal of implementing SDM training, as designing and conducting such trainings requires considerable resources and coordination. Challenges in data collection were exacerbated by the rapid progression of the disease, which was a possible reason for the missing data in our sample; out of 25 patients, seven were lost to follow-up by the 5-week mark and 12 by the 11-week mark, of which two patients were deceased. This affects the strength of our conclusions, particularly where statistical significance could not be established. On a related note, a second limitation is that we were not able to include a control group of ES-SCLC patients who did not undergo an SDM talk for PCI due to the limited number of patients treated in this clinical use-case. Consequently, we were unable to determine the base-line level of decisional conflict for usual care ES-SCLC patients. Our findings could be strengthened by conducting a multicenter trial to generate a larger sample. In addition, we explore patient and clinician perceptions of the level and process of SDM; we were unable to conduct independent observations of the level of SDM, such as through the OPTION scale. An additional objective measurement may provide deeper insights into the extent of patient participation, particularly since we found discordance between patients' and clinicians' perceptions on certain aspects of the SDM-Q-9/SDM-Q-Doc questionnaires.

Notwithstanding these limitations, this study adds to our understanding of the decision-making process in ES-SCLC, a disease area that has not been adequately explored in the context of SDM. Indeed, SCLC has been referred to as an orphan disease due to its low prevalence [30], yet its aggressive nature and high economic burden warrant a deeper exploration of the patient perspective [8]. Although our data is limited, our study may function as a starting point for discussion in the medical community about how best to involve patients in this value-laden decision, particularly in light of upcoming ESMO clinical practice guidelines that recommend SDM for the PCI choice for ES-SCLC patients [4]. Little is known about patient preferences regarding PCI. Discrete 
choice experiments for PCI with stage III NSCLC patients suggest that survival benefit is the most important consideration for that patient population [31]. Patients were willing to tolerate a significant degree of toxicity if it meant greater survival benefit, and as potential survival benefits decreased so did the level of toxicity the patients were willing to accept. In particular, patients were willing to trade-off potential memory loss and their ability for self-care for a decrease in likelihood of brain metastases.

Future work could use PROMs to explore more extensively the specific dimensions of quality of life that matter to PCI patients in order to guide SDM consultations. For instance, changes in quality of life measures in the weeks following PCI may provide some indication as to which side-effects and toxicities decline most severely. This may assist clinicians in focusing the SDM talk and provide a basis for asking patients about what matters to them. Future research could also focus on developing clinical decision support tools which present individualized risk predictions for PCI based on PROMs [32]. This may be useful in light of our findings and those of NSCLC in the curative setting [22] that show that one of the biggest sources of decisional conflict is patients' difficulties in balancing the harms and benefits of their treatment options.

To conclude, the added value of PCI for ES-SCLC patients is still being debated in the clinical community [33]. This study shows that patients who are engaged in SDM on whether to undergo PCI feel sufficiently involved in the decision-making process, but a considerable portion still experience decisional conflict, particularly in understanding the benefits and risks of the treatment. This leads to feelings of uncertainty about whether they made the best choice. While it may not be possible to eliminate decisional conflict entirely, clinical tools such as prognostic models may provide a clearer picture of the added benefit of PCI in the individual patient. Such tools must be combined with SDM with an emphasis on risk communication so that ES-SCLC patients can make a choice based on their preferences and the best available evidence. 


\section{Appendix}

Standard patient questionnaires collected as part of patient-reported outcomes (PROMs) included:

EORTC QLQ-C30: A questionnaire developed to assess the quality of life of cancer patients. The QLQ-C30 is composed of both multiitem scales and single-item measures. These include a global health status/QoL scale, five functional scales, three symptom scales, and six single items. All of the scales and single-item measures range in score from 0 to 100. For global health status and functional scales, a higher score indicates better QoL. For symptom scales, a higher score indicates worse symptoms.

EORTC QLQ-BN20: A specialized questionnaire developed to assess the quality of life of patients undergoing chemotherapy or radiotherapy for brain tumors. It includes 20 items with four multi-item scales (future uncertainty, visual disorder, motor dysfunction, and communication deficit), and single-item symptom scales (e.g. headaches and seizures). When the questionnaire is scored, all scales and items range from 0 to 100, with a higher score representing worse symptoms.

\section{EORTC QLQ-C30}

EORTC QLQ-C30 scores at baseline and follow-up are presented in Table 6.2. We observe a moderate level of QoL at baseline (median score: 66.7, IQR: 58.3-83.3) that remained relatively constant over follow-up with a slight increase in median score 11 weeks after SDM (median score: 75.0, IQR: 54.2-83.3). On functional scales, patients reported the highest scores on the cognitive and social domains, though the median social functioning score declined in the 5 weeks following SDM from 100 (IQR: 83.3-100) to 66.7 (IQR: 50.0-83.3). Role functioning had the lowest median score throughout the observation period.

Fatigue and dyspnea were the most commonly reported symptoms at baseline, with a median score of 33.3 (IQR: 22.2-44.4 and 0.0-33.3 respectively). Median scores for appetite loss, pain, and nausea rose 
from 0.0 at baseline to 33.3 (IQR: 0.0-66.7), 25.0 (IQR: 0.0-33.3), and 16.7 (IQR: 0.0-50.0) at the first follow-up, and remained at these levels with the exception of pain which declined slightly to 16.7 (IQR: 0.033.3). These values are comparable with European references values for ES-SCLC patients presented in the EORTC QLQ-C30 reference values manual.

Table 6.2: Patients' median EORTC QLQ-C30 scores at baseline, 5-week follow-up, and 11-week follow-up. Interquartile ranges are reported in parentheses. Scores range from 0-100. For global health status and the five functional scales, a score of 100 indicates the best quality of life. For symptom scales, a score of 100 indicates the worst symptoms.

\begin{tabular}{lccc}
\hline EORTC QLQ-C30 (median, IQR) & $\begin{array}{c}\text { Baseline } \\
(\mathbf{n = 2 5})\end{array}$ & $\begin{array}{c}\text { 5-week follow-up } \\
(\mathbf{n = 1 8})\end{array}$ & $\begin{array}{c}\text { 11-week follow-up } \\
\text { (n=15) }\end{array}$ \\
\hline Global health status/QoL & $66.7(58.3-83.3)$ & $66.7(50.0-81.3)$ & $75.0(54.2-83.3)$ \\
& & & \\
Functional scales & & & \\
Physical functioning & $75.0(60.0-80.0)$ & $60.0(40.0-73.3)^{+}$ & $73.3(46.7-80.0)$ \\
Role functioning & $66.7(66.7-83.3)$ & $66.7(33.3-66.7)$ & $66.7(50.0-83.3)$ \\
Emotional functioning & $75.0(66.7-91.7)$ & $79.2(66.7-91.7)$ & $83.3(66.7-95.8)$ \\
Cognitive functioning & $100.0(83.3-100.0)$ & $83.3(66.7-95.8)$ & $83.3(66.7-100.0)$ \\
Social functioning & $100.0(83.3-100.0)$ & $66.7(50.0-83.3)$ & $83.3(66.7-100.0)$ \\
& & & \\
Symptom scales & & & \\
Fatigue & $33.3(22.2-44.4)$ & $44.4(44.4-88.9)$ & $44.4(33.3-80.6)$ \\
Nausea & $0.0(0.0-0.0)$ & $16.7(0.0-50.0)$ & $16.7(0.0-33.3)$ \\
Pain & $0.0(0.0-33.3)$ & $25.0(0.0-33.3)$ & $16.7(0.0-33.3)$ \\
Dyspnea & $33.3(0.0-33.3)$ & $33.3(33.3-66.7)$ & $33.3(33.3-66.7)$ \\
Insomnia & $0.0(0.0-33.3)$ & $33.3(8.3-66.7)$ & $33.3(0.0-50.0)$ \\
Appetite loss & $0.0(0.0-33.3)$ & $33.3(0.0-66.7)$ & $33.3(0.0-66.7)$ \\
Constipation & $0.0(0.0-0.0)$ & $0.0(0.0-0.0)$ & $0.0(0.0-33.3)$ \\
Diarrhea & $0.0(0.0-0.0)$ & $0.0(0.0-33.3)$ & $0.0(0.0-33.3)$ \\
Financial difficulties & $0.0(0.0-0.0)$ & $0.0(0.0-33.3)$ & $0.0(0.0-16.7)$ \\
\hline
\end{tabular}

tBased on 17 observations due to one missing data point.

\section{EORTC QLQ-BN20}

Table 6.3 shows that of the four multi-item scales, patients reported the highest levels on future uncertainty at baseline, with a median 
score of 33.3 and IQR 22.2-44. Of the symptom scales, hair loss was the most severe symptom; the median score of 66.7 at baseline rose to 100.0 (IQR: 66.7-100) five weeks after SDM. This may be related to chemotherapy treatments prior to making the PCI decision. Similarly, headaches, drowsiness, and itchy skin were reported to be most severe at the 5-week mark and declined by the 11-week mark.

Table 6.3: Patients' median EORTC QLQ-BN20 scores at baseline, 5-week follow-up, and 11-week follow-up. Interquartile ranges are reported in parentheses. Scores range from $0-100$, with 100 representing the worst quality of life/symptoms.

\begin{tabular}{|c|c|c|c|}
\hline EORTC QLQ-BN20 (median, IQR) & $\begin{array}{l}\text { Baseline } \\
\qquad(n=25)\end{array}$ & $\begin{array}{l}\text { 5-week follow-up } \\
\qquad(n=18)\end{array}$ & $\begin{array}{l}\text { 11-week follow-up } \\
\qquad(n=15)\end{array}$ \\
\hline \multicolumn{4}{|l|}{ Scales } \\
\hline Future uncertainty & $33.3(22.2-44.4)$ & $38.9(22.2-63.9)$ & $22.2(11.1-44.4)$ \\
\hline Visual disorder & $0.0(0.0-11.1)$ & $16.7(0.0-33.3)$ & $11.1(0.0-22.2)$ \\
\hline Motor dysfunction & $0.0(0.0-11.1)$ & $11.1(0.0-33.3)$ & $11.1(0.0-22.2)$ \\
\hline Communication deficit & $0.0(0.0-0.0)$ & $0.0(0.0-11.1)$ & $0.0(0.0-22.2)$ \\
\hline \multicolumn{4}{|l|}{ Symptom scales } \\
\hline Headaches & $0.0(0.0-0.0)$ & $33.3(8.3-33.3)$ & $0.0(0.0-33.3)$ \\
\hline Seizures & $0.0(0.0-0.0)$ & $0.0(0.0-0.0)$ & $0.0(0.0-0.0)$ \\
\hline Drowsiness & $0.0(0.0-33.3)$ & $33.3(8.3-66.7)$ & $33.3(0.0-50.0)$ \\
\hline Hair loss & $66.7(0.0-100.0)$ & $100.0(66.7-100.0)^{\dagger}$ & $66.7(16.7-100.0)$ \\
\hline Itchy skin & $0.0(0.0-33.3)$ & $33.3(0.0-66.7)^{\dagger}$ & $0.0(0.0-33.3)^{\ddagger}$ \\
\hline Weakness of legs & $0.0(0.0-33.3)$ & $33.3(0.0-33.3)^{\dagger}$ & $0.0(0.0-33.3)$ \\
\hline Bladder control & $0.0(0.0-33.3)$ & $0.0(0.0-33.3)^{\dagger}$ & $0.0(0.0-0.0))$ \\
\hline
\end{tabular}




\section{References}

1. Torre LA, Siegel RL, Jemal A. Lung Cancer Statistics. Adv Exp Med Biol 2016;893:1-19.

2. Huber RM, Tufman A. Update on small cell lung cancer management. Breathe 2012;8:314-30.

3. Slotman BJ, Mauer ME, Bottomley A, et al. Prophylactic cranial irradiation in extensive disease small-cell lung cancer: short-term health-related quality of life and patient reported symptoms-results of an international phase III randomized controlled trial by the EORTC radiation oncology and lung cancer groups. J Clin Oncol 2009;27:78-84.

4. Dingemans A-MC, Früh M, Ardizzoni A, et al. Small-cell lung cancer: ESMO Clinical Practice Guidelines for diagnosis, treatment and follow-up. Ann. Oncol. 2021;S0923-7534(21)01113-3.

5. Elwyn G, Frosch D, Thomson R, et al. Shared decision making: a model for clinical practice. J Gen Intern Med 2012;27:1361-7.

6. Vansteenkiste J, De Ruysscher D, Eberhardt WEE, et al. Early and locally advanced non-small-cell lung cancer (NSCLC): ESMO Clinical Practice Guidelines for diagnosis, treatment and follow-up. Ann Oncol Off J Eur Soc Med Oncol 2013;24 Suppl 6:vi89-98.

7. Johnson SB, Decker RH. Prophylactic Cranial Irradiation Versus Surveillance: Physician Bias and Patient-centered Decision-making. Clin Lung Cancer 2018;19:464-6.

8. Enstone A, Greaney M, Povsic M, et al. The Economic Burden of Small Cell Lung Cancer: A Systematic Review of the Literature. PharmacoEconomics 2018;2:125-39.

9. Stacey D, Légaré F, Lewis $\mathrm{K}$, et al. Decision aids for people facing health treatment or screening decisions. Cochrane Database Syst Rev 2017;4:CD001431.

10. Degner LF, Sloan JA, Venkatesh P. The control preferences scale. Can J Nurs Res 1997;21-43.

11. Kriston L, Scholl I, Hölzel L, et al. The 9-item Shared Decision Making Questionnaire (SDM-Q-9). Development and psychometric properties in a primary care sample. Patient Educ Couns 2010;80:94-9. 
12. Rodenburg-Vandenbussche S, Pieterse AH, Kroonenberg PM, et al. Dutch Translation and Psychometric Testing of the 9-Item Shared Decision Making Questionnaire (SDM-Q-9) and Shared Decision Making QuestionnairePhysician Version (SDM-Q-Doc) in Primary and Secondary Care. PLOS ONE 2015;10:e0132158.

13. O'Connor AM. Validation of a decisional conflict scale. Med Decis Mak Int J Soc Med Decis Mak 1995;15:25-30.

14. Fayers P, Bottomley A, EORTC Quality of Life Group, Quality of Life Unit. Quality of life research within the EORTC-the EORTC QLQ-C30. European Organisation for Research and Treatment of Cancer. Eur J Cancer 2002;38 Suppl 4:S125-133.

15. Taphoorn MJB, Claassens L, Aaronson NK, et al. An international validation study of the EORTC brain cancer module (EORTC QLQ-BN20) for assessing health-related quality of life and symptoms in brain cancer patients. Eur J Cancer 2010;46:1033-40.

16. Witlox WJA, Ramaekers BLT, Joore MA, et al. Health-related quality of life after prophy-lactic cranial irradiation for stage III non-small cell lung cancer patients: Results from the NVALT-11/DLCRG-02 phase III study. Radiother Oncol 2020;144:65-71.

17. Putora PM, Glatzer M, Belderbos J, et al. Prophylactic cranial irradiation in stage IV small cell lung cancer: Selection of patients amongst European IASLC and ESTRO experts. Radi-other Oncol 2019;133:163-6.

18. Elwyn G, Edwards A, Wensing M, et al. Shared decision making: developing the OPTION scale for measuring patient involvement. Qual Saf Health Care 2003;12:93-9.

19. Couët N, Desroches S, Robitaille H, et al. Assessments of the extent to which health-care providers involve patients in decision making: a systematic review of studies using the OPTION instrument. Health Expect 2015;18:542-61.

20. Moumjid N, Gafni A, Brémond A, et al. Shared decision making in the medical encounter: are we all talking about the same thing? Med Decis Making 2007;27:539-46.

21. Forsetlund L, Bjørndal A, Rashidian A, et al. Continuing education meetings and work-shops: effects on professional practice and health care out- 
comes. Cochrane Database Syst Rev 2009;CD003030. 22. Mokhles S, Nuyttens JJME, de Mol M, et al. Treatment selection of early stage non-small cell lung cancer: the role of the patient in clinical decision making. BMC Cancer 2018;18:79.

23. Hotta K, Kiura K, Takigawa N, et al. Desire for information and involvement in treatment decisions: lung cancer patients' preferences and their physicians' perceptions: results from Okayama Lung Cancer Study Group Trial 0705. J Thorac Oncol 2010;5:1668-72.

24. Sim JA, Shin JS, Park SM, et al. Association between information provision and decisional conflict in cancer patients. Ann Oncol 2015;26:1974-80.

25. Graham ME, Westerberg BD, Lea J, et al. Shared decision making and decisional conflict in the Management of Vestibular Schwannoma: a prospective cohort study. J Otolaryngol - Head Neck Surg 2018;47:52.

26. Chorney J, Haworth R, Graham ME, et al. Understanding shared decision making in pedi-atric otolaryngology. Otolaryngol Head Neck Surg 2015;152:941-7.

27. Rains SA. Health information seeking and the World Wide Web: an uncertainty management perspective. J Health Commun 2014;19:1296-307.

28. Ben S, Corinne F, Gijs K, et al. Prophylactic Cranial Irradiation in Extensive Small-Cell Lung Cancer. N Engl J Med 2007;664-72.

29. Takahashi T. Prophylactic cranial irradiation versus observation in patients with extensive-disease small-cell lung cancer: a multicentre, randomised, open-label, phase 3 trial. Lancet Oncol 2017;18:663-671.

30. Oronsky B, Reid TR, Oronsky A, et al. What's New in SCLC? A Review. Neoplasia 2017;19:842-7.

31. Lehman M, Gorayski P, Watson S, et al. Patient preferences regarding prophylactic cranial irradiation: A discrete choice experiment. Radiother Oncol 2016;121:225-31.

32. Putora PM, Fischer GF, Früh M, et al. Treatment of brain metastases in small cell lung cancer: Decision-making amongst a multidisciplinary panel of European experts. Radiother Oncol 2020;149:84-8. 33. Picardi C, Caparroti F, Di Maio M, et al. Prophylactic cranial irradiation in extensive disease small cell lung cancer: An endless debate. Crit Rev Oncol Hematol 2019;143:95-101. 


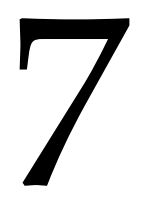

\section{Practitioners' views on shared decision-making implementation: A qualitative study}

Ankolekar, A., Steffensen, K.D., Olling, K., Dekker, A., Wee, L., Roumen, C., Hasannejadasl, H., \& Fijten, R.

PLOS One (forthcoming) 


\section{Abstract}

\section{Introduction}

Shared decision-making (SDM) refers to the collaboration between patients and their healthcare providers to make clinical decisions based on evidence and patient preferences, often supported by patient decision aids (PDAs). This study explored practitioner experiences of SDM in a context where SDM has been successfully implemented. Specifically, we focused on practitioners' perceptions of SDM as a paradigm, factors influencing implementation success, and outcomes.

\section{Methods}

We used a qualitative approach to examine the experiences and perceptions of 10 Danish practitioners at a cancer hospital experienced in SDM implementation. A semi-structured interview format was used and interviews were audio-recorded and transcribed. Data was analyzed through thematic analysis.

\section{Results}

Prior to SDM implementation, participants had a range of attitudes from skeptical to receptive. Those with more direct long-term contact with patients (such as nurses) were more positive about the need for SDM. We identified four main factors that influenced SDM implementation success: raising awareness of SDM behaviors among clinicians through concrete measurements, supporting the formation of new habits through reinforcement mechanisms, increasing the flexibility of PDA delivery, and strong leadership. According to our participants, these factors were instrumental in overcoming initial skepticism and solidifying new SDM behaviors. Improvements to the clinical process were reported. Sustaining and transferring the knowledge gained to other contexts will require adapting measurement tools.

\section{Conclusions}

Applying SDM in clinical practice represents a major shift in mindset for clinicians. Designing SDM initiatives with an understanding of 
the underlying behavioral mechanisms may increase the probability of successful and sustained implementation. 



\subsection{Introduction}

Active participation by patients in the clinical decision-making process is central to patient-centered care and can be achieved through shared decision-making (SDM) [1]. SDM has the following characteristics: (1) the clinician and patient are both involved in the decision-making process, (2) both parties exchange information with each other, (3) treatment preferences are exchanged, and (4) the final treatment decision is agreed upon by both clinician and patient [2].

These elements have been formalized by Elwyn et al. into what has come to be known as the traditional "three talk model" framework [3]. This model states that patient autonomy is built on providing highquality information and supporting the deliberation process by processing the information and arriving at an informed treatment decision. This is achieved through three steps: the choice talk, the option talk, and the decision talk. In the choice talk, the clinician makes the patient aware that there is a choice to be made between several options (in a revised version of this model this talk is referred to as a team talk to emphasize the goal of inviting the patient as a partner in the decision-making process [4].) Next, the option talk consists of the clinician providing information on these various (treatment) options. Finally, in the decision talk, the options are examined in light of the patient's preferences and a decision is made together when the patient is ready.

Recently, methods such as the SHARE approach have been developed to make the SDM process more intuitive for practitioners [5]. The SHARE approach converts SDM into a five-step process: Seek the patient's participation, Help the patient understand their options, Assess their values and preferences, Reach a decision together, and Evaluate the decision. Although the elements of the SHARE approach correspond largely with existing models such as the three-talk model, using a five-step approach is beneficial for training purposes as it assists clinicians in remembering and applying the core elements of SDM [6]. 
Yet, while these models seek to simplify the SDM process and despite positive intentions, half of clinicians do not put SDM training into practice [7]. SDM implementation is impeded by numerous barriers, both on the clinician side and the patient side. The biggest barriers cited by clinicians are (in order of frequency): time pressure, lack of applicability due to patient characteristics, lack of applicability due to the clinical situation, a perception that patients do not want to participate in decision-making, and unwillingness to ask patients about their preferred level of participation [8]. Patient-reported barriers center on logistical factors (such as a perception that clinicians have busy schedules and a perceived lack of continuity between different clinicians/departments) and consultation factors (certain patient characteristics, trust, a perceived power imbalance [9].)

Despite these barriers, a few institutes have been successful in implementing SDM in clinical practice. In Denmark, the Center for Shared Decision Making at Vejle Hospital in the region of Southern Denmark (www.cffb.dk) was one of the forerunners of a national initiative to bring policymakers, health care professionals, and patient organizations together to make healthcare more patient-centered [10]. With a focus on improving patient-centeredness in oncology, the Center successfully implemented and evaluated SDM, supported by patient decision aids (PDAs) in paper format, in the care process for several cancer patient groups, to the extent that it is now being used as a model for wider regional implementation. Evaluations of the Center's SDM initiative have shown an increase in patient engagement with no significant increase in consultation length in SDM groups compared to usual care in lung cancer and breast cancer, as well as significant reductions in decisional conflict and decisional regret for patients undergoing lung cancer diagnostics [11-13]. In addition, in a Danish national survey of patient experiences, patients rated Vejle Hospital's oncology center above the national average on most fronts, particularly clinician efforts to involve patients in decisions [14]. Till date, the perspectives of the clinicians that practice at Vejle hospital have not yet been examined and this remains a gap in the Center's existing evaluation of the 
program [15]. Understanding which aspects of the SDM implementation process clinicians find challenging and which strategies help overcome these challenges can add to our overall understanding of how to design and execute implementation initiatives that motivate clinicians to adopt these new ways of working.

The purpose of this study is to gain insight into practitioners' experiences of the introduction of a SDM initiative into their workflow at Vejle Hospital, where 'practitioners' in this context refers to individuals involved in the SDM initiative either as a clinician, nurse, team leader, or researcher. Our study was guided by three research questions centered on: (i) practitioners' initial attitudes towards SDM as a concept; (ii) their experience of the implementation process and factors that influenced implementation; and (iii) perceived outcomes. Such insights add to the current understanding of the motivational mechanisms that affect SDM implementation and may help inform similar implementation efforts in other contexts and regions.

\subsection{Methods}

\subsubsection{Study design}

We conducted a qualitative study consisting of semi-structured interviews with practitioners working at Vejle Hospital. The interview guide was created based on prior findings in the literature on barriers and facilitators to SDM and PDAs as well as prior research at MAASTRO Clinic that explored this theme [8]. In this previous MAASTRO study, oncologists had participated in semi-structured interviews to determine the barriers and facilitators for SDM and a PDA, both of which were yet to be implemented. For the present study, this interview guide was modified to account for the fact that Vejle Hospital had implemented SDM and PDAs, but the focus on barriers and facilitators remained. The interview guide (provided in the Appendix) consisted of questions along the following themes: (1) the participant's experiences with usual care and initial impressions 
of the SDM paradigm, (2) experience of SDM training, (3) using paper PDAs in practice, (4) challenges in putting SDM into practice, (5) effects of SDM on the consultation process and outcomes, (6) implementation success factors and remaining challenges.

In keeping with the semi-structured nature of the data collection, minor modifications to the interview guide were also made following the first 2-3 interviews. This was to allow participants to expand more on aspects of the implementation that they found relevant.

At the time of the study, KDS was practicing as an oncologist and researcher. $\mathrm{KO}, \mathrm{AD}, \mathrm{LW}, \mathrm{CR}$, and $\mathrm{RF}$ were researchers and $\mathrm{AA}$ and $\mathrm{HH}$ were PhD students. AA, KDS, KO, CR, and RF had prior experience in conducting qualitative research. AA, AD, LW, KDS, and RF conceived the study design and scope.

\subsubsection{Study population}

Participants were identified through KDS who was leading the Center and recruited by means of purposive sampling [16]. The main criterion was that the participant should be well-acquainted with SDM, either through direct clinical practice or general awareness by virtue of having a strategic/leadership role. In order to obtain a range of perspectives, both oncologists and oncology nurses were approached (Table 1). Ten participants agreed to be interviewed; five oncologists from four specializations, and five participants with a nursing background. The sample is reflective of the Center's strategy of applying SDM in a wide range of disease areas (aside from what are typically considered preference-sensitive conditions) and for smaller decisions along the treatment trajectory, such as nutritional decisions made with nurses. Seven participants (four oncologists and three nurses) had undergone the Center's SDM training and were applying SDM with paper PDAs in routine practice. Aside from their clinical experience, three of the participants played leadership roles in SDM implementation; two leaders were an oncologist and nurse pair based within the 
Center and responsible for implementation and evaluation efforts.

Table 7.1: Characteristics of participating clinicians

\begin{tabular}{lc}
\hline Characteristic & $\begin{array}{c}\text { No. of participants } \\
(\mathbf{N}=\mathbf{1 0})\end{array}$ \\
\hline Gender & \\
Female & 8 \\
Male & 2 \\
Experience (years) & \\
$6-10$ & 2 \\
$11-15$ & 6 \\
$16-20$ & 2 \\
Professional background & \\
Oncology & 5 \\
$\quad$ Lung cancer & 2 \\
Breast cancer & 1 \\
Gynaecological cancer & 1 \\
$\quad$ Colorectal cancer & 1 \\
Nursing & 5 \\
\hline
\end{tabular}

\subsubsection{Ethics}

According to the National Danish Consolidation Act on Research Ethics Review of Health Research Projects, Consolidation Act number 1083 of 15 September 2017 section 14 (2), notification of questionnaire surveys and medical database research projects to the system of research ethics committee system is only required if the project involves human biological material. Thus, the study was conducted without an approval from The Committee on Health Research Ethics, 
as approval for this kind of study is not required according to Danish law [17].

In accordance with general research ethics, potential participants were sent an electronic invitation with information about the purpose, aims, procedure, and the voluntary nature of the study. This information was also conveyed verbally at the start of the interviews. Participants were informed that the interview would be audio-recorded for transcription purposes and that they would be sent a written transcript of their interview with the opportunity to review it and make clarifications if desired. Participants' consent to take part was obtained prior to recording and was repeated on tape. Several measures were taken to preserve confidentiality; audio recordings were stored in a secure location and accessible to one researcher (AA) who performed all the transcription. Any personally identifying information was removed from the transcripts and each transcript was assigned a numerical identifying code.

\subsubsection{Data collection}

The interviews were conducted by AA who was a visiting researcher throughout the duration of the study and had little exposure to the clinic context beforehand, thus was in a position to analyze its SDM implementation from an external perspective. AA was not acquainted with the study participants prior to the start of the study, apart from initial acquaintance with KDS from whom she obtained approval to visit the clinic and conduct the study. Through KDS and KO, AA first made the acquaintance of potential participants in person and then followed up via email with a brief outline of the study purpose, procedure, information on participation, and an invitation to participate.

In the period between March 2020 and December 2020, seven interviews were conducted on site at the participant's clinic/office and three were conducted via video-conference due to the COVID-19 pandemic. All participants who were approached participated and 
there were no participants who dropped out of the study. Only the researcher (AA) and the participant were present in the interviews. All interviews were audio-recorded. The average duration of the interviews was 49 minutes (range: 30-71 minutes) and all interviews were transcribed verbatim from the recordings by AA.

After the interviews, participants were sent their transcript via email and given the opportunity to make changes or comments; one participant added supplementary information to their transcript. The coding process was started after the first interview and minor modifications were made to the interview guide, such as adjustments in the order, structure, and formulation of questions. Data collection continued until no new themes emerged from the participants' responses, as per the coding framework that was being constructed in parallel.

Besides the qualitative interviews, KDS arranged site meetings with SDM training developers, PDA designers, project managers, and other researchers to familiarize AA with the clinic setting. In addition, AA observed KDS's patient consultations to gain a better understanding of how SDM and PDAs are applied in practice.

\subsubsection{Data analysis}

Data was analyzed using the thematic analysis method under a reflexive approach as described by Braun and Clarke [18]. This method consists of six phases: becoming familiar with the data, generating initial codes, searching for themes, reviewing themes, generating definitions and names for each of them, and writing up the results.

The first phase consisted of reading the transcripts line by line in order to gain familiarity with the data. Next, for each transcript, initial codes were generated for fragments of text. These codes described or summarized the content of the text fragment. AA coded all the transcripts, and $\mathrm{HH}$ independently coded three transcripts. AA and $\mathrm{HH}$ then systematically compared the codes they had independently generated for each text fragment and discussed their interpretations of the text. If 
necessary, codes were rephrased or adjusted, and based on these discussions the list of codes generated across the entire dataset was built. This coding was performed manually in Microsoft Word and the coded extracts were transferred to a Microsoft Excel document in preparation for the next phase.

In phase 3, AA and $\mathrm{HH}$ grouped the codes into categories according to potential themes and sub-themes. Themes were derived from the data rather than identified in advance. These themes were reviewed in phase 4 by first re-reading the coded text fragments associated with each theme and checking whether there was coherence between the text fragments within themes, i.e. whether each theme accurately reflected the contents of the underlying text fragments. After establishing coherence within themes, the dataset was re-examined in light of the themes to check that they were representative of the data as a whole. Subsequently in phase 5, themes were refined and discussed among multiple researchers (AA, HH, RF, and CR) to ensure that they were understandable and coherent.

Analysis was conducted based on a collaborative reflexive approach in which Interpretations of the data were based on the researchers' interpretation of the data and prior theoretical knowledge. Our approach was inductive and prioritized engaging with the data and discussing multiple interpretations as opposed to achieving a specific consensus.

While it is not possible to completely eliminate interviewer bias, efforts were made to reduce confirmation bias during analysis by having another researcher $(\mathrm{HH})$ independently perform coding on a selection of interviews. This selection included interviews of clinicians, nurses, and a participant in a leadership position so that a range of perspectives were coded. The potential for leading questions and wording bias was reduced by adapting an interview guide that had been tested on multiple clinicians in a similar study conducted at MAASTRO Clinic. 


\subsection{Results}

The following section presents the themes that emerged under each of the three research questions: practitioner attitudes towards SDM, factors that influence implementation, and perceived outcomes. Our findings revealed that participants' attitudes regarding SDM prior to its implementation at Vejle Hospital ranged from skeptical to receptive. Four themes emerged under factors that influenced SDM implementation: raising awareness, leadership, reinforcement of new SDM behaviors and habits, and flexibility in PDA delivery. Strong leadership was related to the themes of raising awareness and reinforcement. Participants noted the effects of the SDM implementation on their experience of the clinical process and how measurement methods may need to be adjusted to ensure that SDM implementation is sustained. These themes and the related subthemes are summarized in the thematic map in Figure 7.1 and elaborated upon below.

\subsubsection{Attitudes towards SDM}

During the pre-implementation phase, participants recounted that although they and their colleagues were generally positive towards $\mathrm{SDM}$, there was still a degree of resistance.

\section{Initial skepticism}

Three of the clinicians recalled being initially skeptical about SDM, citing doubts about whether patients would be knowledgeable enough to actively take part in clinical decision-making, and whether the SDM steps might be too restrictive and unnatural for consultations. One participant recalled feeling that SDM might be at odds with clinicians' traditional training of ensuring progression-free survival. Another doubted whether patients would understand the consequences of their decision over the long term, and for this clinician this issue remains a concern till date. 


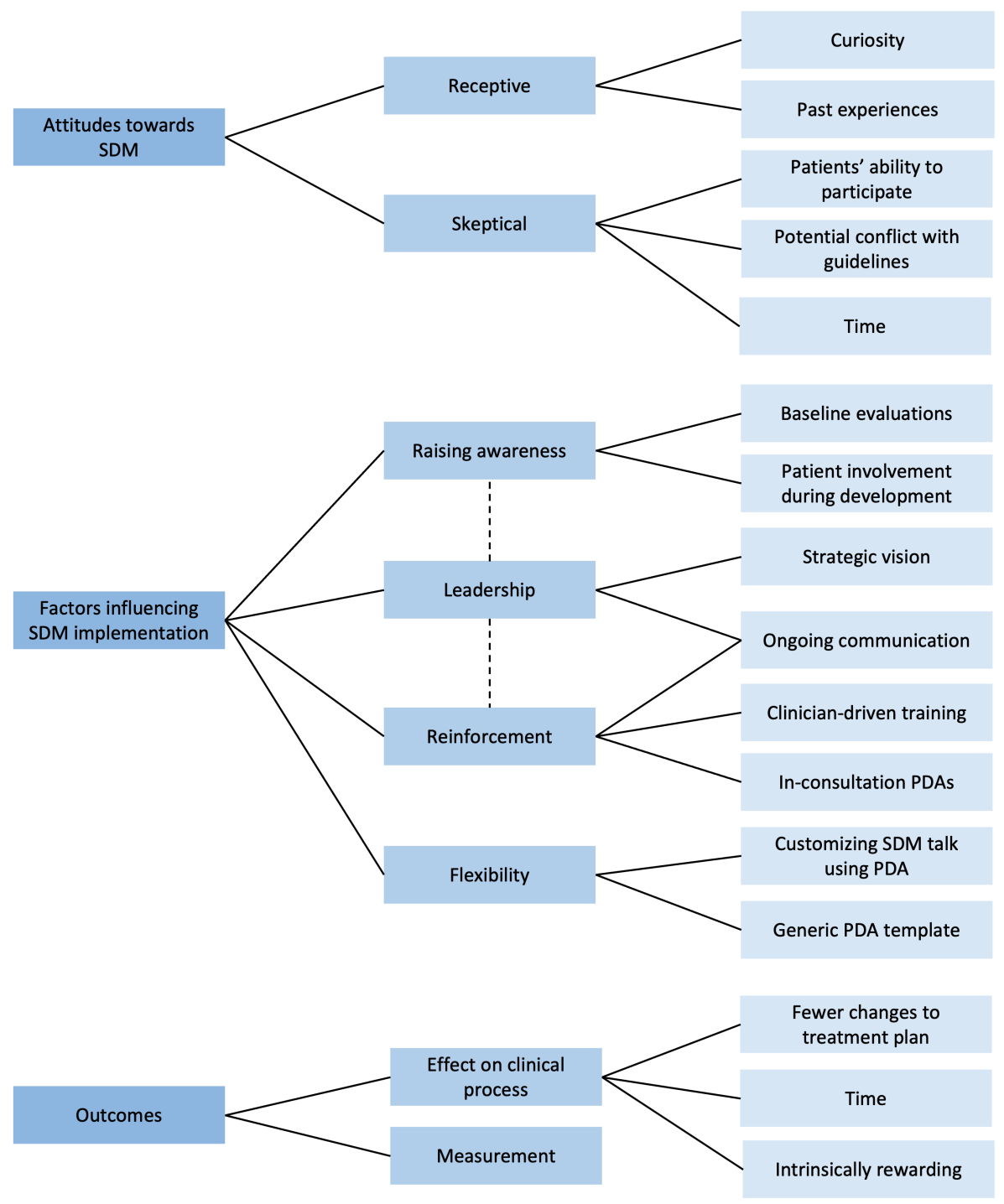

Figure 7.1: Thematic map of themes and subthemes for each research question. Solid lines indicate links between themes and subthemes, while dashed lines indicate relationships between themes. 
"I've been working with this for 15 years plus, and I give [patients] 15 minutes to make the decision. Do they actually know what is the difference between side-effects and liver metastasis?" (Participant 1)

\section{Receptive}

In contrast, four participants, primarily those with a nursing background, said that they had a positive and optimistic feeling about SDM due to their experience in seeing the long-term impact on some patients whose preferences were not incorporated in the decision. One participant described following up with an anal cancer patient who was severely affected by high dose radiotherapy and was unaware that a lower dose with less severe side-effects was an option. This participant described how hearing about SDM years later immediately triggered the thought of that patient and how much their suffering could have been avoided if the clinician had presented the option of a lower dose. For these participants, involving the patient in the decision felt like a natural and logical course of action.

"I remembered [the patient] said 'If you just had told me that I had two different options, maybe I could have chosen otherwise'. She was so sad about her situation. So, it just made sense to me to work with [SDM]." (Participant 10)

Aside from the points mentioned by clinicians interviewed, three participants who played a leadership role in the implementation also mentioned typical objections raised by clinicians in general, such as busy schedules, time constraints, and resistance to changing their way of working. They mentioned several actions that were taken to lay the groundwork for SDM implementation. These actions form the basis of the themes that emerged for part (ii) of the research question, namely practitioners' views on the factors that influenced SDM implementation. 


\subsubsection{Factors that influenced implementation}

\section{Raising awareness}

Before developing the SDM training course, the Center systematically measured the current level of patient participation and clinician efforts to involve patients by means of the OPTION-12 instrument [19]. The results were shared with clinicians, not for the purpose of evaluating individual clinicians but to give a general indication of which SDM behaviors were present in consultations and which behaviors may need to be developed. Two participants who were in a leadership role emphasized the importance of letting clinicians see SDM as an enhancement of their current practice rather than an interruption or a completely different way of working. Showing clinicians which aspects of SDM they were already performing set the stage for learning additional SDM techniques formally. For three participants, showing clinicians concrete evidence of current practice was a key step in shifting attitudes.

"You need to tell [clinicians] the good stories, you know the carrot. I think the carrot works much better than the stick." (Participant 9)

Second, involving patients alongside clinicians in PDA development helped clinicians realize that patients experience the decision-making process rather differently from what they assumed. One leader with a nursing background recalled that "including patients in that process also moves something for the clinicians. They suddenly become aware that patients are not thinking the same [way] as [clinicians] are." (Participant 10)

\section{Leadership}

Four participants emphasized the importance of strong leadership in successful implementation, beginning with the hospital's strategic vision of putting the patient first in all interactions. This led to the idea that SDM should be implemented across the trajectory, i.e. not only for major treatment decisions but also for smaller decisions made with 
nurses, such as nutrition. Therefore, the idea of SDM was brought not only to clinicians but to nurses and other support staff as well so that the patient would experience continuity and a consistent experience of being involved at all levels.

"And that has to be communicated from the top. I mean, from the leaders, that 'That is what we use here, and this is what we want to [do]. This is the way we want to talk with our patients.'" (Participant 3)

One nurse team leader also emphasized the importance of empowering clinicians to take charge of the training process and teach their peers without too much top-down management as "there's something occurring when management enters in a teaching session ... the power of the room kind of shifts a little bit. I think it's important that you keep it as a teaching room." (Participant 3)

Aside from defining the strategic vision, the involvement of leadership in practical matters was also considered important. These included keeping SDM on the agenda at staff meetings, ensuring that PDAs are being kept up-to-date and new staff members are receiving training. On-going communication between leadership and clinicians was considered particularly important. For example, several participants mentioned that it was sometimes a challenge for clinicians to come to a consensus regarding the risk information and statistical evidence the PDAs should contain, even though most patients considered this the least important aspect of the deliberation and decision-making process. In such situations, leaders played a key role in refocusing the discussion to what matters to patients.

\section{Reinforcement}

Nearly all participants described the biggest challenge in the implementation phase was for clinicians and nurses to learn new habits and adjust their way of working. Moving from the pre-implementation phase to the implementation phase involved conducting ongoing training courses and putting SDM into action 
in the clinic with the support of PDAs. This was initiated at a smaller scale at first in certain disease areas and therefore not all clinicians across the hospital had received SDM training. Seven of the participants had undergone the Center's SDM training course and described it as a one-day course added on to the mandatory two-day communication skills course for clinicians. The training was conducted by a trained clinician-nurse pair who had undergone a separate two-day SDM course based on the Train-the-trainer model [20]. The course covered the theoretical aspects of SDM, role-playing, and interactive sessions to get insights from clinicians on how to teach their peers. This course was based on the SDM model conceptualized by Elwyn et al. and was tested and refined by means of a participatory action research study that involved clinicians, patients, researchers, trainers and other stakeholders [21]. The central idea behind the training was that it should be led by clinicians themselves rather than external educators. Two participants mentioned the benefit of groups of clinicians learning SDM together and from each other as a shared experience that helped reinforce the teachings.

"It took some effort, but the other doctors and the nurses around me [did] the same course, so we had the same language about it. And in my experience, it was like re-visualizing my conversation. So it's more fun being a doctor this way." (Participant 7)

The tangible paper PDAs were regarded as a key element in solidifying the knowledge gained in the training course and putting it into practice. One leader recalled that making sure the PDAs would actually be used in the consultation was a key consideration in their design and format. The risk of "over-the-counter" PDAs that are given to patients for use at home is that clinicians may consider it a quick fix and assume that the patient has been informed about their options sufficiently through the PDA. This may reduce the incentive to formally go through the SDM process and make it easier to relapse into traditional decision-making. For this reason, the Center opted to make the PDAs physical in-consultation tools. Four participants described how the PDA served not only as a supporting tool for the SDM process 
but as a learning tool for clinicians; having a tangible PDA provided a structure for the SDM conversation and a way to move from one step to another.

"Sometimes I think that [the PDA] is mostly to help the doctors. ... And for me, it's also easier in my mind to structure the conversation. And then the clue is to use the [PDA] from the beginning." (Participant 7)

\section{Flexibility}

The paper PDAs were designed according to the traditional three-talk SDM model [3]. Each PDA was in the form of a folder with five components corresponding to the five steps of the SDM model, and contained multiple cards describing the benefits and harms of each treatment option, patient stories, and statistics about survival and side-effects. The cards could then be spread out on the table to provide both patient and clinician with a shared visual overview of the information and treatment options. Some participants reported that this gave them the flexibility to tailor the SDM conversation to the factors that mattered to the patient, for instance by moving the cards around in order of preference or removing cards that the patient felt were not relevant to them.

Three participants mentioned the challenges in translating knowledge about SDM from one context to another, including building new PDAs for different disease areas and keeping them up-to-date. At Vejle Hospital, this challenge was addressed by placing a generic version of the paper PDA on a web-based platform for clinicians and nurses to edit. This gave them the flexibility to update existing PDAs based on new clinical evidence or create new PDAs for different decision-making contexts. 


\subsubsection{Outcomes}

When participants reflected on the outcomes of the SDM initiative on their practice, two themes emerged: improvements in the clinical process and measurement.

\section{Effects on the clinical process}

Participants considered the prime benefit of SDM to be that the consultation was more focused towards what is relevant for patients. Learning about the patient's lifestyle, activities, and future plans, among other things, allowed the clinician to frame information in terms of impact on the patient's daily life and avoid overloading the patient with clinical jargon. One participant described the process of engaging with each patient as an individual intrinsically rewarding, while another felt that sharing the process with the patient makes the clinician's job easier due to the confidence that the right decision is being made for the patient. Two participants observed better treatment compliance and fewer changes to the treatment plan.

"My [anecdotal] experience is that by performing SDM whenever you have a new patient, the [treatment] plan you make is more often being completed. I used to adapt plans much more often earlier because they didn't tolerate the treatment or they were not confident that it was the right thing. They needed extra consultations." (Participant 7)

When asked about the effects of SDM on consultation length, participants said that SDM consultations were not significantly longer than usual care in their experience. According to two participants, SDM might take some extra minutes but they emphasized that it is worth the time investment to make a better decision. In addition, choosing the right treatment from the start may mean fewer consultations needed in the future. A third benefit was that patients feel more confident about treatment decisions and exhibit better compliance, lending support to the idea that a lengthier consultation may not be a barrier to SDM if there is a visible improvement in clinical outcomes. 
"It maybe takes a few minutes more, but then the thing is, isn't that okay? Isn't it okay to spend a few minutes? You're going to take this treatment, maybe chemotherapy for 4-6 months. You're going to have radiotherapy, endocrine treatment for five or 10 years, I think two minutes [more] - that's okay ... that we spend a little bit of time if we have better compliance, if the patient feels they're more involved." (Participant 1)

\section{Measurement}

Experiencing SDM in practice also led some participants to reflect on changes that may be needed at the organizational level for sustained implementation. One participant noted that current performance metrics are based on activity, such as the number of consultations performed, and do not account for the fact that patient choices may deviate from standard procedure under SDM. This participant recognized the need for value-based rather than activity-based metrics and emphasized the importance of cooperation and communication between department managers/supervisors and clinicians.

Transferring the knowledge gained from local implementation to the regional level and ensuring that implementation stays on track over the long-term was seen as another set of challenges. Current methods to evaluate SDM focus on the quality of the clinical interaction and outcomes. Some participants felt that an additional multidimensional measure of SDM implementation may be needed in order to evaluate the progress at the department level. Possible dimensions mentioned were the level of clinician skills, organizational/cultural readiness, and leadership. The emphasis was on understanding local contexts and adapting the Center's knowledge and experience accordingly.

"We always have to adapt to the culture. So we can't just, you know, do a complete package and say, 'Here you go, you should do this.' We have to adjust all the time." (Participant 10) 


\subsection{Discussion}

Although healthcare is becoming increasingly patient-centered, SDM is yet to become the norm in most countries [22]. Even when clinicians are enthusiastic about patient engagement, misperceptions about what constitutes SDM, what distinguishes it from informed decisionmaking, and a lack of organizational support can thwart implementation efforts [23]. As a result, SDM and PDA uptake may fail to be sustained over time [24]. Within this context, we studied SDM implementation at Vejle Hospital, Denmark, where, under the leadership of the Center for Shared Decision Making, SDM and PDAs have been implemented with considerable success [11-13]. The purpose of this qualitative study was to examine practitioners' experiences of the Center's efforts to introduce SDM and PDAs into clinical practice. In doing so, we provide insights into the factors that may play a role in the hospital's sustained success in implementing SDM. Our analysis revealed challenges to SDM spanning the pre-implementation to post-implementation phase and the strategies that were used to address them. These include overcoming clinician resistance, ensuring that training and tools are successfully put into practice, and facilitating sustained implementation efforts over the long-term through appropriate performance metrics and continuous communication between the various stakeholders (clinicians, nurses, team leaders, and researchers).

Frequently cited objections to SDM include doubt about its applicability to the clinician situation or the individual patient and perceptions that it will disrupt busy workflows $[25,26]$. These perceptions were present in the pre-implementation phase in our study and can result in resistance towards adopting a different way of conducting consultations [27]. There was a pronounced difference in our results in how clinicians and nurses viewed patient participation; nurses were enthusiastic about SDM due to their first-hand experience seeing the consequences of not taking patients' wishes into account in treatment decisions, whereas for clinicians this shift happened after working with patients on developing the PDAs and was reinforced through repeated 
application of SDM principles. Previous findings from the Center have shown that pre-implementation OPTION scores indicated lower levels of SDM [11]. Communicating this disparity to clinicians may be the first step in shifting attitudes, as clinicians' perception that SDM improves their practice makes sustained implementation more likely [28].

Interestingly, while time constraints are the most frequently cited barrier to SDM in the literature, this objection was not present in our results. A previous study conducted at the Center which assessed the impact of SDM and PDAs on consultation length found no significant increase, and in fact a decrease in variability of consultation lengths [12]. The authors speculate that the structured nature of the SDM consultations owing to the PDA format makes consultations more standardized in terms of procedure. The results of similar studies using in-consultation PDAs suggest that this format may be ideal for clinicians concerned about time constraints $[29,30]$.

Another factor in increasing clinician motivation was empowering clinicians to take the lead in learning and training each other in SDM, particularly with minimal direct involvement from management. The Center has previously described its approach to creating ownership through involvement and this approach extended to its SDM training course, which is based on the Train-the-trainer model in which clinicians become trained in training their colleagues, thus becoming ambassadors for SDM $[15,20]$. This approach has been associated with positive results in various contexts, with the importance of a clinical ambassador or champion being emphasized in multiple studies [31-34]. Currently, there is no consensus in the literature regarding which training approach works best for teaching SDM in a clinical setting; measuring training effectiveness is a complex endeavor because there is no generally accepted best measure of SDM [35]. Training evaluation metrics tend to focus on the level of knowledge clinicians gained and indirect outcome measurements such as impact of the training on patient satisfaction and quality of the clinical interaction, while neglecting clinicians' evaluations and satisfaction of 
their training [36]. While outcome measures are important metrics, it is also necessary to assess clinician satisfaction with the training itself, as positive appraisal has a significant impact on the motivation to learn from the training and put its principles into practice [37].

Moving from training to regular practice also requires measures to facilitate the development of new habits, provision of appropriate resources, and social support [38]. Previous findings revealed that even in spite of positive intentions, only half of clinicians put SDM training into practice [7]. Our findings suggest that the form and function of PDAs played a key role in this regard. A number of our participants considered the PDA to be as much a supporting tool for clinicians as it was for patients. Using a tangible PDA that is structured according to the steps of the SDM model can help reinforce the SDM training through repetition. Evidence from the Center shows that between two groups of clinicians who had received SDM training, the level of patient engagement and SDM measured by OPTION scores was significantly higher when a PDA was used [11]. This suggests that while training on its own may improve clinicians' knowledge and skills to an extent, the repetition of using the five-step PDA may help solidify the contents of the training till it becomes routine [39]. There is preliminary evidence of PDAs being used as 'scaffolding' to facilitate SDM consultations, and certain PDA formats such as decision cards are specifically designed to function as collaborative tools rather than purely information tools $[40,41]$. However, we are unable to draw firm conclusions from this result as the overwhelming majority of PDAs are designed for patients to use outside the consultation [42].

Although PDAs are increasingly being offered in a digital format, preliminary evidence suggests that, given a choice, patients tend to use paper PDAs more frequently than digital PDAs and rate them higher in terms of overall satisfaction $[43,44]$. While both formats increase patient knowledge and users of digital PDAs tend to perform the values clarification function at a higher rate, the limited evidence still points to a digital divide in which there is a preference for print-based PDAs. Practitioners aiming to use PDAs as a supporting tool for SDM would 
therefore benefit from investigating their patients' needs and preferences regarding decision support format as well as considering what role PDAs would play in the clinical workflow, i.e. whether they are deployed purely as an informational tool outside the consultation or used within the consultation to guide the SDM process.

It is also important to note that tools such as PDAs, while useful as supporting material, cannot compensate for the relational aspect of the SDM process. The core of SDM is effective communication between patients and clinicians [45]. Furthermore, it may not be possible to have a PDA for each clinical situation, and events such as the COVID-19 pandemic, in which some consultations must be conducted remotely, further underscore the need for empathic patient-centered communication [46,47]. This extends beyond the patient-clinician interaction to the way patients interact with the healthcare system as a whole. Steps taken to build patients' trust ensure that SDM initiatives do not operate in isolation at the point of decision-making but become part of a culture of care that prioritizes the patient at all points of the pathway [48]. According to our participants, Vejle hospital's 'Patient First' vision was the impetus for its SDM initiative, suggesting that SDM was a means to an end rather than an end in itself. This distinction has been emphasized by several authors, and empirical evidence highlights the importance of a shared vision, i.e. a specific 'end', in effective implementation [49-51]. This theme has not been extensively explored in the SDM context and could be a valuable area for future research.

The importance of leadership in sustaining implementation efforts over time was emphasized by multiple participants in our study. Leadership that is unsupportive of change can be a barrier to implementation, and leaders play a key role in shaping the environments that favor sustained application of training and PDAs over the long-term [52]. Alongside the regular SDM training for clinicians, the Center also developed a 3-hour training program geared towards leaders to acquaint them with the principles of SDM to help foster a positive implementation environment, according to $\mathrm{K}$. 
Dahl Steffensen, MD, PhD (written communication, June 2021). Such initiatives can support the creation of a "learning health care system" in which performance metrics are aligned with the organizational vision for SDM implementation [53]. For instance, involving patients through SDM can shift previously 'traditional' treatment patterns based on clinical guidelines to more individualized patterns based on patient preferences; some patients may prefer less intensive treatments, some may choose to forgo certain treatments altogether, or otherwise deviate from guidelines [54]. Health care systems must account for this. Clinical departments that are evaluated using activity-based metrics, such as number of procedures performed or the number of consultations conducted, may have misaligned incentives that hinder SDM implementation [55]. This issue is increasingly on the policy agenda in several countries around the world, including Denmark, although the evidence of current value-based models is limited [56]. While hospital managers and team leaders must be aware of such models and their implications within their own contexts, wider actions at the policy level are needed to put such changes into motion.

Strengths and limitations This study was conducted in a context in which SDM has been relatively successfully implemented and it is therefore possible that participants had mostly positive attitudes towards SDM and the type of PDAs used owing to their experiences. As such, our study may be affected by selection bias. We have attempted to get a range of perspectives by interviewing participants with varying degrees of exposure to SDM principles, however the small-scale nature of this study allows us to draw tentative conclusions on how best to support SDM initiatives among clinicians.

Qualitative research is often said to lack generalizability, and another limitation of our findings could be the extent to which they hold up in other contexts and countries. Cultures vary even within different hospitals and departments, and organizational culture is considered to be the most crucial factor that influences implementation success [57]. There is a lack of studies exploring specific strategies to change 
organizational culture in order to improve health care processes, but the critical role of leadership has been well-established [58,59]. Culture can emerge by default or be built purposefully, and it is important for leaders at all organizational levels (from hospital administrators to team leaders) to understand their role in shaping the culture [60,61]. Furthermore, the success factors we identified (training, tools, and leadership) do not operate in isolation, and so an effective implementation strategy must consider the interplay between the various factors identified [57].

Another limitation of our study is that we focus exclusively on the clinician perspective of SDM implementation and we do not include the patient perspective which is central to SDM. As a result, while our findings give insight into factors that may help clinicians practice SDM, we are not able to comment on how patients experience the process. Further research is needed as patients do not always feel involved or experience SDM despite clinicians' best efforts [62,63]. Although patients were heavily involved during the development and design phase of the Center's SDM initiative, further qualitative research into patients' experiences of the SDM process may provide a more comprehensive picture of successful implementation. Possible research questions may include how involved patients felt in their treatment decision under the SDM process, their experiences with using the paper PDAs, and the impact of their choices on their quality of life.

\subsection{Conclusion}

This study describes practitioners' insights and experiences regarding SDM implementation in their practice. We found that prior to implementation, many clinicians harbored a degree of skepticism about the value and practicality of SDM. Under factors that facilitated SDM implementation, four main themes emerged: raising awareness, leadership, reinforcement, and flexibility. Systematically measuring the level of patient involvement prior to SDM implementation helped make clinicians aware of the gap between current practice and an ideal 
Chapter 7. Practitioners' views on shared decision-making implementation

SDM process. Supporting clinicians by means of role-play training and in-consultation PDAs helped in reinforcing the new habits associated with SDM so that implementation could be sustained. Sustainability was reinforced by creating an online platform where generic PDA templates could be customized by clinicians and/or nurses, allowing for greater flexibility and adaptability to different clinical contexts. Strong leadership was instrumental in driving these changes.

In conclusion, putting SDM principles into practice successfully requires clinicians to learn new behaviors and actions. This process may be facilitated by combining a top-down and bottom-up approach in which clinician leaders guide SDM initiatives while empowering clinicians to create supporting tools and learn from each other. 


\section{Appendix}

Semi-structured interview guide

1. Could you tell me a little bit about your background and experiences in the clinic?

2. How would you characterize your experiences with patients under traditional decision-making, i.e. before the Center's shared decision-making (SDM) initiative?

3. When you encountered the idea of SDM, what was your initial reaction or impression?

4. In your experience, how do patients tend to react when they are invited to take part in SDM consultations?

5. Could you tell me about the Center's development process for SDM and patient decision aids (PDAs)?

a) Who were the stakeholders who participated in designing the PDAs and SDM training?

b) What factors did these stakeholders consider important when designing the PDAs and SDM training?

6. How easy or difficult was it in the beginning to apply your knowledge of SDM from training into practice in the actual consultation? What would you say were the biggest challenges and what factors, if any, did you find helpful?

7. Could you tell me about your experiences with using paper PDAs to conduct SDM consultations?

a) Besides the clinician, who participates in the SDM consultation with the patient and how are their roles defined? (E.g. nurses)

b) How are PDAs introduced and used during the SDM consultation? 
Chapter 7. Practitioners' views on shared decision-making implementation

c) How are the PDAs kept up-to-date and typically who is responsible for maintaining the PDAs?

d) How do you explain the various treatment risks and sideeffects to the patient?

e) In your experience, how long does a typical SDM consultation last? Do you feel that your SDM consultations take additional time?

8. What was your experience of the effects of SDM on the consultation process and on outcomes?

9. Do you still experience/observe challenges in carrying out the SDM consultations or using the PDAs? What factors do you think would be helpful to you in reducing these challenges? 


\section{References}

1. Institute of Medicine (US) Committee on Quality of Health Care in America. Crossing the Quality Chasm: A New Health System for the 21st Century [Internet]. Washington (DC): National Academies Press (US); 2001 [cited 2021 Jan 11]. Available from: http://www.ncbi.nlm.nih.gov/books/NBK222274/

2. Charles C, Gafni A, Whelan T. Decision-making in the physician-patient encounter: revisiting the shared treatment decision-making model. Soc. Sci. Med. 1982 1999;49:651-61.

3. Elwyn G, Frosch D, Thomson R, Joseph-Williams N, Lloyd A, Kinnersley $\mathrm{P}$, et al. Shared decision making: a model for clinical practice. J. Gen. Intern. Med. 2012;27:1361-7.

4. Elwyn G, Durand MA, Song J, Aarts J, Barr PJ, Berger Z, et al. A threetalk model for shared decision making: multistage consultation process. BMJ 2017;j4891.

5. Agency for Healthcare Research and Quality. The SHARE Approach-Using the Teach-Back Technique: A Reference Guide for Health Care Providers. 2014;

6. Sturgess J, Clapp JT, Fleisher LA. Shared decision-making in peri-operative medicine: a narrative review. Anaesthesia 2019;74:13-9.

7. Boland L, Lawson ML, Graham ID, Légaré F, Dorrance K, Shephard A, et al. Post-training Shared Decision Making Barriers and Facilitators for Pediatric Healthcare Providers: A Mixed-Methods Study. Acad. Pediatr. 2019;19:118-29.

8. Gravel K, Légaré F, Graham ID. Barriers and facilitators to implementing shared decision-making in clinical practice: a systematic review of health professionals' perceptions. Implement. Sci. 2006;1:16.

9. Joseph-Williams N, Elwyn G, Edwards A. Knowledge is not power for patients: A systematic review and thematic synthesis of patient-reported barriers and facilitators to shared decision making. Patient Educ. Couns. 2014;94:291-309.

10. Dahl Steffensen K, Hjelholt Baker V, Vinter MM. Implementing shared decision making in Denmark: First steps and future focus areas. Z. Für Evidenz 
Fortbild. Qual. Im Gesundheitswesen 2017;123-124:36-40.

11. Olling K, Stie M, Winther B, Steffensen KD. The impact of a patient decision aid on shared decision-making behaviour in oncology care and pulmonary medicine-A field study based on real-life observations. J. Eval. Clin. Pract. 2019;25:1121-30.

12. Søndergaard SR, Madsen PH, Hilberg O, Bechmann T, Jakobsen E, Jensen $\mathrm{KM}$, et al. The impact of shared decision making on time consumption and clinical decisions. A prospective cohort study. Patient Educ. Couns. 2021;104:1560-7.

13. Søndergaard SR, Madsen PH, Hilberg O, Jensen KM, Olling K, Steffensen $\mathrm{KD}$. A prospective cohort study of shared decision making in lung cancer diagnostics: Impact of using a patient decision aid. Patient Educ. Couns. 2019;102:1961-8.

14. Center for Patient Involvement. Center for Patient Experience and Evaluation: Danish National Survey on Patient Experiences LUP, 2017. https://patientoplevelser.dk/files/dokumenter/filer/LUP/ LUP2016/lup_2016_rapport.pdf.

15. Steffensen KD, Vinter M, Crüger D, Dankl K, Coulter A, Stuart B, et al. Lessons in Integrating Shared Decision-Making Into Cancer Care. J. Oncol. Pract. 2018;14:229-35.

16. Palinkas LA, Horwitz SM, Green CA, Wisdom JP, Duan N, Hoagwood K. Purposeful sampling for qualitative data collection and analysis in mixed method implementation research. Adm. Policy Ment. Health Ment. Health Serv. Res. 2015;42:533-44.

17. Act on Research Ethics Review of Health Research Projects [Internet]. 2018;Available from: https://en.nvk.dk/rules-and-guidelines/act-onresearch-ethics-review-of-health-research-projects

18. Braun V, Clarke V. Using thematic analysis in psychology. Qual. Res. Psychol. 2006;3:77-101.

19. Elwyn G, Hutchings H, Edwards A, Rapport F, Wensing M, Cheung W, et al. The OPTION scale: measuring the extent that clinicians involve patients in decision-making tasks. Health Expect. 2005;8:34-42.

20. Orfaly RA, Frances JC, Campbell P, Whittemore B, Joly B, Koh H. Train- 
the-trainer as an Educational Model in Public Health Preparedness. :5.

21. Ammentorp J, Wolderslund M, Timmermann C, Larsen H, Steffensen $\mathrm{KD}$, Nielsen A, et al. How participatory action research changed our view of the challenges of shared decision-making training. Patient Educ. Couns. 2018;101:639-46.

22. Couët N, Desroches S, Robitaille H, Vaillancourt H, Leblanc A, Turcotte $\mathrm{S}$, et al. Assessments of the extent to which health-care providers involve patients in decision making: a systematic review of studies using the OPTION instrument. Health Expect. 2015;18:542-61.

23. Moumjid N, Gafni A, Brémond A, Carrère M-O. Shared decision making in the medical encounter: are we all talking about the same thing? Med. Decis. Mak. Int. J. Soc. Med. Decis. Mak. 2007;27:539-46.

24. Pollard S, Bansback N, Bryan S. Physician attitudes toward shared decision making: A systematic review. Patient Educ. Couns. 2015;98:1046-57.

25. Légaré F, Ratté S, Gravel K, Graham ID. Barriers and facilitators to implementing shared decision-making in clinical practice: update of a systematic review of health professionals' perceptions. Patient Educ. Couns. 2008;73:526-35.

26. Légaré F, Thompson-Leduc P. Twelve myths about shared decision making. Patient Educ. Couns. 2014;96:281-6.

27. Munro S, Manski R, Donnelly KZ, Agusti D, Stevens G, Banach M, et al. Investigation of factors influencing the implementation of two shared decision-making interventions in contraceptive care: a qualitative interview study among clinical and administrative staff. Implement. Sci. 2019;14:95.

28. Coylewright M, O'Neill E, Sherman A, Gerling M, Adam K, Xu K, et al. The Learning Curve for Shared Decision-making in Symptomatic Aortic Stenosis. JAMA Cardiol. 2020;5:442.

29. LeBlanc A, Wang AT, Wyatt K, Branda ME, Shah ND, Van Houten H, et al. Encounter Decision Aid vs. Clinical Decision Support or Usual Care to Support Patient-Centered Treatment Decisions in Osteoporosis: The Osteoporosis Choice Randomized Trial II. PLOS ONE 2015;10:e0128063.

30. Whelan T, Sawka C, Levine M, Gafni A, Reyno L, Willan A, et al. Helping patients make informed choices: a randomized trial of a decision aid for ad- 
juvant chemotherapy in lymph node-negative breast cancer. J. Natl. Cancer Inst. 2003;95:581-7.

31. Kienlin S, Poitras M-E, Stacey D, Nytrøen K, Kasper J. Ready for SDM: evaluating a train-the-trainer program to facilitate implementation of SDM training in Norway. BMC Med. Inform. Decis. Mak. 2021;21:140.

32. Lee SWH, Mak VSL. Train-the-trainer program on cardiovascular health for community pharmacists in Malaysia. Int. J. Clin. Pharm. 2017;39:1166-70.

33. Sonntag U, Koch A, Bayer G, Heintze C, Döpfmer S. Train the trainer course for general practice trainers in ambulatory care: the Berlin model. GMS J. Med. Educ. 2020;37:Doc28.

34. Scholl I, LaRussa A, Hahlweg P, Kobrin S, Elwyn G. Organizational- and system-level characteristics that influence implementation of shared decisionmaking and strategies to address them - a scoping review. Implement. Sci. 2018;13:40.

35. Gärtner FR, Bomhof-Roordink H, Smith IP, Scholl I, Stiggelbout AM, Pieterse AH. The quality of instruments to assess the process of shared decision making: A systematic review. PloS One 2018;13:e0191747.

36. Müller E, Strukava A, Scholl I, Härter M, Diouf NT, Légaré F, et al. Strategies to evaluate healthcare provider trainings in shared decision-making (SDM): a systematic review of evaluation studies. BMJ Open 2019;9:e026488.

37. Kirkpatrick D, Kirkpatrick J. Evaluating training programs: The four levels. Berrett-Koehler Publishers; 2006.

38. Kwasnicka D, Dombrowski SU, White M, Sniehotta F. Theoretical explanations for maintenance of behaviour change: a systematic review of behaviour theories. Health Psychol. Rev. 2016;10:277-96.

39. Agbadjé TT, Elidor H, Perin MS, Adekpedjou R, Légaré F. Towards a taxonomy of behavior change techniques for promoting shared decision making. Implement. Sci. 2020;15:67.

40. Elwyn G, Edwards A, Hood K, Robling M, Atwell C, Russell I, et al. Achieving involvement: process outcomes from a cluster randomized trial 
of shared decision making skill development and use of risk communication aids in general practice. Fam. Pract. 2004;21:337-46.

41. Montori VM, Breslin M, Maleska M, Weymiller AJ. Creating a Conversation: Insights from the Development of a Decision Aid. PLoS Med. 2007;4:e233.

42. Elwyn G, Frosch D, Volandes AE, Edwards A, Montori VM. Investing in Deliberation: A Definition and Classification of Decision Support Interventions for People Facing Difficult Health Decisions. Med. Decis. Making 2010;30:701-11.

43. Stacey D, Suwalska V, Boland L, Lewis KB, Presseau J, Thomson R. Are Patient Decision Aids Used in Clinical Practice after Rigorous Evaluation? A Survey of Trial Authors. Med. Decis. Making 2019;39:805-15.

44. Tomko C, Davis KM, Luta G, Krist AH, Woolf SH, Taylor KL. A Comparison of Web-Based Versus Print-Based Decision Aids for Prostate Cancer Screening: Participants' Evaluation and Utilization. J. Gen. Intern. Med. 2015;30:33-42.

45. Hargraves I, LeBlanc A, Shah ND, Montori VM. Shared Decision Making: The Need For Patient-Clinician Conversation, Not Just Information. Health Aff. (Millwood) 2016;35:627-9.

46. Abrams EM, Shaker M, Oppenheimer J, Davis RS, Bukstein DA, Greenhawt M. The Challenges and Opportunities for Shared Decision Making Highlighted by COVID-19. J. Allergy Clin. Immunol. Pract. 2020;8:2474-2480.e1.

47. Marron JM, Joffe S, Jagsi R, Spence RA, Hlubocky FJ. Ethics and Resource Scarcity: ASCO Recommendations for the Oncology Community During the COVID-19 Pandemic. J. Clin. Oncol. 2020;38:2201-5.

48. Berry LL, Awdish RLA, Letchuman S, Steffensen KD. Trust-Based Partnerships Are Essential - and Achievable - in Health Care Service. Mayo Clin. Proc. 2021;S0025619621002652.

49. Clarke A, Paraskeva N, White P, Tollow P, Hansen E, Harcourt D. PEGASUS: the Design of an Intervention to Facilitate Shared Decision-making in Breast Reconstruction. J. Cancer Educ. 2021;36:508-18.

50. Barry MJ, Edgman-Levitan S, Sepucha K. Shared Decision-Making: stay- 
ing focused on the ultimate goal. NEJM Catal. 2018;4.

51. Pearce CL, Ensley MD. A reciprocal and longitudinal investigation of the innovation process: the central role of shared vision in product and process innovation teams(PPITs). J. Organ. Behav. 2004;25:259-78.

52. Joseph-Williams N, Abhyankar P, Boland L, Bravo P, Brenner AT, Brodney $\mathrm{S}$, et al. What Works in Implementing Patient Decision Aids in Routine Clinical Settings? A Rapid Realist Review and Update from the International Patient Decision Aid Standards Collaboration. Med. Decis. Making 2020;0272989X2097820.

53. Friedman C, Rubin J, Brown J, Buntin M, Corn M, Etheredge L, et al. Toward a science of learning systems: a research agenda for the high-functioning Learning Health System. J. Am. Med. Inform. Assoc. JAMIA 2015;22:43-50.

54. Stacey D, Légaré F, Lewis K, Barry MJ, Bennett CL, Eden KB, et al. Decision aids for people facing health treatment or screening decisions. Cochrane Database Syst. Rev. [Internet] 2017 [cited 2020 Dec 15];Available from: http:/ /doi.wiley.com/10.1002/14651858.CD001431.pub5

55. Burau V, Dahl HM, Jensen LG, Lou S. Beyond Activity Based Funding. An experiment in Denmark. Health Policy 2018;122:714-21.

56. Milstein R, Schreyoegg J. Pay for performance in the inpatient sector: A review of $34 \mathrm{P} 4 \mathrm{P}$ programs in 14 OECD countries. Health Policy Amst. Neth. 2016;120:1125-40.

57. Li S-A, Jeffs L, Barwick M, Stevens B. Organizational contextual features that influence the implementation of evidence-based practices across healthcare settings: a systematic integrative review. Syst. Rev. 2018;7:72.

58. Parmelli E, Flodgren G, Beyer F, Baillie N, Schaafsma ME, Eccles MP. The effectiveness of strategies to change organisational culture to improve healthcare performance: a systematic review. Implement. Sci. IS 2011;6:33.

59. Warrick DD. What leaders need to know about organizational culture. Bus. Horiz. 2017;60:395-404.

60. Azzolini E, Ricciardi W, Gray M. Healthcare organizational performance: why changing the culture really matters. Commentary. Ann. Ist. Super. Sanita 2018;54:6-8. 
61. Damschroder LJ, Aron DC, Keith RE, Kirsh SR, Alexander JA, Lowery JC. Fostering implementation of health services research findings into practice: a consolidated framework for advancing implementation science. Implement. Sci. 2009;4:50.

62. Joseph-Williams N, Lloyd A, Edwards A, Stobbart L, Tomson D, Macphail $\mathrm{S}$, et al. Implementing shared decision making in the NHS: lessons from the MAGIC programme. BMJ 2017;j1744.

63. Finderup J, Dam Jensen J, Lomborg K. Evaluation of a shared decisionmaking intervention for dialysis choice at four Danish hospitals: a qualitative study of patient perspective. BMJ Open 2019;9:e029090. 



\section{General discussion}




\subsection{Introduction}

The nature of clinical decision-making is changing, particularly in oncology. The increasing number of treatment modalities, the potential for personalized medicine due to advances in genomics, and the prominence of patient autonomy cause patients to become partners in the decision-making process rather than bystanders. Initiatives such as shared decision-making (SDM) aim to facilitate this increased patient involvement. Decision support tools such as patient decision aids (PDAs) and clinical decision support systems (CDSSs) are being developed to provide patients and clinicians with the necessary information required to make a good clinical decision. However, despite the increasing prominence of SDM in medical literature and on several national agendas around the world, many implementation efforts falter. Creating and integrating SDM and related organizational changes into routine care is challenging as it requires change on multiple levels, from the individual and behavioral to the organizational. A deeper understanding of these various facets is needed to ensure that SDM does not remain a buzzword.

In this doctoral thesis we explored the methods, challenges, and opportunities for supporting cancer patients to actively participate in their treatment decisions through SDM. We conducted three studies on SDM implementation: the first study involved developing and implementing a PDA in prostate cancer (Chapters 3 and 4), the second was an investigation of treatment decisions in lung cancer and the possibility for SDM using CDSSs (Chapters 5 and 6), and the final study explored the characteristics of a successful SDM implementation at a Danish cancer hospital (Chapter 7). These studies offered a variety of contexts in which to explore the original research questions of this thesis:

1. How to develop a PDA that fulfills user requirements and clinical standards?

2. What do clinicians perceive as barriers and facilitators to implementing SDM in prostate cancer/preference-sensitive condi- 
tions?

3. How do these barriers and facilitators manifest in late-stage cancers, for example lung cancer, that are less preference-sensitive?

4. How can SDM and PDA delivery be optimized to account for the barriers and facilitators?

We will begin by summarizing the main findings from our studies in light of these research questions. We will then consider the strengths and limitations of our research and discuss the implications of our findings. We will conclude with implications for practitioners and for future research.

\subsection{Summary of main findings}

\subsubsection{Developing an acceptable PDA (research question 1)}

In Chapter 2, we reviewed various aspects of patient decision aids (PDAs) in general: what they are, how they are developed, what opportunities they offer as a part of the SDM process, and their limitations. Reviewing the most prominent studies including systematic reviews, we showed that developing a PDA that is acceptable according to international and clinical standards such as the International Patient Decision Aid Standards (IPDAS) [1]. However, this is a multistage process because many factors need to be taken into consideration, the foremost being that the clinical information contained in the PDA must be accurate according to clinicians, and understandable to patients. Second, the format the information is provided in must be user-friendly, particularly for patients who may not be accustomed to using digital decision support. Finally, the PDA must be embedded into the clinical workflow. This requires that clinicians must be familiar with its contents, and there must be adequate time in the consultation to discuss its outputs. This may also involve addressing technical challenges such as incorporating the PDA into the hospital's electronic health record (EHR) system to ensure that clinicians and patients both 
have access to the latest information filled in by the patient.

In Chapter 3, we designed a web-based PDA for prostate cancer patients by means of a user-centered design process. Former prostate cancer patients, clinicians, usability experts, and volunteers gave their inputs and feedback on successive prototypes of the PDA until consensus was reached. Two main conclusions were drawn from this study. The first was that patients and clinicians evaluate treatment options differently, albeit with some overlap. Clinicians tend to focus on providing enough clinical information to patients so that they can make decisions based on complete information. While patients found these clinical details important, many also focused on other aspects of the treatment trajectory, such as the number of hospital visits required, the surgical procedure, and the longer-term impact of different treatments on the patient's quality of life were important considerations for former prostate cancer patients. These considerations needed to be added to the PDA so that patients could reflect on what mattered to them.

The second conclusion was a concern that introducing a PDA and discussing its output with patients in an SDM process would add time to clinicians' busy workflows. The output in this context was the level of patient knowledge as determined by a short quiz in the PDA and the relative importance of different treatment aspects as rated by the patient in the values clarification component. Although the evidence is not conclusive that SDM lengthens consultations, the fact that this is a concern for many clinicians needs to be taken into account when introducing a PDA into the clinical workflow. We attempted to address this concern by providing the PDA's output in a simple tabular format that could provide a quick overview of the patient's main concerns and provide some structure to the consultation. We were unable to determine whether this method worked in practice because at the time of writing the PDA was being augmented with predictive models and thus not yet evaluated in practice. 


\subsubsection{Barriers and facilitators to SDM and PDAs from the clinician perspective (research questions 2 and 3 )}

Chapter 4 explored the barriers to introducing the prostate cancer PDA developed in Chapter 3 into clinical practice and generated possible strategies to ease implementation. We interviewed prostate cancer specialists and general practitioners (GPs) in the South Limburg region to gain insights into these two topics. It became apparent that prostate cancer patients making a treatment decision needed additional support across the treatment trajectory. The existing information provision was not sufficient as many patients tended to return to their GP after diagnosis for reassurance and advice. Our participants suggested three structural facilitators that could support patient engagement in SDM: multidisciplinary consultations, a formal role for GPs in the form of a post-diagnosis consultation, and introduction of a PDA through oncology nurses.

The preceding chapters covered SDM in prostate cancer, a preferencesensitive condition. In Chapters 5 and $\mathbf{6}$ we explored the potential for SDM in the lung cancer trajectory, a disease group that is not considered preference-sensitive. In Chapter 5, we presented the results of a mixed methods study in which we examined lung cancer patients' treatment decisions and the level of deviation from multidisciplinary tumor board (MTB) recommendations. The qualitative component of the study involved interviews with lung cancer specialists on the nature of decision-making and attitudes towards clinical decision support systems (CDSSs) supported by artificial intelligence (AI). Clinicians felt that the more heavily guideline-driven nature of lung cancer distinguishes it from other cancer types and the fact that there is usually one treatment option that is clinically superior to the alternatives limits the scope for SDM. We found a deviation rate from MTB recommendations of $10.9 \%$, indicating that the majority of patients do not deviate from clinical recommendations, and among patients who do deviate, just under $86 \%$ prefer a less intensive treatment.

The theme of SDM in lung cancer was explored further in Chapter 
6 in the context of treatment decisions in terminal extensive-stage small-cell lung cancer (ES-SCLC). One in ten SCLC patients experience metastasis of the tumors to the brain at the time of diagnosis, around half develop it within two years and up to $80 \%$ have brain tumors at the time of death [1]. In order to reduce this risk, ES-SCLC patients are given prophylactic cranial irradiation (PCI). However, PCI brings with it side-effects such as nausea, fatigue, and potential cognitive decline over the long-term [2]. Recent studies call for the patient's preference to be taken into account in the decision for PCI [3]. In this chapter, we implemented a SDM training for radiation oncologists specialized in lung cancer and evaluated the subsequent level of SDM in 25 clinician-patient consultations. We found that despite a strong preference for taking an active participative role in the process, most ES-SCLC patients faced considerable decisional conflict in making their decision. The main source of this decision conflict was difficulty in weighing the harms and benefits of PCI. We concluded that although it may not be possible to eliminate decisional conflict entirely, prognostic models focused on the PCI in ES-SCLC may support patients and clinicians in evaluating the expected benefit of PCI relative to the expected survival time.

The final chapter (Chapter 7) presented an example of successful SDM implementation at Vejle Hospital in Denmark. We explored clinician perspectives of barriers and facilitators to SDM and PDA implementation through qualitative semi-structured interviews. The main conclusion was that implementation is a continuous multi-stage process with barriers at each stage, and it is important to align possible facilitators as part of a coherent strategy. For instance, there is a natural skepticism and resistance towards implementing any new innovation which involves a change in behavior and daily routines. Therefore, it was important to set the stage for SDM by involving clinicians in the training and PDA development process as this ownership gave clinicians autonomy and allowed them to develop a common language and culture around SDM with their peers. 


\subsubsection{Optimizing care pathways for SDM (research question 4)}

Current literature on SDM tends to focus on the interaction between clinician and patient, however organizational factors such as culture, available resources, and clinical workflows also have a large impact on implementation success. In Chapter 4 it became apparent that modifying the prostate cancer treatment trajectory can provide better opportunities for SDM as well as make it easier to introduce PDAs at the right moment. The modifications suggested by our participants (multidisciplinary clinics and explicitly defined roles for GPs and nurses) require a comprehensive review of the current trajectory conducted by hospital administrators and leaders. SDM initiatives must therefore be an organizational priority, and strong leadership plays a key role in coordinating implementation efforts across the organization and improving quality of care [4]. Simultaneously, empowering clinicians by giving them a degree of autonomy in SDM agendas may make implementation efforts more effective, as demonstrated by our findings in Chapter 7. Examples include inviting clinician participation and feedback in goal setting and developing SDM training activities and PDAs.

In Chapter 7 we also found that the format of decision support was a significant factor in the implementation; in contrast to web-based PDAs for patients to use at home, paper PDAs in the form of decision cards were brought into the consultation to be used by both patients and clinicians. These PDAs consisted of a series of cards corresponding to the five main steps of SDM (inviting the patient's participation, clarifying the decision to be made, identifying what matters to the patient, weighing the pros and cons of each treatment option, and making a decision if the patient is ready). Clinicians mentioned that using these PDAs in the consultations on a continuous basis helped structure the consultation and solidify the principles of the SDM training. Evaluations by the hospital also found that the length of the consultations did not significantly increase and the variability in consultation lengths decreased, suggesting that this structured PDA format helped to standardize the SDM conversation. 


\subsection{Strengths and limitations}

In this research we sought out the perspectives of a variety of stakeholders: patients, urologists, oncologists from several disease areas, radiation oncologists, nurses, and GPs. This enabled us to gain a comprehensive view of the barriers and facilitators to SDM implementation. We also explored the possibilities for SDM in lung cancer treatment decisions, a disease area that is not commonly studied in this context. Till date, the majority of studies about SDM in lung cancer focus on screening decisions rather than treatment decisions.

This thesis primarily used qualitative methods to address the research questions. In contrast to quantitative studies, which are concerned with proving hypotheses and cause and effect, qualitative research is concerned with the 'how' and 'why'. It is ideally suited to investigate the motivations behind certain human behaviors and generate hypotheses for further testing. However, qualitative research is inherently context-specific and this limits the generalizability of its findings. Therefore, although our studies add to the current literature by providing specific examples of SDM and PDA implementation and practical suggestions for implementation based on clinician perspectives, these suggestions need to be tested in different contexts to provide a clearer picture of what is needed for successful implementation.

In addition, qualitative research is subjective and there is a risk of interpretation bias. To the extent possible we have tried to limit this by having participants verify their interview transcripts to ensure that their statements were captured accurately, and by coding with multiple researchers. Since our studies' sample sizes are small and mostly limited to one clinic, care should be taken when extrapolating our results to different contexts. Nevertheless, the fact that our findings largely correspond with existing literature indicates that some level of generalizability has been achieved.

Three studies (Chapters 3, 4, and 6) made use of survey data in the form of usability questionnaires, SDM-related questionnaires, and patient-reported outcome measures (PROMs). These tools offer 
greater reliability since the questionnaires used were standardized and validated instruments. Nevertheless, there is also the possibility of a ceiling effect, in which participants' responses tend to cluster towards the positive/higher end of the measurement scale. The ceiling effect tends to be more pronounced in Likert-scaled instruments measuring patient satisfaction [5]. For instance, ceiling effects have been observed in the SDM-Q-9 questionnaire [6]. Some patients might rate their satisfaction more highly due to social desirability [7]. One method to account for the ceiling effect is top score analysis, in which participants are classified into one of two groups depending on whether they gave the highest scores in their responses, with the aim being to induce variation in scores for more meaningful comparison [8]. This method has not been widely used in the SDM literature and it is unclear to what extent it is applicable in SDM instruments. An alternative solution to account for the ceiling effect and social desirability would be to enlist an independent observer to rate the level of SDM in consultations using the OPTION instrument [9].

\subsection{Challenges and opportunities for SDM implementation}

Having answered the research questions by means of the individual studies, we will now consider our findings in the context of the current literature. In the studies described in this thesis it became apparent that SDM involves not only patients and clinicians but also includes family members, other healthcare professionals, such as nurses and GPs, as well as systemic change through actors at the policy level such as health insurers, patient organizations, and policy-makers. These entities are represented in Figure 8.1, which depicts the clinical encounter between patients, clinicians and nurses as the core of SDM, facilitated by various types of decision aids and the actions of the hospital system, such as the multidisciplinary tumor board (MTB) and administrators who formulate the care pathway. Outside the clinical context, 
GPs and patient organizations play an important supporting role at various points in the treatment trajectory, along with health insurers and policy-makers who influence incentive structures. In the following section we reflect on the challenges and opportunities for SDM at these various levels.

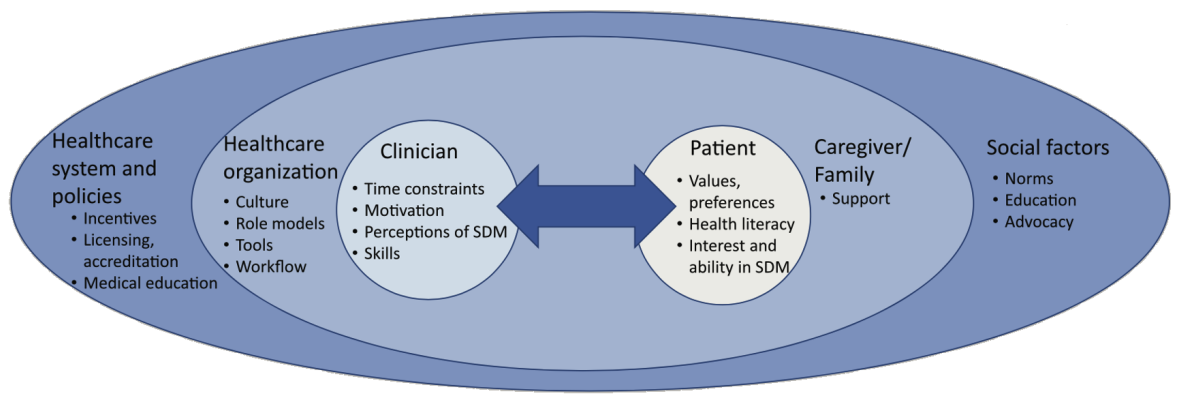

Figure 8.1: A representation of the main entities that participate in the healthcare system [8]

\subsubsection{The clinical encounter}

Although SDM is the process of collaboration between patients and their clinicians, the clinician plays the pivotal role in inviting the patient into the SDM process. Therefore, it is critical for clinicians to have a clear understanding of what SDM entails. The term 'SDM' means different things to different people [9]. Over the years it has been defined as the process of two-way communication between patient and clinician [10], the process of reaching an agreement on the best treatment based on clinical evidence and patient preferences [11], or simply the process of involving the patient in deliberation. Although these definitions appear similar, they are easily conflated with the ideas of informed decision-making or patient-centeredness in general, leading to the belief among many clinicians that they practice SDM despite evidence suggesting otherwise [12]. A situation in which clinicians and hospital administrators have divergent views on what SDM actually 
means and what the end goals are can thus be a significant implementation barrier [13].

In our own studies we observed that most clinicians were aware of SDM as a paradigm but had varying definitions of what it entails. To some, SDM meant providing patients with as much information as possible and leaving the final treatment choice up to them. Others felt it was important for the clinician to suggest the most ideal treatment based on their clinical background. This was observed across disciplines (oncologists, radiation oncologists) in both the prostate and lung cancer studies we conducted in the Netherlands. It was less prevalent in the Danish clinicians we interviewed as they had greater exposure to SDM in practice. However, in the initial development phase of the hospital's SDM effort the Center observed similar perceptions from clinicians, i.e. SDM means providing as much information as possible and that this is something clinicians already do [14].

This lack of clarity can lead to the misconception that SDM can be achieved by simply providing patients with PDAs [15]. Although PDAs have been associated with improvements in patient knowledge, conflating SDM with PDAs neglects the crucial distinction that SDM is a process while PDAs are informational tools meant to support this process. While information is a necessary component of decision-making, patients can become overwhelmed by the amount of complex information they are required to absorb, especially if not all of it is relevant to their situation [16]. Furthermore, the population of cancer patients is heterogeneous in terms of age, educational and cultural background, values, and preferences. Informational needs differ from patient to patient, and the presence of the clinician is crucial in customizing clinical information to suit individual needs.

Successful SDM therefore requires clinicians to adopt a distinct set of behaviors, best encapsulated in the OPTION-12 instrument [17]. This instrument is meant to be used by an external observer who witnesses the clinical interaction and rates the extent to which the clinician involves the patient on twelve dimensions using a five-point scale. A 
systematic review of OPTION scores among clinicians from various disciplines, has found that average OPTION scores tend to be low, with the least frequently observed behavior being assessing the patient's preferred approach and level of involvement (items 3 and 10) [12].

This pattern has been observed within the oncology domain as well and is exacerbated by the fact that little is known about how patients construct their preferences [18]. Evidence suggests that patient preferences are not stable but dynamic according to the changing circumstances of the patient's context [19]. Preferences take form and change as patients gain new information [20]. It is well-known from the field of behavioral science that people's preferences are affected by how information is presented to them, a phenomenon known as the framing effect. For example, people's choices about a treatment option change depending on whether the same information is presented in a positive frame ('lives saved') or a negative frame ('lives lost') [21]. Similarly, patients have a preference for treatments that are presented in terms of relative risk rather than absolute risk [22].

Cognitive bias and the use of heuristics are not limited to patients. There is evidence of cognitive bias among clinicians, the most common ones being anchoring, the framing effect, and information bias [23]. As with patients, a preference for relative risk over absolute risk has been observed in clinicians and medical directors, even when the relative risk option was suboptimal [24]. In $71.4 \%$ of the studies reviewed, these biases were linked with medical errors, such as diagnostic inaccuracies [23]. Since such errors can reduce patients' quality of life and worsen outcomes, it is imperative to understand and account for the effect of cognitive biases in decision-making.

Although the literature in debiasing participants in clinical decisionmaking is sparse, several methods have been put to the test. Inviting clinicians to slow down, consider alternative interpretations of their initial hypotheses, and explain their own thought process (so-called 'metacognition') has shown encouraging results in reducing errors and 
certain cognitive biases $[25,26]$. Training in health literacy and statistical concepts have the most positive effect on cognitive biases that are related to statistics (such as interpreting risks) but not on other biases. Less effective methods include strategies to educate clinicians on cognitive biases [27]. When designing PDAs, it is important to be aware of how the framing of treatment options can affect people's interpretations.

There is another important participant in most clinical interactions whose perspective we have not explored in this thesis: the patient's caregiver or family member(s). We have established that patients and clinicians differ in how they evaluate treatment options, however evidence is emerging that caregivers too evaluate treatment options differently from patients. As an example, caregivers show stronger preferences towards more aggressive treatments and are willing to pay three times more for a treatment that extends the patient's life by one year than patients themselves $[28,29]$. The role of the caregiver in SDM has not been explored in the literature and must be taken into account [30].

\subsubsection{The organizational level}

The clinical interaction is only one component of the cancer treatment trajectory. The full trajectory consists of diagnosis, treatment decisions, treatment delivery, follow-up care, and possibly palliative care. Multiple decisions are made at these different points and involve GPs, oncology nurses, collaboration between groups of clinicians in MTBs, and hospital administrators. SDM therefore requires an understanding of the trajectory, clinician workflow, and provision of the appropriate decision support tools.

Throughout this research, it became apparent that current patient trajectories can be a barrier to SDM. In our study of the prostate cancer trajectory, we found that there was insufficient time to engage in the full process of SDM. This was further confirmed with the finding 
that many patients return to their GP for confirmation and advice. In more rapidly progressing diseases like lung cancer, this issue is even more acute; the trajectory is intentionally designed to reduce the time between diagnosis and the start of the treatment, because the fastgrowing nature of lung tumors makes it advantageous to begin treatment as soon as possible. A second reason is to reduce the amount of time that the patient must wait in uncertainty between receiving their diagnosis and learning what the next course of action will be [31]. Although designed with the best of intentions, these systems leave little room for a detailed consideration of the patient's perspective. Patients' ability to take part in SDM can also be affected indirectly by the fragmented nature of most patient trajectories, where patients must visit multiple specialists in different locations. The perception that clinician workloads are hectic can lead patients to hesitate in asserting their perspectives and wishes in order to not impose on busy clinicians [32].

One proposed solution is that SDM should be extended across the trajectory and not just at the treatment decision point [33]. For instance, in the Netherlands, post-diagnosis time-out consultations with GPs have been proposed so that patients can clarify their values and deliberate on their treatment options in a setting where long-term trust has been established [34]. The need for a greater role for the GP in the cancer care trajectory has prompted one study to systematically implement a designated time-out consultation. These measures can help patients feel a sense of continuity and consistency throughout their treatment journey. This experience of seamless care from a team of clinicians and hospital staff can build trust that is fundamental in patient participation and SDM [35].

A practical method to improve care continuity is patient journey mapping, also referred to as process mapping [36]. This is the process of tracing the path that a typical patient would take, usually from entry into the hospital system to discharge, and taking note of various metrics such as the number of interactions patients have with staff (receptionists, nurses, clinicians), the length of waiting times, etc. The purpose is to visualize the patient's journey through the hospital system and identify areas for quality improvement. 
Another potential use for patient journey mapping that has been less explored is to determine the optimal way to integrate clinical decision support systems into the treatment trajectory [37]. We have previously described the proliferation of clinical data from various sources such as EHRs, outputs from PDAs, and increasingly even wearable technologies. Integrating clinical and patient data into CDSSs offers tremendous potential to tailor treatments to individual patients so that they receive the treatment that is most likely to benefit them. This requires that the right information can be accessed at the right time [38]. Process mapping can help ensure that CDSSs are better integrated into the clinical workflow and are able to provide clinicians and patients with the information required to engage in SDM.

Integrating decision tools into the clinical workflow, while a complex undertaking, is one of the easier challenges to tackle. The greater challenge is building trust in CDSSs. Clinician mistrust in CDSSs comes in several forms: lack of transparency, a perceived threat to clinician autonomy, lack of actionability, and the inability of a CDSS to take contextual information about a patient into account in the way a human would [39]. Lack of trust is even more pronounced for CDSSs that are augmented with artificial intelligence/machine learning (AI/ML) algorithms. These AI-CDSSs are sometimes by their very nature "black boxes', since the process through which they come to certain risk estimates or recommendations may be invisible or unclear. These factors may explain why the majority of CDSSs fail to be adopted in routine clinical practice [40].

Recent literature from the philosophy domain proposes that the concept of trust should be reserved for "reciprocal relations between beings with agency" [41,42]. In other words, the extent to which clinicians are able to trust CDSSs is moderated by the extent to which they can trust CDSS developers [43]. Developers can build trust in CDSSs, AI or otherwise, by including clinicians in the development process to the extent possible. This entails consulting with clinicians about specific use cases or situations in which decision support is valuable and desired. In addition, developers can build trust along three core di- 
mensions: correctness about the CDSS's accuracy, providing assurance that the CDSS is regularly updated, and that it complies with regulatory standards and legal requirements [43].

\subsubsection{The healthcare system/policy-level}

Throughout the course of this thesis, the theme of incentivising SDM through various external channels emerged. First, reliability and balanced information provided by PDAs was a priority for the clinicians we interviewed. As PDAs can potentially influence treatment choices, there needs to be a way to ensure patient safety. Current criteria such as the IPDAS guidelines provide a checklist of criteria to guide PDA development to maximize quality, reliability, and user acceptance. However, this process is prescriptive and may not guarantee a generally accepted measure of quality. External certification such as $\mathrm{CE}$ marking may support implementation by ensuring that the PDA meets well-established (international) quality standards. This in turn may provide an added incentive for clinicians, hospital administrators, patients and insurers to use them in practice.

A second challenge to SDM implementation concerns the manner in which the healthcare system is structured. In the Netherlands, the government and certain medical associations have established minimum volume standards for the number of procedures a hospital must perform [44]. These standards are intended to improve care quality, based on the assumption that a greater volume of procedures conducted improves clinician expertise. However, this system can also have unintended consequences, not least for SDM. These volume standards give for instance surgical oncologists a greater incentive to perform surgeries, and our findings indicated that these clinicians are the key decision-makers in the current trajectory. Thus it is likely that a disproportionate number of patients undergo surgery, and indeed this pattern has been observed both in the United States as well as Europe $[45,46]$. 
Healthcare insurers may play a role in aligning these incentives. Dutch healthcare insurers are becoming more knowledgeable about SDM and supporting methods to ensure that patients receive treatments tailored to their profile and preferences [47]. Insurers can incentivize patients and clinicians to take a more collaborative role in treatment decisions, for instance by paying clinicians for the use of PDAs or making their use a prerequisite for reimbursing certain procedures. This may be particularly relevant in prostate cancer management, where multiple treatment options offer comparable clinical outcomes and yet there is still a tendency for patients to undergo surgery. Inclusion of a reimbursement code for the PDA can help standardize the process and speed up its usage, particularly since insurers see evidence of PDA use as one way to make the SDM process more visible and are heavily influencing hospitals to implement them [47].

\subsection{Conclusion}

SDM is widely perceived to be a key component of patient-centered care, yet there are numerous challenges in its implementation. Successful SDM depends on the understanding and willing participation from patients, clinicians, and other stakeholders. In this thesis, we gained a deeper understanding of the motivations, needs, perspectives, and manner of working of these groups. We found that most of the barriers to implementation tend to be psychological/behavioural and structural/organizational. Encouragingly, we observed willingness from patients and clinicians alike to take part in SDM. The gap between intention and action can be narrowed by creating organizational structures and cultures that support collaboration, not only between patients and clinicians but also developers, hospital administrators, primary care providers, and the wider healthcare system. 


\section{References}

1. Elwyn G, O'Connor A, Stacey D, Volk R, Edwards A, Coulter A, et al. Developing a quality criteria framework for patient decision aids: online international Delphi consensus process. BMJ 2006;333:417.

2. Tong A, Sainsbury P, Craig J. Consolidated criteria for reporting qualitative research (COREQ): a 32-item checklist for interviews and focus groups. Int. J. Qual. Health Care 2007;19:349-57.

3. Voutilainen A, Pitkäaho T, Kvist T, Vehviläinen-Julkunen K. How to ask about patient satisfaction? The visual analogue scale is less vulnerable to confounding factors and ceiling effect than a symmetric Likert scale. J. Adv. Nurs. 2016;72:946-57.

4. Scholl I, Kriston L, Dirmaier J, Härter M. Comparing the nine-item Shared Decision-Making Questionnaire to the OPTION Scale - an attempt to establish convergent validity. Health Expect. Int. J. Public Particip. Health Care Health Policy 2015;18:137-50.

5. Chewning B, Bylund CL, Shah B, Arora NK, Gueguen JA, Makoul G. Patient preferences for shared decisions: a systematic review. Patient Educ. Couns. 2012;86:9-18.

6. Barr PJ, Thompson R, Walsh T, Grande SW, Ozanne EM, Elwyn G. The Psychometric Properties of CollaboRATE: A Fast and Frugal Patient-Reported Measure of the Shared Decision-Making Process. J. Med. Internet Res. 2014;16:e2.

7. Elwyn G, Hutchings H, Edwards A, Rapport F, Wensing M, Cheung W, et al. The OPTION scale: measuring the extent that clinicians involve patients in decision-making tasks. Health Expect. 2005;8:34-42.

8. Tan ASL, Mazor KM, McDonald D, Lee SJ, McNeal D, Matlock DD, et al. Designing Shared Decision-Making Interventions for Dissemination and Sustainment: Can Implementation Science Help Translate Shared Decision Making Into Routine Practice? MDM Policy Pract. 2018;3:2381468318808503.

9. Moumjid N, Gafni A, Brémond A, Carrère M-O. Shared decision making in the medical encounter: are we all talking about the same thing? Med. Decis. Mak. Int. J. Soc. Med. Decis. Mak. 2007;27:539-46.

10. Charles C, Gafni A, Whelan T. Shared decision-making in the medical 
encounter: What does it mean? (or it takes at least two to tango). Soc. Sci. Med. 1997;44:681-92.

11. Joosten EA, DeFuentes-Merillas L, De Weert G, Sensky T, Van Der Staak C, de Jong CA. Systematic review of the effects of shared decision-making on patient satisfaction, treatment adherence and health status. Psychother. Psychosom. 2008;77:219-26.

12. Couët N, Desroches S, Robitaille H, Vaillancourt H, Leblanc A, Turcotte $\mathrm{S}$, et al. Assessments of the extent to which health-care providers involve patients in decision making: a systematic review of studies using the OPTION instrument. Health Expect. 2015;18:542-61.

13. Lloyd A, Joseph-Williams N, Edwards A, Rix A, Elwyn G. Patchy 'coherence': using normalization process theory to evaluate a multi-faceted shared decision making implementation program (MAGIC). Implement. Sci. 2013;8:102.

14. Steffensen KD. Re: The responses to the "cancer drugs scandal" must fully in- volve patients-An essay by Tessa Richards [Internet]. 2017;Available from: https://www.bmj.com/content/359/bmj.j4956/rr-7

15. Légaré F, Thompson-Leduc P. Twelve myths about shared decision making. Patient Educ. Couns. 2014;96:281-6.

16. Josfeld L, Keinki C, Pammer C, Zomorodbakhsch B, Hübner J. Cancer patients' perspective on shared decision-making and decision aids in oncology. J. Cancer Res. Clin. Oncol. 2021;147:1725-32.

17. Ahmad M, Abu Tabar N, Othman EH, Abdelrahim Z. Shared Decision-Making Measures: A Systematic Review. Qual. Manag. Health Care 2020;29:54-66.

18. Katz SJ, Belkora J, Elwyn G. Shared decision making for treatment of cancer: challenges and opportunities. J. Oncol. Pract. 2014;10:206-8.

19. Elwyn G, Miron-Shatz T. Deliberation before determination: the definition and evaluation of good decision making. Health Expect. Int. J. Public Particip. Health Care Health Policy 2010;13:139-47.

20. Lichtenstein S, Slovic P, editors. The Construction of Preference [Internet]. Cambridge: Cambridge University Press; 2006 [cited 2020 Dec 19]. Available from: http://ebooks.cambridge.org/ref/id/CBO9780511618031 
21. Tversky A, Kahneman D. The framing of decisions and the psychology of choice. science 1981;211:453-8.

22. Blumenthal-Barby JS, Krieger H. Cognitive Biases and Heuristics in Medical Decision Making: A Critical Review Using a Systematic Search Strategy. Med. Decis. Making 2015;35:539-57.

23. Saposnik G, Redelmeier D, Ruff CC, Tobler PN. Cognitive biases associated with medical decisions: a systematic review. BMC Med. Inform. Decis. Mak. 2016;16:138.

24. Mezzio DJ, Nguyen VB, Kiselica A, O'Day K. Evaluating the Presence of Cognitive Biases in Health Care Decision Making: A Survey of U.S. Formulary Decision Makers. J. Manag. Care Spec. Pharm. 2018;24:1173-83.

25. Mussweiler T, Strack F, Pfeiffer T. Overcoming the inevitable anchoring effect: Considering the opposite compensates for selective accessibility. Pers. Soc. Psychol. Bull. 2000;26:1142-50.

26. Gilovich T. How we know what isn't so: the fallibility of human reason in everyday life. New York [Great Britain]: Free Press; 1991.

27. O'Sullivan E, Schofield S. Cognitive bias in clinical medicine. J. R. Coll. Physicians Edinb. 2018;48:225-32.

28. Ozdemir S, Finkelstein EA. Cognitive Bias: The Downside of Shared Decision Making. JCO Clin. Cancer Inform. 2018;1-10.

29. Malhotra C, Farooqui MA, Kanesvaran R, Bilger M, Finkelstein E. Comparison of preferences for end-of-life care among patients with advanced cancer and their caregivers: A discrete choice experiment. Palliat. Med. 2015;29:842-50.

30. Hamann J, Heres S. Why and How Family Caregivers Should Participate in Shared Decision Making in Mental Health. Psychiatr. Serv. 2019;70:418-21.

31. Perfors IAA. Improving primary care involvement in cancer care.: [Internet]. 2021 [cited 2021 Jun 22];Available from: https://dspace.library.uu.nl/handle/1874/401923

32. Berry LL, Danaher TS, Beckham D, Awdish RLA, Mate KS. When Patients and Their Families Feel Like Hostages to Health Care. Mayo Clin. Proc. 2017;92:1373-81. 
33. Roodbeen R, Vreke A, Boland G, Rademakers J, van den Muijsenbergh $\mathrm{M}$, Noordman J, et al. Communication and shared decision-making with patients with limited health literacy; helpful strategies, barriers and suggestions for improvement reported by hospital-based palliative care providers. PLOS ONE 2020;15:e234926.

34. de Wit NJ. A "time out consultation" in primary care for elderly patients with cancer: Better treatment decisions by structural involvement of the general practitioner. Eur. J. Cancer Care (Engl.) 2017;26:e12711.

35. Berry LL, Awdish RLA, Letchuman S, Steffensen KD. Trust-Based Partnerships Are Essential - and Achievable - in Health Care Service. Mayo Clin. Proc. 2021;S0025619621002652.

36. Trebble TM, Hansi N, Hydes T, Smith MA, Baker M. Process mapping the patient journey: an introduction. Bmj 2010;341.

37. Borycki EM, Kushniruk AW, Wagner E, Kletke R. Patient journey mapping: Integrating digital technologies into the journey. :16.

38. Adoption and use of Electronic Medical Records and ePrescribing [Internet]. In: Health at a Glance: Europe 2018. OECD; 2018 [cited 2021 Jun 25]. page 192-3.Available from: https://www.oecd-ilibrary.org/social-issuesmigration-health/health-at-a-glance-europe-2018/adoption-and-use-ofelectronic-medical-records-and-eprescribing health glance eur-2018-56-en

39. Ford E, Edelman N, Somers L, Shrewsbury D, Lopez Levy M, van Marwijk $\mathrm{H}$, et al. Barriers and facilitators to the adoption of electronic clinical decision support systems: a qualitative interview study with UK general practitioners. BMC Med. Inform. Decis. Mak. 2021;21:193.

40. Greenhalgh T, Wherton J, Papoutsi C, Lynch J, Hughes G, A'Court C, et al. Beyond Adoption: A New Framework for Theorizing and Evaluating Nonadoption, Abandonment, and Challenges to the Scale-Up, Spread, and Sustainability of Health and Care Technologies. J. Med. Internet Res. 2017;19:e367.

41. Hatherley JJ. Limits of trust in medical AI. J. Med. Ethics 2020;46:478-81.

42. O'Neill O. Linking trust to trustworthiness. Int. J. Philos. Stud. 2018;26:293-300. 
43. Jones C, Thornton J, Wyatt JC. Enhancing trust in clinical decision support systems: a framework for developers. BMJ Health Care Inform. 2021;28:e100247.

44. Mesman R, Faber MJ, Westert GP, Berden B. Exploring Dutch surgeons' views on volume-based policies: a qualitative interview study. J. Health Serv. Res. Policy 2018;23:185-92.

45. Kim SP, Gross CP, Nguyen PL, Nguyen PY, Smaldone MC, Thompson RH, et al. Specialty bias in treatment recommendations and quality of life among radiation oncologists and urologists for localized prostate cancer. Prostate Cancer Prostatic Dis. 2014;17:163-9.

46. Borras JM, Lievens Y, Grau C. The need for radiotherapy in Europe in 2020: Not only data but also a cancer plan. Acta Oncol. 2015;54:1268-74.

47. van der Weijden T, Post $H$, Brand PLP, van Veenendaal $H$, Drenthen $T$, van Mierlo LA, et al. Shared decision making, a buzz-word in the Netherlands, the pace quickens towards nationwide implementation... Z Z. Evidenz Fortbild. Qual. Im Gesundheitswesen 2017;123-124:69-74. 


\section{Summary}

Shared decision-making (SDM) is the process of collaboration between patients and clinicians to choose a treatment that takes into account both the best available clinical evidence as well as the patient's personal values and preferences. SDM is rapidly gaining attention in the medical community as it offers the possibility of greater patient satisfaction, better outcomes, and more efficient use of resources as a result of patients receiving the treatment that is most suited to them. SDM is particularly relevant in certain cancer types where there are multiple treatment options without one single clinically superior treatment. These are named 'preference-sensitive' conditions as the ideal treatment then depends on the patient's preferences. Bringing patients into the decision-making process involves providing them with relevant, accurate, and easy to understand clinical information as well as engaging in a dialogue to elicit their values and what matters to them. This requires clinicians to become familiar with new skills and information tools. Implementing these tools and skills is challenging due to unfamiliarity, busy clinician workloads, and the tendency to revert to traditional ways of conducting consultations. In this thesis, we present the challenges and opportunities to patient participation under SDM.

Chapter 1 provides the background of the thesis. It begins with a summary of the changing nature of decision-making in oncology due to three trends: the rising incidence of cancer due to demographic and lifestyle changes, rapid advances in treatment modalities, and the Information Age which gives clinicians and patients unprecedented access to clinical data and knowledge about diseases and treatments. These trends necessitate greater collaboration between patients and their clinicians in order to select the best treatment suited to the individual patient's values and preferences. This collaboration, known as shared decision-making (SDM), is often supported by patient decision aids (PDAs) and clinical decision support systems (CDSSs) - tools that 
summarize complex clinical information and present the treatment alternatives in a systematic and user-friendly format. The implementation of SDM and these tools often meet with challenges such that these innovations are yet to become part of usual care. This thesis explores the following questions:

1. How to develop a PDA that fulfills user requirements and clinical standards?

2. What do clinicians perceive as barriers and facilitators to implementing SDM in prostate cancer/preference-sensitive conditions?

3. How do these barriers and facilitators manifest in less preference-sensitive conditions, such as lung cancer?

4. How can SDM and PDA delivery be optimized to account for the barriers and facilitators?

In Chapter 2, we take a closer look at the process of developing a PDA. We review the current literature in order to summarize the definition, goals, benefits, and limitations of PDAs. A PDA is any tool, digital or in a printed format, that provides an overview of a disease, its treatment options, and a comparison between the options. Many PDAs also include questions for the patient to answer that help clarify what matters to them. Well-informed patients can then take part in a SDM consultation and deliberate on their options with their clinician. Indeed, evidence from randomized controlled trials (RCTs) shows that patients who use PDAs display greater knowledge about their disease, are more accurately able to interpret the risks associated with their treatment options, and tend to take a more active role in making treatment decisions. However, there is mixed evidence on the costeffectiveness of PDAs and little consensus on the best way to ensure that they become part of routine care. User-centered design methods can increase the likelihood that a PDA is accurate, acceptable, and user-friendly. The challenge remains to integrate PDAs into the clinical pathway in the face of busy workloads, low health literacy, and the need for improved communication between patients and clinicians. 
Chapter 3 describes the process of developing a PDA for prostate cancer patients based on a user-centered design methodology. We built a PDA prototype and refined it over four rounds of semi-structured interviews and think-aloud evaluations with former prostate cancer patients, specialists (urologists, radiation oncologists, and oncology nurses), and general practitioners. The purpose of these evaluations was to understand patients' informational needs and clinicians' perspectives on the accuracy of the PDA. Having established the content of the PDA over the first four rounds, the fifth round focused on improving usability based on heuristic evaluation with usability experts and preference testing with healthy volunteers who had no history of prostate cancer. The benefit of our iterative process was that it provided flexibility to continuously improve the PDA through incremental changes while remaining in line with users' expectations. The challenge lay in establishing consensus among different users as our process produced a rich set of perspectives and opinions.

In Chapter 4 we determine barriers and facilitators to implementing SDM with a digital PDA in the prostate cancer trajectory from the perspective of clinicians (urologists, radiation oncologists, oncology nurses, and general practitioners (GPs)). We chose to interview clinicians because they conduct the decision-making process, from GPs who make the initial referral to a prostate cancer specialist for diagnostic tests to specialists who present the diagnosis and treatment options to the patient. Therefore, implementing a PDA as part of a SDM process requires the cooperation of a variety of clinicians. We found that providing patients with accurate and easy to understand clinical information is only one aspect of the process. The other factor is a trusting environment which provides patients with enough time to consider all their options and share perspectives with a multitude of clinicians, both specialists and GPs. As a result, we found three facilitators that may support SDM implementation: post-diagnosis time-out consultations with the patient's GP, a multidisciplinary consultation in which the patient could discuss his options with urologists as well as radiation oncologists in order to get a balanced view of all options and their 
implications, and a PDA that is embedded in the patient pathway and introduced to the patient through oncology nurses.

Chapter 5 considers the possibilities for SDM in the lung cancer trajectory, supported by clinical decision support systems (CDSSs). We conduct a mixed methods study consisting of nine interviews with lung cancer specialists from six Dutch hospitals and a cohort study of the treatment decisions of 257 lung cancer patients treated at Maastro. Our findings indicate that current treatment decisions are made in the multidisciplinary tumor board (MTB) and although some clinicians make an effort to elicit the patient's preferences in the subsequent consultation, the treatment indicated by the MTB rarely changes. Clinicians cited the heavy influence of clinical guidelines on lung cancer treatment decisions as a potential barrier to SDM since there is often one clinically superior treatment. Our cohort study revealed that although nearly $90 \%$ of patients follow MTB advice, half of those who deviate do so in favor of less intensive treatments. CDSSs that can predict how an individual patient might respond to a certain treatment can be a valuable addition to the decision-making process. Qualitative interviews with clinicians revealed the biggest barriers to these CDSSs as a lack of trust, a lack of validation studies, and time constraints. More efforts need to be put into validating the prediction models and building them into CDSSs that are embedded in the clinical workflow so that clinicians in the MTB can view the harms and benefits of different treatment options for each patient and discuss these with the patient.

In Chapter 6, we implemented a SDM workshop for radiation oncologists so that they could engage extensive-stage small-cell lung cancer (ES-SCLC) patients in the decision whether to undergo prophylactic cranial irradiation (PCI) as a preventative measure for brain metastasis. We found that most ES-SCLC patients would like to take a participative role in this decision along with their clinician but that they face significant decisional conflict. The main source of this decisional conflict lay in weighing the harms and benefits of PCI in terms of the patient's individual circumstances. We conclude that predictive models that can present the expected survival benefit of PCI based on the 
patient's individual and clinical characteristics may support the SDM consultation.

Chapter 7 presents the experiences of 10 Danish clinicians at a cancer hospital in Denmark where SDM has been implemented with the use of paper PDAs. Since SDM and PDAs had been successfully implemented and evaluated, we were able to gain insights into the challenges before, during, and after the implementation, as well as the strategies used to overcome them. Although the cultural context was different from the previous studies in this thesis, we find that many implementation challenges were common, such as clinician skepticism/resistance and creating sustained change. Several factors helped overcome these barriers. First, clinicians were involved in PDA development and SDM training through the Train-the-trainer method, giving them a feeling of ownership and empowerment. The paper PDAs were structured according to the main steps of the SDM process and were used exclusively within consultations, ensuring that clinicians were supported in putting their training into practice. Organizational culture, mindset, and leadership were considered key facilitators.

Chapter 8 summarizes the findings of the studies presented in the previous chapters in light of the four research questions and considers the strengths and limitations of our research. The main strength is that we engaged a variety of stakeholders both within and outside the clinical interaction in order to gain a holistic view of the current decisionmaking process and which factors may hinder SDM implementation. Although our chosen method of qualitative research provided rich insights into the perspectives of these different stakeholders, a possible limitation is that qualitative research does not prioritize generalizability. Other limitations are that we were not able to evaluate the prostate cancer PDA or perform a controlled study of our SDM implementation in ES-SCLC patients due to the small patient population. Nevertheless, our findings provide an overview of the barriers and facilitators to SDM and PDA implementation at various levels (individual, organizational, and external) and strategies that can be used to ensure that 
clinicians and patients are supported in their collaboration. 


\section{Nederlandse samenvatting}

Gedeelde besluitvorming ('shared decision-making' - ook bekend als $\mathrm{SDM}$ ) is het proces van samenwerking tussen patiënten en clinici om een behandeling te kiezen die rekening houdt met zowel het beste beschikbare klinische bewijs als de persoonlijke waarden en voorkeuren van de patiënt. SDM wint snel aan aandacht in de medische gemeenschap omdat het de mogelijkheid biedt van grotere tevredenheid bij de patiënt, betere resultaten en een efficiënter gebruik van middelen doordat de patiënt de behandeling krijgt die het best bij haar of hem past. SDM is met name relevant bij bepaalde kankertypes waarbij er meerdere behandelingsopties zijn zonder dat er sprake is van één enkele klinisch superieure behandeling. Dit worden "voorkeur gevoelige" aandoeningen genoemd, omdat de ideale behandeling dan afhangt van de voorkeuren van de patiënt. Om patiënten bij het besluitvormingsproces te betrekken, moet hun relevante, nauwkeurige en gemakkelijk te begrijpen klinische informatie worden verstrekt en moet een dialoog worden aangegaan om hun waarden en wat voor hen belangrijk is te achterhalen. Dit vereist dat clinici vertrouwd raken met nieuwe vaardigheden en informatie hulpmiddelen. Het implementeren van deze hulpmiddelen en vaardigheden is een uitdaging vanwege onbekendheid, hoge werkdruk en de neiging om terug te vallen op traditionele manieren van consultvoering. In deze proefschrift presenteren we de uitdagingen en mogelijkheden voor patiëntenparticipatie onder SDM.

Hoofdstuk 1 geeft de achtergrond van het proefschrift. We beginnen met een samenvatting van de veranderende aard van de besluitvorming in de oncologie als gevolg van drie trends: de stijgende incidentie van kanker als gevolg van demografische veranderingen en veranderingen in levensstijl, de snelle vooruitgang in behandelingsmodaliteiten, en het informatietijdperk dat clinici 
en patiënten ongekende toegang geeft tot klinische gegevens en kennis over ziekten en behandelingen. Deze trends maken een grotere samenwerking tussen patiënten en hun clinici noodzakelijk om de beste behandeling te kunnen kiezen die past bij de waarden en voorkeuren van de individuele patiënt. Deze samenwerking, bekend als gedeelde besluitvorming (SDM), wordt vaak ondersteund door keuzehulpen en klinische beslissingsondersteunende systemen ('clinical decision support systems' - CDSS's) - hulpmiddelen die complexe klinische informatie samenvatten en de behandelingsmogelijkheden presenteren in een systematisch en gebruiksvriendelijk formaat. De implementatie van SDM en deze hulpmiddelen is uitdagend, met als gevolg dat deze innovaties nog geen deel uitmaken van de gebruikelijke zorg. Deze thesis onderzoekt de volgende vragen:

1. Hoe kan een keuzehulp worden ontwikkeld die voldoet aan de eisen van de gebruiker en aan de klinische normen?

2. Wat zien clinici als barrières en facilitatoren voor het implementeren van SDM bij prostaatkanker/voorkeur gevoelige aandoeningen?

3. Hoe manifesteren deze barrières en facilitatoren zich bij minder voorkeur gevoelige aandoeningen, zoals longkanker?

4. Hoe kunnen SDM en keuzehulpen worden geoptimaliseerd om rekening te houden met de barrières en facilitators?

In Hoofdstuk 2 gaan we dieper in op het ontwikkelingsproces van een keuzehulp. We bekijken de huidige literatuur om de definitie, doelen, voordelen en beperkingen van keuzehulp samen te vatten. Een keuzehulp is een hulpmiddel, digitaal of papier, dat een overzicht geeft van een ziekte, de behandelingsmogelijkheden en een vergelijking tussen de mogelijkheden. Veel keuzehulpen bevatten ook vragen die de patiënt kan beantwoorden om te verduidelijken wat voor hem belangrijk is. Goed geïnformeerde patiënten kunnen dan deelnemen aan een SDM-consult en met hun arts overleggen over hun opties. Uit gerandomiseerde gecontroleerde onderzoeken 
(RCT's) blijkt dat patiënten die keuzehulpen gebruiken meer kennis over hun ziekte hebben, beter in staat zijn de risico's van hun behandelingsopties te interpreteren en een actievere rol spelen bij het nemen van beslissingen over de behandeling. Er is echter gemengd bewijs over de kosteneffectiviteit van keuzehulpen en er is weinig consensus over de beste manier om ervoor te zorgen dat ze deel gaan uitmaken van de routinezorg. Gebruikersgerichte ontwerpmethoden (user-centered design) kunnen de kans vergroten dat een keuzehulp accuraat, aanvaardbaar en gebruiksvriendelijk is. De uitdaging blijft om keuzehulpen te integreren in het klinische traject, gezien de drukke werklast van de clinicus, de lage gezondheidsgeletterdheid en de behoefte aan betere communicatie tussen patiënten en clinici.

Hoofdstuk 3 beschrijft het proces van het ontwikkelen van een keuzehulp voor prostaatkankerpatiënten op basis van een gebruikersgerichte ontwerpmethodologie. We hebben een keuzehulp prototype gebouwd en het verfijnd in vier ronden van semi-gestructureerde interviews en 'think-aloud' evaluaties met voormalige prostaatkankerpatiënten, specialisten (urologen, radiotherapeut-oncologen, en oncologieverpleegkundigen), en huisartsen. Het doel van deze evaluaties was inzicht te krijgen in de informatiebehoeften van patiënten en de perspectieven van clinici op de nauwkeurigheid van de keuzehulp. Nadat in de eerste vier ronden de inhoud van de keuzehulp was vastgesteld, concentreerde de vijfde ronde zich op het verbeteren van het gebruik op basis van heuristische evaluatie met ervaringsdeskundigen en het bepalen van voorkeuren met gezonde vrijwilligers die geen voorgeschiedenis van prostaatkanker hadden. Het voordeel van ons iteratieve proces was dat het de flexibiliteit bood om de keuzehulp voortdurend te verbeteren door middel van incrementele veranderingen en tegelijkertijd in lijn te blijven met de verwachtingen van de gebruikers. De uitdaging lag in het bereiken van consensus tussen de verschillende gebruikers, aangezien ons proces een rijke verzameling perspectieven en meningen opleverde.

In Hoofdstuk 4 bepalen we barrières en facilitatoren voor het 
implementeren van SDM met een digitale keuzehulp in het prostaatkanker traject vanuit het perspectief van clinici (urologen, radiotherapeut-oncologen (RTO's), oncologieverpleegkundigen, en huisartsen). We kozen ervoor om clinici te interviewen omdat zij het besluitvormingsproces leiden, van huisartsen die de eerste verwijzing naar een prostaatkankerspecialist maken voor diagnostisch onderzoek tot specialisten die de diagnose en behandelingsopties aan de patiënt presenteren. Daarom vereist de implementatie van een keuzehulp als onderdeel van een SDM proces de medewerking van een verscheidenheid aan clinici. We ontdekten dat het verstrekken van accurate en gemakkelijk te begrijpen klinische informatie aan patiënten slechts één aspect van het proces is. De andere factor is een vertrouwensvolle omgeving die patiënten voldoende tijd biedt om al hun opties te overwegen en perspectieven te delen met een veelheid aan clinici, zowel specialisten als huisartsen. Wij vonden bijgevolg drie facilitatoren die de SDM-implementatie kunnen ondersteunen: time-out consultaties met de huisarts na de diagnose, een multidisciplinair consult waarbij de patiënt zijn opties zowel met urologen als met radiotherapeuten kan bespreken om een evenwichtig beeld te krijgen van alle opties en hun implicaties, en een keuzehulp die in het patiëntentraject is ingebed en via oncologieverpleegkundigen aan de patiënt wordt geïntroduceerd.

Hoofdstuk 5 beschouwt de mogelijkheden voor SDM in het longkanker traject, ondersteund door klinische beslissingsondersteunende systemen (CDSSs). We voeren een mixed methods studie uit bestaande uit negen interviews met longkankerspecialisten uit zes Nederlandse ziekenhuizen en een cohort studie van de behandelbeslissingen van 257 longkankerpatiënten behandeld in Maastro. Onze bevindingen geven aan dat de huidige behandelbesluiten worden genomen in de multidisciplinaire overleg (MDO) en hoewel sommige clinici zich inspannen om de voorkeuren van de patiënt te achterhalen in het daaropvolgende consult, verandert de behandeling die door de MTB wordt aangegeven zelden. Artsen noemden de grote invloed van klinische richtlijnen op beslissingen over de 
behandeling van longkanker als een potentiële belemmering voor $\mathrm{SDM}$, omdat er vaak één klinisch superieure behandeling is. Onze cohort studie toonde aan dat hoewel bijna $90 \%$ van de patiënten het MTB advies volgen, de helft van degenen die afwijken dit doen ten gunste van minder intensieve behandelingen. CDSSs die kunnen voorspellen hoe een individuele patiënt op een bepaalde behandeling zou kunnen reageren, kunnen een waardevolle aanvulling zijn op het besluitvormingsproces. Uit kwalitatieve interviews met clinici bleek dat de grootste belemmeringen voor deze CDSSs een gebrek aan vertrouwen, een gebrek aan validatiestudies en tijdgebrek zijn. Onderzoekers moeten ervoor zorgen dat voorspellingsmodellen extern gevalideerd worden en ingebouwd worden in CDSS's die ingebed zijn in de klinische workflow, zodat clinici in het MTB de nadelen en voordelen van verschillende behandelingsopties voor elke patiënt kunnen bekijken en deze met de patiënt kunnen bespreken.

In Hoofdstuk 6 hebben we een SDM workshop voor radiotherapeutoncologen geïmplementeerd, zodat zij patiënten met een uitgebreid stadium van kleincellige longkanker (ES-SCLC) konden betrekken bij de beslissing of zij profylactische hersenbestraling (PCI) zouden ondergaan als preventieve behandeling voor hersenmetastase. We ontdekten dat de meeste ES-SCLC patiënten graag een participatieve rol zouden willen spelen in deze beslissing, samen met hun arts, maar dat ze een aanzienlijk beslissingsconflict ervaren. De belangrijkste bron van dit beslissingsconflict lag in de afweging van de voor- en nadelen van PCI in het licht van de individuele omstandigheden van de patiënt. Wij concluderen dat voorspellende modellen die het verwachte overlevingsvoordeel van PCI kunnen weergeven op basis van de individuele en klinische kenmerken van de patiënt, het SDM-overleg kunnen ondersteunen.

Hoofdstuk 7 presenteert de ervaringen van 10 Deense clinici in een kankerziekenhuis in Denemarken waar SDM is geïmplementeerd met het gebruik van papieren keuzehulpen. Omdat SDM en keuzehulpen succesvol waren geïmplementeerd en geëvalueerd, waren we in staat om inzicht te krijgen in de uitdagingen voor, 
tijdens en na de implementatie, evenals de strategieën die werden gebruikt om deze te overwinnen. Hoewel de culturele context verschilde van de eerdere studies in dit proefschrift, vonden we dat veel implementatie-uitdagingen gemeenschappelijk waren, zoals scepticisme/weerstand van clinici en het creëren van duurzame verandering. Verschillende factoren hielpen om deze barrières te overwinnen. Ten eerste werden de clinici betrokken bij de ontwikkeling van de keuzehulpen en de opleiding in SDM via de train-de-trainer methode, waardoor ze een gevoel van eigenaarschap en empowerment kregen. De keuzehulpen waren gestructureerd volgens de belangrijkste stappen van het SDM-proces en werden uitsluitend gebruikt tijdens consultaties, zodat de clinici werden ondersteund bij het in de praktijk brengen van hun SDM training. Organisatiecultuur, mentaliteit en leiderschap werden beschouwd als de belangrijkste facilitatoren.

Hoofdstuk 8 geeft een samenvatting van de bevindingen in de vorige hoofdstukken in het licht van de vier onderzoeksvragen en gaat in op de sterke punten en beperkingen van ons onderzoek. Een sterk punt is dat we een verscheidenheid aan stakeholders binnen en buiten de klinische interactie hebben betrokken om een holistisch beeld te krijgen van het huidige besluitvormingsproces en welke factoren de implementatie van SDM kunnen belemmeren. Hoewel de door ons gekozen methode van kwalitatief onderzoek rijke inzichten verschafte in de perspectieven van deze verschillende stakeholders, is een mogelijke beperking dat kwalitatief onderzoek minder prioriteit geeft aan generaliseerbaarheid. Andere beperkingen zijn dat we niet in staat waren om de prostaatkanker PDA te evalueren of een gecontroleerde studie uit te voeren van SDM implementatie bij ES-SCLC patiënten omwille van de kleine patiëntenpopulatie. Desalniettemin geven onze bevindingen een overzicht van de barrières en facilitatoren voor SDM en PDA implementatie op verschillende niveaus (individueel, organisatorisch, en extern) en strategieën om clinici en patiënten te ondersteunen in hun samenwerking. 


\section{Impact statement}

As patient participation in healthcare decisions continues to grow, there is a greater need for decision support tools and processes, such as patient decision aids (PDAs) and shared decision-making (SDM) respectively, that help patients and clinicians collaborate. These innovations often fail at the implementation stage because of a lack of attention to user perspectives and workflows. Therefore, in this thesis we investigated what matters to patients, how they experience the decision-making process, and how patient-clinician communication can be enhanced. In this section, we present the scientific and societal impact of our findings.

\section{Scientific impact}

\section{Development of a prostate cancer PDA}

The outcome of the first three studies described in Chapters 2-4 of this thesis was a web-based PDA for prostate cancer patients that is publicly available on http:// beslissamen.nl. Since patients considered the consequences of treatment side-effects on their daily lives important, it was later decided to augment the PDA with prediction models that present the probability of an individual patient experiencing a certain side-effect, such as incontinence or erectile dysfunction, based on his clinical characteristics. The implementation of the augmented PDA is underway. As of July 2021, initial work has been conducted to understand how prostate cancer patients evaluate risk information. This work will form the basis for fine-tuning the PDA to display risk predictions in a format that is preferable to patients. Once implemented, we hypothesize that the PDA will increase the level of SDM in the consultation and patient knowledge about prostate cancer and the relevant treatments, and decrease the level of decisional conflict. These variables will be measured by means of the validated SDM-Q-9 and SDM- 
Q-Doc questionnaires (for patients and clinicians respectively), a 20item knowledge test with true/false questions, and the validated Decisional Conflict Scale questionnaire. In addition, patients' preferred level of participation in decision-making will be measured by means of the Control Preferences Scale. An impact study will be performed that compares these values directly after the decision-making consultation and three months after the consultation with an intervention group that receives the PDA and a control group that undergoes usual care (without the PDA). At a broader level, our current findings illustrate the principles of user-centered design, namely the process of involving end-users early, understanding their needs and requirements, incorporating user feedback and evaluating design prototypes in an iterative and dynamic manner. This process enhances user-friendliness and increases ownership, particularly among clinicians, which make successful PDA uptake more likely.

\section{Implementing SDM in lung cancer}

Within oncology, the interest in promoting SDM has generally been centered around preference-sensitive conditions such as prostate cancer and breast cancer. In these disease areas there are multiple treatment options with different benefits and harms, and there is room for the patient's preferences to be taken into account. In contrast, conditions such as lung cancer have received little attention in the SDM literature. Lung cancer is characterized by rapid progression, severe complications, and serious side-effects from treatment, resulting in a decision-making process that is more dominated by clinical guidelines than other cancer types. These guidelines tend to prioritize progression-free survival and do not sufficiently incorporate the patient perspective, even though lung cancer is considered a terminal illness, and palliative/end-of-life care decisions are preference-sensitive. In Chapters 5 and $\mathbf{6}$ of this thesis we conducted exploratory work on incorporating SDM into lung cancer decision-making through clinical decision support systems 
(CDSSs) and SDM training. Our findings have been published in a peer-reviewed journal and add to the small but growing literature on SDM in lung cancer. Our results provide insights for researchers and CDSS developers on specific lung cancer use cases where prediction models could provide added benefit, and emphasize the importance of clinician involvement in the development process. Encouragingly, we observed a growing awareness among lung cancer specialists regarding the potential benefits of artificial intelligence (AI) to assist decision-making as well as implementation barriers such as lack of trust. Researchers may further explore the notion of clinician trust, such as how clinicians define trust and how AI-based predictions can be made interpretable so that these predictions form a reliable component of data-driven SDM.

\section{Adapting PDA format}

PDAs come in a variety of formats (digital, print, information booklets), but by and large the most common ones are digital. These are easier to update and offer the benefit of having multimedia content. This thesis lays out the challenges and benefits of both types of PDAs. We established that digital PDAs offer a multitude of possibilities to help patients understand their disease and treatment options, clarify their values, and test their knowledge. In-consultation paper PDAs, on the other hand, bring this process into the clinical interaction and provide a structure for the SDM conversation, as shown in Chapter 7. By interviewing clinicians who have used paper PDAs in their consultations, we were able to establish that this format was instrumental in structuring the SDM conversation and helped clinicians put the main steps of the SDM process into action. On the basis of our findings, researchers can further explore ways to integrate digital and paper PDAs so that clinicians and patients can benefit from decision support that is adapted both for consultations as well as home use by patients. An example would be a hybrid PDA with an interactive digital version containing multimedia content for the patient to use at home and a 
corresponding printed version in the form of decision cards, structured according to the SDM steps, that the patient and clinician can use during the consultation.

\section{Societal impact}

\section{Patient-clinician collaboration}

Throughout the course of this thesis, we observed that the majority of patients we enlisted in the PDA development phase as well as those who participated in the PCI decision were enthusiastic about SDM. However, most patients still expect the paternalistic model of decisionmaking and thus taking an equal role in decision-making with the clinician is not yet the norm. The creation of the publicly available PDA on http:/ / beslissamen.nl can spread awareness among newly-diagnosed patients about the fact that multiple treatment options exist with different harms and benefits. With greater knowledge about their options, patients can more readily ask questions and participate in a dialogue with their clinician, even in contexts where SDM has not yet been formally implemented. Furthermore, patient organizations can play a role in bringing SDM to the fore, disseminating PDAs, and helping prepare patients for a SDM consultation in terms of being informed and the type of questions to ask.

By involving clinicians in the development process of the prostate PDA and in SDM training for lung cancer, our work increased clinicians' awareness of the patients' perspectives and desire for participation. Our findings from the Danish context (Vejle Hospital), where SDM/PDA implementation was well underway, showed that empowering clinicians with practical tools like structured in-consultation PDAs to put knowledge into practice is a key success factor. This approach is complementary to Maastro's digital PDAs, and our results provide a first step to developing a hybrid PDA that combines the strengths of both digital and paper PDA formats. This has become particularly relevant in light of the COVID-19 pandemic, 
in which many consultations had to be conducted virtually. Digital PDAs are ideal in such circumstances, and Vejle Hospital is currently exploring ways to digitize the existing paper PDAs so that patients have access to decision support regardless of location. This thesis is the first step in mutual learning between the two institutes and offers new possibilities in promoting patient-clinician collaboration.

\section{Redesigning patient pathways}

Our findings shed light on the current clinical workflow in prostate cancer and lung cancer in the Netherlands and the possible barriers it can introduce to SDM/PDA implementation. Although considerable efforts are expended to support patients in SDM, much progress can be made by studying and modifying the treatment trajectory so that patient preferences are not only considered in the consultation but are honored throughout the patient pathway. On the basis of our findings, the role of GPs in the treatment trajectory can be formalized and their possible involvement in PDA implementation can be increased. The role of nurses can also be expanded as patients perceive them to be more approachable.

\section{Aligning incentives}

A key finding in our work was that the current treatment trajectory can incentivize certain treatments over others due to factors such as volume quotas. An implication of our work is that stakeholders in the current healthcare system, such as health insurers, can play a significant role in aligning these incentives. The lung cancer study described in this thesis was a collaboration between Maastro and the Dutch healthcare insurer CZ. Through this collaboration, named My Best Treatment, SDM was implemented and evaluated in the extensive-stage small-cell lung cancer trajectory. Such collaborations give health insurers insight into the decision-making process and effects of SDM so that reimbursement structures can be redefined to stimulate SDM. 



\section{Acknowledgments}

This thesis was made possible thanks to the feedback, support, and advice of many people, not least the members of the assessment committee who devoted considerable time to read and review my work. Thank you Dr. Trudy van der Weijden, Dr. Maria Jacobs, Dr. Maurits Kaptein, Dr. Daniela Raphael, and Dr. David Townend.

I would like to thank my supervisor, Andre Dekker, for his invaluable support and guidance over the course of my $\mathrm{PhD}$. He gave me many opportunities to not only pursue my research interests but to develop myself as a person. Andre, you are the true definition of a leader and I feel incredibly lucky to have been a part of your research group.

My co-promoters, Rianne Fijten and Cheryl Roumen, were a constant source of encouragement throughout the ups and downs that characterize a PhD. They cheered me on in the good times and motivated me to keep going when I felt stuck. Rianne, your 'can do' spirit made me feel like anything is possible. Your systematic approach balanced with an openness to discussion made working with you a great learning experience. Cheryl, you always make time for people and go out of your way to help. I truly appreciate all the help you gave me. Thank you!

Many thanks also to Adriana Berlanga with whom I began my $\mathrm{PhD}$ journey at MAASTRO. Even though we worked together for a short time, your advice and presence gave me a lot of reassurance as I took my first steps in clinical research, which was a new area for me.

I would also like to thank my Danish colleagues at Vejle Hospital and the Center for Shared Decision-Making, starting with Dr. Karina Dahl Steffensen. Karina, my time in Vejle was one of the highlights of my $\mathrm{PhD}$ journey and I'm deeply grateful to you for making my visit possible. Aside from the valuable time spent in the clinic, I have fond memories of our walk together near your home and meeting your family over home-made pizza. Many thanks to the entire team at the Center: Karina Olling, Rikke Madsen, Lisbeth Gamst, Charlotte Hald Fausbøll, 
Margit Søgaard, Stine Rauff Søndergaard, Karina Mølgaard Jensen, Anette Kargo, and especially Sarah Ditte Andersen, for welcoming me and helping to make my visit enjoyable and fruitful.

I am very grateful to all the clinicians, nurses, and patients who participated in our research studies. Our work together was supported by the efforts of MAASTRO's Clinical Trial Office and the software development team, in particular: Sylvie Canisius, Ruud Houben, Claudia Offermann, Rody Zuidema, and Peter Fick.

A big thank you to the amazing high-vlaaiers I got to know through the Clinical Data Science group at MAASTRO: Alberto, Ananya, Audrey, Biche, Chong, Dennis, Ibrahim, Inigo, Ivan, Jasper, Johan, Junhua, Karen, Leonard, Nikhil, Sander, Sonia and Sonia, Stuti, Suraj, and Zhenwei. Special thanks to Hajar, Matthijs and Petros, my fellow scrum mates. Chatting with the three of you every morning made my day begin on a great note :)

I would like to acknowledge Amrapali Zaveri, who, although no longer with us, continues to be an inspiration. Amrapali, you were not only a brilliant scientist but a dear friend whose warmth and sense of humour put a smile on the face of all who were lucky enough to know you. You have no idea how much you helped me.

My work would not have been possible without the tremendous love and support of my family. My dear sister, Anupriya, thank you for being my sounding board and always listening to my ranting and raving. Hanging out with you and Kedar always recharges my batteries. Siddhant and Keshav, your curiosity, energy, and excitement remind us all of what is important in life. Most of all, I would like to thank my parents. Anything I have achieved in my life is due to their unconditional love and unwavering belief in me. I am eternally grateful to you all. This thesis was fuelled by masala chai.

Anshu Ankolekar

Maastricht

October 29, 2021 


\section{Published work}

\section{Publications}

1. Ankolekar, A., Steffensen, K.D., Olling, K., Dekker, A., Wee, L., Roumen, C., Hasannejadasl, H., \& Fijten, R. (2021). Practitioners' views on shared decision-making implementation: A qualitative study. PLOS One (forthcoming)

2. Ankolekar, A., De Ruysscher, D., Reymen, B., Houben, R., Dekker, A., Roumen, C., \& Fijten, R. (2021). Shared decision-making for prophylactic cranial irradiation in extensive-stage small-cell lung cancer: an exploratory study. Translational Lung Cancer Research, 10(7), 3120.

3. Kazmierska, J., Hope, A., Spezi, E., Beddar, S., Nailon, W. H., Osong, B., Ankolekar, A., Choudhury, A., Dekker, A., Redalen, K.R. \& Traverso, A. (2020). From multisource data to clinical decision aids in radiation oncology: the need for a clinical data science community. Radiotherapy and Oncology.

4. Ankolekar, A., Vanneste, B. G., Bloemen-van Gurp, E., Van Roermund, J. G., van Limbergen, E. J., Van de Beek, K., Marcelissen, T., Zambon, V., Oelke, M., Dekker, A., Roumen, C., Lambin, P., Berlanga, A., \& Fijten, R. (2019). Development and validation of a patient decision aid for prostate cancer therapy: from paternalistic towards participative shared decision making. BMC medical informatics and decision making, 19(1), 1-11.

5. Ankolekar, A., Dekker, A., Fijten, R., \& Berlanga, A. (2018). The benefits and challenges of using patient decision aids to support shared decision making in health care. JCO clinical cancer informatics, 2, 1-10. 


\section{Submitted}

(* denotes equal contribution)

1. Ankolekar, A.*, Van Der Heijden, B.*, Dekker, A., Roumen, C., De Ruyss- cher, D., Reymen, B., Berlanga, A., Oberije, C., \& Fijten, R. (2021). Clinician Perspectives on Clinical Decision Support Systems in Lung Cancer: Implications for Shared DecisionMaking. (submitted, Health Expectations)

2. Ankolekar, A., Vanneste, B., Bloemen-van Gurp, E., Van Roermund, J., Berlanga, A., Roumen, C., Van Limbergen, E., Lutgens, L., Marcelissen, T., Lambin, P., Dekker, A., \& Fijten, R. (2021). Implementing Shared Decision-Making in Prostate Cancer: Insights from Primary and Secondary Care (Submitted, ZEFQ)

\section{Conference abstracts/presentations}

1. Shared Decision-Making and Decision Support for Prophylactic Cranial Irradiation in SCLC Patients. ESTRO 39 (2020), virtual conference. (Poster presentation)

2. Implications of Clinicians' Attitudes towards Clinical Decision Support Systems. MIE (2020), Geneva, Switzerland. (poster)

3. Shared Decision-Making and Decision Support for Prophylactic Cranial Irradiation in SCLC Patients. 3rd ESTRO Physics Workshop (2019), Budapest, Hungary (Presentation)

4. Barriers and Potential Solutions Regarding the Implementation of Patient Decision Aids. 10th International Shared Decision Making Conference (2019), Quebec City, Canada (Presentation)

5. Development and Validation of a Prostate Cancer Patient Decision Aid: Towards Participative Medicine. ESTRO 38 (2019), Milan, Italy (Poster presentation) 
6. Defining an effective implementation strategy for shared decision-making: a multi-faceted approach. 9th International Shared Decision Making Conference (2017), Lyon, France (Poster) 



\section{About the author}

Anshu Ankolekar was born on 10 December, 1983 in Mumbai, India. Upon finishing her secondary education at Jeanne d'Arc College in Maastricht, she studied International Business at Maastricht University, graduating in 2007. She worked as a research assistant in behavioral finance before pursuing further studies in Econometrics and Operations Research at Tilburg University, culminating in a Master's degree in Finance in 2013. Her thesis explored innovation in the pharmaceutical industry. Following her graduation, she worked in clinical trial software development at Cytel.

In 2016, Anshu joined a MAASTRO project to develop and implement a patient decision aid for prostate cancer patients. She became a PhD candidate in 2017 when she joined the GROW School for Oncology and Developmental Biology at the Faculty of Health, Medicine and Life Sciences (Maastricht University). As part of her research, she helped develop initiatives to implement shared decision-making and improve patient care in various disease areas (such as prostate, lung, and brain cancer) and a general patient decision aid for clinical trial participation. Between February and March 2020, she visited the Center for Shared Decision-Making at Vejle Hospital in Denmark, under the guidance of Dr. Karina Dahl Steffensen and funded by a technology transfer grant from the European Society for Radiotherapy \& Oncology (ESTRO). Here, she had the opportunity to study shared decision-making implementation in a different cultural context and share knowledge between the two institutes. As the scientific evidence base and accessibility to information continue to grow, her interest is in science communication to bridge the gap between research and the public. 